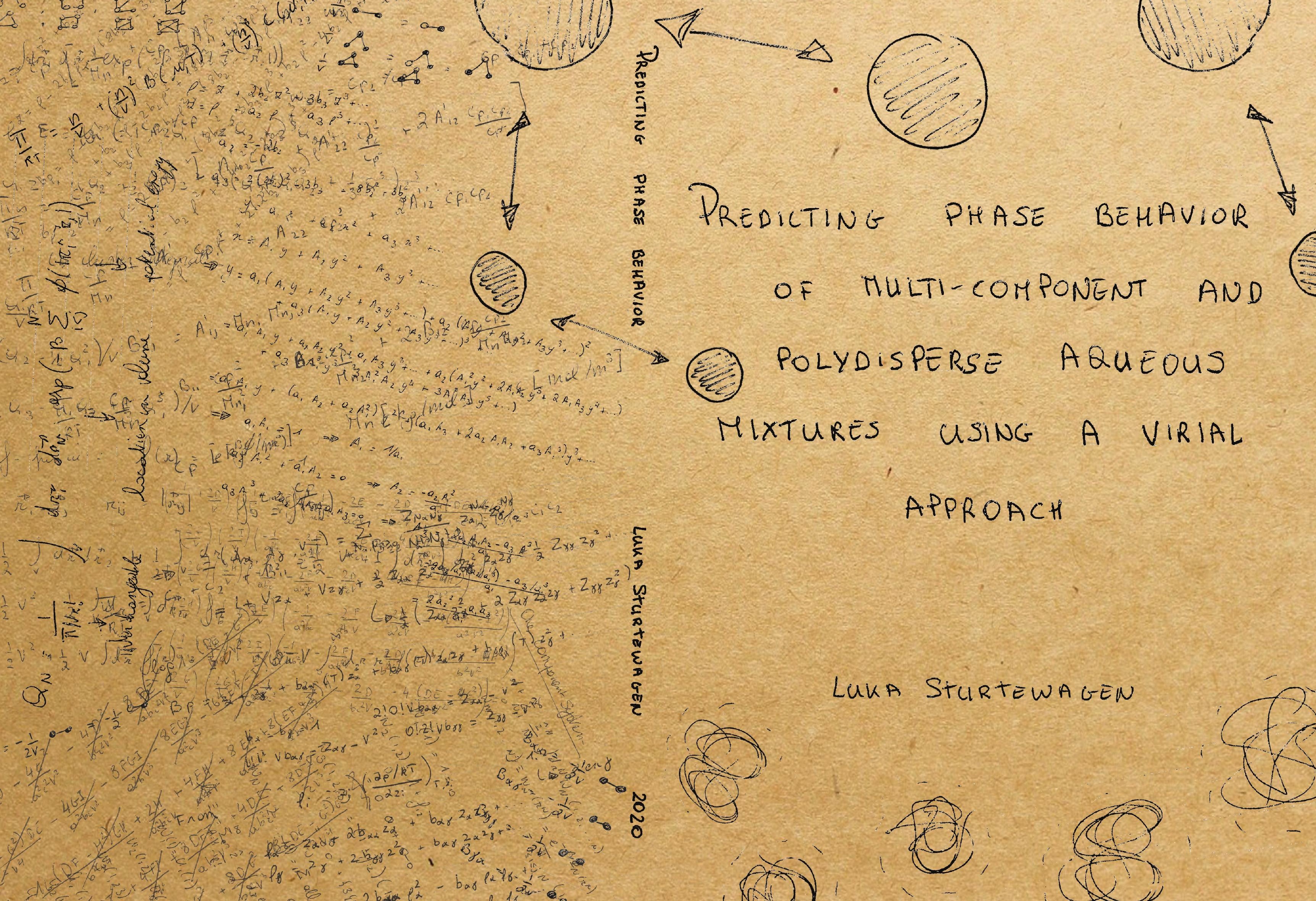




\section{Predicting phase behavior of multi-component and polydisperse aqueous mixtures using a virial approach}

Luka Sturtewagen 


\section{Thesis committee}

\section{Promotor}

Prof. dr. E. van der Linden

Professor of Physics and Physical Chemistry of Foods

Wageningen University \& Research

\section{Copromotor}

Dr. H.G.J. van Mil

Lecturer at the Mathematical Institute

Leiden University

\section{Other members}

Prof. dr. B. Bergenståhl, Lund University, Sweden

Prof. dr. P. Fisher, ETH Zürich, Switzerland

Prof. dr. J. Molenaar, Wageningen University \& Research

Prof. dr. B.M. Mulder, Institute for Atomic and Molecular Physics, Amsterdam

This research was conducted under the auspices of the Graduate School VLAG (Advanced studies in Food Technology, Agrobiotechnology, Nutrition and Health Sciences) 


\title{
Predicting phase behavior of multi-component and polydisperse aqueous mixtures using a virial approach
}

\author{
Luka Sturtewagen
}

Thesis

submitted in fulfillment of the requirements for the degree of doctor at Wageningen University by the authority of the Rector Magnificus,

Prof. dr. A.P.J. Mol,

in the presence of the

Thesis Committee appointed by the Academic Board

to be defended in public

on Wednesday, $11^{\text {th }}$ of November 2020

at 1.30 p.m. in the Aula. 
Luka Sturtewagen

Predicting phase behavior of multi-component and polydisperse aqueous mixtures using a virial approach,

210 pages.

PhD thesis, Wageningen University, Wageningen, the Netherlands (2020)

With references, with summary in English

ISBN 978-94-6395-377-1

DOI $10.18174 / 519721$ 


\section{Contents}

List of Figures iii

List of Tables vii

List of Symbols $\quad$ ix

1 General introduction $\quad 1$

2 Effect of size polydispersity $\quad 15$

3 Effect of non-additivity

$\begin{array}{lll}4 & \text { Ternary mixtures of hard spheres } & 99\end{array}$

$\begin{array}{lll}5 & \text { Polydisperse colloids } & 147\end{array}$

$\begin{array}{llr}6 & \text { General discussion } & 179\end{array}$

$\begin{array}{ll}\text { Summary } & 197\end{array}$

$\begin{array}{lr}\text { Acknowledgments } & 201\end{array}$

$\begin{array}{ll}\text { About the author } & 205\end{array}$ 



\section{List of Figures}

1.1 Schematic depection of depletion interaction.

1.2 Schematic depection of the phase diagram.

2.1 Graphical representation of a simple polydisperse mixture. 30

2.2 Phase diagram for monodisperse binary mixture. 41

$2.3 \quad$ Phase diagram for binary polydisperse mixture $(P D=4.00) . \quad 42$

2.4 Phase diagram for binary polydisperse mixture $(P D=8.00) . \quad 43$

2.5 Phase diagram for binary polydisperse mixture (skewed distribution). 44

2.6 Phase diagram for binary polydisperse mixture, full distribution. 46

3.1 Graphical representation of a simple polydisperse mixture. 61

3.2 Phase diagram for binary non-additive polydisperse mixture. 66

3.3 Phase diagram for binary non-additive sub-components $(P D=4.00) . \quad 67$

3.4 Phase diagram for binary non-additive sub-components $(P D=8.00 / 12.00) . \quad 68$

3.5 Phase diagram for binary non-additive sub-components (skewed). 70

3.6 Phase diagram for binary non-additive sub-components (skewed). 71

3.7 Phase diagram for binary mixture $\left(q=1 / 3, \Delta_{A B}=0.075, P D=4\right) . \quad 73$

3.A.1 Phase diagram for binary non-additive polydisperse mixture $(P D=8 / 12) . \quad 81$

3.A.2 Phase diagram for binary non-additive polydisperse mixture (skewed). 82

3.A.3 Phase diagram for binary non-additive polydisperse mixture (skewed). 83

3.A.4 Phase diagram for binary mixture $\left(q=1 / 3, \Delta_{A B}=0.05, P D=4\right)$. 84

3.A.5 Phase diagram for binary mixture $\left(q=1 / 3, \Delta_{A B}=0.075, P D=4\right) . \quad 85$

3.A.6 Phase diagram for binary mixture $\left(q=1 / 3, \Delta_{A B}=0.1, P D=4\right) . \quad 86$

3.A.7 Phase diagram for binary mixture $\left(q=1 / 3, \Delta_{A B}=0.05, P D=8\right) . \quad 87$

3.A.8 Phase diagram for binary mixture $\left(q=1 / 3, \Delta_{A B}=0.075, P D=8\right) . \quad 88$

3.A.9 Phase diagram for binary mixture $\left(q=1 / 3, \Delta_{A B}=0.10, P D=8\right) . \quad 89$

3.A.10 Phase diagram for binary mixture $\left(q=1 / 3, \Delta_{A B}=0.05, P D=12\right) . \quad 90$

3.A.11 Phase diagram for binary mixture $\left(q=1 / 3, \Delta_{A B}=0.075, P D=12\right) . \quad 91$

3.A.12 Phase diagram for binary mixture $\left(q=1 / 3, \Delta_{A B}=0.10, P D=12\right) . \quad 92$ 
4.1 Graphical representation of a simple ternary mixture. 105

4.2 Phase diargram for binary $A B$ mixture. 110

4.3 Phase diagram of ternary mixture $A B C\left(\Delta_{A C}=-0.1\right.$, and $\left.\Delta_{B C}=-0.1\right) . \quad 112$

4.4 Phase diagram of ternary mixture $A B C\left(\Delta_{A C}=-0.1\right.$, and $\left.\Delta_{B C}=0\right) . \quad 113$

4.5 Phase diagram of ternary mixture $A B C\left(\Delta_{A C}=0\right.$, and $\left.\Delta_{B C}=-0.1\right) . \quad 115$

4.6 Phase diagram of ternary mixture $A B C\left(\Delta_{A C}=0\right.$, and $\left.\Delta_{B C}=0\right) . \quad 116$

4.7 Phase diagram of ternary mixture $A B C\left(\Delta_{A C}=0.1\right.$, and $\left.\Delta_{B C}=-0.1\right) . \quad 117$

4.8 Phase diagram of ternary mixture $A B C\left(\Delta_{A C}=0.1\right.$, and $\left.\Delta_{B C}=0\right) . \quad 119$

4.9 Phase diagram of ternary mixture $A B C\left(\Delta_{A C}=-0.1\right.$, and $\left.\Delta_{B C}=0.1\right) . \quad 120$

4.10 Phase diagram of ternary mixture $A B C\left(\Delta_{A C}=0\right.$, and $\left.\Delta_{B C}=0.1\right) . \quad 121$

4.11 Phase diagram of ternary mixture $A B C\left(\Delta_{A C}=0.1\right.$, and $\left.\Delta_{B C}=0.1\right) \quad 122$

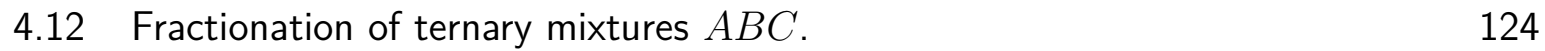

4.13 Fractionation of ternary mixtures $A B C$. 125

4.14 Phase diagram of ternary mixture $A B C\left(q_{A C}=1 / 5\right) . \quad 127$

4.15 Phase diagram of ternary mixture $A B C\left(\Delta_{A C}=0.15\right.$, and $\left.\Delta_{B C}=0.1\right) . \quad 129$

4.B.1 Fractionation of ternary mixture $A B C\left(\Delta_{A C}=-0.1\right.$, and $\left.\Delta_{B C}=-0.1\right) . \quad 138$

4.B.2 Fractionation of ternary mixture $A B C\left(\Delta_{A C}=-0.1\right.$, and $\left.\Delta_{B C}=0\right) . \quad 139$

4.B.3 Fractionation of ternary mixture $A B C\left(\Delta_{A C}=0\right.$, and $\left.\Delta_{B C}=-0.1\right) . \quad 140$

4.B.4 Fractionation of ternary mixture $A B C\left(\Delta_{A C}=0\right.$, and $\left.\Delta_{B C}=0\right)$. 141

4.B.5 Fractionation of ternary mixture $A B C\left(\Delta_{A C}=0.1\right.$, and $\left.\Delta_{B C}=-0.1\right) . \quad 142$

4.B.6 Fractionation of ternary mixture $A B C\left(\Delta_{A C}=0.1\right.$, and $\left.\Delta_{B C}=0\right) . \quad 143$

4.B.7 Fractionation of ternary mixture $A B C\left(\Delta_{A C}=-0.1\right.$, and $\left.\Delta_{B C}=0.1\right) . \quad 144$

4.B.8 Fractionation of ternary mixture $A B C\left(\Delta_{A C}=0\right.$, and $\left.\Delta_{B C}=0.1\right) . \quad 145$

4.B.9 Fractionation of ternary mixture $A B C\left(\Delta_{A C}=0.1\right.$, and $\left.\Delta_{B C}=0.1\right) . \quad 146$

5.1 Graphical representation of binary mixture of colloids. 153

5.2 Size distribution of PEO and Dextran. 158

5.3 Phase diagram for polydisperse PEO $(N=2)$ and dextran $(N=1) . \quad 159$

5.4 Phase diagram for polydisperse PEO $(N=5)$ and dextran $(N=1) . \quad 160$

5.5 Phase diagram for polydisperse PEO $(\mathrm{N}=1)$ and dextran $(\mathrm{N}=2) . \quad 161$

5.6 Phase diagram for polydisperse PEO $(\mathrm{N}=1)$ and dextran $(\mathrm{N}=10) . \quad 162$

5.7 Phase diagram for polydisperse PEO $(\mathrm{N}=2)$ and dextran $(\mathrm{N}=2)$.

5.8 Phase diagram for polydisperse PEO $(N=5)$ and dextran $(N=10) . \quad 165$

5.A.1 Molar weight distribution from SEC for dextran. 178

5.A.2 Molar weight distribution from SEC for polyethylene glycol. 178

$\begin{array}{lll}6.1 & \text { Schematic overview of thesis. } & 180\end{array}$ 
6.2 Total volume fraction at critical point HS monodisperse binary mixtures. 182

6.3 Total volume fraction at critical point depending on polydispersity. 183

6.4 Total volume fraction at critical point depending on polydispersity. 184

6.5 Non-additive Hard sphere interaction. 186

6.6 Total volume fraction at critical point of NAHS binary mixtures. 187

$\begin{array}{lll}6.7 & \text { Theoretical phase diagram of a three phase system. } & 191\end{array}$

6.8 Three phase system of gelatin, dextran, and PEO. 192 



\section{List of Tables}

2.1 Critical points and fractionation binary polydisperse mixtures.

2.2 Distribution of polydisperse component in demixed phases.

3.1 Critical points and fractionation binary NAHS polydiseprse mixture.

3.2 Distribution of polydisperse component in demixed phases, NAHS. 76

3.B.1 Critical points and fractionation binary sub-components $P D=8$, NAHS. 93

3.B.2 Critical points and fractionation binary sub-components $P D=12$, NAHS. $\quad 94$

3.B.3 Critical points and fractionation binary sub-components, skewed, NAHS. 95

3.B.4 Critical points and fractionation binary sub-components, skewed, NAHS. 96

3.B.5 Critical points and fractionation binary sub-components, skewed, NAHS. $\quad 97$

3.B.6 Critical points and fractionation binary sub-components, skewed, NAHS. 98

4.1 Virial coefficient ratios ternary mixtures. 123

4.2 Virial coefficient ratios three phase systems. 128

4.A.1 Fractionation ternary $A B C$ mixture $(\eta(0.05,0.20,0.10))$. 134

4.A.2 Fractionation ternary $A B C$ mixture $(\eta(0.05,0.20,0.25))$. 135

4.A.3 Fractionation ternary $A B C$ mixture $(\eta(0.10,0.20,0.10))$. 136

4.A.4 Fractionation ternary $A B C$ mixture $(\eta(0.05,0.10,0.10)) . \quad 137$

5.1 Virial coefficients and critical point of PEO35 and D100. 156

5.2 Distribution of PEO $(N=5)$ and dextran $(N=1)$ in demixed phases. 167

5.3 Distribution of PEO $(N=1)$ and dextran $(N=10)$ in demixed phases. 169

5.4 Distribution of PEO $(N=5)$ and dextran $(N=10)$ in demixed phases. 171 



\section{List of Symbols}

$\alpha \quad$ Volume fraction phase $I \alpha=\frac{V^{I}}{V^{I}+V^{I I}}$

$\beta \quad \frac{1}{k T}$

$\Delta \quad$ Non-additivity parameter for interacting particles

$\eta \quad$ Packing fraction $\frac{\pi \rho \sigma^{3}}{6}$

$\Lambda \quad$ The thermal wavelegnth $\left(\frac{h^{2}}{2 \pi m k T}\right)^{1 / 2}$

$\mu_{i} \quad$ Chemical potential of component $i$

$\mu_{s} \quad$ Chemical potential of the solution

$\nu_{i} \quad$ Compound of type $\nu_{i}$

$\phi_{i j} \quad$ Pair potential between particle $i$ and $j$

П Osmotic pressure

$\rho \quad$ Density $\frac{N}{V}$

$\sigma \quad$ Diameter of a particle

$\vec{p}_{i} \quad$ Impulse of the particle

$\vec{r}_{i} \quad$ Position of the particle

$\Xi \quad$ Grand canonical partition function

A Helmholtz free energy

$B \quad$ Virial coefficient

$B_{2} \quad$ Second virial coefficient

$b_{l} \quad$ Clusterintegral of $l$ particles

$f \quad$ Number of coexisting phases

$f_{12} \quad$ Mayer $\mathrm{f}$-function for particle 1 and 2

$H \quad$ Hamiltonian

$h \quad$ Planck's constant

$K \quad$ Kinetic energy

$k \quad$ Boltzmann's constant

$M_{1} \quad$ Stability matrix 
$M_{2} \quad$ Partial derivative matrix of Stability matrix

$m_{s} \quad$ Mass of the solvent particle

$m_{\nu} \quad$ Mass of the solute particle

$N \quad$ Number of particles

$n \quad$ Number of distinguishable components

$N_{s} \quad$ Number of solvent particles

$N_{\nu} \quad$ Number of solute particle

$p \quad$ Pressure

$p^{i} \quad$ Momentum of particle $i$

$P D \quad$ Degree in polydispersity

$Q \quad$ Configuration integral

$q \quad$ Size ratio between components $\frac{\sigma_{A}}{\sigma_{B}}$

$q^{i} \quad$ Velocity of particle $i$

$q_{i j} \quad$ Size ratio between components $\frac{\sigma_{i}}{\sigma_{j}}$

$S \quad$ Entropy

$T \quad$ Temperature

$U \quad$ Potential energy

$V \quad$ Volume

$W(r)$ Interaction potential

$W_{\nu} \quad$ Pair potential for disolved compounds

$x \quad$ Particle of type $x$

$y \quad$ Particle of type $y$

$Z \quad$ Canonical partition function

$z \quad$ Activity $\frac{e^{\beta \mu}}{\Lambda^{3}}$ 


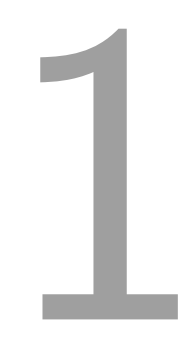

General introduction 


\subsection{Introduction}

Aqueous mixtures consist generally of many components. Depending on the type of components, their interactions, and their concentrations these mixtures form a stable, homogeneous mixture or they are unstable and demix into two or more liquid phases. In the study of phase behavior of binary mixtures, these mixtures are often simplified and assumed to be mixtures of two pure monodisperse components. Often, however these mixtures contain components that exhibit varying degrees of polydispersity in terms of their size, shape, and charge, complicating the prediction of the phase behavior of a mixture. Even small impurities can lead to drastic changes in the position of the phase boundary (Sager, 1998). Besides that the compatibility between components can be dependent on the temperature (Edelman et al., 2001), salt concentration or pH of the solution (Kontogiorgos et al., 2009). The investigation of mixtures with more than two components has a considerable amount of challenges as with an increasing amount of considered components, the number of interactions that have to be taken into account changes drastically. Next to that, with an increasing number of components, the number of theoretical phases that can be formed increases as well. According to the Gibbs phase rule, which gives the relationship between the degrees of freedom ( $d o f$ ) of a system, such as the mole fraction of the components, the temperature, or the pressure, and the maximum number of theoretical phases $(f)$ of a system, the number of possible phases increases with the number of components $(n)$. The degrees of freedom of a system is given by $d o f=n+2-f$.

In this thesis we aimed to get a better understanding into the phase behavior of mixtures with more than two components in solution. We will focus on the liquid-liquid demixing. When looking at multicomponent systems, one may distinguish chemically equivalent components that only differ in size (polydisperse components) and chemically different components. Both types of mixtures will be investigated. We note that practically relevant components are often naturally polydisperse e.g. colloids such as dextran (Edelman et al., 2001) or contain significant amounts of impurities e.g. plant protein extracts (Pojić et al., 2018).

\subsection{Phase behavior of a mixture}

Dilute aqueous mixtures generally form homogeneous solutions. At higher concentrations, depending on the interactions between the components and their respective concentrations the mixture may become unstable and demix. Examples of mixtures that show phase separation are aqueous mixtures of agar and starch or gelatin, systems first studied by 
Beijerinck in 1896 (Aichinger et al., 2017) and since then many others have been found. Phase separation is generally driven by either complex coacervation or segregative phase separation (Turgeon et al., 2003). In the case of complex coacervation, demixing is due to association between components (van de Velde et al., 2015). In segregative phase separation, the driving force is the depletion force induced by different excluded volumes (De Bont et al., 2002; Tuinier et al., 2003; Lekkerkerker and Tuinier, 2011). The depletion force is a net attraction. Each of the particles is surrounded by a layer in which the centers of another particle cannot enter, i.e. depleted (Figure 1.1). When two particles of the same species approach each other, their depletion layers overlap, resulting in an imbalanced osmotic pressure. This prompts an increase in available volume for the other particles, leading to an increase in total entropy and a decrease in free energy (De Bont et al., 2002).

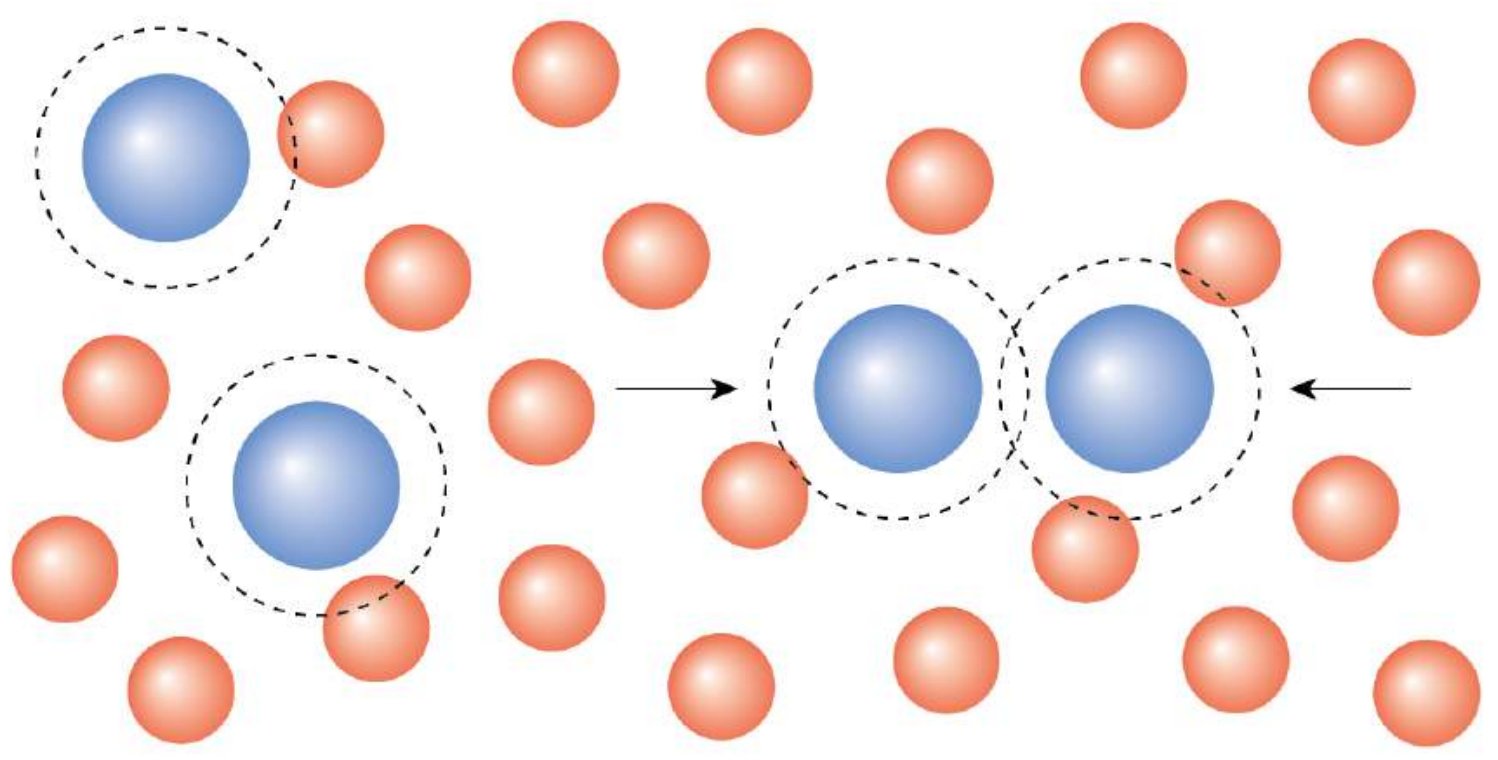

Figure 1.1: Schematic depiction of depletion layers of hard spheres. The depletion layers, in this case, the volume around the large spheres that is inaccessible inaccessible to the centers of the small spheres, are indicated by the dashed lines. When there is overlap of the depletion layers, the small spheres are depleted from the overlapping region, resulting in a net attraction due to a reduction of osmotic pressure in the overlapping region. The net attraction is indicated by the arrows (Lekkerkerker and Tuinier, 2011).

In this thesis we will focus on segregative phase separation. The interaction between particles are expressed by the second virial coefficient (McMillan and Mayer, 1945). The second virial coefficient can be used in the prediction of the phase behavior of mixtures and can experimentally be measured by osmometry (Ersch et al., 2016b). In theoretical systems, the particles can be modeled by hard spheres. When the phase separation is solely driven by the excluded volume interactions between the two particles, the depletion force is determined by the sum of their respective radii (Biben and Hansen, 1997). In this 
case a certain asymmetry in the sizes of the particles in the mixture is required for phase separation (Biben and Hansen, 1991). This size difference leads to the depletion of small spheres around the larger spheres and results in an attractive interaction between the larger particles (Dijkstra et al., 1999). This is referred to as additive hard sphere (HS) model. Another model is when the minimum distance between the particles is not the sum of their respective radii. This distance can be larger or smaller. This is referred to as non-additive hard sphere (NAHS) model. Already at small degrees of non-additivity it becomes possible for components with no size asymmetry to demix (Hopkins and Schmidt, 2010; Dijkstra, 1998). When the distance between the spheres becomes smaller than the sum of their respective radii, the excluded volume is smaller. This decreases the repulsive force between the particles of different species, resulting in a mixture that is more stable (Roth et al., 2001). When the distance between the particles becomes larger than the sum of their respective radii, phase separation becomes easier. In either case, upon phase separation, the mixture will demix into two (or more) phases, each enriched in one of the components. All phases are in thermodynamic equilibrium, meaning that the osmotic pressure of all phases is the same, just as the chemical potential of each component is the same over all phases (Hill, 1986).

Figure 1.2 depicts the phase diagram for a monodisperse mixture of two components in solution. The binodal is the phase boundary, concentrations lower than this boundary result in a stable one phase system. Above this boundary, mixtures demix into two phases. When a parent mixture demixes into two phases due to depletion interaction, each phase is enriched in one component, while being depleted in the other. These two phases are connected to each other by a tie line. The length of each tie line segment (the connection between the concentration of the parent mixture and the concentrations of each phase) is a measure for the volume of each of the phases. Demixing into two phases generally happens through two mechanisms: spinodal decomposition or nucleation and growth (Verhaegh et al., 1996). Concentrations above the spinodal demix through spinodal decomposition: in these thermodynamically unstable mixtures long range density variations form, leading first to a bi-continuous network. Between the spinodal and the binodal, mixtures are metastable. In these mixtures demixing happens through nucleation and growth, resulting in isolated droplets. The spinodal and binodal meet each other at the critical point. This is the concentration at which two phases in equilibrium become indistinguishable (Heidemann, 1994).

We chose to utilize a virial approach up to the second term to describe the phase behavior of polydisperse and multi-component mixtures. This choice has been instigated by the consideration that the approach up to the second virial coefficient will systematically build on the results of earlier experimental work by Dewi et al. (2020) and Ersch et al. (2016a,b), 


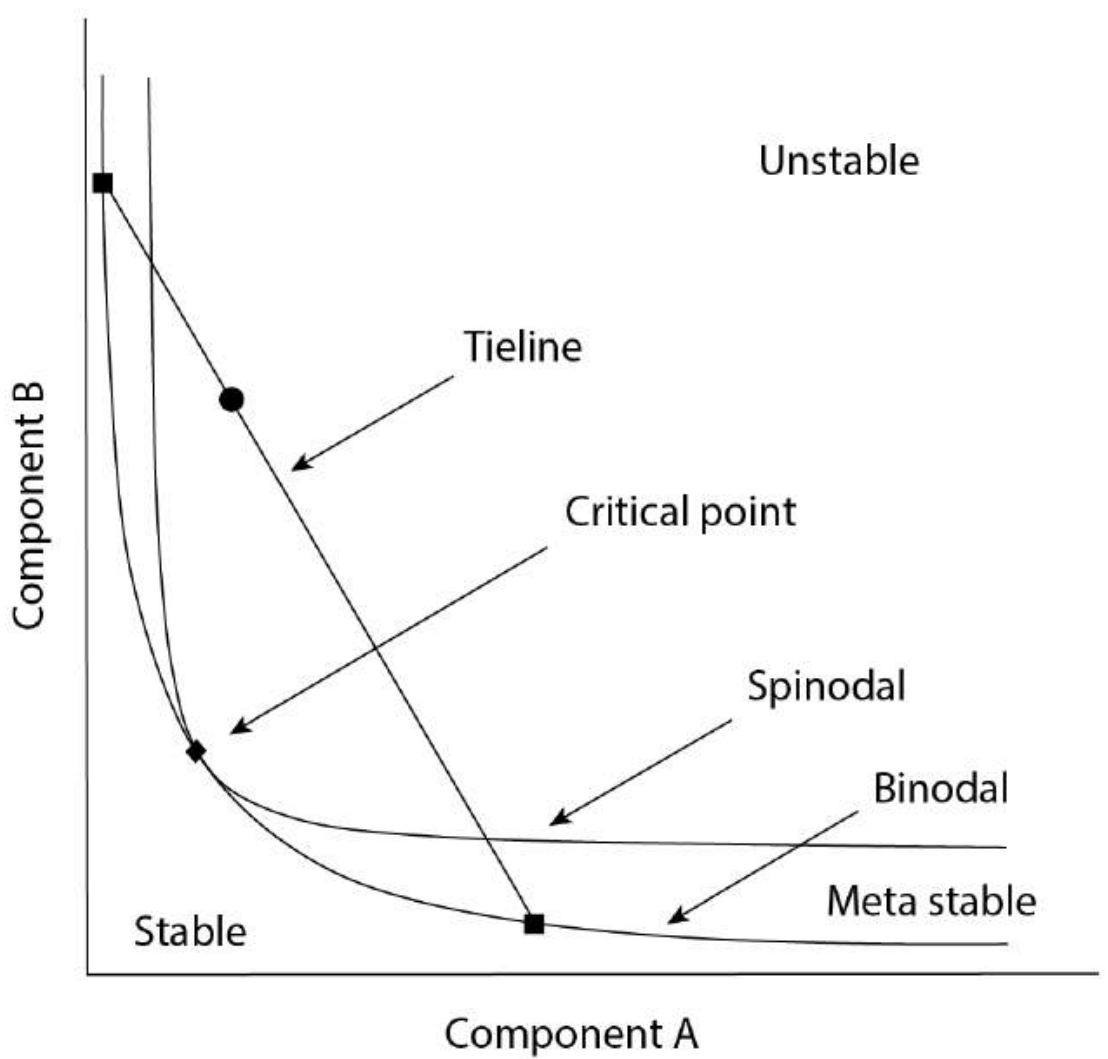

Figure 1.2: Schematic depiction of the phase diagram of a monodisperse binary mixture, the diamond depicts the critical point, the circle depicts a parent mixture that demixes into two phases, indicated by the squares.

and by the consideration that the experimental work published earlier provides support for the relevancy of this approach up to the second order virial coefficient. Furthermore the approach allows for predicting the fractionation of each component in the different phases as a function of the polydispersity of its components.

In Dewi et al. (2020), an experimental phase diagram is depicted for poly-ethylene glycol (PEO35) and Dextran D100. Note, this phase diagram is re-analyzed in Chapter 5 regarding effects of polydispersity of its components. An experimental critical point is located at $(\mathrm{PEO} 35, \mathrm{D} 100)=(0.4,1.0)\left(\mathrm{mol} / \mathrm{m}^{3}\right)$. The concentrations are well below and around the overlap concentration, $c^{*}$, for PEO35 and D100, respectively. This experimental critical point and the values of the viral coefficients up to second order as determined from osmometry (from which a model value for the critical point can be derived) yield a consistent picture. The reduced osmotic pressure follows a straight line with concentrations of PEO35 up to $60 \mathrm{~kg} / \mathrm{m}^{3}$, i.e. up to $3 c^{*}$. For D100 measurements were only done for a reduced osmotic pressure versus concentration up to $0.3 c^{*}$, which also yielded a straight line. Ersch $(2016 a, b)$ investigated the phase behavior of dextran D100 combined with gelatin A and 
finds an experimental critical point located at $(D 100$, Gelatin $A)=(48,25) \mathrm{kg} / \mathrm{m}^{3}$. This critical point and the values of the viral coefficients up to second order as determined from osmometry yield also a consistent picture: the concentrations for the respective individual polymers are much less than $c^{*}$ and the experimental reduced osmotic pressure is linear to at least up to a total polymer concentration less than the $c^{*}$ of either one of the polymers.

\subsection{Polydispersity}

Polydisperse mixtures consist effectively of a large number of sub-components, each with a different size and possibly also different shape or charge (Sollich, 2002). Size polydispersity has an effect on the depletion interaction between all these sub-components. The repulsive barrier between components decreases between polydisperse components (Goulding and Hansen, 2001) and leads to secondary potential energy minima (Walz, 1996; Piech and Walz, 2000; Chu et al., 1996), resulting in a wide range of depletion attractions (Santos et al., 2010) that cannot be treated as homogeneous depletants anymore (Park and Conrad, 2017). This effect on the depletion interaction can, in the short term, stabilize the particle suspension, but destabilize it over time (Tuinier and Petukhov, 2002) and can be further enhanced by the presence of charge variations (Piech and Walz, 2000).

Polydisperse mixtures have theoretically been modeled using both continuous and semicontinuous distributions (Cotterman et al., 1985; Cotterman and Prausnitz, 1985), van der Waals approximations (Bellier-Castella et al., 2000), models based on the Asakura-Oosawa model (Fasolo and Sollich, 2005; Sear and Frenkel, 1997), a universal quasi-chemical model (Kang and Sandler, 1988), and Monte Carlo simulations (Paricaud, 2008; Stapleton et al., 1990).

Polydispersity is found to result in significant fractionation, both experimentally (Edelman et al., 2003; Zhao et al., 2016; Gaube et al., 1993) as well as theoretically (Fasolo and Sollich, 2005; Kang and Sandler, 1988; Stapleton et al., 1990). Bellier-Castella et al. (2000) even predicts the onset of a three phase co-existence at higher polydispersity. Next to that, polydispersity leads to an increase in the extent of the fluid-fluid co-existence (Sear and Frenkel, 1997; Kang and Sandler, 1988) and may hinder gelation in mixtures with a gelling agent (Santos et al., 2010). The experiments of Larsson and Mattiasson (1988) show that for polydisperse mixtures, the phase boundary bends towards the concentration axis of one of the components. 


\subsection{Multi component systems}

When the components differ even more in size and chemical make-up, the depletion interactions become more complicated ( $\mathrm{Ji}$ and Walz, 2013), the pair-potential between particles in multi-component systems can have an additional attractive energy-well or an extended repulsive barrier, both influencing the stability of the dispersion (Park and Conrad, 2017).

One of the difficulties in the theoretical study of multicomponent mixtures is the number of interactions in the system. The total number of pairwise interactions for a mixtures with $n$ components increases according to $\frac{n(n+1)}{2}$. This number increases even more when higher order interactions are taken into account. Mixtures with many components have theoretically been studied by combining the random matrix theory and second virial theory, by filling in the interaction matrix randomly from a selected distribution (Sear and Cuesta, 2003). Depending on the chosen distribution, instabilities predicted using this model may lead to complex coacervation (e.g. when negative virial coefficients are allowed according to the distribution) or segregative phase separation. In this thesis we will also model the interactions for multi-component system. In this case the interactions will be based on non-additive hard sphere interactions (Chapter 4).

Also the experimental work on mixtures with more than two components has difficulties, as the number of samples needed to prepare to get an insight into the phase behavior at different concentrations increases significantly with the number of components in the mixture. Experimental work on mixtures with more than two components that demix into multiple phases have therefore focused on only a few concentrations. An example of a system that demixes into three phases is a system containing the high-molecular colloids dextran, ficoll, and poly-ethylene-glycol at specific concentrations (Hartman et al., 1974; Albertsson and Birkenmeier, 1988) or the system containing the colloids dextran and polyethylene-glycol with the surfactant Triton X-100 (Ruan et al., 2006). Mace et al. (2012) reported mixtures with an even higher number of components (both colloids and surfactants) and a corresponding higher number of phases in equilibrium. They report systems of up to six phases, each enriched in one of the polymers and depleted in the others.

\subsection{Outline of the thesis}

In this thesis we aim to get a better understanding of the phase behavior of polydisperse and multi-component mixtures in solution. We approached this in a systematic way, starting with model systems of hard spheres (Chapters 2 to 4 ) and ending with a system of polydisperse 
macromolecules (Chapter 5).

In Chapter 2 we reviewed the theory of interactions in a simple system of a solute component in a solvent based on the second virial coefficient. We expanded on the theory of McMillan and Mayer (1945) to allow for systems of multiple distinguishable types of solute components. We describe the general equations used in the thesis that define the phase boundary, stability of the mixture, and critical point. We chose to first describe the existing theory in order to more easily explain the expressions we used in our calculations. In the same chapter we also introduced size polydispersity in an asymmetric binary mixture of hard spheres in solution. We investigated the position of the phase boundary, the spinodal, and the critical point. Next to that we looked into the fractionation of the polydisperse component upon demixing.

In the next chapter (Chapter 3) we looked into the influence of non-additive interaction on the phase behavior and stability of binary mixtures with some polydispersity or impurities. Just as in the previous chapter we investigated the position of the phase boundary, the spinodal, and the critical point. Next to that we looked into the fractionation of the polydisperse component upon demixing.

In Chapter 4 we study how the addition of a third component influences the phase behavior of a two component mixture of hard spheres in solution that demixes. We look into the phase behavior of the resulting three component mixture depending on the interaction said third component has with the other two components and their respective concentrations.

In Chapter $\mathbf{5}$ we apply the knowledge we gained from the previous chapters to a binary mixture of polydisperse colloids. We theoretically predict the phase behavior of the macromolecules polyethylene glycol (PEO) and dextran, taking their experimental osmometric second virial coefficients and experimentally measured size polydispersity into account. We compare the theoretical predictions to the experimental phase diagram (from previously published data).

Finally in the last chapter (Chapter 6) we discuss our results from the different chapters and place the current work in a wider context of the available scientific literature and discuss future research on multi-component and polydisperse mixtures in solution. 


\subsection{Bibliography}

P.-A. Aichinger, C. Schmitt, D. Z. Gunes, M. E. Leser, L. Sagalowicz, and M. Michel. Phase separation in food material design inspired by Nature: Or: What ice cream can learn from frogs. Current Opinion in Colloid and Interface Science, 28:56-62, 2017. ISSN 18790399. doi: 10.1016/j.cocis.2017.03.002.

P.-Å. Albertsson and G. Birkenmeier. Affinity separation of proteins in aqueous threephase systems. Analytical Biochemistry, 175(1):154-161, 1988. ISSN 10960309. doi: 10.1016/0003-2697(88)90373-9.

L. Bellier-Castella, H. Xu, and M. Baus. Phase diagrams of polydisperse van der Waals fluids. Journal of Chemical Physics, 113(18):8337-8347, 2000. ISSN 00219606. doi: $10.1063 / 1.1316007$.

T. Biben and J.-P. Hansen. Spinodal instability of suspensions of large spheres in a fluid of small spheres. Journal of Physics: Condensed Matter, 3(42):F65-F72, 1991. ISSN 0953-8984. doi: 10.1088/0953-8984/3/42/006.

T. Biben and J.-P. Hansen. Osmotic depletion, non-additivity and phase separation. Physica A: Statistical Mechanics and its Applications, 235(1-2):142-148, 1997. ISSN 03784371. doi: 10.1016/S0378-4371(96)00335-4.

X. L. Chu, A. D. Nikolov, and D. T. Wasan. Effects of Particle Size and Polydispersity on the Depletion and Structural Forces in Colloidal Dispersions. Langmuir, 12(21):5004-5010, 1996. ISSN 0743-7463. doi: 10.1021/la960359u.

R. L. Cotterman and J. M. Prausnitz. Flash calculations for continuous or semicontinuous mixtures by use of an equation of state. Industrial \& Engineering Chemistry Process Design and Development, 24(2):434-443, 1985. ISSN 0196-4305. doi: 10.1021/i200029a038.

R. L. Cotterman, R. Bender, and J. M. Prausnitz. Phase Equilibria for Mixtures Containing Very Many Components. Development and Application of Continuous Thermodynamics for Chemical Process Design. Industrial and Engineering Chemistry Process Design and Development, 24(1):194-203, 1985. ISSN 01964305. doi: 10.1021/i200028a033.

P. W. De Bont, G. M. Van Kempen, and R. Vreeker. Phase separation in milk protein and amylopectin mixtures. Food Hydrocolloids, 16(2):127-138, 2002. ISSN 0268005X. doi: 10.1016/S0268-005X(01)00070-4. 
B. P. C. Dewi, E. van der Linden, A. Bot, and P. Venema. Second order virial coefficients from phase diagrams. Food Hydrocolloids, 101:105546, 2020. ISSN 0268005X. doi: 10.1016/j.foodhyd.2019.105546.

M. Dijkstra. Phase behavior of nonadditive hard-sphere mixtures. Physical Review E Statistical Physics, Plasmas, Fluids, and Related Interdisciplinary Topics, 58(6):7523-7528, 1998. ISSN 1063651X. doi: 10.1103/PhysRevE.58.7523.

M. Dijkstra, R. van Roij, and R. Evans. Phase diagram of highly asymmetric binary hardsphere mixtures. Physical Review E - Statistical Physics, Plasmas, Fluids, and Related Interdisciplinary Topics, 59(5):5744-5771, 1999. ISSN 1063651X. doi: 10.1103/PhysRevE.59.5744.

M. W. Edelman, E. van der Linden, E. de Hoog, and R. H. Tromp. Compatibility of Gelatin and Dextran in Aqueous Solution. Biomacromolecules, 2(4):1148-1154, 2001. ISSN 1525-7797. doi: 10.1021/bm015545f.

M. W. Edelman, E. Van Der Linden, and R. H. Tromp. Phase separation of aqueous mixtures of poly(ethylene oxide) and dextran. Macromolecules, 36(20):7783-7790, 2003. ISSN 00249297. doi: 10.1021/ma0341622.

C. Ersch, L. L. C. Meijvogel, E. van der Linden, A. Martin, and P. Venema. Interactions in protein mixtures. part i: Second virial coefficients from osmometry. Food Hydrocolloids, 52:982 - 990, 2016a. ISSN 0268-005X.

C. Ersch, E. van der Linden, A. Martin, and P. Venema. Interactions in protein mixtures. Part II: A virial approach to predict phase behavior. Food Hydrocolloids, 52:991-1002, 2016b. doi: 10.1016/j.foodhyd.2015.07.021.

M. Fasolo and P. Sollich. Effects of polymer polydispersity on the phase behaviour of colloid-polymer mixtures. Journal of Physics: Condensed Matter, 17(6):797-812, 2005. ISSN 0953-8984. doi: 10.1088/0953-8984/17/6/002.

J. Gaube, R. Höchemer, B. Keil, and A. Pfennig. Polydispersity Effects in the System Poly(ethylene glycol) + Dextran + Water. Journal of Chemical and Engineering Data, 38(2):207-210, 1993. ISSN 15205134. doi: 10.1021/je00010a005.

D. Goulding and J. P. Hansen. Effects of size polydispersity on depletion interactions. Molecular Physics, 99(10):865-874, 2001. ISSN 00268976. doi: 10.1080/00268970010018657. 
A. Hartman, G. Johansson, and P. Albertsson. Partition of Proteins in a Three-Phase System. European Journal of Biochemistry, 46(1):75-81, 1974. ISSN 14321033. doi: 10.1111/j.1432-1033.1974.tb03598.x.

R. A. Heidemann. The Classical Theory of Critical Points. In Supercritical Fluids, pages 39-64. Springer Netherlands, 1994. doi: 10.1007/978-94-015-8295-7_2.

T. L. Hill. An Introduction to Statistical Thermodynamics. Dover Publications, New York, 1986. ISBN 9780486652429.

P. Hopkins and M. Schmidt. Binary non-additive hard sphere mixtures: Fluid demixing, asymptotic decay of correlations and free fluid interfaces. Journal of Physics Condensed Matter, 22(32), 2010. ISSN 09538984. doi: 10.1088/0953-8984/22/32/325108.

S. Ji and J. Y. Walz. Interaction potentials between two colloidal particles surrounded by an extremely bidisperse particle suspension. Journal of Colloid and Interface Science, 394 (1):611-618, 2013. ISSN 00219797. doi: 10.1016/j.jcis.2012.11.040.

C. H. Kang and S. I. Sandler. Effects of polydispersivity on the phase behavior of the aqueous two-phase polymer systems. Macromolecules, 21(10):3088-3095, 1988. ISSN 0024-9297.

V. Kontogiorgos, S. M. Tosh, and P. J. Wood. Phase behaviour of high molecular weight oat $\beta$-glucan/whey protein isolate binary mixtures. Food Hydrocolloids, 23(3):949-956, 2009. ISSN 0268005X. doi: 10.1016/j.foodhyd.2008.07.005.

M. Larsson and B. Mattiasson. Characterization of aqueous two-phase systems based on polydisperse phase forming polymers: Enzymatic hydrolysis of starch in a PEG-starch aqueous two-phase system. Biotechnology and Bioengineering, 31(9):979-983, 1988. ISSN 10970290. doi: 10.1002/bit.260310910.

H. N. Lekkerkerker and R. Tuinier. Colloids and the Depletion Interaction, volume 833. Springer, 2011. ISBN 978-94-007-1222-5. doi: 10.1007/978-94-007-1223-2.

C. R. Mace, O. Akbulut, A. A. Kumar, N. D. Shapiro, R. Derda, M. R. Patton, and G. M. Whitesides. Aqueous Multiphase Systems of Polymers and Surfactants Provide Self-Assembling Step-Gradients in Density. Journal of the American Chemical Society, 134(22):9094-9097, 2012. ISSN 0002-7863. doi: 10.1021/ja303183z.

W. G. McMillan and J. E. Mayer. The Statistical Thermodynamics of Multicomponent Systems. The Journal of Chemical Physics, 13(7):276-305, 1945. ISSN 0021-9606. doi: $10.1063 / 1.1724036$. 
P. Paricaud. Phase equilibria in polydisperse nonadditive hard-sphere systems. Physical Review E, 78(2):021202, 2008. ISSN 1539-3755. doi: 10.1103/PhysRevE.78.021202.

N. Park and J. C. Conrad. Phase behavior of colloid-polymer depletion mixtures with unary or binary depletants. Soft Matter, 13(15):2781-2792, 2017. ISSN 17446848. doi: 10.1039/C6SM02891H.

M. Piech and J. Y. Walz. Effect of Polydispersity and Charge Heterogeneity on the Depletion Interaction in Colloidal Systems. Journal of Colloid and Interface Science, 225(1):134-146, 2000. ISSN 00219797. doi: 10.1006/jcis.2000.6755.

M. Pojić, A. Mišan, and B. Tiwari. Eco-innovative technologies for extraction of proteins for human consumption from renewable protein sources of plant origin. Trends in Food Science and Technology, 75(March):93-104, 2018. ISSN 09242244. doi: 10.1016/j.tifs.2018.03.010.

R. Roth, R. Evans, and A. A. Louis. Theory of asymmetric nonadditive binary hard-sphere mixtures. Physical Review E-Statistical Physics, Plasmas, Fluids, and Related Interdisciplinary Topics, 64(5):13, 2001. ISSN 1063651X. doi: 10.1103/PhysRevE.64.051202.

K. Ruan, B. H. Wang, J. X. Xiao, and J. N. Tang. Interfacial tension of aqueous threephase systems formed by Triton X-100/PEG/Dextran. Journal of Dispersion Science and Technology, 27(7):927-930, 2006. ISSN 01932691. doi: 10.1080/01932690600766223.

W. F. Sager. Systematic study on the influence of impurities on the phase behavior of sodium bis(2-ethylhexyl) sulfosuccinate microemulsions. Langmuir, 14(22):6385-6395, 1998. ISSN 07437463. doi: 10.1021/la9709608.

A. Santos, M. López De Haro, and S. B. Yuste. Virial coefficients, thermodynamic properties, and fluid-fluid transition of nonadditive hard-sphere mixtures. Journal of Chemical Physics, 132(20), 2010. ISSN 00219606. doi: 10.1063/1.3429600.

R. P. Sear and J. a. Cuesta. Instabilities in complex mixtures with a large number of components. Physical Review Letters, 91(24):245701, 2003. ISSN 10797114. doi: 10.1103/PhysRevLett.91.245701.

R. P. Sear and D. Frenkel. Phase behavior of colloid plus polydisperse polymer mixtures. Physical Review E - Statistical Physics, Plasmas, Fluids, and Related Interdisciplinary Topics, 55(2):1677-1681, 1997. ISSN 1063651X. doi: 10.1103/PhysRevE.55.1677.

P. Sollich. Predicting phase equilibria in polydisperse systems. Journal of Physics Condensed Matter, 14(3):R79-R117, 2002. ISSN 09538984. doi: 10.1088/0953-8984/14/3/201. 
M. R. Stapleton, D. J. Tildesley, and N. Quirke. Phase equilibria in polydisperse fluids. The Journal of Chemical Physics, 92(7):4456-4467, 1990. ISSN 0021-9606. doi: $10.1063 / 1.457756$.

R. Tuinier and A. V. Petukhov. Polymer Polydispersity Effect on Depletion Interaction between Colloidal Particles. Macromolecular Theory and Simulations, 11(9):975-984, 2002. ISSN 10221344. doi: 10.1002/1521-3919(200211)11:9<975::AID-MATS975>3.0.CO;2-Y.

R. Tuinier, J. Rieger, and C. G. De Kruif. Depletion-induced phase separation in colloidpolymer mixtures. Advances in Colloid and Interface Science, 103(1):1-31, 2003. ISSN 00018686. doi: 10.1016/S0001-8686(02)00081-7.

S. L. Turgeon, M. Beaulieu, C. Schmitt, and C. Sanchez. Protein-polysaccharide interactions: Phase-ordering kinetics, thermodynamic and structural aspects. Current Opinion in Colloid and Interface Science, 8(4-5):401-414, 2003. ISSN 13590294. doi: 10.1016/S13590294(03)00093-1.

F. van de Velde, E. H. de Hoog, A. Oosterveld, and R. H. Tromp. Protein-Polysaccharide Interactions to Alter Texture. Annual Review of Food Science and Technology, 6(1): 371-388, apr 2015. ISSN 1941-1413. doi: 10.1146/annurev-food-022814-015558.

N. A. Verhaegh, J. S. van Duijneveldt, J. K. Dhont, and H. N. Lekkerkerker. Fluid-fluid phase separation in colloid-polymer mixtures studied with small angle light scattering and light microscopy. Physica A: Statistical Mechanics and its Applications, 230(3-4): 409-436, sep 1996. ISSN 03784371. doi: 10.1016/0378-4371(96)00145-8.

J. Y. Walz. Effect of Polydispersity on the Depletion Interaction between Colloidal Particles. Journal of Colloid and Interface Science, 178(2):505-513, 1996. ISSN 00219797. doi: 10.1006/jcis.1996.0145.

Z. Zhao, Q. Li, X. Ji, R. Dimova, R. Lipowsky, and Y. Liu. Molar mass fractionation in aqueous two-phase polymer solutions of dextran and poly(ethylene glycol). Journal of Chromatography $A, 1452: 107-115,2016$. ISSN 18733778. doi: 10.1016/j.chroma.2016.04.075. 



\section{Effect of polydispersity on the phase behavior of additive hard spheres in solution, part I}




\begin{abstract}
We study the theoretical phase behavior of an asymmetric binary mixture of hard spheres, of which the smaller component is monodisperse and the larger component is polydisperse. The interactions are modelled in terms of the second virial coefficient and are assumed to be additive hard sphere interactions. The polydisperse component is subdivided into sub-components and has an average size ten times the size of the monodisperse component. We give the set of equations that defines the phase diagram for mixtures with more than two components in a solvent. We calculate the theoretical liquid-liquid phase separation boundary (the binodal), the critical point and the spinodal. We vary the distribution of the polydisperse component in skewness, modality, polydispersity and number of sub-components. We compare the phase behavior of the polydisperse mixtures with binary monodisperse mixtures for the same average size and binary monodisperse mixtures for the same effective interaction. We find that the largest species in the larger (polydisperse) component causes the largest shift in the position of the phase boundary, critical point and spinodal compared to the binary monodisperse binary mixtures. The polydisperse component also shows fractionation. The smaller species of the polydisperse component favor the phase enriched in the smaller component. This phase also has a higher volume fraction compared to the monodisperse mixture.
\end{abstract}




\subsection{Introduction}

Binary mixtures are usually studied as mixtures of two monodisperse components, however components in nature are usually not that simple, and well defined. Often components will exhibit varying degrees of polydispersity in terms of their size, shape, and charge, which is often ignored when studying phase behavior.

Phase separation between hard spheres is driven by two different physical mechanisms. One mechanism involves only excluded volume interactions where the minimum distance between the particles is determined by the sum of their respective radii (Biben and Hansen, 1997), where only a certain asymmetry in the sizes of the particles in the mixture is necessary (Biben and Hansen, 1991). This asymmetry leads to depletion of small spheres around the large spheres and as a result to an effective attraction (depletion interaction) between the larger spheres (Dijkstra et al., 1999). This mechanism is referred to as additive hard sphere (HS) model. The case where the minimum distance between the particles is larger or smaller than the sum of their respective radii is referred to as non-additive hard sphere (NAHS) model. In both cases, upon phase separation, the mixture will demix into two (or more) phases, each enriched in one of the components. In this work we will focus on the first type, binary mixtures with significant asymmetry in their size.

In most studies on the phase behavior of binary mixtures the polydispersity of the components is ignored. However, from experiments with for example gelatin and dextran, it is found that polydispersity has an influence on the phase behavior. The polydispersity of both components leads to significant fractionation, especially for the dextran (Edelman et al., 2001).

Polydispersity has an effect on the depletion interaction. With increasing polydispersity, the repulsive barrier decreases, leading to an enhanced rate of flocculation of the large colloidal particles (Goulding and Hansen, 2001). Also Walz (1996) studied the depletion interaction in a solution of normally distributed macromolecules and showed that polydispersity can lead to increased flocculation through the formation of secondary potential energy minima. In later studies they found that polydispersity significantly lowered the magnitude of the repulsive structural barrier, which can be understood in terms of a change in depletion of the macromolecules from the gap (Piech and Walz, 2000). The polydispersity of the smaller component affects the pair potential between the large particles (Chu et al., 1996). This effect on the depletion interaction can stabilize the particle suspension in the short term, but will still destabilize over time (Tuinier and Petukhov, 2002).

Studying the phase behavior of polydisperse mixtures is challenging, since a polydisperse component effectively consists of a large number of sub-components, each with a different size and possibly also different shape or charge (Sollich, 2002). Some theoretical work has 
been done on predicting the phase behavior of polydisperse components. Cotterman and co-authors used continuous and semi-continuous distributions to predict the fluid-vapor phase diagram of polydisperse components (Cotterman et al., 1985; Cotterman and Prausnitz, 1985). Santos et al. (2010) studied the phase behavior of polydisperse compounds like polystyrene and polyethylene glycol. They found that the polymer polydispersity played a crucial role in the phase behavior: the broad size distributions lead to a wide range of depletion attractions giving rise to spinodal decomposition preventing gelation. BellierCastella et al. (2000) used a van der Waals approximation for the free energy to study the phase behavior of polydisperse fluids composed of spherical particles. They found the onset of a three phase co-existence at higher polydispersity. Fasolo and Sollich (2005) studied theoretically the equilibrium phase behavior of mixtures of polydisperse hard-sphere collids and monodisperse polymers based on the Asakura-Oosawa model. They found that with polydispersity significant fractionation occurred. Polydispersity delayed the onset of both gas-liquid and fluid-solid separation. Also Sear and Frenkel (1997) used the Asakura-Oosawa model to predict the phase behavior between a monodisperse colloid and a polydisperse polymer. They found that polydispersity increased the extent of the fluid-fluid co-existence. Warren (1999) studied the interaction between hard spheres with a bimodal size distribution and found that demixing caused additional size partitioning and fractionation. Kang and co-authors used a universal quasi-chemical (UNIQUAC) model to predict the phase behavior of aqueous polymer systems. They found that the polydispersity of the polymers enlarged the two-phase region considerably near the plait point and resulted in smaller miscibility regions far from the plait point. They also found that the average molecular weights of polymers in the phases differed significantly and this differences increased with larger polydispersity, due to this fractionation, the polydispersity of each polymer was smaller in each child phase compared to the parent mixture (Kang and Sandler, 1988). Others modelled the phase behavior of non-additive hard-sphere systems using Monte Carlo simulations (Paricaud, 2008). They found that with increasing polydispersity the miscibility region decreased and that the critical point shifted towards lower pressures. Also Stapleton and co-authors used Monte Carlo simulations to predict the phase behavior of mixtures with fixed or variable polydispersity (Stapleton et al., 1990). They found that mixtures even with a very small degree of polydispersity resulted in differences in the phase separation and the fractionation between the coexisting phases.

In this study we aim to get a better understanding of how size polydispersity influences the liquid-liquid phase behavior in binary mixtures, mainly on the position of the phase boundary, the spinodal, and the critical point. Next to that we aim to predict the fractionation of the polydisperse component between the phases. We model the interactions between the different components using the second virial coefficient. We limit the calculations to the 
second order virial coefficient, because the calculations form a stepping stone for expansion to non-additive interactions, polydispersity effects and multi-component mixtures. For a discussion on the relevancy of this second order virial approach we refer to Chapter 1.

We start the theoretical considerations with reviewing the interaction in a simple system of a solute in a solvent (Section 2.2.1). In Section 2.2.2 and Section 2.2.3 we expand the second virial coefficients for solutions with one type of solute component to solutions with multiple distinguishable types of solute components. Section 2.2.4 describes the theory about the stability of a mixture, Section 2.2.5 describes the theory about the critical point and finally Section 2.2.6 describes the theory about the phase boundary. We chose to first describe the existing theory in order to more easily explain the expressions we used in our calculations. With the expressions in Section 2.2 we calculate the phase behavior for different mixtures with varying polydispersity. In Section 2.3 we discuss the resulting phase diagrams, first in Section 2.3.1 we divided the polydisperse component into two sub-components and in Section 2.3.2 we increased the number of sub-components to nine. Finally we discuss fractionation of the polydisperse component in Section 2.3.3.

\subsection{Theory}

We start by deriving the equations of state for dilute solutions. Next we derive the virial expansion for solutions with one solute component. Subsequently we derive the second virial coefficient for mixtures with an aribtrary number of distinguishable components. This gives us all the parameters we need to define the stability boundary, the critical point and phase boundary of a mixture. The resulting system of equations is solved in Matlab R2017b.

\subsubsection{Dilute liquid solutions}

We consider a two component solution, in which one component is the solvent and the other component is the solute. We define $N_{s}$ as the number of solvent particles and $N_{\nu}$ as the number of solute particles in a volume $V$ at a temperature $T$. The total number of particles in the system is then $N=N_{\nu}+N_{s}$ and since we assume a dilute solution, $N_{s}>>N_{\nu}$. The system is in constant thermal contact with the environment and both the volume, and the number of solute and solvent particles are fixed (canonical ensemble) (Hill, 1986).

The sum of the kinetic $(K)$ and potential energies $(U)$ of the system represents the Hamiltonian $(H)$ of the system, given by

$$
H\left(p^{N_{\nu}+N_{s}}, q^{N_{\nu}+N_{s}}\right)=K\left(p^{N_{\nu}+N_{s}}\right)+U\left(r^{N_{\nu}+N_{s}}\right)
$$


in which

$$
\begin{aligned}
K\left(\vec{p}_{1} \ldots \vec{p}_{N_{\nu}+N_{s}}\right) & =\sum_{i=1}^{N_{\nu}} \frac{\left|\vec{p}_{i}\right|^{2}}{2 m}+\sum_{i=N_{\nu}+1}^{N_{\nu}+N_{s}} \frac{\left|\vec{p}_{i}\right|^{2}}{2 m_{s}} \\
U\left(\vec{r}_{1} \ldots \vec{r}_{N_{\nu}+N_{s}}\right) & =\sum_{i<j}^{N_{\nu}+N_{s}} \phi_{i j}\left(\vec{r}_{i}-\vec{r}_{j}\right) \\
& =\sum_{i<j}^{N_{\nu}} \phi_{i j}\left(\vec{r}_{i}-\vec{r}_{j}\right)+\sum_{i<j}^{N_{s}} \phi_{i j}\left(\vec{r}_{i}-\vec{r}_{j}\right)+\sum_{i}^{N_{\nu}} \sum_{j}^{N_{s}} \phi_{i j}\left(\vec{r}_{i}-\vec{r}_{j}\right) \\
& =U^{N_{\nu}}+U^{N_{s}}+U^{N_{\nu} N_{s}}
\end{aligned}
$$

where $\overrightarrow{p_{i}}$ is the impulse of particle $i, m_{\nu}$ is the mass of a solute particle and $m_{s}$ is the mass of a solvent particle, $\phi_{i j}$ is the pair potential between particle $i$ and $j$ and $\overrightarrow{r_{i}}$ is the position of particle $i$.

The canonical partition function $(Z)$ describes the statistical properties of the system for a given temperature, volume, and number of particles. The partition function is the sum of all the different individual energy states in which the system can exist. The states of the system are specified by both the position and the momentum of the particles. Applying the partition function to dilute solutions makes it possible to reduce the many-body problem in statistical mechanics to problems of one-body, two-body, three-body, etc.

$$
\begin{aligned}
Z\left(T, V, N_{\nu}, N_{s}\right)=\frac{h^{-\left(3 N_{\nu}+3 N_{s}\right)}}{N_{\nu} ! N_{s} !} \times \int_{V} \exp \left(-\beta H\left(p^{N_{\nu}}, p^{N_{s}}, q^{N_{\nu}}, q^{N_{s}}\right)\right) \\
\times d \vec{r}_{1} \ldots d \vec{r}_{N_{\nu}+N_{s}} d \vec{p}_{1} \ldots d \vec{p}_{N_{\nu}+N_{s}}
\end{aligned}
$$

where $h$ is Plank's constant and $\beta=\frac{1}{k T}$, in which $k$ is Boltzmann's constant.

Other thermodynamic variables, such as the Helmholtz free energy, the pressure and the chemical potential can be expressed in terms of this function or its derivatives. The Helmholtz free energy $(A)$ for this system is then given by (Hill, 1986):

$$
A\left(T, V, N_{\nu}, N_{s}\right)=-k T \ln \left(Z\left(T, V, N_{\nu}, N_{s}\right)\right)
$$

With the differential of the free energy given by:

$$
d A=-S d T-p d V+\sum_{i} \mu_{i} d N_{i}
$$

Therefore, the pressure $(p)$ is given by:

$$
p=-\left(\frac{\partial A}{\partial V}\right)_{T, N}=k T\left(\frac{\partial Z}{\partial V}\right)_{T, N}
$$


and the chemical potential $\left(\mu_{i}\right)$ for component $i$ is given by:

$$
\mu_{i}=\left(\frac{\partial A}{\partial N_{i}}\right)_{T, V, N_{\alpha \neq i}}=-k T\left(\frac{\partial Z}{\partial N_{i}}\right)_{T, V, N_{\alpha \neq i}}
$$

Since we focus on particles with hard sphere interaction, we can integrate out the momentum integrals in Eq. (2.4).

$$
\begin{aligned}
Z\left(T, V, N_{\nu}, N_{s}\right) & =\frac{\Lambda_{\nu}^{-3 N_{\nu}} \Lambda_{s}^{-3 N_{s}}}{N_{\nu} ! N_{s} !} \times \int_{V} d \vec{r}_{1} \ldots d \vec{r}_{N_{\nu}+N_{s}} \exp \left(-\beta U\left(\vec{r}_{1} \ldots \vec{r}_{N_{\nu}+N_{s}}\right)\right) \\
& =\Lambda_{\nu}^{-3 N_{\nu}} \Lambda_{s}^{-3 N_{s}} Q\left(T, V, N_{\nu}, N_{s}\right)
\end{aligned}
$$

where $\Lambda=\left(\frac{h^{2}}{2 \pi m k T}\right)^{1 / 2}$ is the mean thermal wavelength and $Q$ the configuration integral. The configuration integral is the integral over all possible configurations of the $N$ molecules in the system:

$$
Q\left(T, V, N_{\nu}, N_{s}\right)=\frac{1}{N_{\nu} ! N_{s} !} \int_{V} d \vec{r}_{1} \ldots d \vec{r}_{N_{\nu}+N_{s}} \times \exp \left(-\beta U\left(\vec{r}_{1}, \ldots, \vec{r}_{N_{\nu}+N_{s}}\right)\right)
$$

The first three configuration integrals are:

$$
\begin{aligned}
Q_{1} & =\int_{V} d \vec{r}_{1}=V \\
Q_{2} & =\frac{1}{2} \int_{V} d \vec{r}_{1} d \vec{r}_{2} \exp \left[-\beta \phi\left(\left|\vec{r}_{1}-\vec{r}_{2}\right|\right)\right] \\
Q_{3} & =\frac{1}{6} \int_{V} d \vec{r}_{1} d \vec{r}_{2} d \vec{r}_{3} \\
& \times \exp \left\{-\beta\left[\phi\left(\left|\vec{r}_{1}-\vec{r}_{2}\right|\right)+\phi\left(\left|\vec{r}_{1}-\vec{r}_{3}\right|\right)+\phi\left(\left|\vec{r}_{2}-\vec{r}_{3}\right|\right)\right]\right\}
\end{aligned}
$$

The configuration integral $Q_{1}$ indicates there is only one particle present in our selected volume. $Q_{2}$ indicates there are two particles present in our selected volume: interacting or not interaction. $Q_{3}$ indicates there are three particles present in the volume. These particles can interact with each other or not interact. The number of combinations of interactions increases significantly when increasing the number of particles.

The configuration integrals can be represented in diagrams where each dot is a particle present in the volume and a line between the dots indicates interaction: 


$$
\begin{aligned}
Q_{1} & =\bullet \\
Q_{2} & =\frac{1}{2}(\bullet \bullet+\bullet \bullet) \\
Q_{3} & =\frac{1}{6}(\bullet \bullet+3 \bullet \bullet+3 \bullet \bullet+\bullet
\end{aligned}
$$

In our analysis we consider solutions where the solvent particles are present in a much larger number than the solute particles. This means that solute particles have relatively low influence on the statistics of the solvent particles. Following the MacMillan - Mayer theory we can describe the interactions between the solute particles by a potential of mean force equation and thus can apply additivity of the particle-particle interactions in Eq. (2.3) (McMillan and Mayer, 1945).

$U\left(\vec{r}_{1}, \ldots, \vec{r}_{N_{\nu}+N_{s}}\right)=U_{N_{\nu} N_{\nu}}\left(\vec{r}_{1}, \ldots, \vec{r}_{N_{\nu}}\right)+U_{N_{\nu} N_{s}}\left(\vec{r}_{1}, \ldots, \vec{r}_{N_{\nu}+N_{s}}\right)+U_{N_{s} N_{s}}\left(\vec{r}_{N_{\nu}+1}, \ldots, \vec{r}_{N_{\nu}+N_{s}}\right)$

The potential of mean force for dissolved particles $(W)$ is defined according to:

$$
\exp \left(-\beta W\left(\vec{r}_{1}, \ldots, \vec{r}_{N_{\nu}}\right)\right)=\frac{\int d \vec{r}_{N_{\nu}+1}, \ldots, d \vec{r}_{N_{\nu}+N_{s}} \exp \left(-\beta U\left(\vec{r}_{1}, \ldots, \vec{r}_{N_{\nu}+N_{s}}\right)\right)}{\left(\begin{array}{c}
\int d \vec{r}_{N_{\nu}+1}, \ldots, d \vec{r}_{N_{\nu}+N_{s}} \\
\times \exp \left(-\beta U_{N_{s} N_{s}}\left(\vec{r}_{N_{\nu}+1}, \ldots, \vec{r}_{N_{\nu}+N_{s}}\right)\right)
\end{array}\right)}
$$

Using Eqs. (2.11) and (2.19), the configuration integral becomes (Vafaei et al., 2014):

$$
\begin{aligned}
Q\left(T, V, N_{\nu}, N_{s}\right)= & \frac{1}{N_{\nu} !} \int d \vec{r}_{1} \ldots d \vec{r}_{N} \exp \left[-\beta W_{\nu}\left(\vec{r}_{1}, \ldots, \vec{r}_{N_{\nu}}\right)\right] \times \frac{1}{N_{s} !} \int d \vec{r}_{N_{\nu}+1} \ldots d \vec{r}_{N_{\nu}+N_{s}} \\
& \quad \times \exp \left[-\beta U_{N_{s} N_{s}}\left(\vec{r}_{N+1}, \ldots, \vec{r}_{N+N_{s}}\right)\right] \\
= & Q_{W}\left(T, V, N_{\nu}, \mu_{s}\right) Q_{s}\left(T, V, N_{s}\right)
\end{aligned}
$$

The Helmholtz free energy (Eq. (2.5)) of the system then becomes the sum of the Helmholtz free energy of the solvent and the Helmholtz free energy of the solute:

$$
A\left(T, V, N_{\nu}, N_{S}\right)=-k T \ln \left(Z_{W}\left(T, V, N_{\nu}, \mu_{s}\right)\right)-k T \ln \left(Z_{s}\left(T, V, N_{s}\right)\right)
$$

\subsubsection{Second virial coefficient of a dilute solution with a single solute component}

Similar to the expansion of the universal gas law by a virial expansion for real gasses, we can write a virial expansion for the osmotic pressure, $\Pi$, of a solution according to: 


$$
\beta \Pi=\rho+B_{2}\left(T, \mu_{s}\right) \rho^{2}+B_{3}\left(T, \mu_{s}\right) \rho^{3}+\ldots
$$

with $\rho$ the number density of the component $\left(\frac{N_{\nu}}{V}\right), B_{2}$ the second virial coefficient, and $B_{3}$ the third virial coefficient. The second virial coefficient accounts for the increase in osmotic pressure due to particle pairwise interaction. The third virial coefficient accounts for the interaction between three particles. The equation can be expanded for higher densities with $B_{n}$, the $n^{\text {th }}$ virial coefficient, which accounts for the interaction between $n$ different particles.

Until now we have been using the canonical ensemble to describe the system. In the canonical ensemble, the number of particles $\left(N_{\nu}+N_{s}\right)$, the temperature $(T)$, and the volume $(V)$ are fixed. The restraint of constant number of particles becomes tedious when accounting for the interaction between the different particles, therefore we will use the grand canonical ensemble to derive the virial coefficients. In the grand canonical ensemble, the temperature $(T)$ and the volume $(V)$ are fixed, as well as the chemical potentials $\left(\mu_{i}\right)$.

We can write the grand canonical partition function independent on $N_{\nu}$ and $N_{s}$ by performing a transformation of the number of compounds by the chemical potential $\mu_{\nu}$ and $\mu_{s}$ (Hill, 1986).

$$
\begin{aligned}
\Xi\left(T, V, \mu_{\nu}, \mu_{s}\right) & =\sum_{N_{\nu}=0}^{\infty} e^{\beta \mu_{\nu} N_{\nu}} \times \sum_{N_{s}=0}^{\infty} e^{\beta \mu_{s} N_{s}} Z\left(T, V, N_{\nu}, N_{s}\right) \\
& =\sum_{N_{\nu}=0}^{\infty} e^{\beta \mu_{\nu} N_{\nu}} Z_{W}\left(T, V, N_{\nu}, \mu_{s}\right) \times \Xi_{s}\left(T, V, \mu_{s}\right) \\
& =\Xi_{W}\left(T, V, \mu_{\nu}, \mu_{s}\right) \Xi_{s}\left(T, V, \mu_{s}\right)
\end{aligned}
$$

The equation of state for the system is given by:

$$
\beta(p+\Pi)=\frac{\ln \left(\Xi\left(T, V, \mu_{\nu}, \mu_{s}\right)\right)}{V}=\frac{\ln \left(\Xi_{s}\left(T, V, \mu_{s}\right)\right)}{V}+\frac{\ln \left(\Xi_{W}\left(T, V, \mu_{\nu}, \mu_{s}\right)\right)}{V}
$$

In which the osmotic pressure is given by:

$$
\beta \Pi=\frac{\ln \left(\Xi_{W}\left(T, V, \mu_{\nu}, \mu_{s}\right)\right)}{V}
$$

We can now define the activity as $z \equiv \frac{e^{\beta \mu}}{\Lambda^{3}}$

$$
\Xi_{W}\left(T, V, z_{\nu}, \mu_{s}\right)=\sum_{N=0}^{\infty} Q_{N}\left(T, V, N_{\nu}, \mu_{s}\right) z_{\nu}^{N}=1+Q_{1} z+Q_{2} z^{2}+Q_{3} z^{3}+\ldots
$$


The osmotic pressure can be written in terms of the logarithm of the grand canonical ensemble.

$$
\begin{aligned}
\beta \Pi\left(T, V, z_{\nu}, \mu_{s}\right) V & =\ln \left(\Xi_{W}\left(T, V, z_{\nu}, \mu_{S}\right)\right) \\
& =\ln \left(1+Q_{1} z+Q_{2} z^{2}+Q_{3} z^{3}+\ldots\right) \\
& =Q_{1} z+\left(Q_{2}-\frac{1}{2} Q_{1}^{2}\right) z^{2}+\left(Q_{3}-Q_{1} Q_{2}+\frac{1}{3} Q_{1}^{3}\right) z^{3}+\ldots
\end{aligned}
$$

This can be written as:

$$
\beta \Pi=\sum_{l=1}^{\infty} b_{l} z^{l}
$$

The coefficient $b_{l}$ is also known as the cluster-integral and indicate interaction among $l$ compounds.

$$
\begin{aligned}
& b_{1}=\frac{Q_{1}}{V} \\
& b_{2}=\frac{Q_{2}-\frac{1}{2} Q_{1}^{2}}{V} \\
& b_{3}=\frac{Q_{3}-Q_{1} Q_{2}+\frac{1}{3} Q_{1}^{3}}{V}
\end{aligned}
$$

Just as that configuration integrals can be written in diagrams (see Eqs. (2.15) to (2.17)), the cluster integrals can also be written as diagrams (Hansen and McDonald, 2013):

$$
\begin{aligned}
& b_{1}=\frac{1}{V}(\bullet) \\
& b_{2}=\frac{1}{2 V}(\bullet \bullet) \\
& b_{3}=\frac{1}{6 V}(3 \bullet \bullet+\bullet)
\end{aligned}
$$

Two particle interactions can be defined by the Mayer f-function:

$$
f_{12}=\exp \left(-\beta W_{12}\right)-1
$$

and can be represented by: 
Substituting the Mayer f-function into the cluster integrals we get:

$$
\begin{aligned}
& b_{1}=\frac{1}{V} \int_{V} d \vec{r}_{1}=1 \\
& b_{2}=\frac{1}{2 V} \int_{V} d \vec{r}_{1} d \vec{r}_{2} f_{12}=2 \pi \int_{0}^{\infty} d r r^{2} f(r) \\
& b_{3}=2 b_{2}^{2}+\frac{1}{6} \int d \vec{r} \int d \vec{r}^{\prime} f(r) f\left(r^{\prime}\right) f\left(\left|\vec{r}-\vec{r}^{\prime}\right|\right)
\end{aligned}
$$

In order to find the relationship between cluster integrals and the virial coeffcients, we need to do several substitutions and inversions using also Eq. (2.28):

$$
\rho=\frac{N}{V}=\beta z\left(\frac{\partial \Pi}{\partial z}\right) T=\sum_{l=1}^{\infty} l b_{l} z^{l}=z+2 b_{2} z^{2}+3 b_{3} z^{3}+\ldots
$$

Inverting the series we obtain for the activity (Abramowitz and Stegun, 1972):

$$
z=\rho-2 b_{2} \rho^{2}+\left(8 b_{2}^{2}-3 b_{3}\right) \rho^{3}+\ldots
$$

This can be substituted in the equation for the pressure (Eq. (2.28)):

$$
\beta \Pi=\rho-b_{2} \rho^{2}+2\left(2 b_{2}^{2}-b_{3}\right) \rho^{3}+\ldots
$$

Comparing this equation to Eq. (2.22), we see that the second virial coefficient is equivalent to $-b_{2}$ :

$$
\begin{aligned}
B\left(T, \mu_{s}\right) & =-b_{2}=-\frac{1}{2 V}(\bullet \bullet) \\
& =2 \pi \int_{0}^{\infty} d r r^{2}(1-\exp (-\beta W(r))) \\
& =-2 \pi \int_{0}^{\infty} d r r^{2} f(r)
\end{aligned}
$$

For hard spheres we define the interaction potentials as for components with a diameter $\sigma:$

$$
W(r)_{H S}= \begin{cases}0, & r>\sigma \\ \infty, & r \leq \sigma\end{cases}
$$

The second virial coefficient then becomes:

$$
B\left(T, \mu_{s}\right)=\frac{2 \pi}{3}(\sigma)^{3}
$$




\subsubsection{Second virial coefficient of solutions with multiple solute compounds}

When there are more distinct compounds $\left(\nu_{1}, . ., \nu_{n}\right)$ in the solution with $n$ the total number of distinguishable compounds, or species, there are two main types of two particle interactions that can occur:

- interactions between indistinguishable components, i.e. components of the same species:

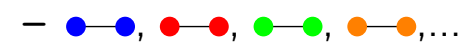

- interactions between distinguishable components, i.e. components of different species:

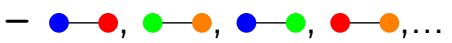

We can write the configuration integral $Q_{N}$ in general as:

$Q_{N}=\frac{1}{N_{\nu_{1}} ! \ldots N_{\nu_{n}} !} \times \int_{V} d \vec{r}_{1_{\nu_{1}}}, \ldots, \vec{r}_{N_{\nu_{1}}}, \ldots, \vec{r}_{1_{\nu_{n}}}, . ., \vec{r}_{N_{\nu_{n}}} \times \exp \left[-\beta \sum_{i<j}^{N} W_{x y}\left(r_{i j}\right)\right]$

In which: $N=\sum_{i}^{n} N_{\nu_{i}}$ or the total number of particles in the configuration and $x$ and $y$ can be of any type $\nu_{n}$ in the mixture.

The general equation for the partition function in the grand canonical ensemble then becomes:

$$
\Xi\left(T, V, z_{\nu_{1}}, \ldots, z_{\nu_{n}}, \mu_{s}\right)=\sum_{N_{\nu_{1}}, \ldots, N_{\nu_{n}} \geqslant 0} Q_{N}(V, T) z_{\nu_{1}}^{N_{\nu_{1}}} \ldots z_{\nu_{n}}^{N_{\nu_{n}}}
$$

In the case of two particle interaction we have interaction between components of the same species and interaction between components of different species, so we obtain for $Q_{2}$ two types of configuration integrals (comparable to Eq. (2.13)):

$$
\begin{aligned}
Q_{2, x x} & =\frac{1}{2 !} \int_{V} d \vec{r}_{1_{x}} d \vec{r}_{2_{x}} \exp \left[-\beta W_{x x}\left(r_{1_{x}} r_{2_{x}}\right)\right] \\
& =\frac{1}{2} V^{2}+2 \pi V \int_{0}^{\infty} d r r^{2} f_{x}(r) \\
Q_{2, x y} & =\frac{1}{1 ! 1 !} \int_{V} d \vec{r}_{1_{x}} d \vec{r}_{2_{y}} \exp \left[-\beta W_{x y}\left(r_{1_{x}} r_{2_{y}}\right)\right] \\
& =2\left(\frac{1}{2} V^{2}+2 \pi V \int_{0}^{\infty} d r r^{2} f_{x y}(r)\right)
\end{aligned}
$$


In which $x$ and $y$ can be of any type $\nu_{n}$ in the mixture and $y \neq x$.

Also for the cluster integrals we obtain two types of integrals (comparable to Eq. (2.36)):

$$
\begin{aligned}
b_{2, x x} & =2 \pi \int_{0}^{\infty} d r r^{2}\left(\exp \left[-\beta W_{x x}\right]-1\right) \\
& =2 \pi \int_{0}^{\infty} d r r^{2} f_{x}(r) \\
b_{2, x y} & =4 \pi \int_{0}^{\infty} d r r^{2}\left(\exp \left[-\beta W_{x y}\right]-1\right) \\
& =2\left(2 \pi \int_{0}^{\infty} d r r^{2} f_{x y}(r)\right)
\end{aligned}
$$

In which $x$ and $y$ can be of any type $\nu_{n}$ in the mixture and $y \neq x$.

The general equation for the osmotic pressure becomes then (comparable to Eq. (2.38)):

$$
\begin{aligned}
\beta \Pi & =b_{1, \nu_{1}} z_{\nu_{1}}+b_{1, \nu_{2}} z_{\nu_{2}}+b_{1, \nu_{3}} z_{\nu_{3}}+\ldots+b_{2, \nu_{1} \nu_{1}} z_{\nu_{1}}^{2}+b_{2, \nu_{1} \nu_{2}} z_{\nu_{1}} z_{\nu_{2}}+b_{2, \nu_{1} \nu_{3}} z_{\nu_{1}} z_{\nu_{3}}+\ldots \\
& =\sum_{i}^{n} b_{1, \nu_{i}} z \nu_{i}+\sum_{i}^{n} b_{2, \nu_{i} \nu_{i}} z_{\nu_{i}}^{2}+\sum_{i<j}^{n} b_{2, \nu_{i} \nu_{j}} z_{\nu_{i}} z_{\nu_{j}}+\ldots
\end{aligned}
$$

For the second virial coefficient we obtain two types (comparable to Eq. (2.41)):

$$
\begin{aligned}
B_{x x}^{*} & =2 \pi \int_{0}^{\infty} d r r^{2}\left(1-\exp \left[-\beta W_{x x}\right]\right) \\
& =-2 \pi \int_{0}^{\infty} d r r^{2} f_{x}(r)=B_{x x} \\
B_{x y}^{*} & =4 \pi \int_{0}^{\infty} d r r^{2}\left(1-\exp \left[-\beta W_{x y}\right]\right) \\
& =2\left(-2 \pi \int_{0}^{\infty} d r r^{2} f_{x y}(r)\right)=2 B_{x y}
\end{aligned}
$$

In which $x$ and $y$ can be of any type $\nu_{n}$ in the mixture and $y \neq x$. Note: we define a $B^{*}$ to have all the second virial equations of the same form: $-2 \pi \int_{0}^{\infty} d r r^{2} f(r)$, with $f(r)$ dependent on the type of interaction.

For additive hard sphere interaction, the interaction potential for particles of different species is given by:

$$
W(r)_{H S}= \begin{cases}0, & r>\sigma_{i j} \\ \infty, & r \leq \sigma_{i j}\end{cases}
$$

with $\sigma_{i j}=\left(\sigma_{i}+\sigma_{j}\right) / 2$, the distance between the centers of the two components. When the interaction is not additive, the distance of closest approach of the centers of the two components becomes: $\sigma_{i j}=1 / 2\left(\sigma_{i}+\sigma_{j}\right)(1+\Delta)$, in which $\Delta$ accounts for the non-additivity of the interaction between the particles that are different. 
For the second virial coefficient we find (comparable to Eq. (2.43)):

$$
\begin{aligned}
& B_{x x}=\frac{2 \pi}{3}\left(\sigma_{x}\right)^{3} \\
& B_{x y}=\frac{2 \pi}{3}\left(\left(\frac{\sigma_{x}+\sigma_{y}}{2}\right)(1+\Delta)\right)^{3}
\end{aligned}
$$

(Again, for additive hard sphere interactions, $\Delta=0$ ).

The general equation for the osmotic pressure for a dilute mixture is then given by:

$$
\begin{aligned}
\beta \Pi & =\rho+B_{\nu_{1} \nu_{1}} \rho_{\nu_{1}}^{2}+2 B_{\nu_{1} \nu_{2}} \rho_{\nu_{1}} \rho_{\nu_{2}}+2 B_{\nu_{1} \nu_{3}} \rho_{\nu_{1}} \rho_{\nu_{3}} \ldots \\
& =\rho+\sum_{i}^{n} \sum_{j}^{n} B_{\nu_{i} \nu_{j}} \rho_{\nu_{i}} \rho_{\nu_{j}}+\ldots
\end{aligned}
$$

The second virial coefficients can be represented in matrix form:

$$
B=\left[\begin{array}{ccc}
B_{11} & \cdots & B_{1 n} \\
\vdots & \ddots & \vdots \\
B_{1 n} & \cdots & B_{n n}
\end{array}\right]
$$

In which we abbreviate the notation $B_{\nu_{i} \nu_{j}}$ to $B_{i j}$, and similarly, the densities $\rho_{\nu_{i}}$ by $\rho_{i}$.

\subsubsection{Stability of a mixture}

The stability of a mixture is dependent on the second derivative of the free energy. If the second derivative of the mixture becomes zero, the mixture is at the boundary of becoming unstable. Unstable mixtures have a negative second derivative (Beegle et al., 1974; Heidemann, 1975).

The free energy of a mixture is given by (Hill, 1986):

$$
A\left(T, V, N_{\nu_{1}}, \ldots, N_{\nu_{n}}, \mu_{s}\right)=-k T \ln \left(Z\left(T, V, N_{\nu_{1}}, \ldots, N_{\nu_{n}}, \mu_{s}\right)\right)
$$

and the differential is given by:

$$
d A=-S d T-p d V+\sum_{i}^{n} \mu_{i} d N_{i}
$$

in which the chemical potential (the first partial derivative of the free energy with respect to number of particles $\left.\left(N_{i}\right)\right)$ for component $i$ is given by:

$$
\mu_{i}=\mu_{i}^{0}+k T \ln \left(\rho_{i}\right)+2 k T\left(\sum_{j}^{n} B_{i j} \rho_{j}\right)
$$


For a mixture with $n$ distinguishable components, this second partial derivatives can be represented by a $n \times n$ matrix of the first partial derivatives of the chemical potential of each component.

This results in the following general stability matrix:

$$
\begin{aligned}
M_{1}= & {\left[\begin{array}{ccc}
\frac{\partial \mu_{1}}{\partial N_{1}} & \cdots & \frac{\partial \mu_{1}}{\partial N_{n}} \\
\vdots & \ddots & \vdots \\
\frac{\partial \mu_{n}}{\partial N_{1}} & \cdots & \frac{\partial \mu_{n}}{\partial N_{n}}
\end{array}\right] } \\
= & {\left[\begin{array}{ccc}
2 B_{11}+\frac{1}{\rho_{1}} & \cdots & 2 B_{1 n} \\
\vdots & \ddots & \vdots \\
2 B_{1 n} & \cdots & 2 B_{n n}+\frac{1}{\rho_{n}}
\end{array}\right] }
\end{aligned}
$$

When this matrix is positive definite, the mixture is stable (Solokhin et al., 2002). Based on this criterium, when one of the eigenvalues is not positive, the mixture becomes unstable. When the matrix has one zero eigenvalue and is otherwise positive definite, the mixture is on the spinodal and is at the limit of stability (Heidemann and Khalil, 1980).

In case of a binary mixture $(n=2)$, the spinodal is also equal to the determinant of matrix $M_{1}$. For mixtures with more components, this is not always the case anymore, as with an increasing number of components, there are an increasing number of eigenvalues for matrix $M_{1}$ that can become zero (Solokhin et al., 2002). This can be resolved by checking if the stability matrix is positive definite for small changes of in the concentrations of the components near the concentration where the $\operatorname{det}\left(M_{1}\right)$ is zero.

The determinant of matrix $M_{1}$ for a monodisperse binary mixture is given by:

$$
\operatorname{det}\left(M_{1}\right)=4\left(B_{11} B_{22}-B_{12}^{2}\right) \rho_{1} \rho_{2}+2 B_{11} \rho_{1}+2 B_{22} \rho_{2}+1
$$

Often however, components are not a 100\% monodisperse. Let's now investigate how the equation for the spinodal of the mixture changes when we introduce polydispersity in one of the components. We define a binary mixture in which one component (component 2 ) is polydisperse. The concentration of each of the particles in the polydisperse component can be represented by:

$$
\rho_{2}=\left[\begin{array}{lll}
x_{1} & \cdots & x_{m}
\end{array}\right] \times \rho_{2_{t o t}}
$$

with 


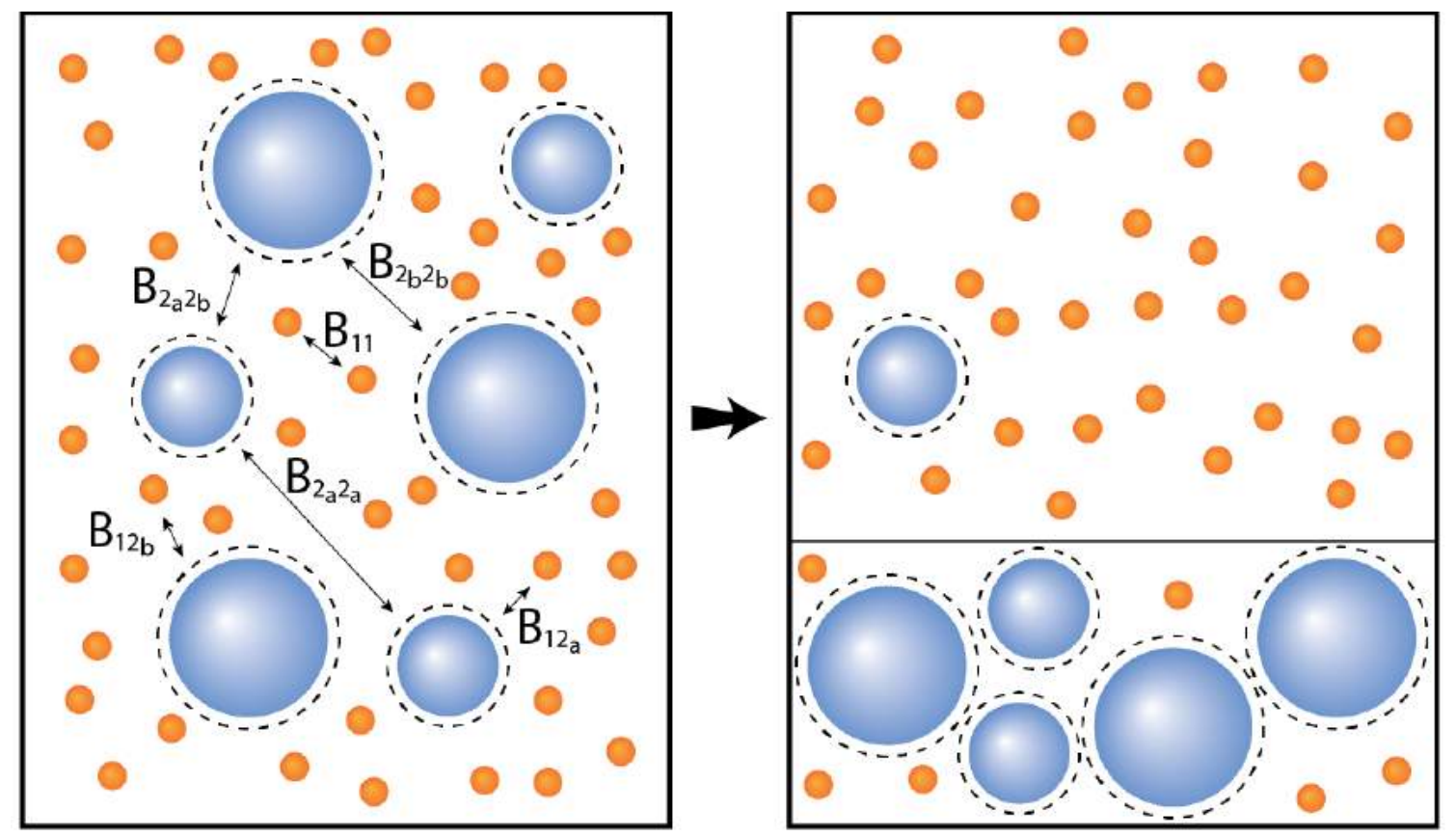

Figure 2.1: Graphical representation of a simple polydisperse mixture, in which the polydisperse component consists out of two sub-components ( $a$ and $b, n=3$ ), second virial coefficients are indicated. The mixture demixes into two phases, one phase enriched in the small component, one phase enriched in the large polydipserse component.

$$
\rho_{2_{\text {tot }}}=\sum_{i}^{m} \rho_{2_{i}}
$$

and

$$
x=x_{1}+\cdots+x_{m}=1
$$

Each of the components in this mixture has a corresponding virial coefficient and cross virial coefficient.

$$
B=\left[\begin{array}{ccc}
B_{11} & \cdots & B_{1 m} \\
\vdots & \ddots & \vdots \\
B_{1 m} & \cdots & B_{m m}
\end{array}\right]
$$

Let us investigate the equations for a simple polydisperse mixture, in which the polydisperse component consists out of two sub-components ( $a$ and $b, n=3$ ) (Figure 2.1). For the density and for the virial coefficient matrix we obtain:

$$
\rho_{2}=\left[\begin{array}{ll}
x_{a} & x_{b}
\end{array}\right] \times \rho_{2_{t o t}}
$$

with 


$$
\begin{gathered}
x=x_{a}+x_{b} \\
B=\left[\begin{array}{ccc}
B_{11} & B_{12_{a}} & B_{12_{b}} \\
B_{12_{a}} & B_{2_{a} 2_{a}} & B_{2_{a} 2_{b}} \\
B_{12_{b}} & B_{2_{a} 2_{b}} & B_{2_{b} 2_{b}}
\end{array}\right]
\end{gathered}
$$

The stability matrix becomes:

$$
M_{1}=\left[\begin{array}{ccc}
2 B_{11}+\frac{1}{\rho_{1}} & 2 B_{12_{a}} & 2 B_{12_{b}} \\
2 B_{12_{a}} & 2 B_{2_{a} 2_{a}}+\frac{1}{\rho_{2_{a}}} & 2 B_{2_{a} 2_{b}} \\
2 B_{12_{b}} & 2 B_{2_{a} 2_{b}} & 2 B_{2_{b} 2_{b}}+\frac{1}{\rho_{2_{b}}}
\end{array}\right]
$$

This results in the following determinant for the stability matrix:

$$
\begin{aligned}
\operatorname{det}\left(M_{1}\right)= & 2 B_{11} \rho_{1}+2 x_{a} B_{2_{a} 2_{a}} \rho_{2}+2 x_{b} B_{2_{b} 2_{b}} \rho_{2}-4 x_{a} B_{12_{a}}^{2} \rho_{1} \rho_{2}-4 x_{b} B_{12_{b}}^{2} \rho_{1} \rho_{2} \\
& -4 x_{a} x_{b} B_{2_{a} 2_{b}}^{2} \rho_{2}^{2}+4 x_{a} B_{11} B_{2_{a} 2_{a}} \rho_{1} \rho_{2}+4 x_{b} B_{11} B_{2_{b} 2_{b}} \rho_{1} \rho_{2} \\
& +4 x_{a} x_{b} B_{2_{a} 2_{a}} B_{2_{b} 2_{b}} \rho_{2}^{2}-8 x_{a} x_{b} B_{11} B_{2_{a} 2_{b}}^{2} \rho_{1} \rho_{2}^{2} \\
& -8 x_{a} x_{b} B_{12_{b}}^{2} B_{2_{a} 2_{a}} \rho_{1} \rho_{2}^{2}-8 x_{a} x_{b} B_{12_{a}}^{2} B_{2_{b} 2_{b}} \rho_{1} \rho_{2}^{2} \\
& +16 x_{a} x_{b} B_{12_{a}} B_{12_{b}} B_{2_{a} 2_{b}} \rho_{1} \rho_{2}^{2}+8 x_{a} x_{b} B_{11} B_{2_{a} 2_{a}} B_{2_{b} 2_{b}} \rho_{1} \rho_{2}^{2}+1
\end{aligned}
$$

With increasing number of sub-components, the number of terms in this determinant increases rapidly. This forms an incentive to try and treat the polydisperse component as if it is effectively one component. A natural and convenient choice for this route is coupled to the experimental determination of virial coefficients using membrane osmometry (Ersch et al., 2016). Namely, membrane osmometry yields values that are number averaged. Thus we choose number averaged virial coefficients.

The number averaged virial coefficient of a mixture can be written as:

$$
\begin{aligned}
B_{\text {mix }} & =B_{11} x_{1}^{2}+2 B_{12} x_{1} x_{2}+2 B_{13} x_{1} x_{3} \ldots \\
& =\sum_{i}^{m} \sum_{j}^{m} B_{i j} x_{i} x_{j}
\end{aligned}
$$

in which $B_{i i}$ is the second virial coefficient of the $i^{\text {th }}$ particle, $B_{i j}$ is the second cross virial coefficient of the $i^{\text {th }}$ particle and the $j^{\text {th }}$ particle, and $x_{i}$ is the fraction of the $i^{\text {th }}$ particle, $\sum x_{i}=1$. 
Using this definition, we can map the polydisperse mixture by a $2 \times 2$ matrix of virial coefficients. We will refer to this $2 \times 2$ matrix of effective virial coefficients.

$$
\begin{aligned}
B_{11_{\text {eff }}}= & B_{11} \\
B_{12_{\text {eff }}}= & \sum_{i}^{m} B[1 ; 1, \cdots, m](i) x_{i} \\
B_{22_{\text {eff }}}= & \sum_{i}^{m} \sum_{j}^{m} x_{i} B[1, \cdots, m ; 1, \cdots, m](i j) x_{j} \\
& B_{\text {eff }}=\left[\begin{array}{ll}
B_{11_{\text {eff }}} & B_{12_{\text {eff }}} \\
B_{12_{\text {eff }}} & B_{22_{\text {eff }}}
\end{array}\right]
\end{aligned}
$$

For the mixture we considered in Eq. (2.64), the effective virial coefficients become:

$$
\begin{aligned}
& B_{11_{\text {eff }}}=B_{11} \\
& B_{12_{e f f}}=x_{a} B_{12_{a}}+x_{b} B_{12_{b}} \\
& B_{22_{e f f}}=x_{a}^{2} B_{2_{a} 2_{a}}+2 x_{a} x_{b} B_{2_{a} 2_{b}}+x_{b}^{2} B_{2_{b} 2_{b}}
\end{aligned}
$$

The effective stability matrix for this mixture becomes then:

$$
M_{1_{\text {eff }}}=\left[\begin{array}{cc}
2 B_{11_{\text {eff }}+\frac{1}{\rho_{1}}} & 2 B_{12_{e f f}} \\
2 B_{12_{\text {eff }}} & 2 B_{22_{e f f}}+\frac{1}{\rho_{2}}
\end{array}\right]
$$

and the determinant then becomes:

$$
\begin{aligned}
\operatorname{det}\left(M_{1_{e f f}}\right)= & 4\left(B_{11_{e f f}} B_{22_{e f f}}-B_{12_{e f f}}^{2}\right) \rho_{1} \rho_{2}+2 B_{11_{e f f}} \rho_{1}+2 B_{22_{e f f}} \rho_{2}+1 \\
= & 4\left(B_{11}\left(x_{a}^{2} B_{2_{a} 2_{a}}+2 x_{a} x_{b} B_{2_{a} 2_{b}}+x_{b}^{2} B_{2_{b} 2_{b}}\right)-\left(x_{a} B_{12_{a}}+x_{b} B_{12_{b}}\right)^{2}\right) \rho_{1} \rho_{2} \\
& +2 B_{11} \rho_{1}+2\left(x_{a}^{2} B_{2_{a} 2_{a}}+2 x_{a} x_{b} B_{2_{a} 2_{b}}+x_{b}^{2} B_{2_{b} 2_{b}}\right) \rho_{2}+1 \\
= & 2 B_{11} \rho_{1}+2 x_{a}^{2} B_{2_{a} 2_{a}} \rho_{2}+2 x_{b}^{2} B_{2_{b} 2_{b}} \rho_{2}-4 x_{a}^{2} B_{12_{a}}^{2} \rho_{1} \rho_{2}-4 x_{b}^{2} B_{12_{b}}^{2} \rho_{1} \rho_{2} \\
& +4 x_{a}^{2} B_{11} B_{2_{a} 2_{a}} \rho_{1} \rho_{2}+4 x_{b}^{2} B_{11} B_{2_{b} 2_{b}} \rho_{1} \rho_{2}+4 x_{a} x_{b} B_{2_{a} 2_{b}} \rho_{2} \\
& +8 x_{a} x_{b} B_{11} B_{2_{a} 2_{b}} \rho_{1} \rho_{2}-8 x_{a} x_{b} B_{12_{b}} B_{12_{a}} \rho_{1} \rho_{2}+1
\end{aligned}
$$

It is clear that Eqs. (2.66) and (2.71) are different. Using the effective virial coefficients to determine stability of the mixture possibly results in deviations. 


\subsubsection{Critical points}

In a binary mixture, the critical point is a stable point which lies on the stability limit (spinodal) (Heidemann and Khalil, 1980) and where the phase boundary and spinodal coincide. In mixtures of more components these become plait points. Critical points and plait points are in general concentrations at which two phases in equilibrium become indistinguishable (Heidemann, 1994).

There are two criteria that have to be used to find critical points. The first one is $\operatorname{det}\left(M_{1}\right)=0$, which is the equation for the spinodal. The other criterion is based on the fact that at the critical point, the third derivative of the free energy should also be zero. For a multicomponent system, this criterion can be reformulated using Legendre transforms as $\operatorname{det}\left(M_{2}\right)=0$ (Beegle et al., 1974; Reid and Beegle, 1977), where:

$$
M_{2}=\left[\begin{array}{ccc}
\frac{\partial \mu_{1}}{\partial N_{1}} & \cdots & \frac{\partial \mu_{n}}{\partial N_{n}} \\
\vdots & \ddots & \vdots \\
\frac{\partial M_{1}}{\partial N_{1}} & \cdots & \frac{\partial M_{1}}{\partial N_{n}}
\end{array}\right]
$$

Matrix $M_{2}$ is matrix $M_{1}$ with one of the rows replaced by the partial derivatives of the determinant of matrix $M_{1}$. Note: it does not matter which row of the matrix is replaced.

For a monodisperse binary mixture, this results in the following two matrices for the critical point:

$$
M_{1}=\left[\begin{array}{cc}
2 B_{11}+\frac{1}{\rho_{1}} & 2 B_{12} \\
2 B_{12} & 2 B_{22}+\frac{1}{\rho_{2}}
\end{array}\right]
$$

and

$$
M_{2}=\left[\begin{array}{cc}
2 B_{11}+\frac{1}{\rho_{1}} & 2 B_{12} \\
-\frac{2 B_{22} \rho_{2}+1}{\rho_{1}^{2} \rho_{2}} & -\frac{2 B_{11} \rho_{1}+1}{\rho_{1} \rho_{2}^{2}}
\end{array}\right]
$$

The set of equations that needs be solved for the critical point is:

$$
\left\{\begin{array}{l}
\operatorname{det}\left(M_{1}\right)=1+2 B_{11} \rho_{1}+2 B_{22} \rho_{2}-4 B_{12}^{2} \rho_{1} \rho_{2}+4 B_{11} B_{22} \rho_{1} \rho_{2}=0 \\
\operatorname{det}\left(M_{2}\right)=\left(2 B_{12} \rho_{2}\right)\left(1+2 B_{22} \rho_{2}\right)-\left(1+2 B_{11} \rho_{1}\right)^{2}=0
\end{array}\right.
$$

For the earlier considered polydisperse mixture containing the two sub-components ( $a$ and $b, n=3$ ) we obtain: 


$$
M_{2}=\left[\begin{array}{ccc}
2 B_{11}+\frac{1}{\rho_{1}} & 2 B_{12_{a}} & 2 B_{12_{b}} \\
2 B_{12_{a}} & 2 B_{2_{a} 2_{a}}+\frac{1}{\rho_{2_{a}}} & 2 B_{2_{a} 2_{b}} \\
P_{1} & P_{2} & P_{3}
\end{array}\right]
$$

with

$$
\begin{aligned}
& P_{1}=-\frac{\left(\begin{array}{c}
-4 x_{a} x_{b} B_{2_{a} 2_{b}}^{2} \rho_{2}^{2}+2 x_{a} B_{2_{a} 2_{a}} \rho_{2} \\
+2 x_{b} B_{2_{b} 2_{b}} \rho_{2}+4 x_{a} x_{b} B_{2_{a} 2_{a}} B_{2_{b} 2_{b}} \rho_{2}^{2}+1
\end{array}\right)}{x_{a} x_{b} \rho_{1}^{2} \rho_{2}^{2}} \\
& P_{2}=-\frac{\left(\begin{array}{c}
-4 x_{b} B_{12_{b}}^{2} \rho_{1} \rho_{2}+2 B_{11} \rho_{1}+2 x_{b} B_{2_{b} 2_{b}} \rho_{2} \\
+4 x_{b} B_{11} B_{2_{b} 2_{b}} \rho_{1} \rho_{2}+1
\end{array}\right)}{\left.x_{a}^{2} x_{b} \rho_{1} \rho_{2}^{3}\right)} \\
& P_{3}=-\frac{\left(\begin{array}{c}
-4 x_{a} B_{12_{a}}^{2} \rho_{1} \rho_{2}+2 B_{11} \rho_{1}+2 x_{a} B_{2_{a} 2_{a}} \rho_{2} \\
+4 x_{a} B_{11} B_{2_{a} 2_{a}} \rho_{1} \rho_{2}+1
\end{array}\right)}{x_{a} x_{b}^{2} \rho_{1} \rho_{2}^{3}}
\end{aligned}
$$

In general, $P_{i}$ can be found using the following equation:

$$
P_{i}=-\frac{1}{\rho_{i}^{2}} M_{1,(i i)}
$$

in which, $M_{1,(i i)}$ is the minor of matrix $M_{1}$ at the $i^{\text {th }}$-row and $i^{t h}$-column.

Combining $\operatorname{det}\left(M_{1}\right)$ and $\operatorname{det}\left(M_{2}\right)$ results in the following set of equations:

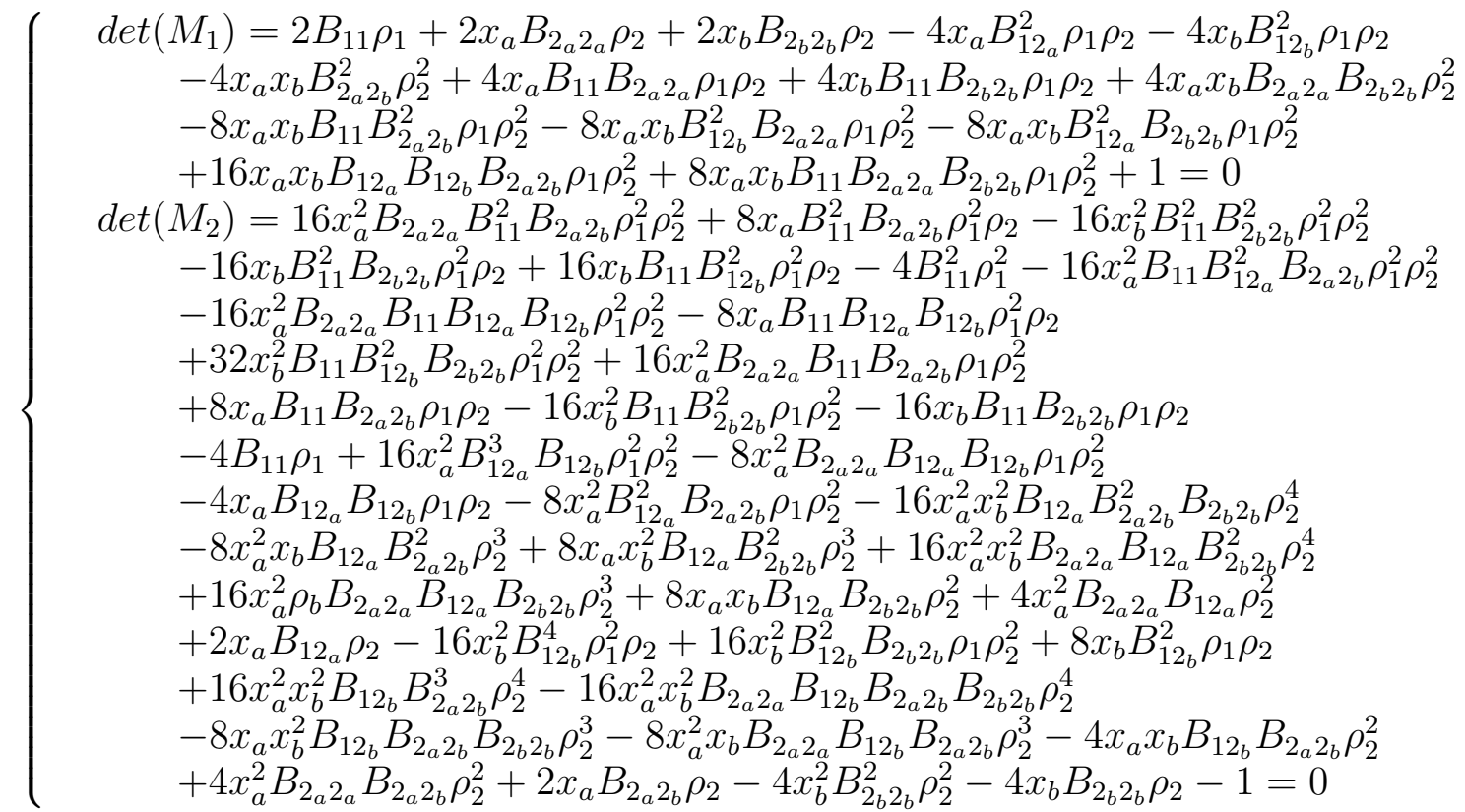


Since in this set of equations there are more higher order terms present, it is possible that this results in multiple plait points, depending on the concentration of each of the components in the mixture. Care should be taken that the solutions of the set of equations are concentrations are at the limit of stability, this can be done by checking the eigenvalues of the stability matrix.

If we use the effective virial coefficients for this mixture as defined in Section 2.2.4, we obtain:

$$
M_{2_{e f f}}=\left[\begin{array}{cc}
2 B_{11_{e f f}}+\frac{1}{\rho_{1}} & 2 B_{12_{e f f}} \\
-\frac{2 B_{22_{e} f f} \rho_{2}+1}{\rho_{1}^{2} \rho_{2}} & -\frac{2 B_{11_{e} f f} \rho_{1}+1}{\rho_{1} \rho_{2}^{2}}
\end{array}\right]
$$

The determinant of this matrix becomes:

$$
\begin{aligned}
\operatorname{det}\left(M_{2_{e f f}}\right)= & -4 B_{11_{e} f f}^{2} \rho_{1}^{2}-4 B_{11_{e} f f} \rho_{1}+4 B_{12_{e} f f} B_{22_{e} f f} \rho_{2}^{2}+2 B_{12_{e} f f} \rho_{2}-1 \\
= & -4 B_{11}^{2} \rho_{1}^{2}-4 B_{11} \rho_{1}+4\left(x_{a} B_{12_{a}}+x_{b} B_{12_{b}}\right) \times\left(x_{a}^{2} B_{2_{a} 2_{b}}+2 x_{a} x_{b} B_{2_{a} 2_{b}}\right. \\
& \left.+x_{b}^{2} B_{2_{b} 2_{b}}\right) \rho_{2}^{2}+2\left(x_{a} B_{12_{a}}+x_{b} B 12_{b}\right) \rho_{2}-1 \\
= & -4 B_{11}^{2} \rho_{1}^{2}-4 B_{11} \rho_{1}+4 x_{a}^{3} B_{12_{a}} B_{2_{a} 2_{a}} \rho_{2}^{2}+8 x_{a}^{2} x_{b} B_{12_{a}} B_{2_{a} 2_{b}} \rho_{2}^{2} \\
& +4 x_{a} x_{b}^{2} B_{12_{a}} B_{2_{b} 2_{b}} \rho_{2}^{2}+4 x_{a}^{2} x_{b} B_{12_{b}} B_{2_{a} 2_{a}} \rho_{2}^{2}+8 x_{a} x_{b}^{2} B_{12_{b}} B_{2_{a} 2_{b}} \rho_{2}^{2} \\
& +4 x_{b}^{3} B_{12_{b}} B_{2_{b} 2_{b}} \rho_{2}^{2}+2 x_{a} B_{12_{a}} \rho_{2}+2 x_{b} B_{12_{b}} \rho_{2}-1
\end{aligned}
$$

Which results in the following system of equations for the critical point:

$$
\left\{\begin{array}{c}
\operatorname{det}\left(M_{1_{e} f f}\right)=2 B_{11} \rho_{1}+2 x_{a}^{2} B_{2_{a} 2_{a}} \rho_{2}+2 x_{b}^{2} B_{2_{b} 2_{b}} \rho_{2}-4 x_{a}^{2} B_{12_{a}}^{2} \rho_{1} \rho_{2}-4 x_{b}^{2} B_{12_{b}}^{2} \rho_{1} \rho_{2} \\
\quad+4 x_{a}^{2} B_{11} B_{2_{a} 2_{a}} \rho_{1} \rho_{2}+4 x_{b}^{2} B_{11} B_{2_{b} 2_{b}} \rho_{1} \rho_{2}+4 x_{a} x_{b} B_{2_{a} 2_{b}} \rho_{2}+8 x_{a} x_{b} B_{11} B_{2_{a} 2_{b}} \rho_{1} \rho_{2} \\
\quad-8 x_{a} x_{b} B_{12_{b}} B_{12_{a}} \rho_{1} \rho_{2}+1=0 \\
\operatorname{det}\left(M_{2_{e} f f}\right)=-4 B_{11}^{2} \rho_{1}^{2}-4 B_{11} \rho_{1}+4 x_{a}^{3} B_{12_{a}} B_{2_{a} 2_{a}} \rho_{2}^{2}+8 x_{a}^{2} x_{b} B_{12_{a}} B_{2_{a} 2_{b}} \rho_{2}^{2} \\
\quad+4 x_{a} x_{b}^{2} B_{12_{a}} B_{2_{b} 2_{b}} \rho_{2}^{2}+4 x_{a}^{2} x_{b} B_{12_{b}} B_{2_{a} a_{a}} \rho_{2}^{2}+8 x_{a} x_{b}^{2} B_{12_{b}} B_{2_{a} 2_{b}} \rho_{2}^{2} \\
+4 x_{b}^{3} B_{12_{b}} B_{2_{b} 2_{b}} \rho_{2}^{2}+2 x_{a} B_{12_{a}} \rho_{2}+2 x_{b} B_{12_{b}} \rho_{2}-1=0
\end{array}\right.
$$

Also for the third derivative of the Helmholtz free energy, we see that reducing the polydispersity by using the effective virial coefficients, results in fewer terms in the equation and possible deviations in determining the critical point.

\subsubsection{Phase boundary}

When a mixture becomes unstable and phase separates into two or more phases, the chemical potential of each component and the osmotic pressure is the same in all phases Hill (1986). 


$$
\left\{\begin{array}{c}
\beta \Pi^{I}=\beta \Pi^{I I}=\cdots \\
\beta \mu_{1}^{I}=\beta \mu_{1}^{I I}=\cdots \\
\vdots \\
\beta \mu_{n}^{I}=\beta \mu_{n}^{I I}=\cdots
\end{array}\right.
$$

where the phases are denoted by $I, I I, \ldots$.

For a system that separates into two phases, we obtain, using Eqs. (2.56) and (2.60), the following set of equations for the general case of $n$ components:

$$
\left\{\begin{array}{c}
\rho_{1}^{I}+\cdots+\rho_{n}^{I}+\sum_{i}^{n} \sum_{j}^{n} B_{i j} \rho_{i}^{I} \rho_{j}^{I} \\
= \\
\rho_{1}^{I I}+\cdots+\rho_{n}^{I I}+\sum_{i}^{n} \sum_{j}^{n} B_{i j} \rho_{i}^{I I} \rho_{j}^{I I} \\
\ln \left(\rho_{1}^{I}\right)+2\left(\sum_{j}^{n} B_{1 j} \rho_{j}^{I}\right)=\ln \left(\rho_{1}^{I I}\right)+2\left(\sum_{j}^{n} B_{1 j} \rho_{j}^{I I}\right) \\
\vdots \\
\ln \left(\rho_{n}^{I}\right)+2\left(\sum_{j}^{n} B_{n j} \rho_{j}^{I}\right)=\ln \left(\rho_{n}^{I I}\right)+2\left(\sum_{j}^{n} B_{n j} \rho_{j}^{I I}\right)
\end{array}\right.
$$

This set of equations has $2 \times n$ unknowns and $n+1$ equations. The set of equations can be solved by fixing one of the concentrations for one phase and the ratio of the concentrations of the other components for the same phase. To solve this set of equations in order to find the concentration of each component in each phase, without fixing any of the concentrations, we need therefore an extra set of equations.

This extra set of equations stems from the fact that during phase separation no particles are lost and no new particles are created. The total number of components in the system is therefore given by:

$$
N=\sum_{i}^{n} N_{i}^{I}+\sum_{i}^{n} N_{i}^{I I}
$$

Also the total volume, $V$, of the system does not change. With the total volume of the system given by:

$$
V=V^{I}+V^{I I}
$$

the concentrations of each component in each phase are thus given by: 


$$
\begin{array}{ccc}
\rho_{1}^{I}=\frac{N_{1}^{I}}{V^{I}} & \cdots & \rho_{n}^{I}=\frac{N_{n}^{I}}{V^{I}} \\
\rho_{1}^{I I}=\frac{N_{1}^{I I}}{V^{I I}} & \cdots & \rho_{n}^{I I}=\frac{N_{n}^{I I}}{V^{I I}}
\end{array}
$$

The total number of compounds in the system can be found using:

$$
\rho=\sum_{i}^{n} \rho_{i}=\frac{\sum_{i}^{n} N_{i}}{V}=\frac{\sum_{i}^{n} N_{i}^{I}+\sum_{i}^{n} N_{i}^{I I}}{V^{I}+V^{I I}}
$$

which can be rewritten to:

$$
\begin{aligned}
\rho & =\sum_{i}^{n} \frac{N_{i}^{I}}{V^{I}+V^{I I}}+\sum_{i}^{n} \frac{N_{i}^{I I}}{V^{I}+V^{I I}} \\
& =\frac{V^{I}}{V^{I}+V^{I I}} \sum_{i}^{n} \frac{N_{i}^{I}}{V^{I}}+\frac{V^{I I}}{V^{I}+V^{I I}} \sum_{i}^{n} \frac{N_{i}^{I I}}{V^{I}+V^{I I}} \\
& =\alpha \sum_{i}^{n} \rho_{i}^{I}+(1-\alpha) \sum_{i}^{n} \rho_{i}^{I I}=\sum_{i}^{n} \rho_{i}
\end{aligned}
$$

with

$$
\alpha=\frac{V^{I}}{V^{I}+V^{I I}}
$$

This results in an extra set of $n$ equations and one more unknown $(\alpha)$. The complete set of equations to solve for the binodal then becomes:

$$
\left\{\begin{aligned}
\rho_{1}^{I}+\cdots+\rho_{n}^{I}+\sum_{i}^{n} \sum_{j}^{n} B_{i j} \rho_{i}^{I} \rho_{j}^{I} & \\
& = \\
\rho_{1}^{I I}+\cdots+\rho_{n}^{I I}+ & \sum_{i}^{n} \sum_{j}^{n} B_{i j} \rho_{i}^{I I} \rho_{j}^{I I} \\
\ln \left(\rho_{1}^{I}\right)+2\left(\sum_{j}^{n} B_{1 j} \rho_{j}^{I}\right) & =\ln \left(\rho_{1}^{I I}\right)+2\left(\sum_{j}^{n} B_{1 j} \rho_{j}^{I I}\right) \\
\vdots & \\
\ln \left(\rho_{n}^{I}\right)+2\left(\sum_{j}^{n} B_{n j} \rho_{j}^{I}\right) & =\ln \left(\rho_{n}^{I I}\right)+2\left(\sum_{j}^{n} B_{n j} \rho_{j}^{I I}\right) \\
\rho_{1} & =\alpha \rho_{1}^{I}+(1-\alpha) \rho_{1}^{I I} \\
\vdots & \\
\rho_{n} & =\alpha \rho_{n}^{I}+(1-\alpha) \rho_{n}^{I I}
\end{aligned}\right.
$$


Systems for which there are more than two distinguishable components $(n>2)$ can theoretically have more than two coexisting phases, according to the Gibbs phase rule. With an increasing number of phases, the set of equations to solve increases as well. The number of equations needed to solve for an arbitrary number of $f$ phases is: $f \times n+f-1$. The set of equations then becomes:

$$
\begin{aligned}
& \rho_{1}^{I}+\cdots+\rho_{n}^{I}+\sum_{i}^{n} \sum_{j}^{n} B_{i j} \rho_{i}^{I} \rho_{j}^{I}=\rho_{1}^{I I}+\cdots+\rho_{n}^{I I}+\sum_{i}^{n} \sum_{j}^{n} B_{i j} \rho_{i}^{I I} \rho_{j}^{I I} \\
& \rho_{1}^{f-1}+\cdots+\rho_{n}^{f-1}+\sum_{i}^{n} \sum_{j}^{n} B_{i j} \rho_{i}^{f-1} \rho_{j}^{f-1}=\rho_{1}^{f}+\cdots+\rho_{n}^{f}+\sum_{i}^{n} \sum_{j}^{n} B_{i j} \rho_{i}^{f} \rho_{j}^{f} \\
& \ln \left(\rho_{1}^{I}\right)+2\left(\sum_{j}^{n} B_{1 j} \rho_{j}^{I}\right)=\ln \left(\rho_{1}^{I I}\right)+2\left(\sum_{j}^{n} B_{1 j} \rho_{j}^{I I}\right) \\
& \ln \left(\rho_{1}^{f-1}\right)+2\left(\sum_{j}^{n} B_{1 j} \rho_{j}^{f-1}\right)=\ln \left(\rho_{1}^{f}\right)+2\left(\sum_{j}^{n} B_{1 j} \rho_{j}^{f}\right) \\
& \ln \left(\rho_{n}^{I}\right)+2\left(\sum_{j}^{n} B_{n j} \rho_{j}^{I}\right)=\ln \left(\rho_{n}^{I I}\right)+2\left(\sum_{j}^{n} B_{n j} \rho_{j}^{I I}\right) \\
& \ln \left(\rho_{n}^{f-1}\right)+2\left(\sum_{j}^{n} B_{n j} \rho_{j}^{f-1}\right)=\ln \left(\rho_{n}^{f}\right)+2\left(\sum_{j}^{n} B_{n j} \rho_{j}^{f}\right) \\
& \rho_{1}=\alpha_{1} \rho_{1}^{I}+\alpha_{2} \rho_{1}^{I I}+\cdots+\left(1-\sum_{i}^{f-1} \alpha_{i}\right) \rho_{1}^{f} \\
& \rho_{n}=\alpha_{1} \rho_{n}^{I}+\alpha_{2} \rho_{n}^{I I}+\cdots+\left(1-\sum_{i}^{f-1} \alpha_{i}\right) \rho_{n}^{f}
\end{aligned}
$$

with

$$
\alpha_{1}=\frac{V^{I}}{\sum_{i}^{f} V^{i}} \quad \cdots \quad \alpha_{f-1}=\frac{V^{f-1}}{\sum_{i}^{f} V^{i}}
$$

Now that we have the general equations for the phaseboundary, let us investigate the set of equations we need to solve for the mixtures we defined earlier. For a monodisperse 
binary mixture the set of equations to solve for the phase boundary is given by:

$$
\left\{\begin{aligned}
\rho_{1}^{I}+\rho_{2}^{I}+B_{11} \rho_{1}^{I^{2}}+2 B_{12} \rho_{1}^{I} \rho_{2}^{I}+B_{22} \rho_{2}^{I^{2}} & =\rho_{1}^{I I}+\rho_{2}^{I I}+B_{11} \rho_{1}^{I I^{2}}+2 B_{12} \rho_{1}^{I I} \rho_{2}^{I I}+B_{22} \rho_{2}^{I I^{2}} \\
\ln \left(\rho_{1}^{I}\right)+2 B_{11} \rho_{1}^{I}+2 B_{12} \rho_{2}^{I} & =\ln \left(\rho_{1}^{I I}\right)+2 B_{11} \rho_{1}^{I I}+2 B_{12} \rho_{2}^{I I} \\
\ln \left(\rho_{2}^{I}\right)+2 B_{12} \rho_{1}^{I}+2 B_{22} \rho_{2}^{I} & =\ln \left(\rho_{2}^{I I}\right)+2 B_{12} \rho_{1}^{I I}+2 B_{22} \rho_{2}^{I I} \\
\rho_{1} & =\alpha \rho_{1}^{I}+(1-\alpha) \rho_{1}^{I I} \\
\rho_{2} & =\alpha \rho_{2}^{I}+(1-\alpha) \rho_{2}^{I I}
\end{aligned}\right.
$$

For the polydisperse binary mixture we considered earlier (with two sub-components $a$ and $b, n=3)$ this set of equations becomes:

$$
\left\{\begin{array}{c}
\rho_{1}^{I}+\rho_{2_{a}}^{I}+\rho_{2_{b}}^{I}+B_{11} \rho_{1}^{I^{2}}+2 B_{12_{a}} \rho_{1}^{I} \rho_{2_{a}}^{I}+2 B_{12_{b}} \rho_{1}^{I} \rho_{2_{b}}^{I} \\
+B_{2_{a} 2_{a}} \rho_{2_{a}}^{I^{2}}+2 B_{2_{a} 2_{b}} \rho_{2_{a}}^{I} \rho_{2_{a}}^{I}+B_{2_{b} 2_{b}} \rho_{2_{b}}^{I^{2}}=\rho_{1}^{I I}+\rho_{2_{a}}^{I I}+\rho_{2_{b}}^{I I} \\
+B_{11} \rho_{1}^{I I^{2}}+2 B_{12_{a}} \rho_{1}^{I I} \rho_{2_{a}}^{I I}+2 B_{12_{b}} \rho_{1}^{I I} \rho_{2_{b}}^{I I}+B_{2_{a} 2_{a}} \rho_{2_{a}}^{I I^{2}}+2 B_{2_{a} 2_{b}} \rho_{2_{a}}^{I I} \rho_{2_{b}}^{I I}+B_{2_{b} 2_{b}} \rho_{2_{b}}^{I I^{2}} \\
\ln \left(\rho_{1}^{I}\right)+2 B_{11} \rho_{1}^{I}+2 B_{12_{a}} \rho_{2_{a}}^{I}+2 B_{12_{b}} \rho_{2_{b}}^{I} \\
=\ln \left(\rho_{1}^{I I}\right)+2 B_{11} \rho_{1}^{I I}+2 B_{12_{a}} \rho_{2_{a}}^{I I}+2 B_{12_{b}} \rho_{2_{b}}^{I I} \\
\ln \left(\rho_{2_{a}}^{I}\right)+2 B_{12_{a}} \rho_{1}^{I}+2 B_{2_{a} 2_{a}} \rho_{2_{a}}^{I}+2 B_{2_{a} 2_{b}} \rho_{2_{b}}^{I} \\
=\ln \left(\rho_{2_{a}}^{I I}\right)+2 B_{12_{a}} \rho_{1}^{I I}+2 B_{2_{a} 2_{a}} \rho_{2_{a}}^{I I}+2 B_{2_{a} 2_{b}} \rho_{2_{b}}^{I I} \\
\ln \left(\rho_{2_{b}}^{I}\right)+2 B_{12_{b}} \rho_{1}^{I}+2 B_{2_{a} 2_{b}} \rho_{2_{a}}^{I}+2 B_{2_{b} 2_{b}} \rho_{2_{b}}^{I} \\
=\ln \left(\rho_{2_{b}}^{I I}\right)+2 B_{12_{b}} \rho_{1}^{I I}+2 B_{2_{a} 2_{b}} \rho_{2_{a}}^{I I}+2 B_{2_{b} 2_{b}} \rho_{2_{b}}^{I I} \\
\rho_{1}=\alpha \rho_{1}^{I}+(1-\alpha) \rho_{1}^{I I} \\
\rho_{2_{a}}=\alpha \rho_{2_{a}}^{I}+(1-\alpha) \rho_{2_{a}}^{I I} \\
\rho_{2_{b}}=\alpha \rho_{2_{b}}^{I}+(1-\alpha) \rho_{2_{b}}^{I I}
\end{array}\right.
$$

Note that the ratio between $\rho_{2_{a}}^{I}$ and $\rho_{2_{b}}^{I}$ is not necessarily the same as the ratio between $\rho_{2_{a}}^{I I}$ and $\rho_{2_{b}}^{I I}$, since fractionation between the components can occur.

Using the effective virial coefficients we obtain:

$$
\left\{\begin{aligned}
\rho_{1}^{I}+\rho_{2}^{I}+B_{11_{e f f}} \rho_{1}^{I^{2}}+2 B_{12_{e f f}} \rho_{1}^{I} \rho_{2}^{I} & +B_{22_{e f f}} \rho_{2}^{I^{2}} \\
= & \rho_{1}^{I I}+\rho_{2}^{I I}+B_{11_{e f f}} \rho_{1}^{I I^{2}}+2 B_{12_{e f f}} \rho_{1}^{I I} \rho_{2}^{I I}+B_{22_{e f f}} \rho_{2}^{I I^{2}} \\
\ln \left(\rho_{1}^{I}\right)+2 B_{11_{e f f}} \rho_{1}^{I}+2 B_{12_{e f f}} \rho_{2}^{I} & =\ln \left(\rho_{1}^{I I}\right)+2 B_{11_{e f f}} \rho_{1}^{I I}+2 B_{12_{e f f}} \rho_{2}^{I I} \\
\ln \left(\rho_{2}^{I}\right)+2 B_{12_{e f f}} \rho_{1}^{I}+2 B_{22_{e f f}} \rho_{2}^{I} & =\ln \left(\rho_{2}^{I I}\right)+2 B_{12_{e f f}} \rho_{1}^{I I}+2 B_{22_{e f f}} \rho_{2}^{I I} \\
\rho_{1} & =\alpha \rho_{1}^{I}+(1-\alpha) \rho_{1}^{I I} \\
\rho_{2} & =\alpha \rho_{2}^{I}+(1-\alpha) \rho_{2}^{I I}
\end{aligned}\right.
$$


It is clear from the equations, that when using the effective virial coefficients for calculating the phase boundary all information about the (changes in) distribution of the polydisperse component becomes untraceable.

\subsection{Results and discussion}

In this work we calculated the liquid-liquid phase diagram for a variety of binary additive mixtures of a small hard sphere $A$ and a larger hard sphere $B$ with a size ratio $q=$ $\sigma_{A} / \sigma_{B}=1 / 10$. We started by calculating the phase diagram of this monodisperse mixture (Figure 2.2) and gradually introduced polydispersity into the composition of component $B$ (Figures 2.3 to 2.6 ). Component $B$ is characterized by a degree in polydispersity $(P D)$, defined by:

$$
P D=\frac{\sqrt{\sum\left(\sigma_{B_{i}}-\sigma_{B}\right)^{2} \times N_{B_{i}} / N_{B}}}{\sigma_{B}} \times 100
$$

For all particles, the concentrations are expressed as a dimensionless parameter according to $\eta=\frac{\pi \rho \sigma^{3}}{6}$. We calculated the critical point, the phase separation boundary, and the spinodal of the various mixtures. Next to that, we also investigated the composition of the child phases, volume ratio between the phases $(\alpha)$, and the fractionation of the polydisperse component $B$ for a specific parent mixture.

In order to be able to compare our results with the monodisperse case we first refer to Figure 2.2, where we see that the binary mixture phase separates at very low volume fractions of component $A\left(\eta_{A_{\text {crit }}}=0.007\right)$ and significantly higher concentrations of component $B\left(\eta_{B_{c r i t}}=0.267\right)$. This high asymmetry in the position of the critical point and phase boundary has also previously been reported by Hopkins and Schmidt (2010). Note that even though the volume fraction of the smaller spheres are very low, their number concentration is significantly higher than the large spheres.

\subsubsection{Polydisperse mixtures with 2 sub-components}

In Figure 2.3 we show the phase diagram for the case of a slight polydispersity in component $B$. Component $B$ consists of two sub-components and has a $P D=4.00$. These components are additive spheres in two sizes (both present in the same amount), with the number average size of the mixture equal to that of the size of the monodisperse mixture of Figure 2.2. The mixture therefore consist of three components. We calculated the phase diagram using both the simplified $2 \times 2$ effective virial coefficient matrix described in the theory (we refer to this as the effective mixture) and the full $3 \times 3$ virial coefficient matrix (to which we 


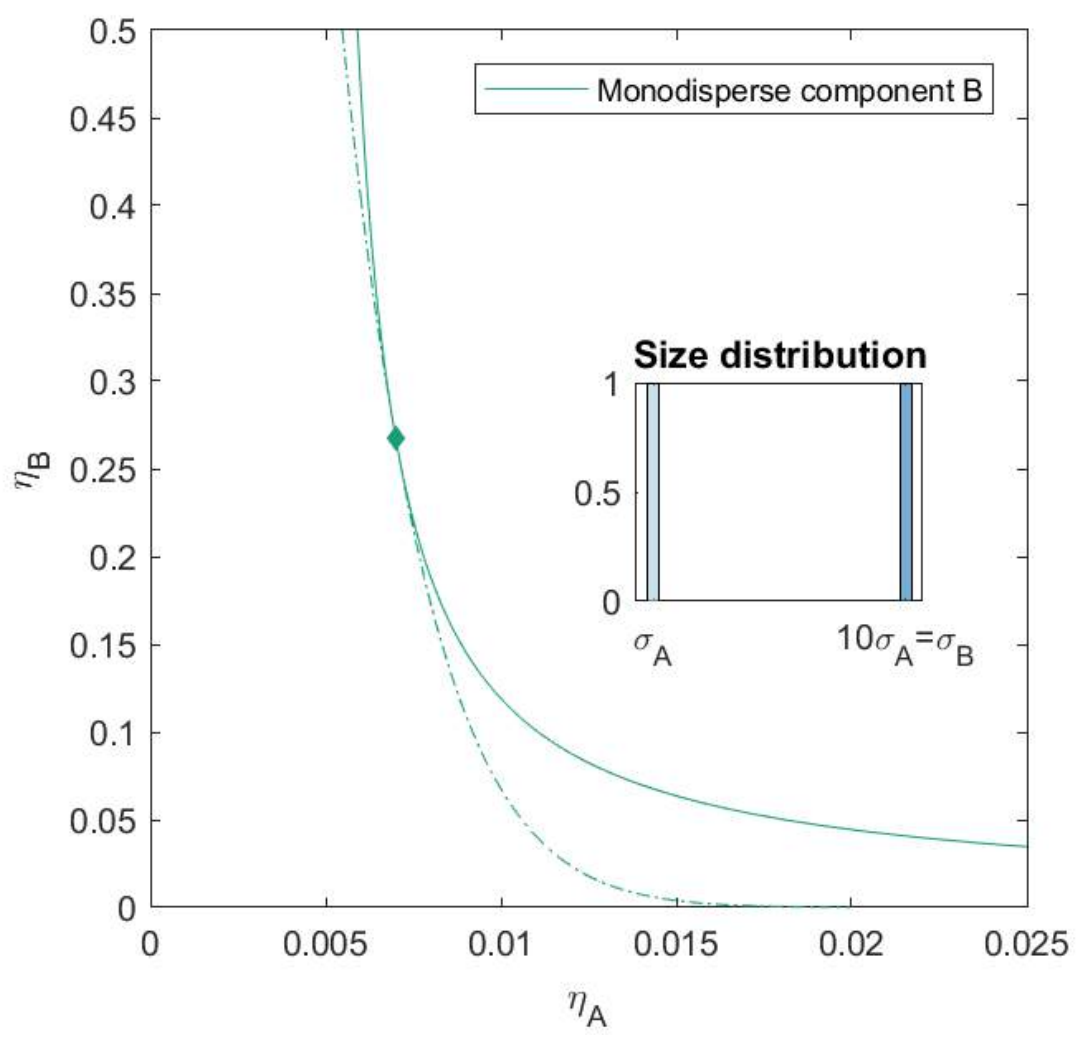

Figure 2.2: Phase diagram for monodisperse binary (component $A$ and $B$ ) additive hard sphere mixture with size ratio $q=\sigma_{A} / \sigma_{B}=1 / 10$, plotted as a function of the partial packing fractions, $\eta_{A}$ and $\eta_{B}$. The spinodal (solid line) and binodal (dashed line) meet each other at the critical point (diamond).

refer as the polydisperse mixture). The difference between the phase boundary, spinodal and critical point of the monodisperse mixture and the effective mixture is negligible. We see however that the introduction of the polydispersity causes the critical point to shift to a higher volume fraction of component $B\left(\eta_{A_{\text {crit }}}=0.007, \eta_{B_{\text {crit }}}=0.280\right)$ and that especially at lower volume fraction of component $B$ the phase separation boundary shifts towards slightly lower packing fractions.

For Figure 2.4 we increased the size difference between the smaller and the larger spheres for component $B$, with the standard deviation twice the standard deviation of the spheres in Figure 2.3 and the $P D=8.00$. The patterns we saw in Figure 2.3 are more pronounced for this mixture: the increase in size difference causes the critical point to shift to higher packing fractions for component $B$ and lower packing fractions for component $A$ $\left(\eta_{A_{\text {crit }}}=0.006, \eta_{B_{\text {crit }}}=0.315\right)$. The phase boundary, i.e. the binodal, shifts to significantly lower packing fractions, especially at lower volume concentrations of component $B$. For this mixture we see also that the spinodal of the polydisperse mixture shifts towards lower 


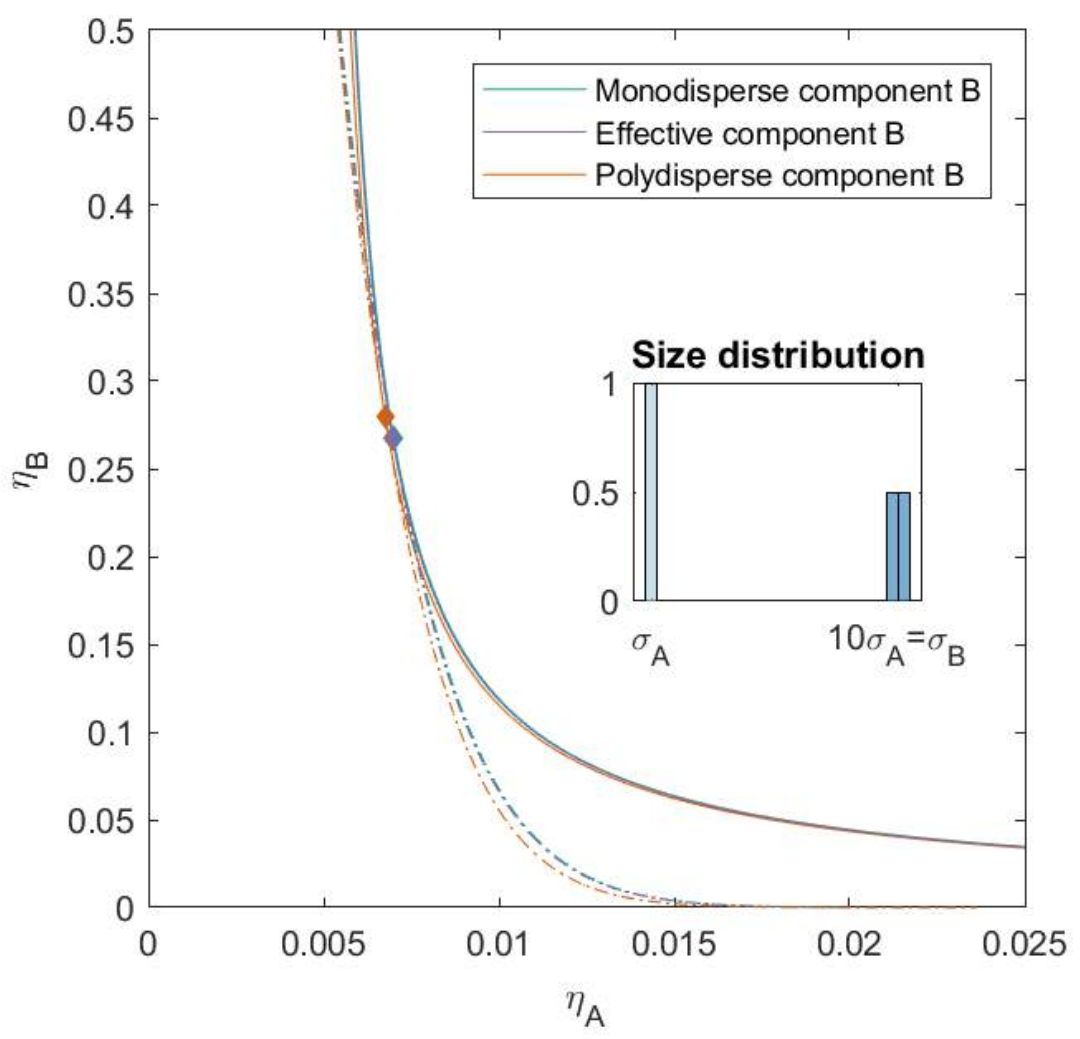

Figure 2.3: Phase diagram for binary (component $A$ and $B$ ) additive hard sphere mixture with size ratio $q=\sigma_{A} / \sigma_{B}=1 / 10$, component $A$ is monodisperse, component $B$ is polydisperse (see size distribution, $(P D=4.00))$, with a number average size 10 times the size of component $A$, plotted as a function of the partial packing fractions, $\eta_{A}$ and $\eta_{B}$. The spinodal (solid line) and binodal (dashed line) meet each other at the critical point (diamond).

packing fractions of $A$. There is a slight difference between the positions of the binodal, spinodal and critical point of the monodisperse mixture and effective mixture.

In Figure 2.5 we introduced skewness in the size distribution of component $B$. For the first two mixtures (Figures 2.5a and 2.5b) the ratio between the bigger and the smaller sub-component was $25 / 75$. The polydispersity for both mixtures is the same, $P D=6.93$. For the other two mixtures (Figures 2.5c and 2.5d), the ratio between the bigger and the smaller sub-component was more extreme, namely $90 / 10$, with $P D=4.80$. For all these mixtures, we find that the critical point shifts towards higher packing fraction of component $B\left(\left(\eta_{A_{\text {crit }}}=0.006, \eta_{B_{\text {crit }}} 0.290\right)\right.$ for Figure 2.5a, $\left(\eta_{A_{\text {crit }}}=0.006, \eta_{B_{\text {crit }}} 0.326\right)$ for Figure 2.5b, $\left(\eta_{A_{\text {crit }}}=0.007, \eta_{B_{\text {crit }}} 0.276\right)$ for Figure 2.5c, and $\left(\eta_{A_{\text {crit }}}=0.007, \eta_{B_{\text {crit }}} 0.304\right)$ for Figure 2.5d).

Next to this shift in critical point, we also see that the spinodal shifts towards lower packing fractions of component $A$, and that the binodal shifts towards lower packing fraction for lower concentrations of component $B$, although there is a difference in the amount of 


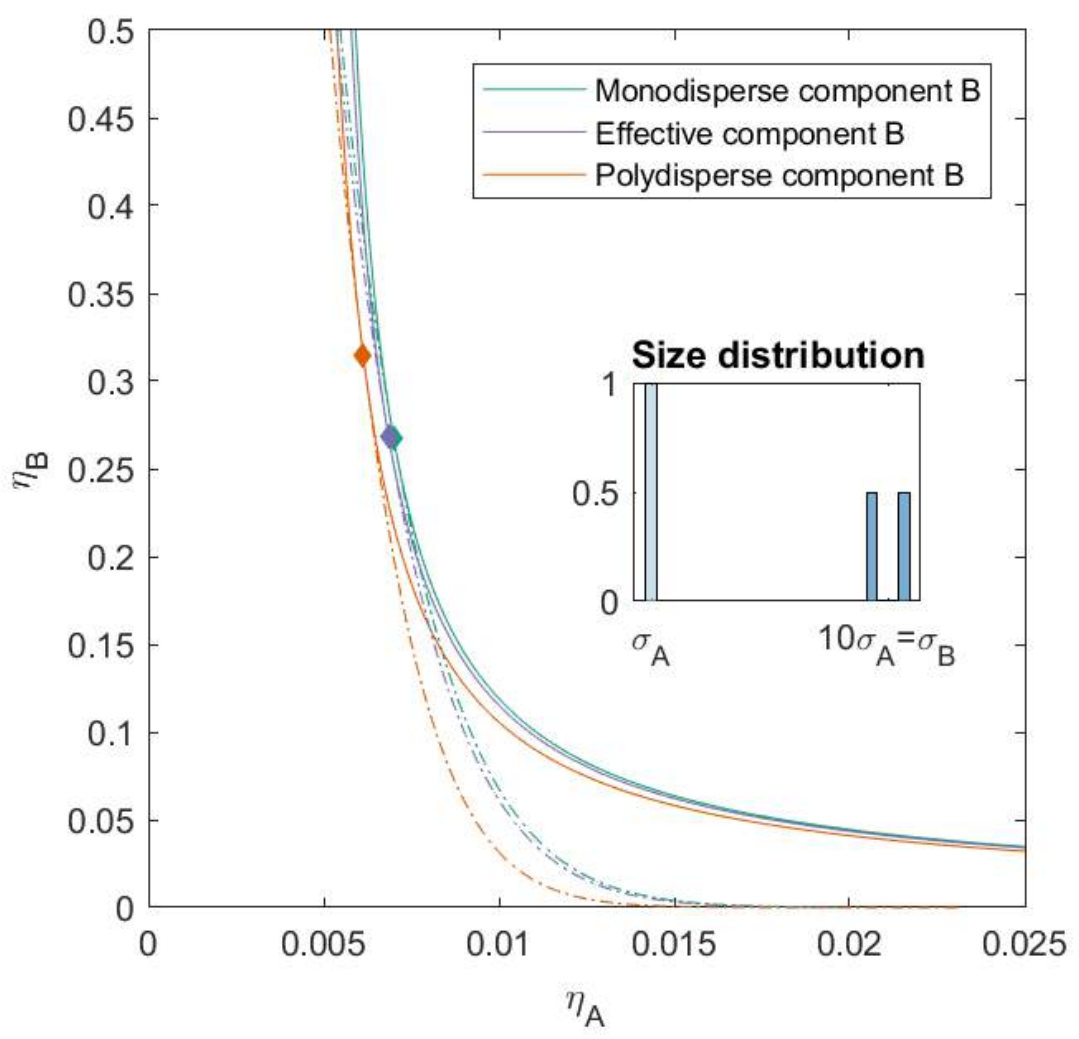

Figure 2.4: Phase diagram for binary (component $A$ and $B$ ) additive hard sphere mixture with size ratio $q=\sigma_{A} / \sigma_{B}=1 / 10$, component $A$ is monodisperse, component $B$ is polydisperse (see size distribution, SD twice of mixture in Figure 2.3), with a number average size 10 times the size of component $A$, plotted as a function of the partial packing fractions, $\eta_{A}$ and $\eta_{B}$. The spinodal (solid line) and binodal (dashed line) meet each other at the critical point (diamond).

shift. We kept the number average size of the spheres for component $B$ the same for all mixtures, meaning that the largest spheres in the mixture in Figures 2.5b and 2.5d are larger than the largest spheres in the mixture in Figures 2.5a and 2.5c. From this we can conclude that the larger spheres, even though they are smaller in number, have a higher impact on the concentration of the critical point and the position of the phase boundary.

Looking at the different mixtures (Figures 2.3 to 2.5 ) we can conclude that polydispersity shifts the critical point to higher packing fraction for component $B$ and lower packing fractions of component $A$ compared to a monodisperse mixture with the same average sizes. The phase boundary shifts to lower packing fractions at lower concentrations of component $B$. The shift is dependent on the size distribution and then in a large part on the size and the concentration of the largest particle in the mixture. 


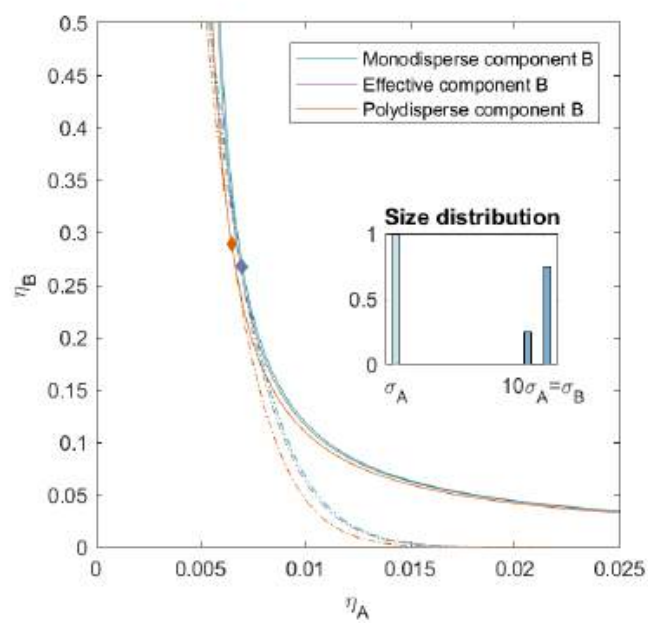

(a) Large amount of larger spheres

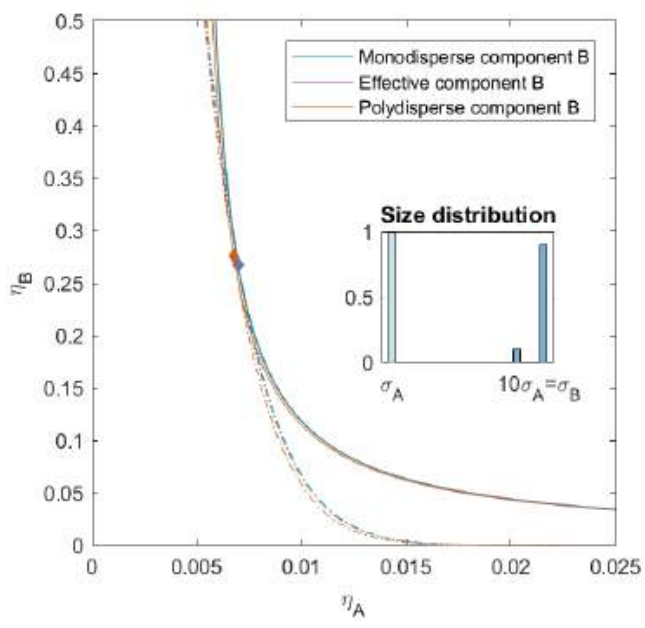

(c) Large amount of larger spheres

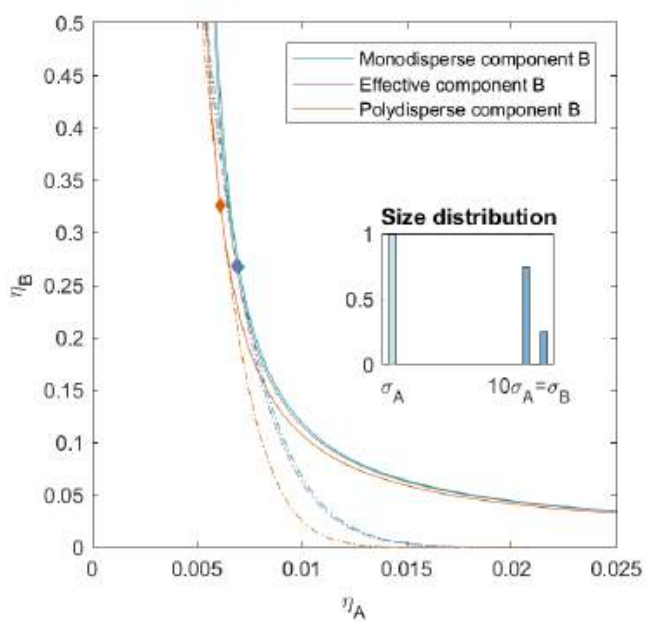

(b) Small amount of larger spheres

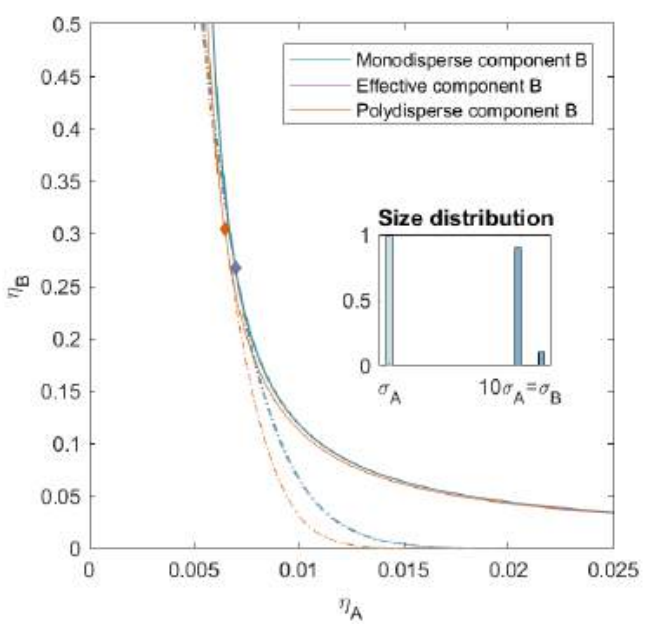

(d) Small amount of larger spheres

Figure 2.5: Phase diagram for binary (component $A$ and $B$ ) additive hard sphere mixture with size ratio $q=\sigma_{A} / \sigma_{B}=1 / 10$, component $A$ is monodisperse, component $B$ is polydisperse (see size distribution), with a number average size 10 times the size of component $A$, plotted as a function of the partial packing fractions, $\eta_{A}$ and $\eta_{B}$. The spinodal (solid line) and binodal (dashed line) meet each other at the critical point (diamond). 


\subsubsection{Polydispersity with 9 sub-components}

Now that we have a bit of an understanding of how polydispersity influences the critical point and the phase boundary, we increase the number of sub-components of $B$. The mixtures in Figure 2.6 thus consist of ten components (one component $A$ and nine components $B$ in varying amounts and sizes, with different degrees of polydispersity, dependent on the considered distribution). We again calculated the phase diagram using both the simplified effective $2 \times 2$ virial coefficient matrix (the effective mixture) and the full $10 \times 10$ virial coefficient matrix (the polydisperse mixture). Table 2.1 gives the critical points for the polydisperse mixtures and Table 2.2 allows for an easy comparison of the different distributions.

From the figure and table we can conclude that, the standard deviation of the polydisperse component plays a big role in moving the critical point and phase boundary. The standard deviation for $B$ in Figure 2.6b is twice the standard deviation for $B$ in Figure 2.6a. It is also clear that the type of distribution plays a significant role in the concentration of the critical point and position of the phase boundary. The sizes in $B$ for Figures $\mathbf{2 . 6 b}$ and $\mathbf{2 . 6 e}$ are the same, however, each size is present with a different frequency. The distribution in Figure 2.6b is Gaussian and the sizes in Figure 2.6e are bimodal, which means that the particles with sizes just larger and just smaller than the mean are present in a larger number. This causes to shift the critical point to slightly lower packing fraction of $B$ in Figure 2.6e compared to Figure 2.6b. The distribution of $B$ in mixture Figure $\mathbf{2 . 3}$ is comparable to the mixture in Figure 2.6a, just with fewer sub-components. The position of the critical point is for both mixtures very comparable. In the same way is the distribution of $B$ in Figure 2.4 comparable to the mixture in Figure 2.6b and Figure 2.6e. However, the increased number of sub-components results in a slightly higher and lower concentration of the $B$ component at the critical point respectively.

The distributions of $B$ in mixtures Figures 2.6c and 2.6d are skewed. In this regard they are comparable to the mixtures in Figure 2.5. For these mixtures we see that the right skewed distribution also causes the critical point to move to higher concentrations of $B$ compared to the mixture with the left skewed distribution.

\subsubsection{Fractionation of polydisperse component}

Upon phase separation, particles will move to a preferential phase in order to minimize the Helmholtz free energy. One phase is enriched in component $A$, whilst the other is enriched in component $B$. Even though each phase is enriched in one component, the other component is still present in lower concentrations. We investigated the phase separation of a specific parent mixture $\left(\eta_{A_{\text {parent }}}=0.010, \eta_{B_{\text {parent }}}=0.200\right)$ for the different distributions 


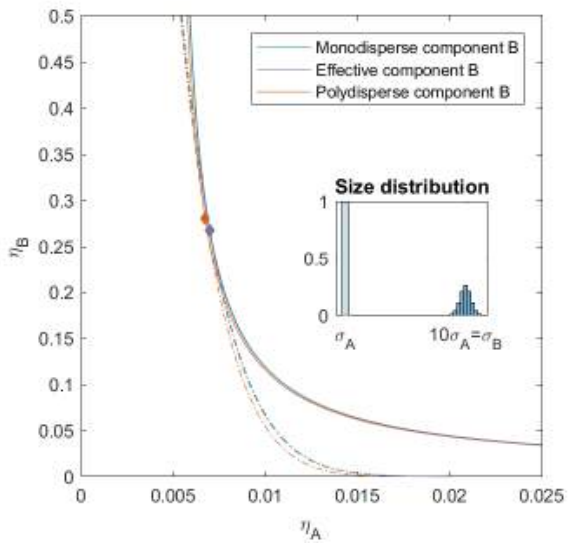

(a) Narrow Gaussian distribution

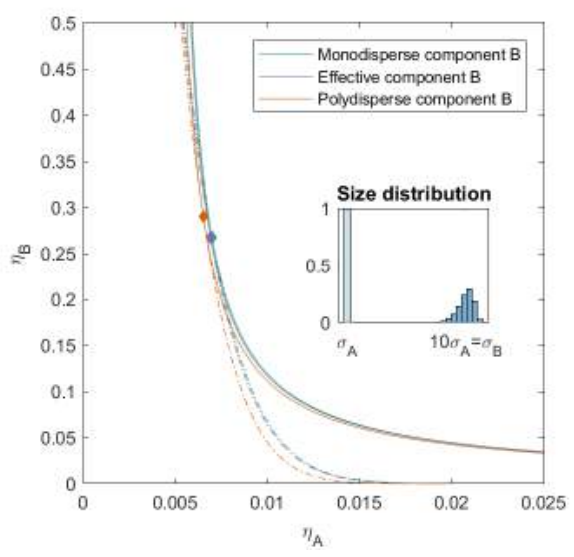

(c) Left skewed distribution

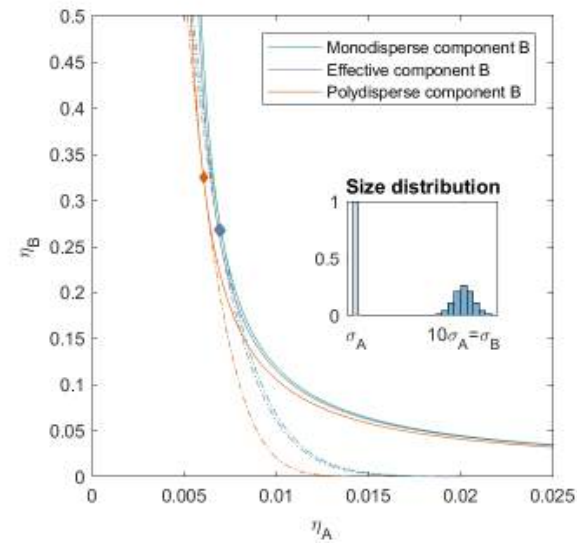

(b) Broad Gaussian distribution

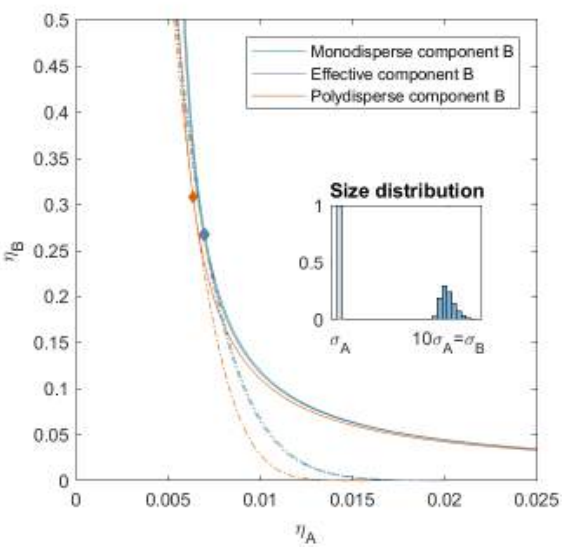

(d) Right skewed distribution

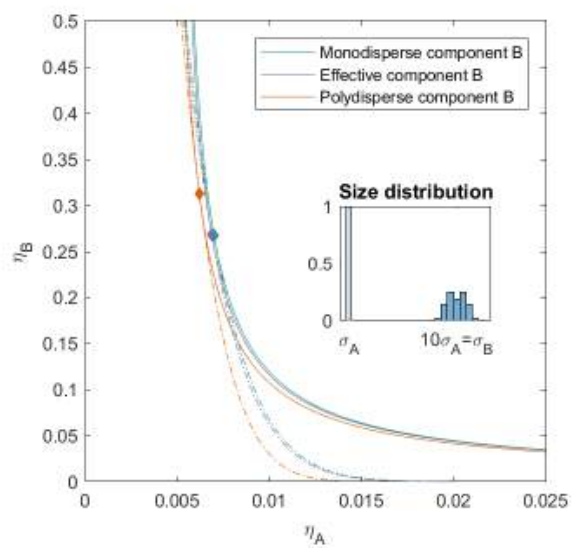

(e) Bimodal distribution

Figure 2.6: Phase diagram for binary (component $A$ and $B$ ) additive hard sphere mixture with size ratio $q=\sigma_{A} / \sigma_{B}=1 / 10$, component $A$ is monodisperse, component $B$ is polydisperse (see size distributions for the different distribution), with a number average size 10 times the size of component $A$, plotted as a function of the partial packing fractions, $\eta_{A}$ and $\eta_{B}$. The spinodal (solid line) and binodal (dashed line) meet each other at the critical point (diamond). 


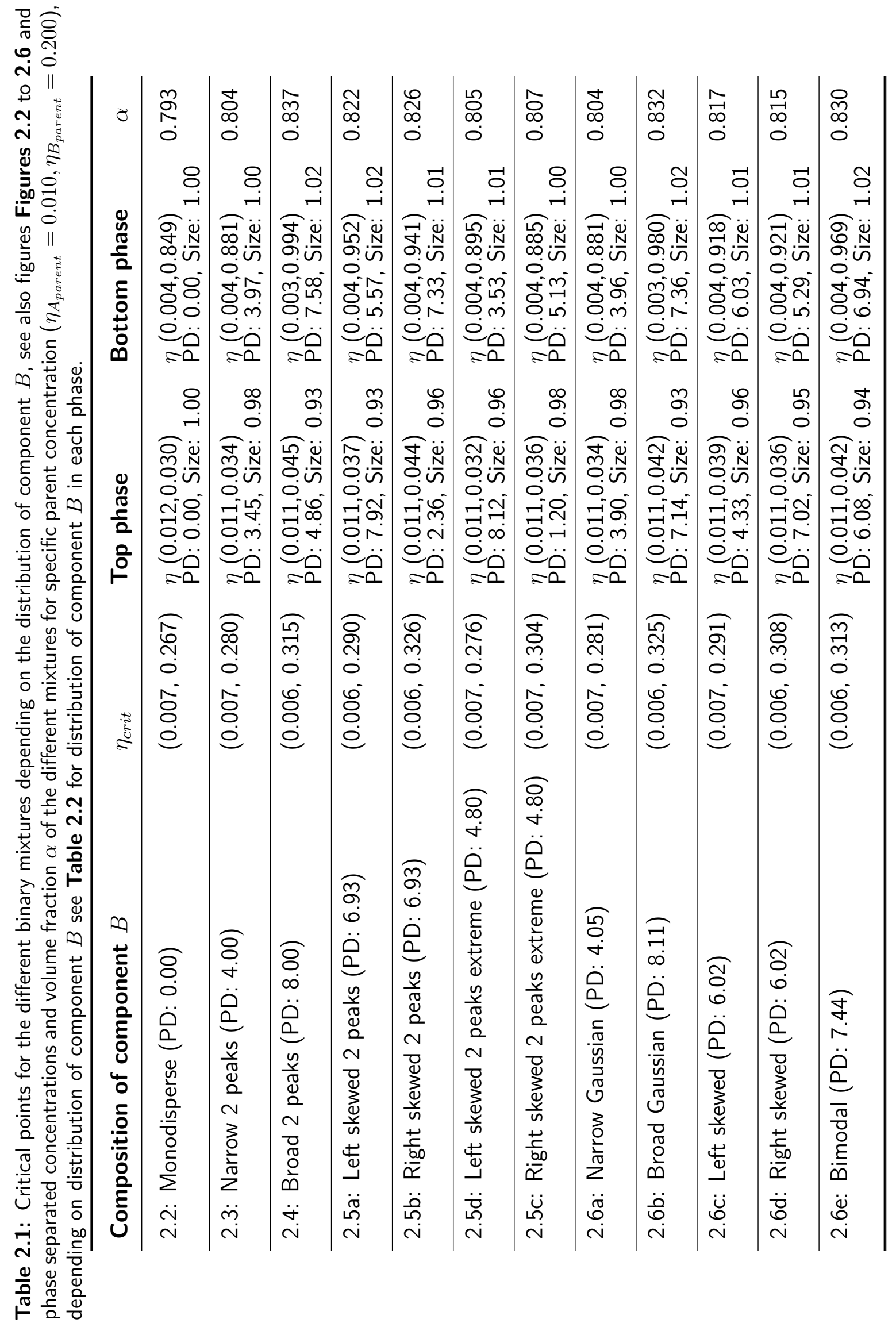


Table 2.2: Phase separation of different mixtures and fractionation of component $B$ for specific parent distribution $\left(\eta_{A_{\text {parent }}}=0.010, \eta_{B_{\text {parent }}}=0.200\right)$, depending on distribution of component $B$, see also figures Figures 2.2 to 2.6 .

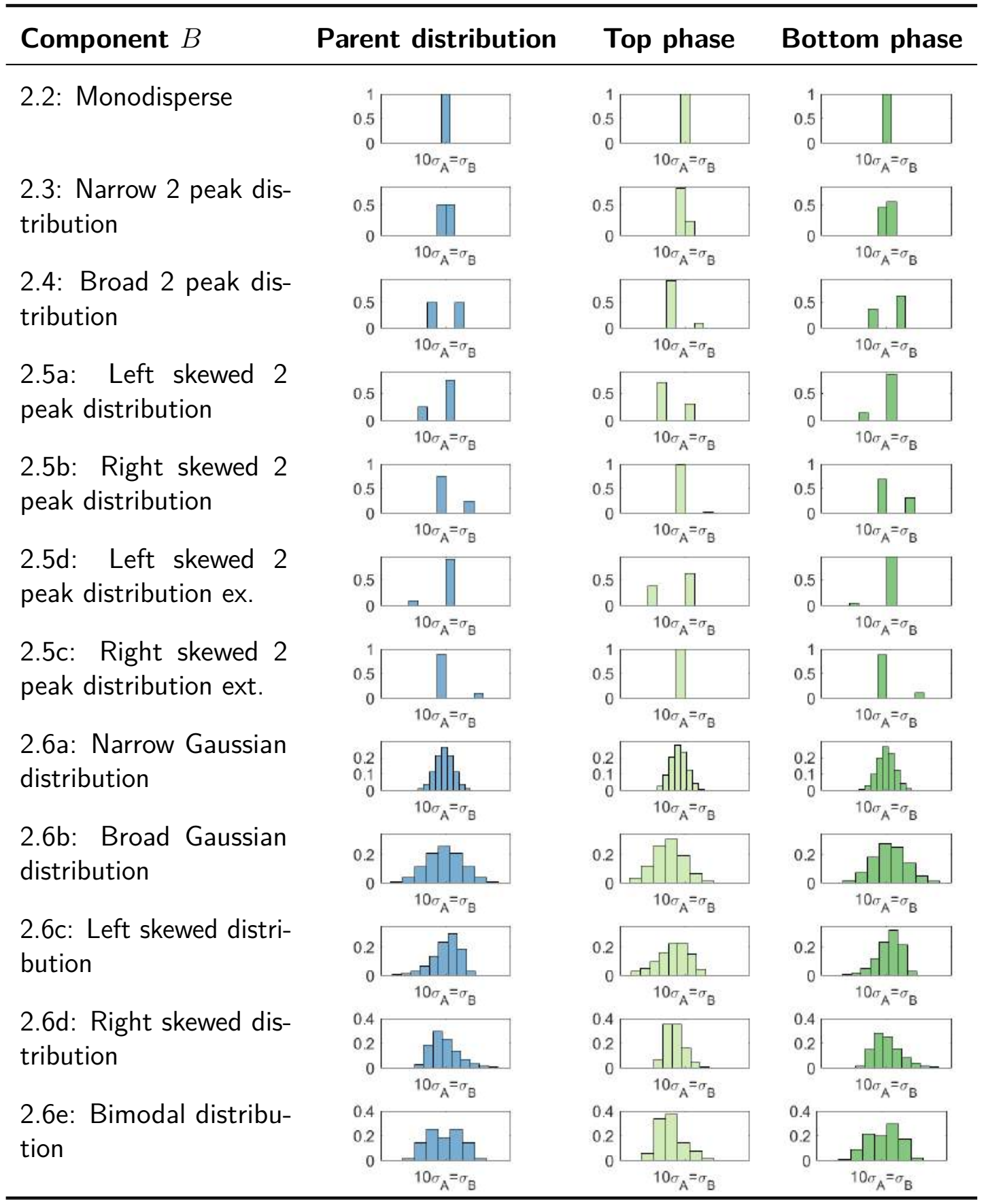


of component $B$, in terms of volume fraction of both components in each phase, degree of polydispersity of component $B$, average size of component $B$ in child phase compared to the average size of component $B$ in the parent phase and the volumefraction of the phases $(\alpha)$, see Table 2.2.

Polydispersity in the sizes of component $B$ in the parent phase causes significant fractionation of component $B$ in the child phases. The phase enriched in component $A$, the smaller component, contains also relatively more of the smaller components of $B$ than the phase enriched in component $B$. The size ratio of the average size of component $B$ compared to the average size of component $B$ in the parent phase is smaller than 1 . The polydispersity of component $B$ influences also both the composition in each phase as well as the volume fraction of the phases. The volume fraction of the top phase, the phase enriched in component $A$ (phase with lowest volume fraction in components) increases with polydipsersity. The bottom phase has a higher volume fraction of component $B$ with higher polydispersity. In general, but most pronounced for the mixture in Figure 2.5a, we observe that the smaller sub-components favor the top phase (the phase enriched in the small particles $A$ ), while the larger sub-components favor the bottom phase (the phase enriched in the larger particles $B$ ). We like to note that these observations as obtained from the virial approach are in line with previous theoretical work using the UNIQUAC model by Kang and Sandler (Kang and Sandler, 1988) and the Florry Huggins theory (van Heukelum et al., 2003) and also experimental work (Edelman et al., 2001; van der Kooij et al., 2000; Loret et al., 2005). Evans et al. (1998) also note that the skewness of the polydisperse parent distribution plays an important role in the fractionation. Also Paricaud (2008) found that the largest polydipserse particle favored the phase poor in the smallest particle. This follows from the relatively increased size incompatibility between the smallest particle and the largest particle in the system.

Depending on the type of distribution, phase separation causes the degree of polydispersity to decrease. This is in both phases for all mixtures with a symmetric distribution of $B$ in the parent phase. For these mixtures, the degree of polydispersity is at its lowest for the phase enriched in $A$.

\subsection{Conclusion}

We find that the largest species in the polydisperse component causes the largest shift in the position of the phase boundary, critical point and spinodal compared to the binary monodisperse binary mixtures. Upon phase separation, the polydisperse component fractionates. The smaller species of the polydisperse component favor the phase enriched in 
the small component, while the larger species remain in the phase enriched in the larger component. The top phase, the phase enriched in the small component, has a larger volume and this volume increases with polydispersity. The virial approach we used yields results in line with previous theoretical and experimental work on polydisperse mixtures, and at the same time allows for direct experimental testing using virial coefficients obtained from membrane osmometry.

\subsection{Acknowledgments}

We would like to thank Paul Venema and Arjen Bot for their interest in the work and the helpful discussions. 


\subsection{Bibliography}

M. Abramowitz and I. A. Stegun. Handbook of Mathematical Functions: With Formulas, Graphs, and Mathematical Tables. U.S. Department of Commerce, National Bureau of Standards, 1972.

B. L. Beegle, M. Modell, and R. C. Reid. Thermodynamic stability criterion for pure substances and mixtures. AIChE Journal, 20(6):1200-1206, 1974. ISSN 0001-1541. doi: 10.1002/aic.690200621.

L. Bellier-Castella, H. Xu, and M. Baus. Phase diagrams of polydisperse van der Waals fluids. Journal of Chemical Physics, 113(18):8337-8347, 2000. ISSN 00219606. doi: $10.1063 / 1.1316007$.

T. Biben and J.-P. Hansen. Spinodal instability of suspensions of large spheres in a fluid of small spheres. Journal of Physics: Condensed Matter, 3(42):F65-F72, 1991. ISSN 0953-8984. doi: 10.1088/0953-8984/3/42/006.

T. Biben and J.-P. Hansen. Osmotic depletion, non-additivity and phase separation. Physica A: Statistical Mechanics and its Applications, 235(1-2):142-148, 1997. ISSN 03784371. doi: 10.1016/S0378-4371(96)00335-4.

X. L. Chu, A. D. Nikolov, and D. T. Wasan. Effects of Particle Size and Polydispersity on the Depletion and Structural Forces in Colloidal Dispersions. Langmuir, 12(21):5004-5010, 1996. ISSN 0743-7463. doi: 10.1021/la960359u.

R. L. Cotterman and J. M. Prausnitz. Flash calculations for continuous or semicontinuous mixtures by use of an equation of state. Industrial \& Engineering Chemistry Process Design and Development, 24(2):434-443, 1985. ISSN 0196-4305. doi: 10.1021/i200029a038.

R. L. Cotterman, R. Bender, and J. M. Prausnitz. Phase Equilibria for Mixtures Containing Very Many Components. Development and Application of Continuous Thermodynamics for Chemical Process Design. Industrial and Engineering Chemistry Process Design and Development, 24(1):194-203, 1985. ISSN 01964305. doi: 10.1021/i200028a033.

M. Dijkstra, R. van Roij, and R. Evans. Phase diagram of highly asymmetric binary hardsphere mixtures. Physical Review E - Statistical Physics, Plasmas, Fluids, and Related Interdisciplinary Topics, 59(5):5744-5771, 1999. ISSN 1063651X. doi: 10.1103/PhysRevE.59.5744. 
M. W. Edelman, E. van der Linden, E. de Hoog, and R. H. Tromp. Compatibility of Gelatin and Dextran in Aqueous Solution. Biomacromolecules, 2(4):1148-1154, 2001. ISSN 1525-7797. doi: 10.1021/bm015545f.

C. Ersch, E. van der Linden, A. Martin, and P. Venema. Interactions in protein mixtures. Part II: A virial approach to predict phase behavior. Food Hydrocolloids, 52:991-1002, 2016. doi: 10.1016/j.foodhyd.2015.07.021.

R. M. Evans, D. J. Fairhurst, and W. C. Poon. Universal law of fractionation for slightly polydisperse systems. Physical Review Letters, 81(6):1326-1329, 1998. ISSN 10797114. doi: 10.1103/PhysRevLett.81.1326.

M. Fasolo and P. Sollich. Effects of polymer polydispersity on the phase behaviour of colloid-polymer mixtures. Journal of Physics: Condensed Matter, 17(6):797-812, 2005. ISSN 0953-8984. doi: 10.1088/0953-8984/17/6/002.

D. Goulding and J. P. Hansen. Effects of size polydispersity on depletion interactions. Molecular Physics, 99(10):865-874, 2001. ISSN 00268976. doi: 10.1080/00268970010018657.

J.-P. Hansen and I. McDonald. Theory of Simple Liquids, volume 21. Elsevier, 2013. ISBN 9780123870322. doi: 10.1016/C2010-0-66723-X.

R. A. Heidemann. The criteria for thermodynamic stability. AIChE Journal, 21(4):824-826, 1975. ISSN 0001-1541. doi: 10.1002/aic.690210433.

R. A. Heidemann. The Classical Theory of Critical Points. In Supercritical Fluids, pages 39-64. Springer Netherlands, 1994. doi: 10.1007/978-94-015-8295-7_2.

R. A. Heidemann and A. M. Khalil. The calculation of critical points. A/ChE Journal, 26 (5):769-779, 1980. ISSN 15475905. doi: 10.1002/aic.690260510.

T. L. Hill. An Introduction to Statistical Thermodynamics. Dover Publications, New York, 1986. ISBN 9780486652429.

P. Hopkins and M. Schmidt. Binary non-additive hard sphere mixtures: Fluid demixing, asymptotic decay of correlations and free fluid interfaces. Journal of Physics Condensed Matter, 22(32), 2010. ISSN 09538984. doi: 10.1088/0953-8984/22/32/325108.

C. H. Kang and S. I. Sandler. Effects of polydispersivity on the phase behavior of the aqueous two-phase polymer systems. Macromolecules, 21(10):3088-3095, 1988. ISSN 0024-9297. 
C. Loret, S. Schumm, P. D. Pudney, W. J. Frith, and P. J. Fryer. Phase separation and molecular weight fractionation behaviour of maltodextrin/agarose mixtures. Food Hydrocolloids, 19(3):557-565, may 2005. ISSN 0268005X. doi: 10.1016/j.foodhyd.2004.10.030.

W. G. McMillan and J. E. Mayer. The Statistical Thermodynamics of Multicomponent Systems. The Journal of Chemical Physics, 13(7):276-305, 1945. ISSN 0021-9606. doi: $10.1063 / 1.1724036$.

P. Paricaud. Phase equilibria in polydisperse nonadditive hard-sphere systems. Physical Review E, 78(2):021202, 2008. ISSN 1539-3755. doi: 10.1103/PhysRevE.78.021202.

M. Piech and J. Y. Walz. Effect of Polydispersity and Charge Heterogeneity on the Depletion Interaction in Colloidal Systems. Journal of Colloid and Interface Science, 225(1):134-146, 2000. ISSN 00219797. doi: 10.1006/jcis.2000.6755.

R. C. Reid and B. L. Beegle. Critical point criteria in legendre transform notation. A/ChE Journal, 23(5):726-732, 1977. ISSN 15475905. doi: 10.1002/aic.690230515.

A. Santos, M. López De Haro, and S. B. Yuste. Virial coefficients, thermodynamic properties, and fluid-fluid transition of nonadditive hard-sphere mixtures. Journal of Chemical Physics, 132(20), 2010. ISSN 00219606. doi: 10.1063/1.3429600.

R. P. Sear and D. Frenkel. Phase behavior of colloid plus polydisperse polymer mixtures. Physical Review E - Statistical Physics, Plasmas, Fluids, and Related Interdisciplinary Topics, 55(2):1677-1681, 1997. ISSN 1063651X. doi: 10.1103/PhysRevE.55.1677.

P. Sollich. Predicting phase equilibria in polydisperse systems. Journal of Physics Condensed Matter, 14(3):R79-R117, 2002. ISSN 09538984. doi: 10.1088/0953-8984/14/3/201.

M. A. Solokhin, A. V. Solokhin, and V. S. Timofeev. Phase-equilibrium stability criterion in terms of the eigenvalues of the Hessian matrix of the Gibbs potential. Theoretical Foundations of Chemical Engineering, 36(5):444-446, 2002. ISSN 00405795. doi: 10.1023/A:1020617610644.

M. R. Stapleton, D. J. Tildesley, and N. Quirke. Phase equilibria in polydisperse fluids. The Journal of Chemical Physics, 92(7):4456-4467, 1990. ISSN 0021-9606. doi: $10.1063 / 1.457756$.

R. Tuinier and A. V. Petukhov. Polymer Polydispersity Effect on Depletion Interaction between Colloidal Particles. Macromolecular Theory and Simulations, 11(9):975-984, 2002. ISSN 10221344. doi: 10.1002/1521-3919(200211)11:9<975::AID-MATS975>3.0.CO;2-Y. 
S. Vafaei, B. Tomberli, and C. G. Gray. McMillan-Mayer theory of solutions revisited: Simplifications and extensions. The Journal of Chemical Physics, 141(15):154501, 2014. ISSN 0021-9606. doi: 10.1063/1.4897980.

F. M. van der Kooij, M. Vogel, and H. N. W. Lekkerkerker. Phase behavior of a mixture of platelike colloids and nonadsorbing polymer. Physical Review E, 62(4):5397-5402, 2000. ISSN 1063-651X. doi: 10.1103/PhysRevE.62.5397.

A. van Heukelum, G. T. Barkema, M. W. Edelman, E. van der Linden, E. H. A. de Hoog, and R. H. Tromp. Fractionation in a Phase-Separated Polydisperse Polymer Mixture. Macromolecules, 36(17):6662-6667, 2003. ISSN 0024-9297. doi: 10.1021/ma025736q.

J. Y. Walz. Effect of Polydispersity on the Depletion Interaction between Colloidal Particles. Journal of Colloid and Interface Science, 178(2):505-513, 1996. ISSN 00219797. doi: 10.1006/jcis.1996.0145.

P. B. Warren. Fluid-fluid phase separation in hard spheres with a bimodal size distribution. Europhysics Letters, 46(3):295-300, 1999. ISSN 02955075. doi: 10.1209/epl/i199900259-y. 


\section{Effect of polydispersity on the phase behavior of non-additive hard spheres in solution, part II}

This chapter is based on: Luka Sturtewagen and Erik van der Linden. Effect of polydispersity on the phase behavior of non-additive hard spheres in solution, part II. (submitted) 


\begin{abstract}
We study the theoretical phase behavior of an asymmetric binary mixture of hard spheres, of which the smaller component is monodisperse and the larger component is polydisperse. The interactions are modeled in terms of the second virial coefficient and can be additive hard sphere (HS) or non-additive hard sphere (NAHS) interactions. The polydisperse component is subdivided into two sub-components and has an average size ten or three times the size of the monodisperse component. We give the set of equations that defines the phase diagram for mixtures with more than two components in a solvent. We calculate the theoretical liquid-liquid phase separation boundary for two phase separation (the binodal) and three phase separation, the plait point, and the spinodal. We vary the distribution of the polydisperse component in skewness and polydispersity, next to that we vary the non-additivity between the subcomponents as well as between the main components. We compare the phase behavior of the polydisperse mixtures with binary monodisperse mixtures for the same average size and binary monodisperse mixtures for the same effective interaction. We find that when the compatibility between the polydisperse sub-components decreases, three-phase separation becomes possible. The shape and position of the phase boundary is dependent on the non-additivity between the subcomponents as well as their size distribution. We conclude that it is the phase enriched in the polydisperse component that demixes into an additional phase when the incompatibility between the sub-components increases.
\end{abstract}




\subsection{Introduction}

In the study of the phase behavior of binary mixtures, the components are usually assumed to be pure and monodisperse, however in nature most components are not that neatly monodisperse. Many components show size and charge variation or contain hard to remove particles that can influence their phase behavior in binary mixtures. In their experimental work, Sager (1998) reported that even small impurities can lead to drastic shifts in the position of the phase boundary. Next to that the compatibility between components can be depended on the temperature (Edelman et al., 2001), salt concentration or $\mathrm{pH}$ of the solution (Kontogiorgos et al., 2009).

Two different physical mechanisms drive the phase separation between hard spheres. The first one involves only excluded volume interactions. In this mechanism the minimal distance between the particles is determined by the sum of their respective radii (Biben and Hansen, 1997). This is the typical additive hard sphere interaction (HS). With this mechanism, phase separation is driven by a size asymmetry between the particle sizes (Biben and Hansen, 1991). This asymmetry leads to depletion of small spheres around the large spheres and as a result to an effective attraction (depletion interaction) between the larger spheres (Dijkstra et al., 1999). The other mechanism is when the distance between the particles of a different species can be larger or smaller than the sum of their respective radii. This is referred to as non-additive hard sphere (NAHS) interaction. Previous research has shown that already at small degrees of non-additivity it becomes possible for components with no size asymmetry to demix (Roth et al., 2001; Dijkstra, 1998). Either way, upon phase separation, the mixture will demix into two (or more) phases, each enriched in one of the components. In the previous article, we focused on the first type of interaction (Sturtewagen and van der Linden, 2019). We investigated the influence of size polydispersity on the phase behavior of an additive binary asymmetric mixture. In this work we will focus on the second type, binary (polydisperse) mixtures where the distance between the particles of different species can be larger or smaller than the sum of their respective radii.

Piech and Walz (2000) studied the effect of size polydispersity and charge heterogeneity on the depletion interaction in a colloidal system. They found that the size distribution in the larger particle had a different effect on the depletion attraction for charged and non-charged hard sphere systems. For the depletion attraction decreased between the larger particles at constant volume fraction due to the polydispersity. This effect was further enhanced by the presence of charge. Polydispersity significantly lowers the magnitude of the repulsive barrier.

The non-additivity is usually described by the non-additivity parameter $\Delta$ (with $\Delta \geq-1$ ). When $\Delta=0$ the mixture has additive hard sphere interaction and the closest approach of the particles is the sum of their radii. When $\Delta<0$ the two particles experience more 
attraction and can come closer to each other than the sum of their radii, while when $\Delta>0$ the two particles have more repulsion and their distance of closest approach is larger than the sum of their respective radii. It is clear that this can have enormous effects on their phase behavior. Particles with a negative $\Delta$ tend to be more compatible with each other, while particles with a positive $\Delta$ are less compatible and tend to demix at lower concentrations. Already at the relatively low $\Delta=0.1$, it becomes possible for components with the same size to demix (Sillren and Hansen, 2010).

Paricaud (2008) studied the phase behavior of polydisperse colloidal dispersions. Their mixture consisted of a monodisperse component and a polydisperse component. The interaction between the monodisperse and polydisperse components was assumed to be NAHS (with the same $\Delta$ for all polydisperse sphere), while the interaction between the polydisperse components amongst themselves was assumed to be additive HS. They find that the critical point of a polydisperse mixture is at lower solution pressure than for completely monodisperse mixtures. For mixtures with large variation in the size of the polydisperse mixtures they observe the possibility of a three phase system. The phase behavior of a colloid and a polydisperse polymer was studied by Sear and Frenkel (1997). They used the Asakura-Osawa model fo the interactions between the different components. They found that increasing the polydispersity increased also the extent of the fluid-fluid coexistence. They reason that the introduction of larger polymer coils is the driving force towards phase separation.

In this study we aim to get a better understanding of how non-additive interaction influences the phase behavior of binary mixtures with some polydispersity or impurities. We will study the position of the phase separation boundary, the spinodal, and the critical point. Next to that we aim to predict the fractionation of the polydisperse component between the different phases. We model the interactions between the different components using the second virial coefficient (Section 3.2.1). In Section 3.2.2 we describe the equations for the spinodal, in Section 3.2.3 we describe the equations for the critical point and finally in Section 3.2.4 we describe the equations defining the phase boundary. With the expressions in Section 3.2 we have enough to calculate the phase diagram for a variety of mixtures described in Section 3.3. First we introduce non-additivity between the main components in the binary mixtures (Section 3.3.1), subsequently we introduce non-additivity between the sub-components in the polydiseperse component (Section 3.3.2), and finally we combine both in Section 3.3.3. In Section 3.3.4 we look into the fractionation of some of the mixtures from Section 3.3.2 at a specific parent concentration. 


\subsection{Theory}

We show the equations used for the calculations of the phase diagram of the different studied systems: the set of equations defining the stability boundary, the critical point, and phase boundaries of a mixture. All sets of equations are solved in Matlab R2017b. For a more detailed derivation of the equations, we refer to Sturtewagen and van der Linden (2019).

\subsubsection{Osmotic virial coefficient}

The osmotic pressure, $\Pi$, of a solution at a temperature $T$, can be written as a virial expansion, similar to the virial expansion of the universal gas law for real gasses (Hill, 1986):

$$
\beta \Pi=\rho+B_{2}\left(T, \mu_{s}\right) \rho^{2}+B_{3}\left(T, \mu_{s}\right) \rho^{3}+\ldots
$$

with $\beta=\frac{1}{k T}, k$ the Boltzmann's constant, $\rho$ the number density of the component $\left(\frac{N_{\nu}}{V}\right), B_{2}$ the second virial coefficient, and $B_{3}$ the third virial coefficient. The second virial coefficient accounts for the increase in osmotic pressure due to particle pairwise interaction The third virial coefficient accounts for the interaction between three particles in a variety of configurations. The equation can be expanded for higher densities with $B_{n}$, the $n^{\text {th }}$ virial coefficient, which accounts for the interaction between $n$ different particles.

In this work we will limit the virial expansion to the second virial coefficient, which is given by Lekkerkerker and Tuinier (2011):

$$
B\left(T, \mu_{s}\right)=2 \pi \int_{0}^{\infty} r^{2}(1-\exp [-\beta W(r)]) d r
$$

in which $\mu_{s}$ is the chemical potential of the solution, $W(r)$ is the interaction potential between the particles, and $r$ is the distance.

For additive hard sphere (HS) interaction, the interaction potential for two particles (of the same species or different species) is given by:

$$
W(r)_{H S}= \begin{cases}0, & r>\sigma_{i j} \\ \infty, & r \leq \sigma_{i j}\end{cases}
$$

with $\sigma_{i j}=\left(\sigma_{i}+\sigma_{j}\right) / 2$, the distance between the centers of the two particles.

For non-additive hard spheres (NAHS), the distance of the closest approach of the centers of the two particles of different species can be closer or further than the distance between their centers (Roth et al., 2001). The closest distance then becomes: $\sigma_{i j}=\left(\left(\sigma_{i}+\sigma_{j}\right) / 2\right)(1+\Delta)$, in which $\Delta(\geq-1)$ accounts for the non-additivity of the interaction between the particles. When $\Delta>0$ the distance of closest approach of both spheres increases and when $\Delta<0$ 
the distance of closest approach decreases compared to that due to HS interaction only. For additive hard sphere interaction $\Delta=0$.

In a mixture with $n$ distinguishable components in a solution, there are two main types of two particle interactions that can occur: between particles of the same species and particles of different species.

For the second virial coefficient given by Eq. (3.2), using the interaction potential defined in Eq. (3.3), we find:

$$
\begin{aligned}
& B_{x x}=\frac{2 \pi}{3}\left(\sigma_{x}\right)^{3} \\
& B_{x y}=\frac{2 \pi}{3}\left(\left(\frac{\sigma_{x}+\sigma_{y}}{2}\right)(1+\Delta)\right)^{3}
\end{aligned}
$$

where $B_{x x}$ is the second virial coefficient for particles of the same species (assumed to be HS) and $B_{x y}$ is the second virial coefficient of particles of different species, which can be HS or NAHS.

The general equation for the osmotic pressure for a dilute mixture is given by (Sturtewagen and van der Linden, 2019):

$$
\begin{aligned}
\beta \Pi & =\rho+B_{11} \rho_{1}^{2}+2 B_{12} \rho_{1} \rho_{2}+2 B_{13} \rho_{1} \rho_{3} \ldots \\
& =\rho+\sum_{i}^{n} \sum_{j}^{n} B_{i j} \rho_{i} \rho_{j}+\ldots
\end{aligned}
$$

In this article we focus on binary mixtures in which one of the components consists of sub-components (Figure 3.1). By increasing the number of sub-components, the number of equations to solve for the phase diagram increases. Just as in the previous article (Sturtewagen and van der Linden, 2019) we also compare the results to the number averaged virial coefficients of the different components. The number averaged virial coefficient was chosen because it allows for comparison to experiments, e.g. the virial coefficient obtained from osmometric measurements (Ersch et al., 2016).

The number averaged second virial coefficient of a mixture can be written as:

$$
\begin{aligned}
B_{\text {mix }} & =B_{11} x_{1}^{2}+2 B_{12} x_{1} x_{2}+2 B_{13} x_{1} x_{3} \ldots \\
& =\sum_{i}^{m} \sum_{j}^{m} B_{i j} x_{i} x_{j}
\end{aligned}
$$

in which $B_{i i}$ is the second virial coefficient of the $i^{\text {th }}$ particle, $B_{i j}$ is the second cross virial coefficient of the $i^{\text {th }}$ particle and the $j^{\text {th }}$ particle, and $x_{i}$ is the fraction of the $i^{\text {th }}$ particle, $\sum x_{i}=1$. 


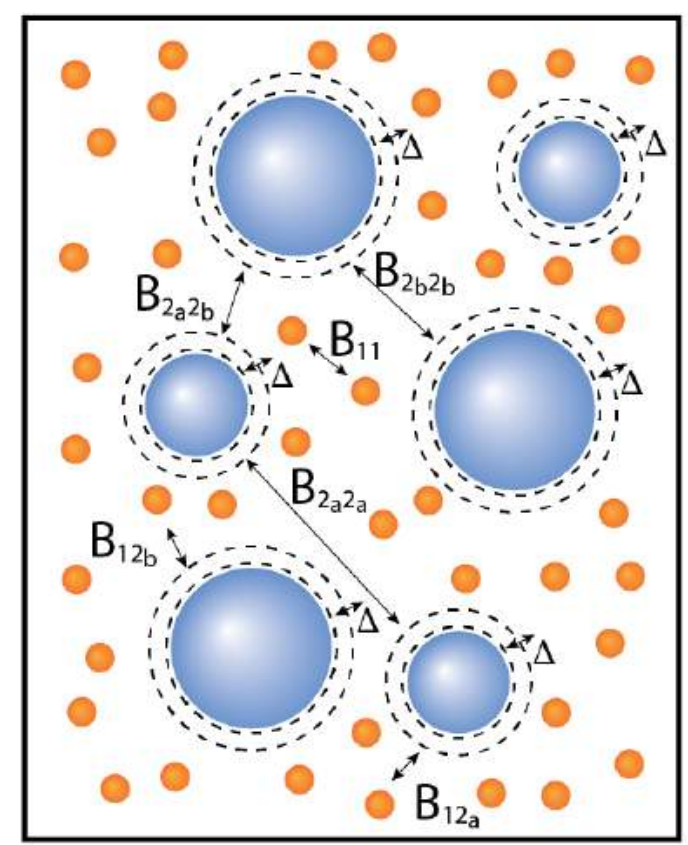

Figure 3.1: Graphical representation of a simple polydisperse mixture, in which the polydisperse component consists out of two sub-components ( $a$ and $b, n=3$ ), second virial coefficients are indicated. The distance of closest approach is influenced by $\Delta, \Delta>0$ increases this distance, $\Delta<0$ decreases this distance.

Using this definition, we can map the binary mixture consisting of for example a monodisperse component 1 and a component 2 subdivided into two subcomponents ( $a$ and b) by a $2 \times 2$ matrix of virial coefficients. We will refer to this $2 \times 2$ matrix as the effective virial coefficient matrix.

$$
\begin{aligned}
& B_{11_{\text {eff }}}=B_{11} \\
& B_{12_{\text {eff }}}=x_{a} B_{12_{a}}+x_{b} B_{12_{b}} \\
& B_{22_{\text {eff }}}=x_{a}^{2} B_{2_{a} 2_{a}}+2 x_{a} x_{b} B_{2_{a} 2_{b}}+x_{b}^{2} B_{2_{b} 2_{b}}
\end{aligned}
$$

The effective virial coefficient matrix for this mixture becomes then:

$$
B_{e f f}=\left[\begin{array}{ll}
B_{11_{\text {eff }}} & B_{12_{\text {eff }}} \\
B_{12_{\text {eff }}} & B_{22_{\text {eff }}}
\end{array}\right]
$$

\subsubsection{Stability of a mixture}

The stability of a mixture is dependent on the second derivative of the free energy. If the second derivative of the mixture becomes zero, the mixture is at the boundary of becoming 
unstable. Unstable mixtures have a negative second derivative (Heidemann, 1975; Beegle et al., 1974).

The differential of the free energy of a mixture is given by (Hill, 1986):

$$
d A=-S d T-p d V+\sum_{i}^{n} \mu_{i} d N_{i}
$$

in which $\mu_{i}$, the chemical potential (the first partial derivative of the free energy with respect to number of particles $\left(N_{i}\right)$ ) for component $i$ is given by:

$$
\mu_{i}=\mu_{i}^{0}+k T \ln \left(\rho_{i}\right)+2 k T\left(\sum_{j}^{n} B_{i j} \rho_{j}\right)
$$

For a mixture with $n$ distinguishable components, the second partial derivatives can be represented by a $n \times n$ matrix of the first partial derivatives of the chemical potential of each component.

This results in the following general stability matrix:

$$
\begin{aligned}
M_{1}= & {\left[\begin{array}{ccc}
\frac{\partial \mu_{1}}{\partial N_{1}} & \cdots & \frac{\partial \mu_{1}}{\partial N_{n}} \\
\vdots & \ddots & \vdots \\
\frac{\partial \mu_{n}}{\partial N_{1}} & \cdots & \frac{\partial \mu_{n}}{\partial N_{n}}
\end{array}\right] } \\
= & {\left[\begin{array}{ccc}
2 B_{11}+\frac{1}{\rho_{1}} & \cdots & 2 B_{1 n} \\
\vdots & \ddots & \vdots \\
2 B_{1 n} & \cdots & 2 B_{n n}+\frac{1}{\rho_{n}}
\end{array}\right] }
\end{aligned}
$$

The mixture is stable when all eigenvalues are positive (Solokhin et al., 2002), when on the other hand one of the eigenvalues is not positive, the mixture becomes unstable. The limit of stability is reached when the matrix has one zero eigenvalue and is otherwise positive definite, and is referred to as the spinodal (Heidemann and Khalil, 1980).

When there are only two components in the mixture $(n=2)$, the spinodal is defined by the condition det $M_{1}=0$. When the number of components is larger $(n>2)$, $\operatorname{det} M_{1}=0$ can have more than one solution (Solokhin et al., 2002). The spinodal can be found by checking whether $\operatorname{det} M_{1}$ changes sign for small changes in the concentrations of the components. 


\subsubsection{Critical points}

In a binary mixture, the critical point is a stable point which lies on the stability limit (spinodal) (Heidemann and Khalil, 1980) and where the phase boundary and spinodal coincide. In mixtures of more components these critical points become plait points. Critical points and plait points are in general concentrations at which two phases are in equilibrium and become indistinguishable (Heidemann, 1994).

There are two criteria that can be used to find critical points. The first one is $\operatorname{det}\left(M_{1}\right)=0$, which is the equation for the spinodal. The other criterion is based on the fact that at the critical point, the third derivative of the free energy should also be zero. For a multicomponent system, this criterion can be reformulated using Legendre transforms as $\operatorname{det}\left(M_{2}\right)=0$ (Beegle et al., 1974; Reid and Beegle, 1977), where:

$$
M_{2}=\left[\begin{array}{ccc}
\frac{\partial \mu_{1}}{\partial N_{1}} & \cdots & \frac{\partial \mu_{n}}{\partial N_{n}} \\
\vdots & \ddots & \vdots \\
\frac{\partial M_{1}}{\partial N_{1}} & \cdots & \frac{\partial M_{1}}{\partial N_{n}}
\end{array}\right]
$$

Matrix $M_{2}$ is matrix $M_{1}$ with one of the rows replaced by the partial derivatives of the determinant of matrix $M_{1}$. Note: it does not matter which row of the matrix is replaced.

\subsubsection{Phase boundary}

When a mixture becomes unstable and demixes into two or more phases, the chemical potential of each component and the osmotic pressure is the same in all phases (Hill, 1986).

$$
\left\{\begin{array}{c}
\beta \Pi^{I}=\beta \Pi^{I I}=\cdots \\
\beta \mu_{1}^{I}=\beta \mu_{1}^{I I}=\cdots \\
\vdots \\
\beta \mu_{n}^{I}=\beta \mu_{n}^{I I}=\cdots
\end{array}\right.
$$

where the phases are denoted by $I, I I, \ldots$.

For a mixture containing $n$ distinguishable components, that demixes into two phases, this results in $n+1$ equations and $2 \times n$ unknowns. If the mixture demixes into three phases, this results in $2 \times n+2$ equations and $3 \times n$ unknowns. To solve the set of equations without having to fix the concentration of one component and the ratio between the other components for at least one of the phases, we need extra equations. For the extra set of equations, we build on the fact that no particles are lost and no new particles are created during phase separation, and the fact that we assume that the total volume does not change. 
For a system that separates into three phases we then obtain:

$$
\rho=\sum_{i}^{n} \rho_{i}=\frac{\sum_{i}^{n} N_{i}}{V}=\frac{\sum_{i}^{n} N_{i}^{I}+\sum_{i}^{n} N_{i}^{I I}+\sum_{i}^{n} N_{i}^{I I I}}{V^{I}+V^{I I}+V^{I I I}}
$$

which can be rewritten as (Sturtewagen and van der Linden, 2019):

$$
\rho=\alpha^{I} \sum_{i}^{n} \rho_{i}^{I}+\alpha^{I I} \sum_{i}^{n} \rho_{i}^{I I}+\left(1-\alpha^{I}-\alpha^{I I}\right) \sum_{i}^{n} \rho_{i}^{I I I}
$$

with

$$
\alpha^{I}=\frac{V^{I}}{\sum_{i}^{f} V^{i}}
$$

in which $f$ denotes the number of phases.

This results in the following set of equations:

$$
\left\{\begin{aligned}
\beta \Pi^{I} & =\beta \Pi^{I I}=\cdots \\
\beta \mu_{1}^{I} & =\beta \mu_{1}^{I I}=\cdots \\
\vdots & \\
\beta \mu_{n}^{I} & =\beta \mu_{n}^{I I}=\cdots \\
\rho_{1} & =\alpha^{I} \rho_{1}^{I}+\cdots+\left(1-\sum_{i}^{f-1} \alpha^{i}\right) \rho_{1}^{f} \\
\vdots & \\
\rho_{n} & =\alpha^{I} \rho_{n}^{I}+\cdots+\left(1-\sum_{i}^{f-1} \alpha^{i}\right) \rho_{n}^{f}
\end{aligned}\right.
$$

With this set of equations, we have $2 \times n+1$ unknowns and $2 \times n+1$ equations for mixtures that separate into two phases. For mixtures that demix into three phases, we have $3 \times n+2$ unknowns and $3 \times n+2$ equations. Therefore, this set of equations allows for calculating the concentration of each component in each of the phases for any given parent concentration, given that the mixture will demix at this concentration.

\subsection{Results and discussion}

In this work we calculated the phase diagram for a variety of binary non-additive mixtures of a small hard sphere $A$ and a larger hard sphere $B$ with a size ratio $q=\sigma_{A} / \sigma_{B}$. Component 
$B$ is sub-divided into two sub-components and is characterized by a degree in polydispersity $(P D)$, defined by:

$$
P D=\frac{\sqrt{\sum\left(\sigma_{B_{i}}-\sigma_{B}\right)^{2} \times N_{B_{i}} / N_{B}}}{\sigma_{B}} \times 100
$$

We varied the non-additivity between particles of component $A$ and $B\left(\Delta_{A B}\right)$, and between the sub-components of $B\left(\Delta_{B_{a} B_{b}}\right)$. Next to that we varied the degree of polydispersity $(P D)$ of component $B$ and the distribution between the sub-components as well as the size ratio $(q)$ between component $A$ and $B$.

For all particles, the concentrations are expressed as a dimensionless parameter according to $\eta=\frac{\pi \rho \sigma^{3}}{6}$. We calculated the critical point, the phase separation boundary, and the spinodal of the various mixtures. Next to that, we also investigated the composition of the child phases, volume fraction of the phases $(\alpha)$, and the fractionation of the polydisperse component $B$ for a specific parent mixture $(\eta=(0.010,0.200))$, for mixtures with a size ratio $q=\sigma_{A} / \sigma_{B}=1 / 10$ and $\Delta_{A B}=0$, while varying the non-additive interaction between the sub-components of $B\left(\Delta_{B_{a} B_{b}}\right)$.

\subsubsection{Non-additive interaction between component $A$ and $B\left(\Delta_{A B}\right)$}

For the first set of mixtures (see Figure 3.2), we calculated the phase diagram for binary mixtures with non-additive interaction between monodisperse component $A$ and slightly polydisperse component $B$, with two sub-components and a $P D=4.00$. These two sub-components are additive hard spheres in two sizes (both present in the same amount), with the number average size of the mixture equal to 10 times the size of component $A$. The mixture therefore consist of three components of different size. We varied the non-additivity between component $A$ and $B$ ( $\Delta_{A B}$, the same for both sub-components) from - 0.1 to 0.5 with a step size of 0.1 . When $\Delta_{A B}=0$, the interaction between all components equals additive hard sphere interaction. We calculated the phase diagram using both the simplified $2 \times 2$ effective virial coefficient matrix described in the theory (we refer to this as the effective mixture $B$ ) and the full $3 \times 3$ virial coefficient matrix (to which we refer as the polydisperse mixture $B$ ). These mixtures are also compared to mixtures in which component $B$ is monodisperse with a size equal to the average particle size of component $B$ (we refer to this as the monodisperse mixture $B$ ).

With increasing $\Delta_{A B}$, the phase boundary, spinodal and critical point shifts towards lower concentrations, for the monodisperse mixture, effective mixture, and polydisperse mixture. This is in line with research on non-additive binary mixtures Hopkins and Schmidt (2010). The difference between the phase boundary, spinodal and critical point of the 


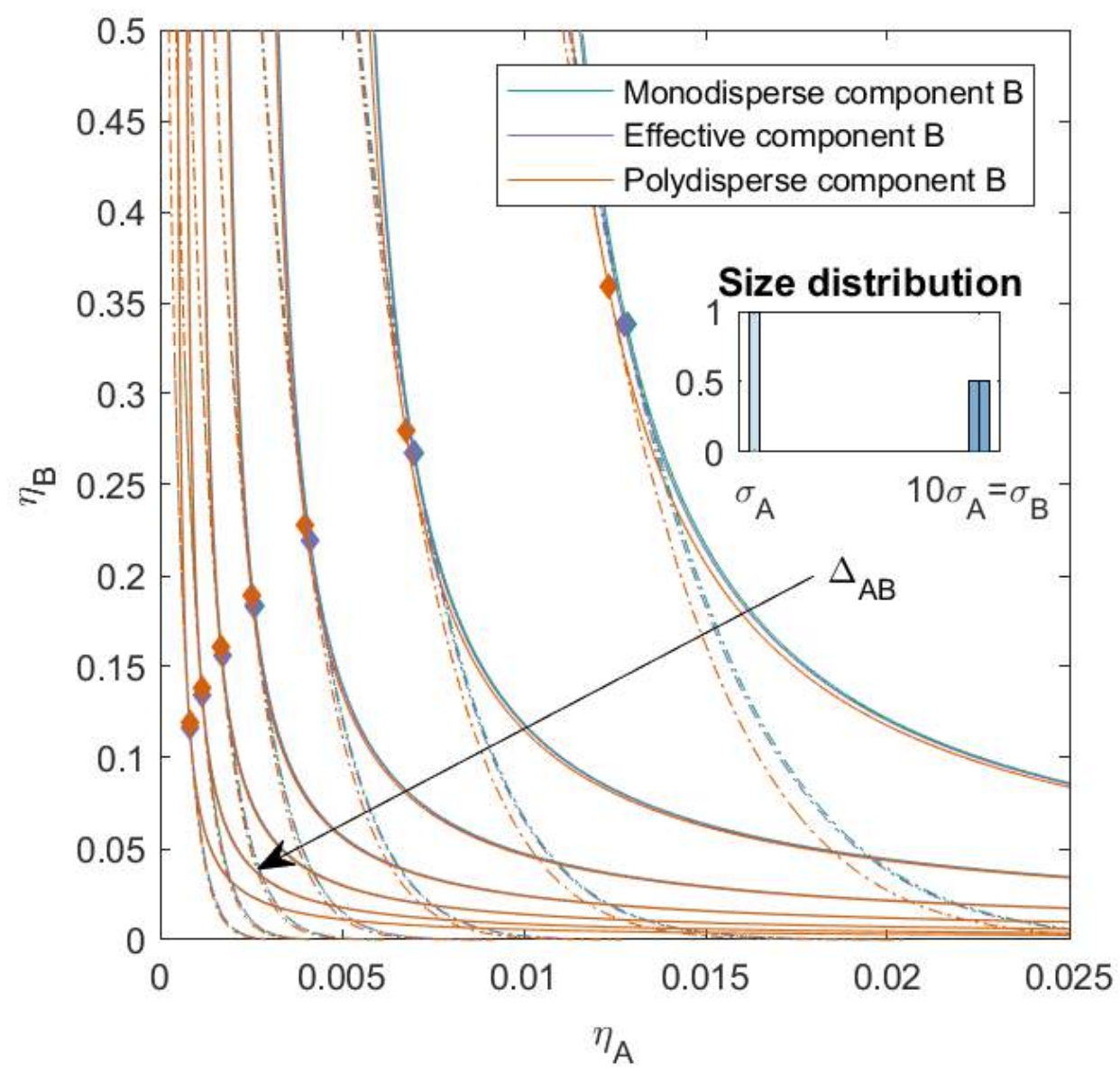

Figure 3.2: Phase diagram for binary (component $A$ and $B$ ) non-additive hard sphere mixture with size ratio $q=\sigma_{A} / \sigma_{B}=1 / 10$, component $A$ is monodisperse, component $B$ is polydisperse $(P D=4.00)$, plotted as a function of the partial packing fractions, $\eta_{A}$ and $\eta_{B}$. The interaction between components $A$ and $B$ is non-additive, the non-additivity parameter $\Delta_{A B}$ was varied from -0.1 to 0.5 with a step size of 0.1 (the arrow indicates increasing $\Delta_{A B}$ ). The interaction between the sub-components $B$ is additive. The spinodal (solid line) and binodal (dashed line) meet each other at the critical point (diamond).

monodisperse mixture and the effective mixture is negligible, for all $\Delta_{A B}$. We see however that the introduction of the polydispersity causes the critical point to shift to a higher volume fraction of component $B$ and that especially at lower volume fraction of component $B$ the phase separation boundary shifts towards slightly lower packing fractions. This effect is more pronounced when $\Delta_{A B}$ is small.

When the $P D$ of component $B$ is increased, or the distribution of the sub-components of $B$ is varied, we see the same pattern as in Figure 3.2 (see supplementary materials). However, we see that, just like discussed in Sturtewagen and van der Linden (2019), the critical point shifts towards higher concentrations of $B$ for the polydisperse mixtures depending on the size and concentration of the largest sub-component of $B$ and the difference between the effective and the monodisperse mixtures increases with the size of the largest 

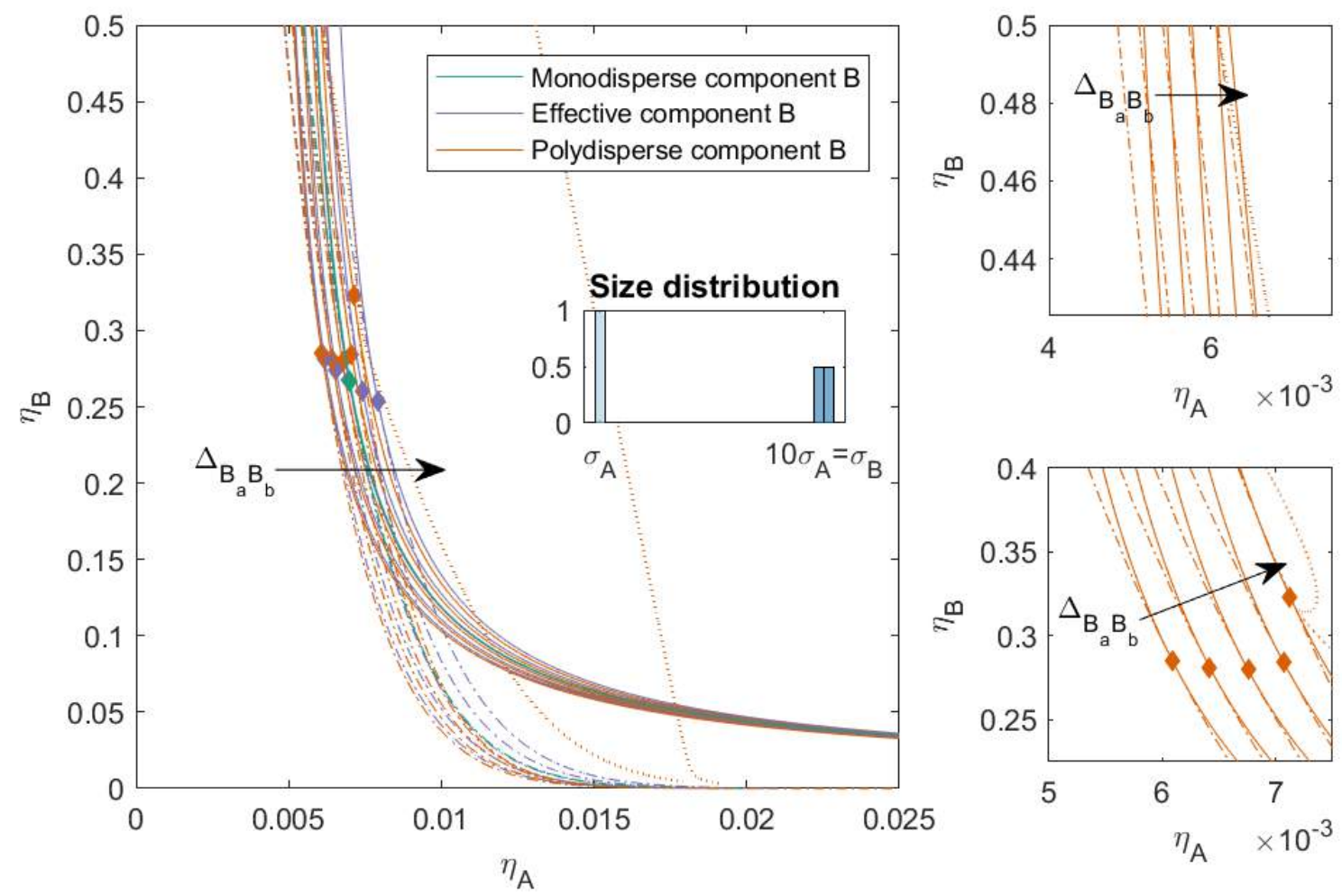

Figure 3.3: Phase diagram for binary (component $A$ and $B$ ) non-additive hard sphere mixture with size ratio $q=\sigma_{A} / \sigma_{B}=1 / 10$, component $A$ is monodisperse, component $B$ is polydisperse $(P D=4.00)$, plotted as a function of the partial packing fractions, $\eta_{A}$ and $\eta_{B}$. The interaction between components $A$ and $B$ is additive, the interaction between the sub-components $B$ is non-additive, the non-additivity parameter $\Delta_{B_{a} B_{b}}$ was varied from -0.1 to 0.1 with a step size of 0.05 (the arrow indicates increasing $\Delta_{B_{a} B_{b}}$ ). The spinodal (solid line) and binodal (dashed line) meet each other at the plait point (diamond), the three phase boundary is indicated with a dotted line and meets the spinodal at the plait point (diamond).

sub-component of $B$.

\subsubsection{Non-additive interaction within polydisperse component $B$ $\left(\Delta_{B_{a} B_{b}}\right)$}

In the next set of mixtures, we kept the interaction between the components $A$ and $B$ as hard-sphere additive, but we introduced some non-additivity in the interaction between the sub-components of $B$. We varied $\Delta_{B_{a} B_{b}}$ from -0.10 to 0.10 with a step size of 0.05 . When $\Delta_{B_{a} B_{b}}$ is small, the sub-components are more compatible with each other, when on the other hand $\Delta_{B_{a} B_{b}}$ increases and becomes positive, the compatibility between the sub-components decreases. When $\Delta_{B_{a} B_{b}}>0$ it becomes possible for components of similar size to phase separate (Hopkins and Schmidt, 2010).

In Figure 3.3 we plotted the phase diagram for the binary mixtures with $P D=4.00$, 

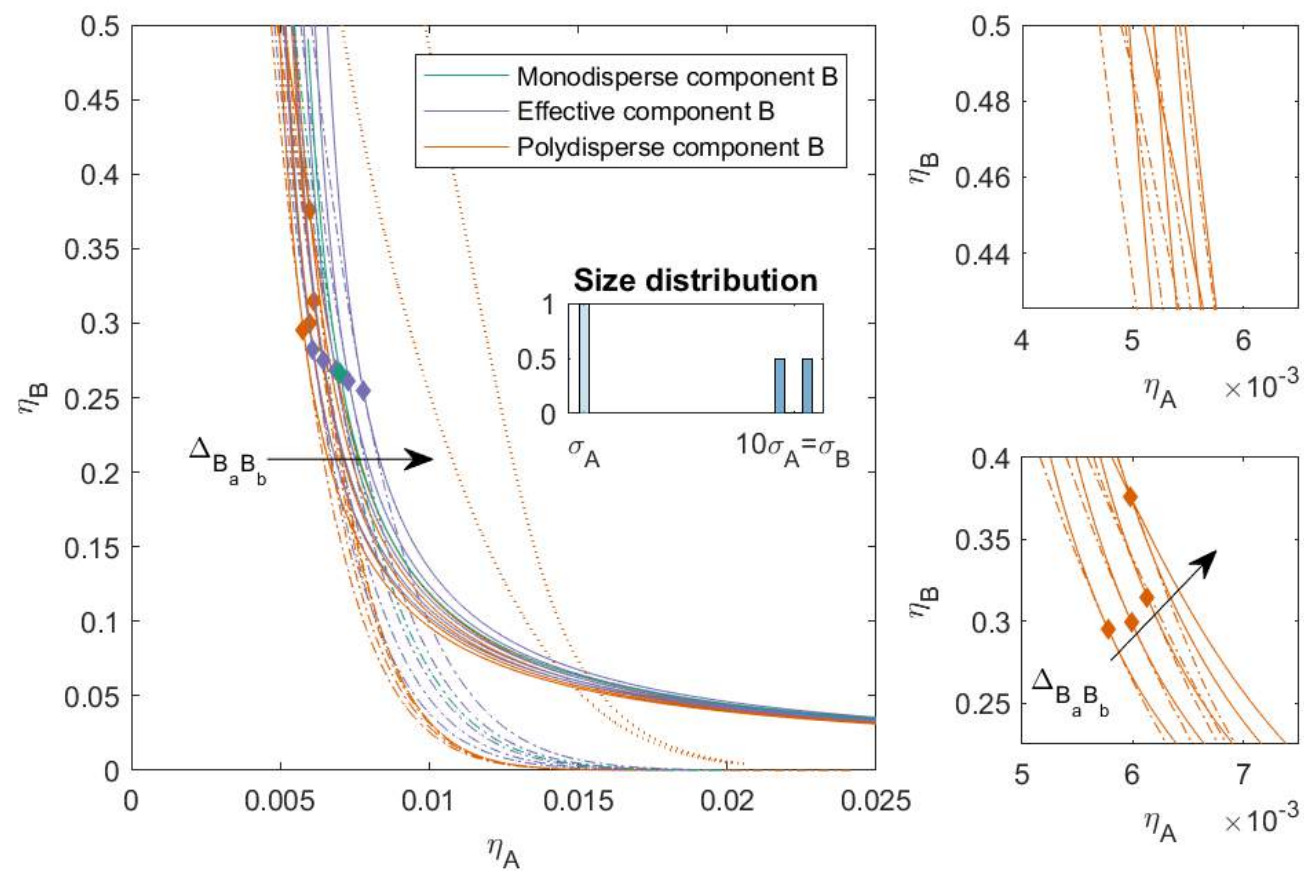

(a) $P D=8.00$
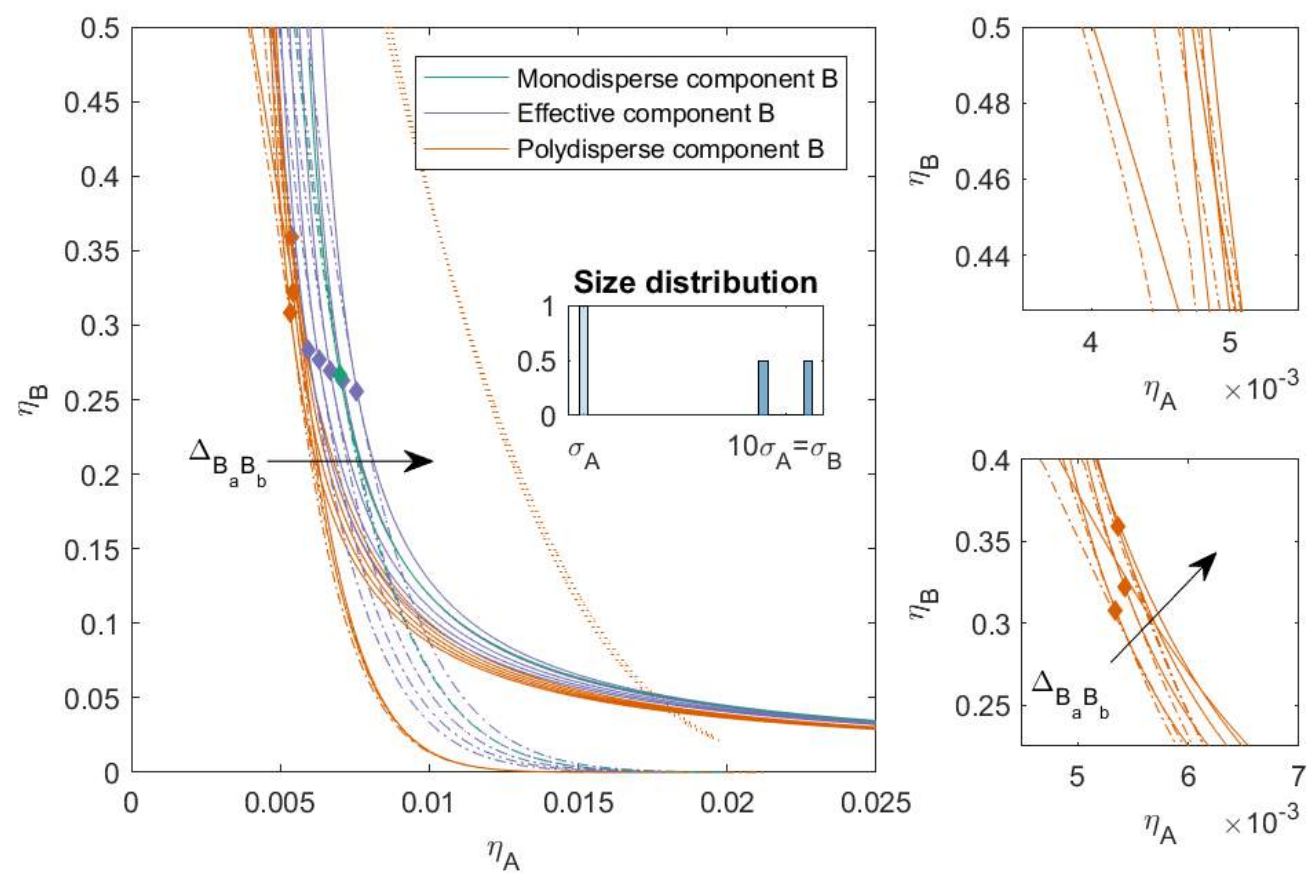

(b) $P D=12.00$

Figure 3.4: Phase diagram for binary (component $A$ and $B$ ) non-additive hard sphere mixture with size ratio $q=\sigma_{A} / \sigma_{B}=1 / 10$, component $A$ is monodisperse, component $B$ is polydisperse $(P D=8.00$ or $P D=12.00)$, plotted as a function of the partial packing fractions, $\eta_{A}$ and $\eta_{B}$. The interaction between components $A$ and $B$ is additive, the interaction between the sub-components $B$ is non-additive, the non-additivity parameter $\Delta_{B_{a} B_{b}}$ was varied from -0.1 to 0.1 with a step size of 0.05 (the arrow indicates increasing $\Delta_{B_{a} B_{b}}$ ). The spinodal (solid line) and binodal (dashed line) meet each other at the plait point (diamond), the three phase boundary is indicated with a dotted line. 
$\Delta_{A B}=0$ and we varied $\Delta_{B_{a} B_{b}}$. When $\Delta_{B_{a} B_{b}}>0$, the compatibility between the subcomponents decreases and phase separation into three phases becomes possible (depicted as the dotted line in the figure). Mixtures with a smaller $\Delta_{B_{a} B_{b}}$ demix into two phases at lower concentrations compared to the completely hard sphere mixture. Mixtures with a larger $\Delta_{B_{a} B_{b}}$ demix into two phases at higher packing fractions compared to the completely hard sphere mixture, and also have a three phase boundary. The three-phase boundary is at lower concentrations for larger $\Delta_{B_{a} B_{b}}$ and comes close to the two-phase boundary for the mixture with $\Delta_{B_{a} B_{b}}=0.10$. The critical point of the polydisperse mixtures changes depending on the non-additivity of the sub-components: The critical point is at its lowest concentrations of $A$ for negative $\Delta_{B_{a} B_{b}}$, its lowest concentration of $B$ is when the interaction between the sub-components of $B$ becomes more like HS, and the concentration of the critical point for $B$ increases with $\Delta_{B_{a} B_{b}}$.

In Figure 3.4 we increased the $P D$ for component $B$ to 8.00 and 12.00 respectively, we kept $\Delta_{A B}=0$ and we varied $\Delta_{B_{a} B_{b}}$ as before. With increased $P D$, the two-phase boundary of the polydisperse mixture shifts towards lower packing fractions for all mixtures. The effect of $\Delta_{B_{a} B_{b}}$ on the position of the two-phase boundary becomes smaller at lower concentration of $B$, however at higher concentrations of $B$ we see that the two-phase boundary for positive $\Delta_{B_{a} B_{b}}$ bends towards the y-axis and this effect is more pronounced for higher $P D$. The polydispersity of $B$ also has an effect on the position of the three-phase boundary. With increased $P D$, the position of the three-phase boundary becomes less dependent on $\Delta_{B_{a} B_{b}}$ and the difference in the position of the two-phase boundary and the three-phase boundary increases for the mixtures with $\Delta_{B_{a} B_{b}}=0.10$. For the mixtures with $P D=12.00$, the difference between the three-phase boundary for the mixtures that phase separate into three phases becomes negligible. We see similar trends in the critical points for the more polydisperse mixture as in Figure 3.3, however, with increased polydispersity and especially increased incompatibility between the sub-components $\left(\Delta_{B_{a} B_{b}}>0\right)$, the critical point shifts towards higher concentrations of $B$. For the mixtures with larger $\Delta_{B_{a} B_{b}}$, the critical point can shift to $\eta_{B_{\text {crit }}}>0.5$.

In Figures $\mathbf{3 . 5}$ and $\mathbf{3 . 6}$ we varied the ratio between the sub-components of $B$. The ratio between the sub-components of $B$ is $25 / 75$ with a $P D=6.93$ in Figure 3.5 (both left and right skewed) and 10/90 with a $P D=4.80$ in Figure 3.6 (both left and right skewed). These mixtures can be seen as a model for mixtures that contain some impurities, from a similiar material but at at different size when $\Delta_{B_{a} B_{b}}=0$ or a material that is less compatible with the main component (when $\Delta_{B_{a} B_{b}}>0$ ) or more compatible with the main component (when $\Delta_{B_{a} B_{b}}<0$ ). The $P D$ is the same for both the left skewed and the right skewed mixtures. For both types of mixtures, we see that the two-phase boundaries are closer to each other for the left-skewed mixtures (large amount of the largest sub-component) 

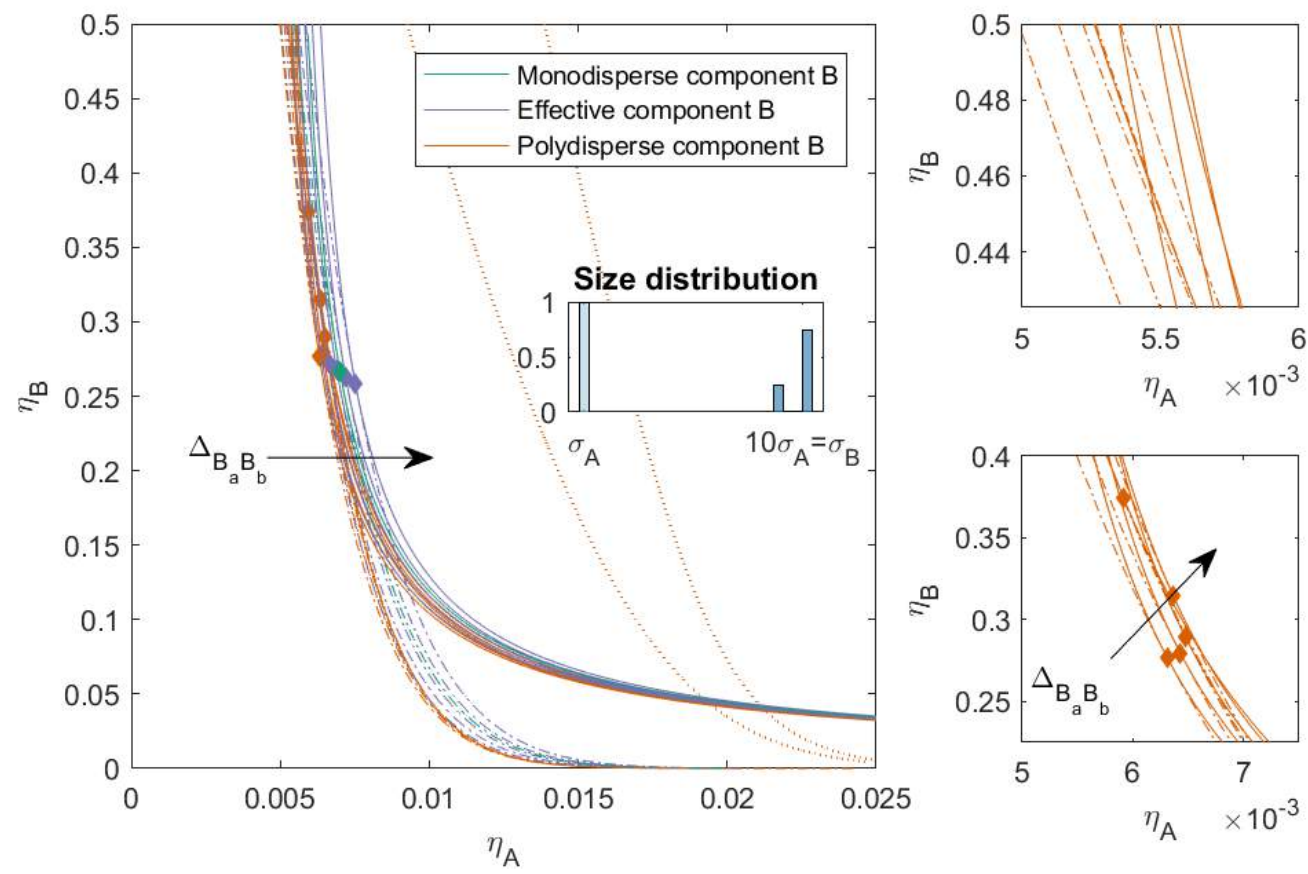

(a) Left skewed
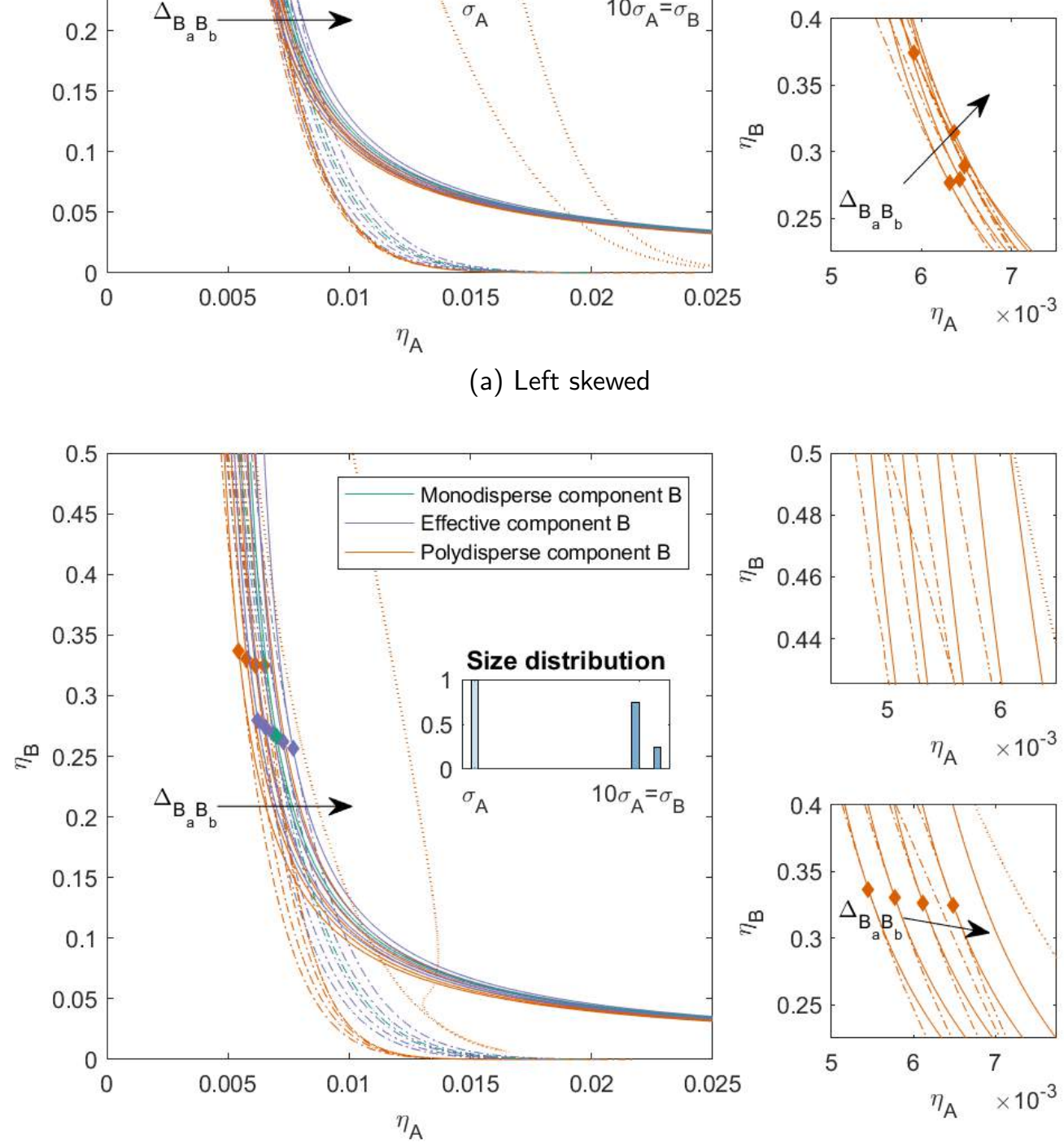

(b) Right skewed

Figure 3.5: Phase diagram for binary (component $A$ and $B$ ) non-additive hard sphere mixture with size ratio $q=\sigma_{A} / \sigma_{B}=1 / 10$, component $A$ is monodisperse, component $B$ is polydisperse $(P D=6.93)$, plotted as a function of the partial packing fractions, $\eta_{A}$ and $\eta_{B}$. The interaction between components $A$ and $B$ is additive, the interaction between the sub-components $B$ is non-additive, the non-additivity parameter $\Delta_{B_{a} B_{b}}$ was varied from -0.1 to 0.1 with a step size of 0.05 (the arrow indicates increasing $\Delta_{B_{a} B_{b}}$ ). The spinodal (solid line) and binodal (dashed line) meet each other at the plait point (diamond), the three phase boundary is indicated with a dotted line. 

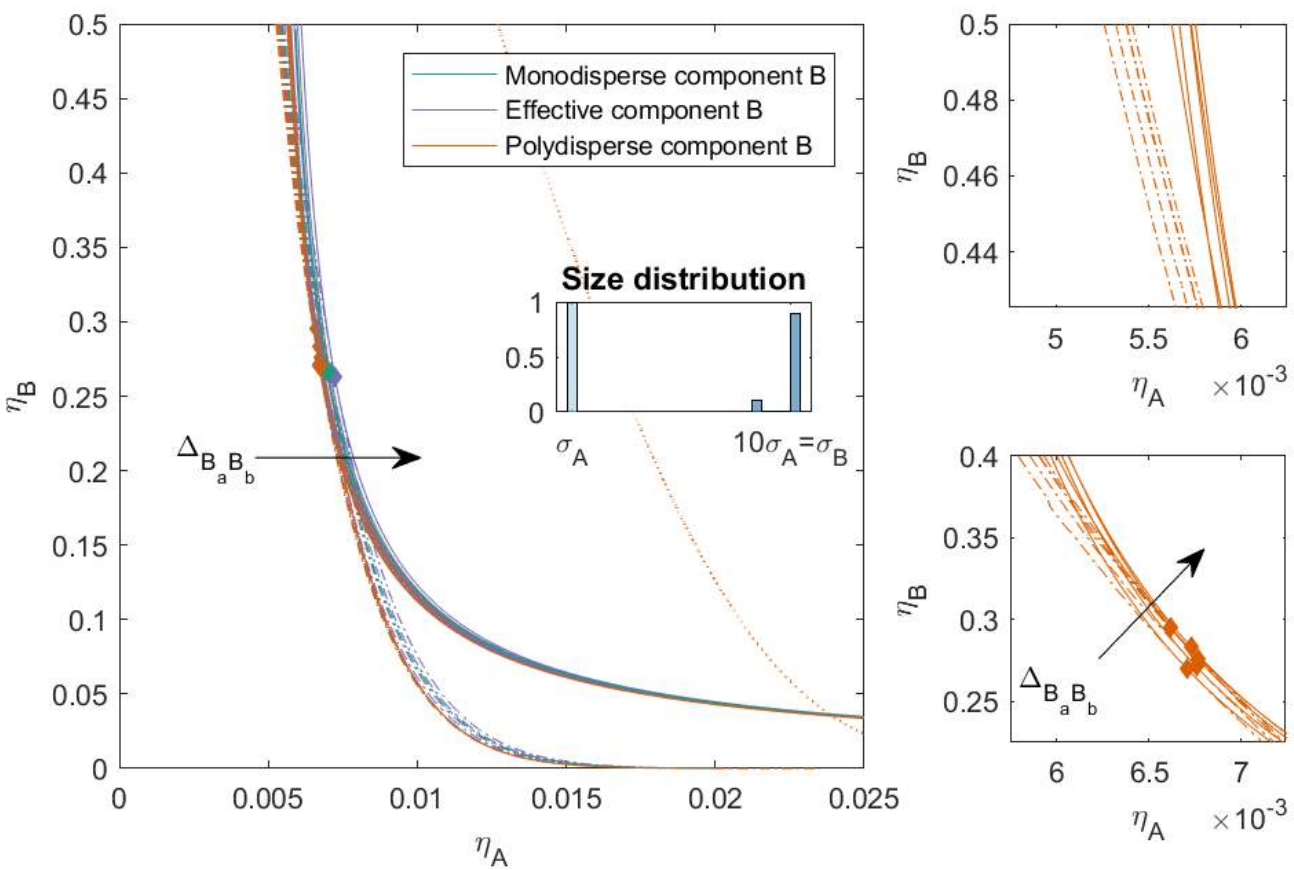

(a) Left skewed
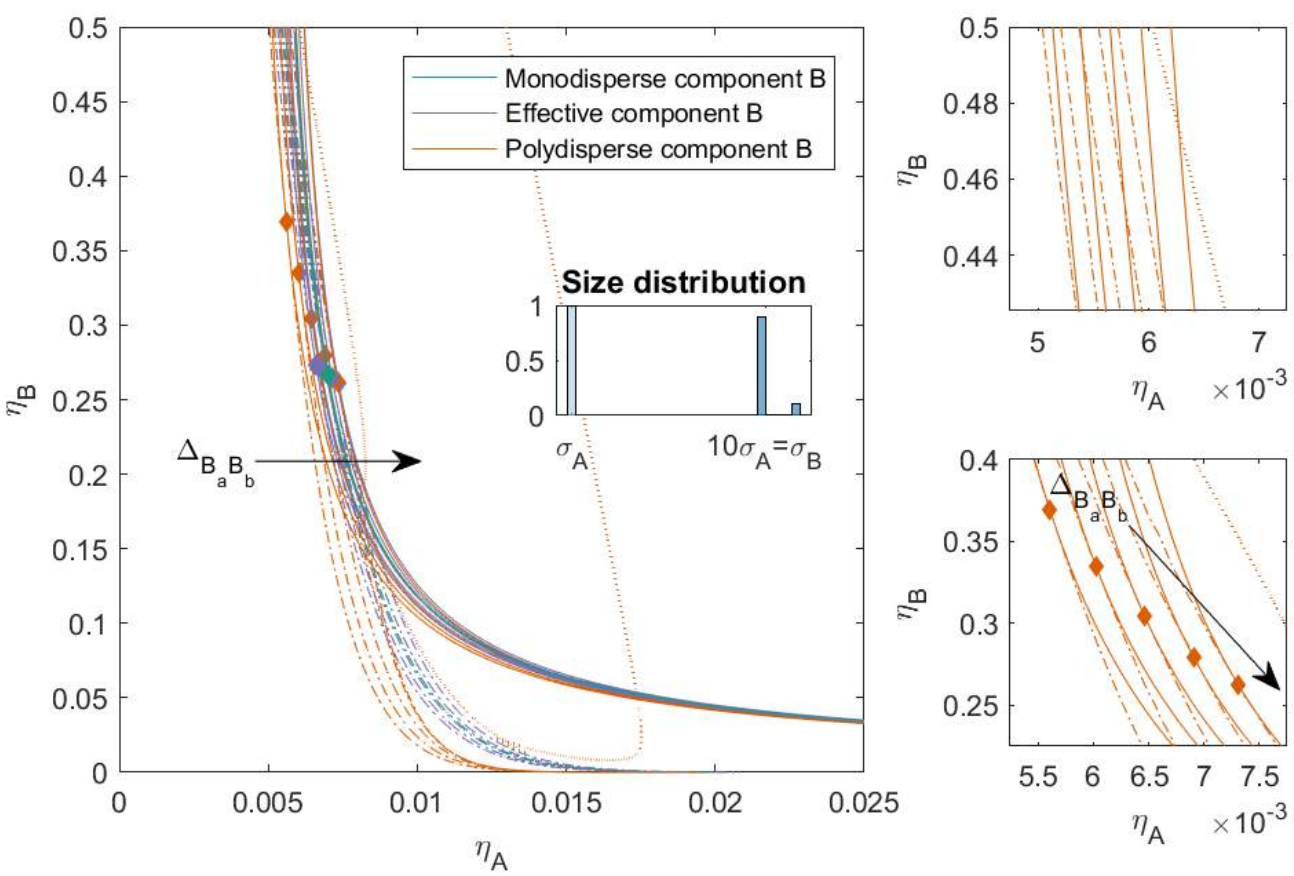

(b) Right skewed

Figure 3.6: Phase diagram for binary (component $A$ and $B$ ) non-additive hard sphere mixture with size ratio $q=\sigma_{A} / \sigma_{B}=1 / 10$, component $A$ is monodisperse, component $B$ is polydisperse $(P D=4.80)$, plotted as a function of the partial packing fractions, $\eta_{A}$ and $\eta_{B}$. The interaction between components $A$ and $B$ is additive, the interaction between the sub-components $B$ is non-additive, the non-additivity parameter $\Delta_{B_{a} B_{b}}$ was varied from -0.1 to 0.1 with a step size of 0.05 (the arrow indicates increasing $\Delta_{B_{a} B_{b}}$ ). The spinodal (solid line) and binodal (dashed line) meet each other at the plait point (diamond), the three phase boundary is indicated with a dotted line. 
compared to the right-skewed mixtures. Next to that, these left-skewed mixtures also show a larger bend in the two-phase boundary towards the y-axis for $\Delta_{B_{a} B_{b}}>0$. The mixture in Figure 3.6a with $\Delta_{B_{a} B_{b}}=0.05$ does not have a three phase boundary, even though mixtures with these sizes can phase separate into three phases: the distribution of the sub-components makes these concentrations unattainable in the range of concentrations we focus on.

For the right-skewed mixtures, we see that the three-phase boundary for mixtures with $\Delta_{B_{a} B_{b}}=0.10$ comes very close to the two-phase boundary and for mixtures with $\Delta_{B_{a} B_{b}}=0.05$ the three phase boundary shows a bend back towards lower concentrations of $A$ at low concentrations of $B$. This is due to the shape of the three-phase surface and can also be seen on a small level in the mixture $\Delta_{B_{a} B_{b}}=0.10$ in Figure 3.3.

Also Bellier-Castella et al. (2000) found the possibility of three phase separation for polydisperse components. According to them, the transition between the two phase and three phase region proceeds via a second critical point. This second critical point is polydispersity induced.

\subsubsection{Mixtures with non-additivity between sub-components of $B$ $\left(\Delta_{B_{a} B_{b}}\right)$, and between $A$ and $B\left(\Delta_{A B}\right)$}

In Figure 3.7 we plot the phase diagram for mixtures with varying $\Delta_{B_{a} B_{b}}$, with a size ratio between component $A$ and $B$ of $q=1 / 3$, and a non-additive interaction between $A$ and $B \Delta_{A B}=0.075$. This is in fact a combination of the case in Section 3.3.1 and Section 3.3.2 at lower size ratio between $A$ and $B$. The polydisperisty of $B$ is 4.00 (for mixtures with more variety in $P D$ and $\Delta_{A B}$ we refer to supplementary material). The phase diagram of these mixtures shows a lot of similarities with the phase diagram of the mixtures in Figure 3.3 though at different $\eta_{A}$ due to the different size ratio. Since the mixtures in Figure $\mathbf{3 . 3}$ have the same $P D$, we conclude that the three-phase boundary position and shape is largely dependent on the interaction between the sub-components of $B$. The interaction between the sub-components is determined by both the $P D$ and the non-additivity parameter $\Delta_{B_{a} B_{b}}$.

\subsubsection{Fractionation}

When a parent mixture demixes into two or more phases, each component (and also their subcomponents) in the mixture will find its preferential phase in order to minimize the Helmholtz free energy of the system. This leads each phase to be enriched in one of the components, whilst being depleted by the other component(s). The other component(s) are then present 


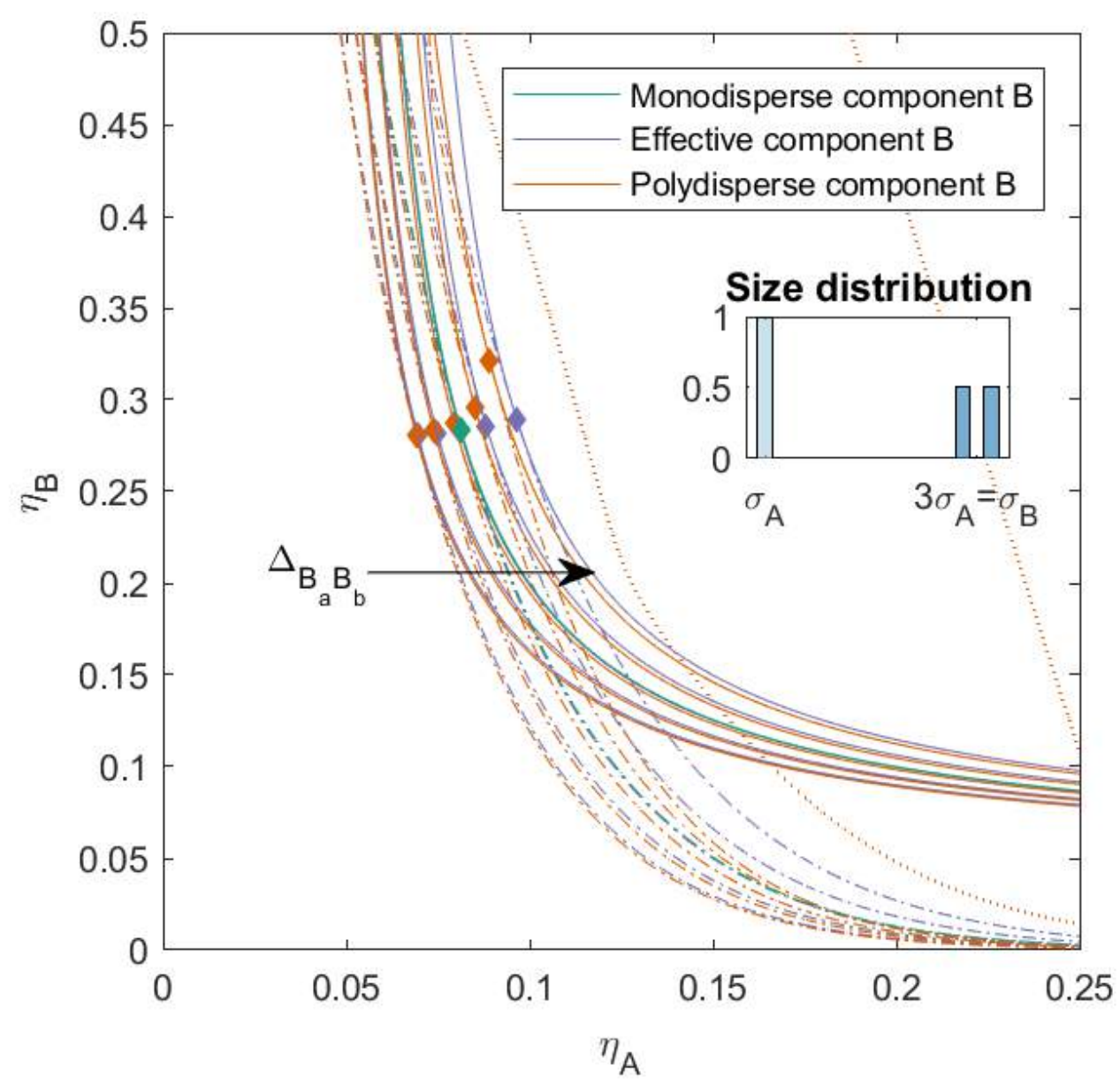

Figure 3.7: Phase diagram for binary (component $A$ and $B$ ) non-additive hard sphere mixture with size ratio $q=\sigma_{A} / \sigma_{B}=1 / 3$, component $A$ is monodisperse, component $B$ is polydisperse $(P D=4.00)$, plotted as a function of the partial packing fractions, $\eta_{A}$ and $\eta_{B}$. The interaction between components $A$ and $B$ is non-additive with a non-additivity parameter $\Delta_{A B}=0.075$, the interaction between the sub-components $B$ is non-additive, the non-additivity parameter $\Delta_{B_{a} B_{b}}$ was varied from -0.1 to 0.1 with a step size of 0.05 (the arrow indicates increasing $\Delta_{B_{a} B_{b}}$ ). The spinodal (solid line) and binodal (dashed line) meet each other at the plait point (diamond), the three phase boundary is indicated with a dotted line. 


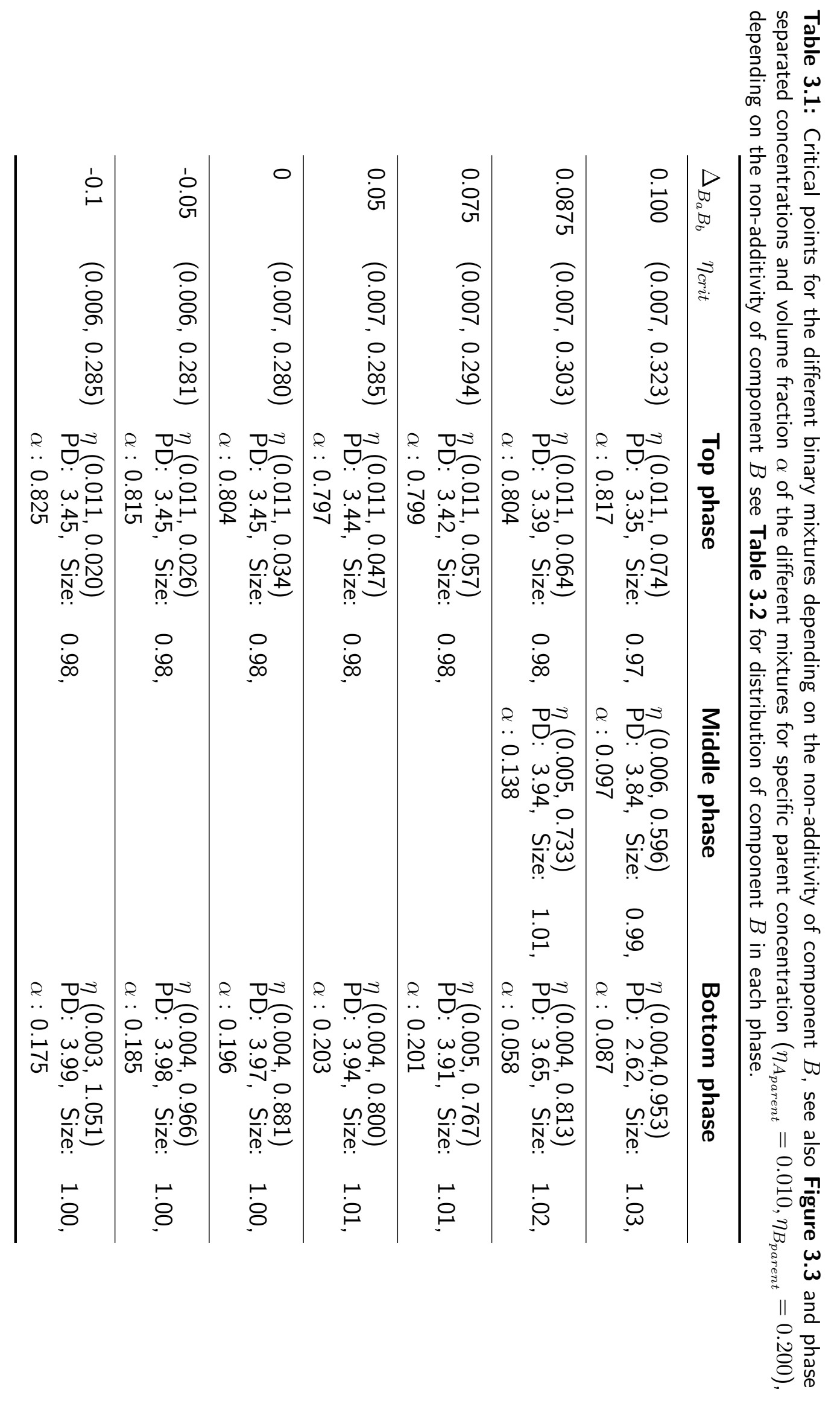


only at low volume fractions. We investigated the phase separation for the mixtures in Section 3.3.2 for a specific parent mixture $\left(\eta_{A_{\text {parent }}}=0.010, \eta_{B_{\text {parent }}}=0.200\right)$ in terms of the volume fraction of both components in each phase, the degree of polydispersity of component $B$, the average size of component $B$ in the child phases compared to the average size of component $B$ in the parent phase and the volume fraction of the phases $(\alpha)$ see Table 3.1 for the mixtures from Figure 3.3 (mixtures with $P D=4.00$ ). For other mixtures we refer to the supplementary materials. The composition histograms for each phase are given in Table $\mathbf{3 . 2}$ for the same mixture, for other mixtures we refer to the supplementary materials. Since at this parent concentration the mixture with non-additivity parameter $\Delta_{B_{a} B_{b}}=0.10$ separates into three phases, we have also calculated the child phases for mixtures with between $\Delta_{B_{a} B_{b}}=0.10$ and $\Delta_{B_{a} B_{b}}=0.05$ to investigate the behavior of the sub-components $B$ depending on the non-additivity.

For all mixtures, the top phase, which is also the largest phase in volume, is enriched in component $A$. The volume fraction of the top phase is dependent on the non-additive interaction between the sub-components of $B$. It increases with both more compatibility between the sub-components as well as less compatibility, with a minimum volume fraction at $\Delta_{B_{a} B_{b}}=0.05$. We find this dependence in volume fraction on the non-additivity parameter $\Delta_{B_{a} B_{b}}$ also for the other mixtures, however the minimum volume fraction is at different $\Delta_{B_{a} B_{b}}$ depending on the sizes and the ratio of the sub-components $a$ and $b$ of $B$. For the mixtures $\left(\Delta_{B_{a} B_{b}}>0.075\right)$ that phase separate into three phases at this parent mixture concentration, we conclude that it is mostly the bottom phase that demixes into an additional phase (the middle phase). The bottom phase is enriched in the largest sub-component of $B$, while the top phase (and middle phase to a lesser extent) is enriched in the smaller sub-component of $B$. We see this behavior also for the other mixtures with different composition of $B$.

The fractionation of the sub-components of $B$ is dependent on the non-additivity parameter $\Delta_{B_{a} B_{b}}$. When $\Delta_{B_{a} B_{b}}<0$ the sub-components $a$ and $b$ are more compatible with each other and this leads to less fractionation, as can be seen in Table 3.2, while on the other hand $\Delta_{B_{a} B_{b}}>0$ the sub-components are less compatible with each other and more fractionation occurs, even leading to additional phase separation at higher $\Delta_{B_{a} B_{b}}$. This is something we see also for the other mixtures (see supplementary material).

\subsection{Conclusion}

We find that when the compatibility between component $A$ and $B$ is decreased, the phase diagram (the critical point, phase boundary and spinodal) shifts towards lower volume 
Table 3.2: Phase separation of different mixtures and fractionation of component $B$ for specific parent distribution $\left(\eta_{A_{\text {parent }}}=0.010, \eta_{B_{\text {parent }}}=0.200\right)$, depending on the non-additivity of component $B$, see also Figure 3.3.

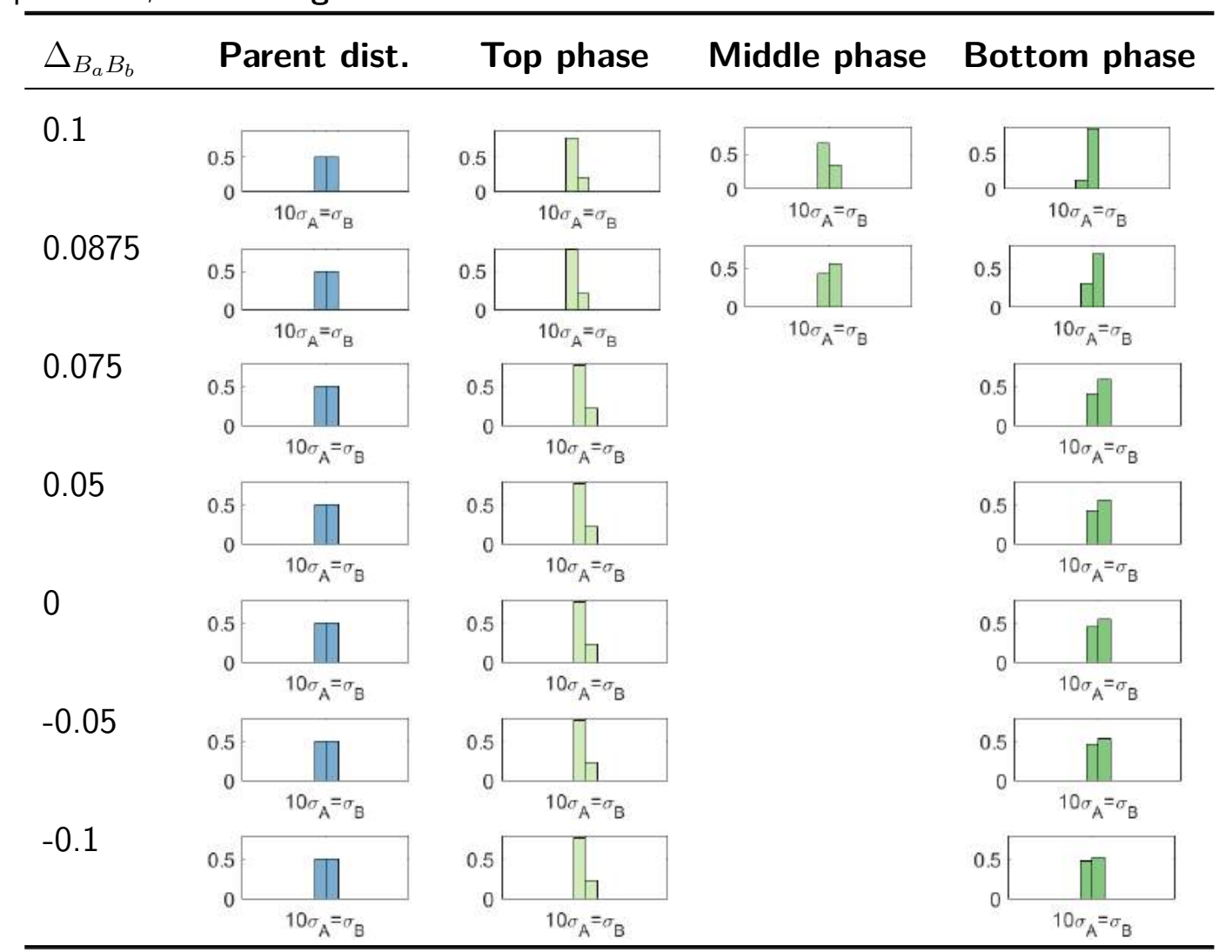

fractions. This is in line with literature on the phase behavior of NAHS binary monodisperse mixtures. The interaction between $A$ and $B$ is driven by the size ratio $(q)$ between $A$ and $B$ and the non-additivity parameter $\Delta_{A B}$.

When the compatibility between the sub-components of the polydisperse component $B$ is altered, the phase diagram changes more drastically. When the compatibility between the sub-components is decreased, the mixture can demix into three phases each enriched in one of the (sub)components of the parent mixture. The shape and position of the three phase boundary is mainly dependent on the interactions between the sub-components of $B$. This means that it is dependent on the non-additivity parameter $\left(\Delta_{B_{a} B_{b}}\right)$ as well as the size ratios and distribution of the sub-components (the degree of polydispersity $P D$ ). Next to that, depending on the size ratios and distribution of the sub-components we see also that the the binodal and spinodal bend towards the $y$-axis for higher volume fractions of $B$ when $\Delta_{B_{a} B_{b}}$ increases. For the mixtures with a more pronounced bend in the phase boundary and spinodal, we find that the critical point shifts to volume fractions $\eta_{B_{c r i t}}>0.5$. This behavior is driven to a large extent by the the non-additivity parameter $\left(\Delta_{B_{a} B_{b}}\right)$ as well as the size ratios and distribution of the sub-components (the degree of polydispersity $P D$ ) 
and to a lesser extent by the interaction between $A$ and $B$. When the compatibility between the sub-components is increased, the mixture demixes at slightly lower packing fractions compared to the HS mixture. The fractionation of the polydisperse sub-components of $B$ is also dependent on the non-additivity parameter $\Delta_{B_{a} B_{b}}$. Less fractionation occurs when $\Delta_{B_{a} B_{b}}<0$, more fractionation occurs when $\Delta_{B_{a} B_{b}}>0$. At higher $\Delta_{B_{a} B_{b}}$ this can even lead to additional phase separation, creating a third phase.

The virial coefficient approach for polydisperse mixtures allows for the prediction of the phase behavior of polydisperse or impure binary mixtures. Not only does it allow for plotting the phase diagram, it also allows for the calculation of the composition and fractionation of each component in each phase. 


\subsection{Bibliography}

B. L. Beegle, M. Modell, and R. C. Reid. Thermodynamic stability criterion for pure substances and mixtures. AIChE Journal, 20(6):1200-1206, 1974. ISSN 0001-1541. doi: 10.1002/aic.690200621.

L. Bellier-Castella, H. Xu, and M. Baus. Phase diagrams of polydisperse van der Waals fluids. Journal of Chemical Physics, 113(18):8337-8347, 2000. ISSN 00219606. doi: 10.1063/1.1316007.

T. Biben and J.-P. Hansen. Spinodal instability of suspensions of large spheres in a fluid of small spheres. Journal of Physics: Condensed Matter, 3(42):F65-F72, 1991. ISSN 0953-8984. doi: 10.1088/0953-8984/3/42/006.

T. Biben and J.-P. Hansen. Osmotic depletion, non-additivity and phase separation. Physica A: Statistical Mechanics and its Applications, 235(1-2):142-148, 1997. ISSN 03784371. doi: 10.1016/S0378-4371(96)00335-4.

M. Dijkstra. Phase behavior of nonadditive hard-sphere mixtures. Physical Review E Statistical Physics, Plasmas, Fluids, and Related Interdisciplinary Topics, 58(6):7523-7528, 1998. ISSN 1063651X. doi: 10.1103/PhysRevE.58.7523.

M. Dijkstra, R. van Roij, and R. Evans. Phase diagram of highly asymmetric binary hardsphere mixtures. Physical Review E - Statistical Physics, Plasmas, Fluids, and Related Interdisciplinary Topics, 59(5):5744-5771, 1999. ISSN 1063651X. doi: 10.1103/PhysRevE.59.5744.

M. W. Edelman, E. van der Linden, E. de Hoog, and R. H. Tromp. Compatibility of Gelatin and Dextran in Aqueous Solution. Biomacromolecules, 2(4):1148-1154, 2001. ISSN 1525-7797. doi: 10.1021/bm015545f.

C. Ersch, E. van der Linden, A. Martin, and P. Venema. Interactions in protein mixtures. Part II: A virial approach to predict phase behavior. Food Hydrocolloids, 52:991-1002, 2016. doi: 10.1016/j.foodhyd.2015.07.021.

R. A. Heidemann. The criteria for thermodynamic stability. AIChE Journal, 21(4):824-826, 1975. ISSN 0001-1541. doi: 10.1002/aic.690210433.

R. A. Heidemann. The Classical Theory of Critical Points. In Supercritical Fluids, pages 39-64. Springer Netherlands, 1994. doi: 10.1007/978-94-015-8295-7_2. 
R. A. Heidemann and A. M. Khalil. The calculation of critical points. A/ChE Journal, 26 (5):769-779, 1980. ISSN 15475905. doi: 10.1002/aic.690260510.

T. L. Hill. An Introduction to Statistical Thermodynamics. Dover Publications, New York, 1986. ISBN 9780486652429.

P. Hopkins and M. Schmidt. Binary non-additive hard sphere mixtures: Fluid demixing, asymptotic decay of correlations and free fluid interfaces. Journal of Physics Condensed Matter, 22(32), 2010. ISSN 09538984. doi: 10.1088/0953-8984/22/32/325108.

V. Kontogiorgos, S. M. Tosh, and P. J. Wood. Phase behaviour of high molecular weight oat $\beta$-glucan/whey protein isolate binary mixtures. Food Hydrocolloids, 23(3):949-956, 2009. ISSN 0268005X. doi: 10.1016/j.foodhyd.2008.07.005.

H. N. Lekkerkerker and R. Tuinier. Colloids and the Depletion Interaction, volume 833. Springer, 2011. ISBN 978-94-007-1222-5. doi: 10.1007/978-94-007-1223-2.

P. Paricaud. Phase equilibria in polydisperse nonadditive hard-sphere systems. Physical Review E, 78(2):021202, 2008. ISSN 1539-3755. doi: 10.1103/PhysRevE.78.021202.

M. Piech and J. Y. Walz. Effect of Polydispersity and Charge Heterogeneity on the Depletion Interaction in Colloidal Systems. Journal of Colloid and Interface Science, 225(1):134-146, 2000. ISSN 00219797. doi: 10.1006/jcis.2000.6755.

R. C. Reid and B. L. Beegle. Critical point criteria in legendre transform notation. AIChE Journal, 23(5):726-732, 1977. ISSN 15475905. doi: 10.1002/aic.690230515.

R. Roth, R. Evans, and A. A. Louis. Theory of asymmetric nonadditive binary hard-sphere mixtures. Physical Review E - Statistical Physics, Plasmas, Fluids, and Related Interdisciplinary Topics, 64(5):13, 2001. ISSN 1063651X. doi: 10.1103/PhysRevE.64.051202.

W. F. Sager. Systematic study on the influence of impurities on the phase behavior of sodium bis(2-ethylhexyl) sulfosuccinate microemulsions. Langmuir, 14(22):6385-6395, 1998. ISSN 07437463. doi: 10.1021/la9709608.

R. P. Sear and D. Frenkel. Phase behavior of colloid plus polydisperse polymer mixtures. Physical Review E - Statistical Physics, Plasmas, Fluids, and Related Interdisciplinary Topics, 55(2):1677-1681, 1997. ISSN 1063651X. doi: 10.1103/PhysRevE.55.1677.

P. Sillren and J. P. Hansen. On the critical non-additivity driving segregation of asymmetric binary hard sphere fluids. Molecular Physics, 108(1):97-104, 2010. ISSN 13623028. doi: $10.1080 / 00268970903514561$. 
M. A. Solokhin, A. V. Solokhin, and V. S. Timofeev. Phase-equilibrium stability criterion in terms of the eigenvalues of the Hessian matrix of the Gibbs potential. Theoretical Foundations of Chemical Engineering, 36(5):444-446, 2002. ISSN 00405795. doi: 10.1023/A:1020617610644.

L. Sturtewagen and E. van der Linden. Effect of polydispersity on the phase behavior of additive hard spheres in solution, part I. Manuscript submitted, 2019. URL http://arxiv.org/abs/1912.03143. 


\section{A Figures}

\section{A.1 Variation in $\Delta_{A B}$ between component $\mathbf{A}$ and $\mathbf{B}$}

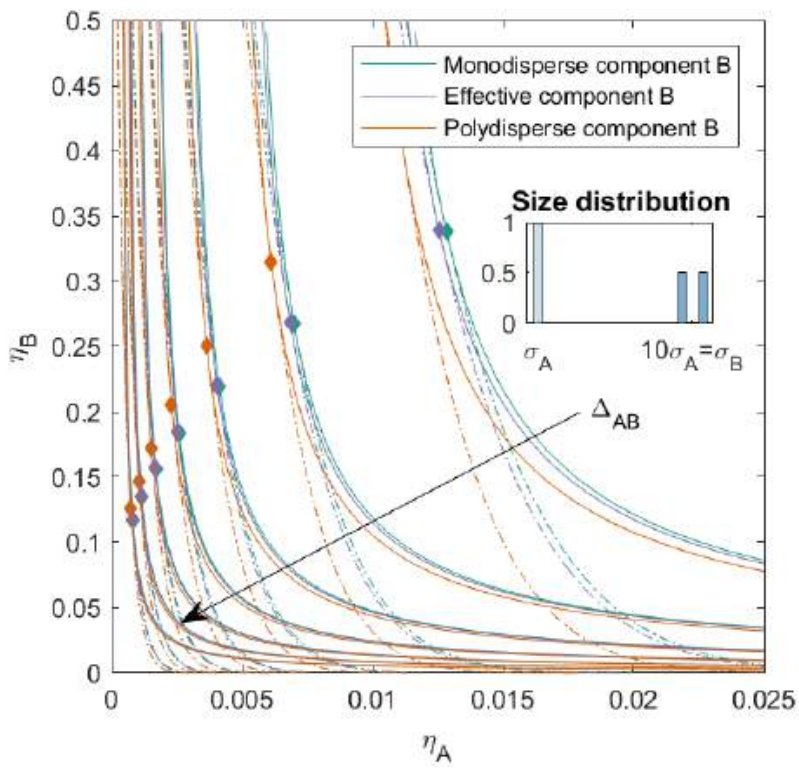

(a) $P D=8.00$

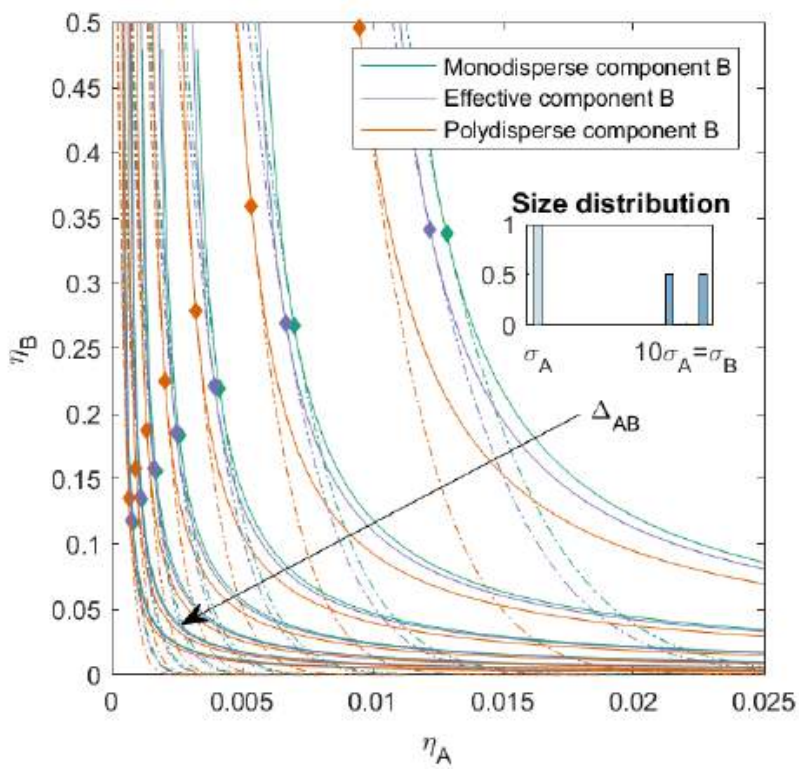

(b) $P D=12.00$

Figure 3.A.1: Phase diagram for binary (component $A$ and $B$ ) non-additive hard sphere mixture with size ratio $q=\sigma_{A} / \sigma_{B}=1 / 10$, component $A$ is monodisperse, component $B$ is polydisperse, plotted as a function of the partial packing fractions, $\eta_{A}$ and $\eta_{B}$. The interaction between components $A$ and $B$ is non-additive, the non-additivity parameter $\Delta_{A B}$ was varied from -0.1 to 0.5 with a step size of 0.1 (the arrow indicates increasing $\Delta_{A B}$ ). The interaction between the sub-components $B$ is additive. The spinodal (solid line) and binodal (dashed line) meet each other at the critical point (diamond). 


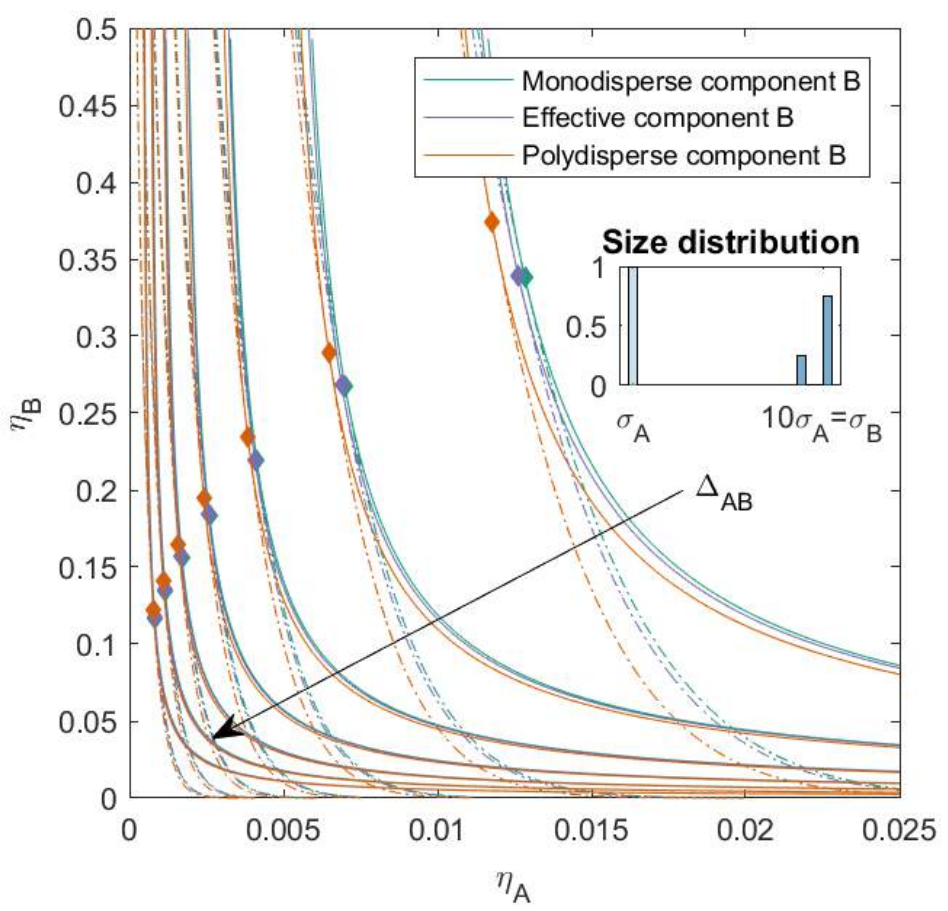

(a) Left skewed

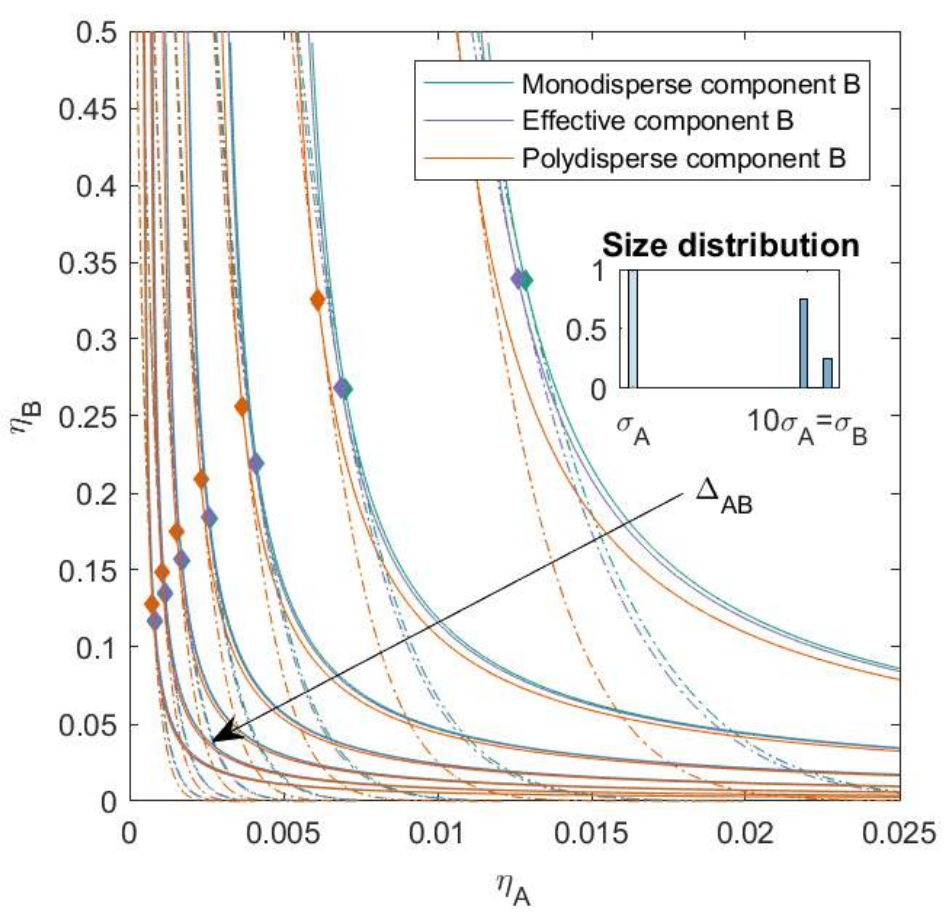

(b) Right skewed

Figure 3.A.2: Phase diagram for binary (component $A$ and $B$ ) non-additive hard sphere mixture with size ratio $q=\sigma_{A} / \sigma_{B}=1 / 10$, component $A$ is monodisperse, component $B$ is polydisperse $(P D=6.93)$, plotted as a function of the partial packing fractions, $\eta_{A}$ and $\eta_{B}$. The interaction between components $A$ and $B$ is non-additive, the non-additivity parameter $\Delta_{A B}$ was varied from -0.1 to 0.5 with a step size of 0.1 (the arrow indicates increasing $\Delta_{A B}$ ). The interaction between the sub-components $B$ is additive. The spinodal (solid line) and binodal (dashed line) meet each other at the critical point (diamond). 


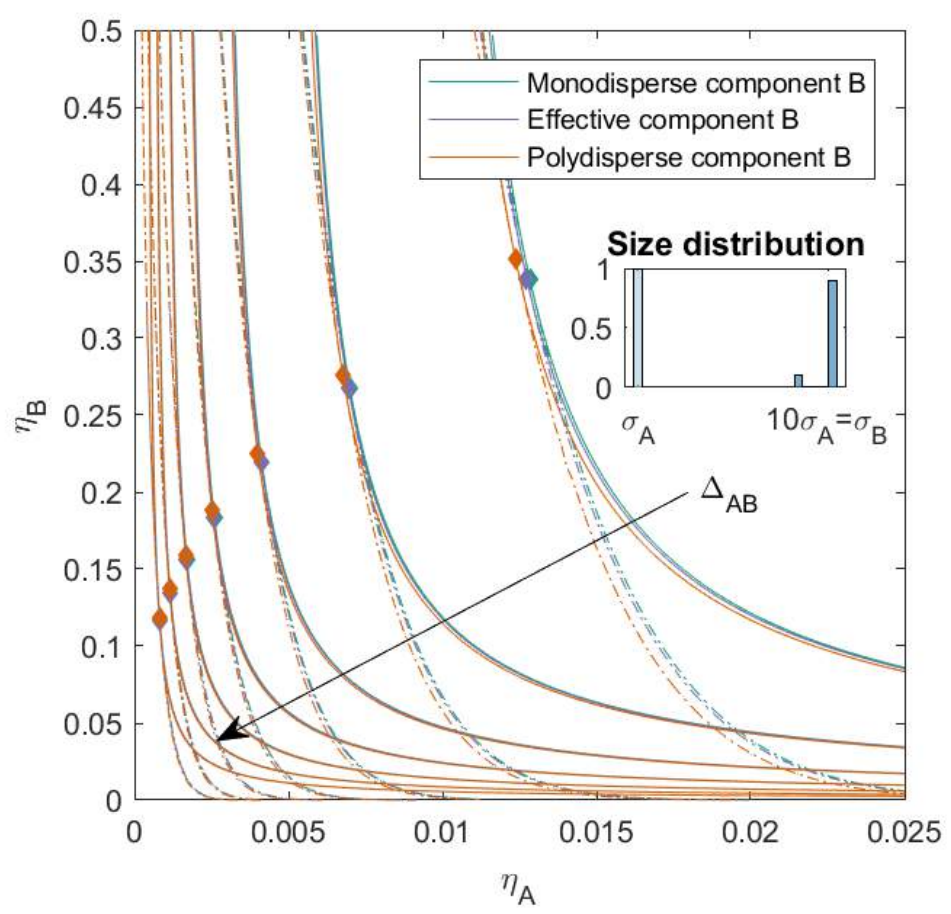

(a) Left skewed

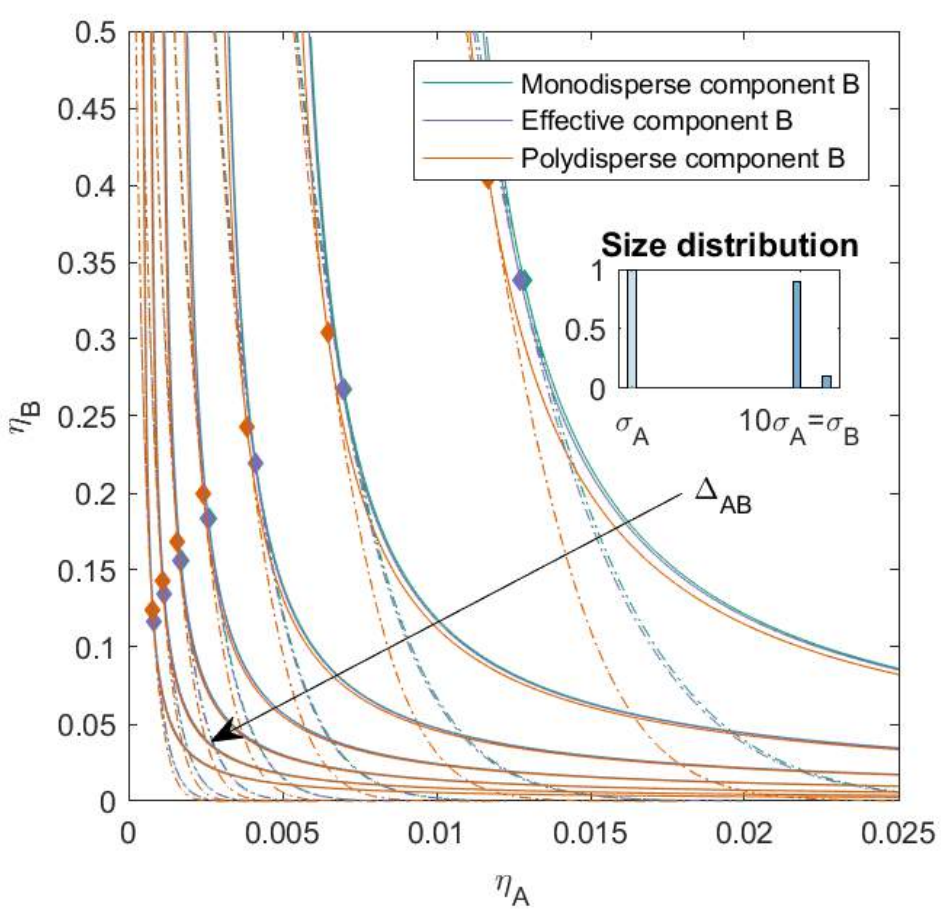

(b) Right skewed

Figure 3.A.3: Phase diagram for binary (component $A$ and $B$ ) non-additive hard sphere mixture with size ratio $q=\sigma_{A} / \sigma_{B}=1 / 10$, component $A$ is monodisperse, component $B$ is polydisperse $(P D=4.80)$, plotted as a function of the partial packing fractions, $\eta_{A}$ and $\eta_{B}$. The interaction between components $A$ and $B$ is non-additive, the non-additivity parameter $\Delta_{A B}$ was varied from -0.1 to 0.5 with a step size of 0.1 (the arrow indicates increasing $\Delta_{A B}$ ). The interaction between the sub-components $B$ is additive. The spinodal (solid line) and binodal (dashed line) meet each other at the critical point (diamond). 


\section{A.2 Variation in $\Delta_{B_{a} B_{b}}$ between sub-components $\mathbf{B}$ at lower size ratio $(q)$ between component $\mathbf{A}$ and $\mathbf{B}$ for different $\Delta_{A B}$}

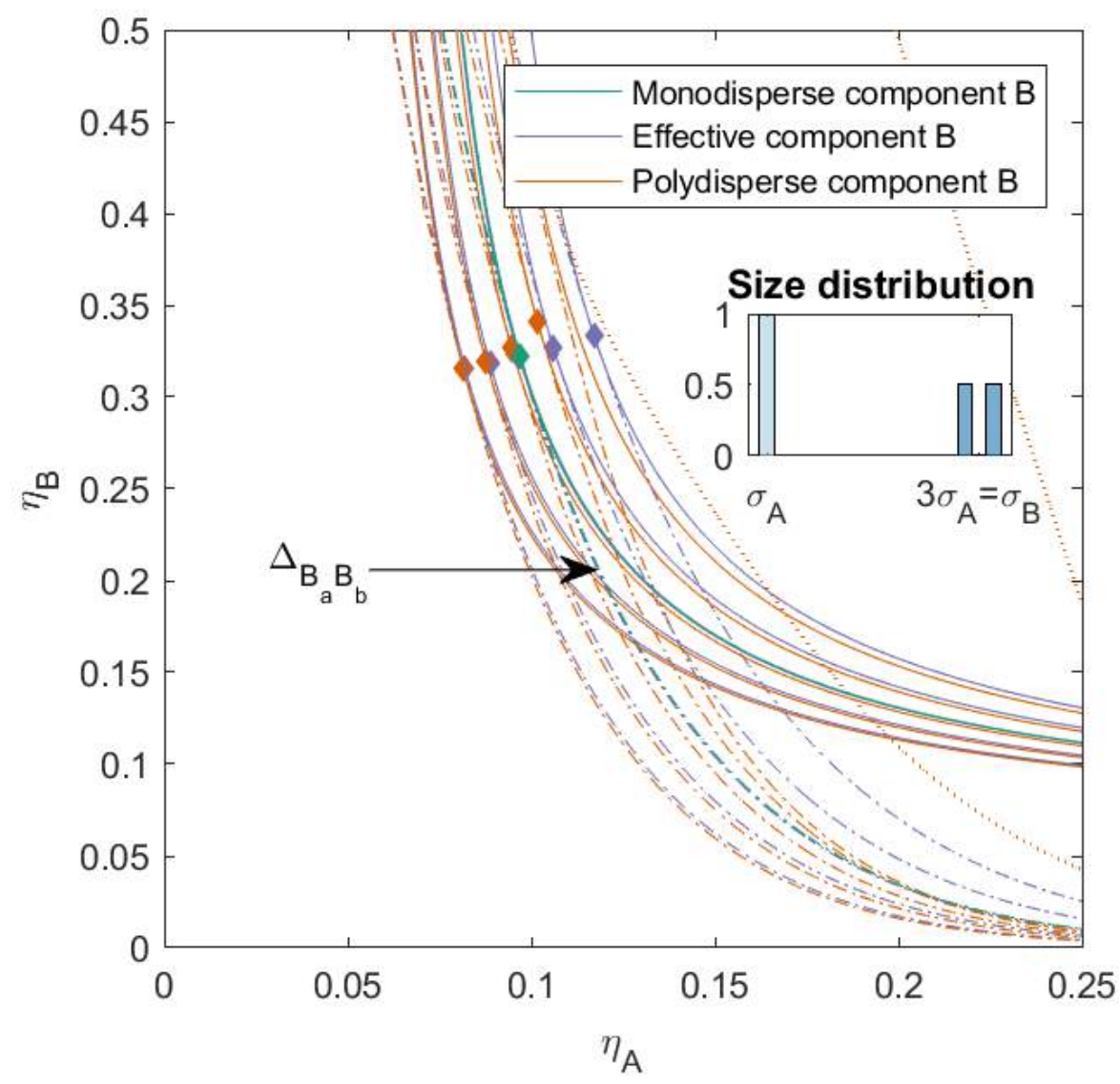

Figure 3.A.4: Phase diagram for binary (component $A$ and $B$ ) non-additive hard sphere mixture with size ratio $q=\sigma_{A} / \sigma_{B}=1 / 3$, component $A$ is monodisperse, component $B$ is polydisperse $(P D=4.00)$, plotted as a function of the partial packing fractions, $\eta_{A}$ and $\eta_{B}$. The interaction between components $A$ and $B$ is non-additive with a non-additivity parameter $\Delta_{A B}=0.05$, the interaction between the sub-components $B$ is non-additive, the non-additivity parameter $\Delta_{B_{a} B_{b}}$ was varied from -0.1 to 0.1 with a step size of 0.05 (the arrow indicates increasing $\Delta_{B_{a} B_{b}}$ ). The spinodal (solid line) and binodal (dashed line) meet each other at the plait point (diamond), the three phase boundary is indicated with a dotted line. 


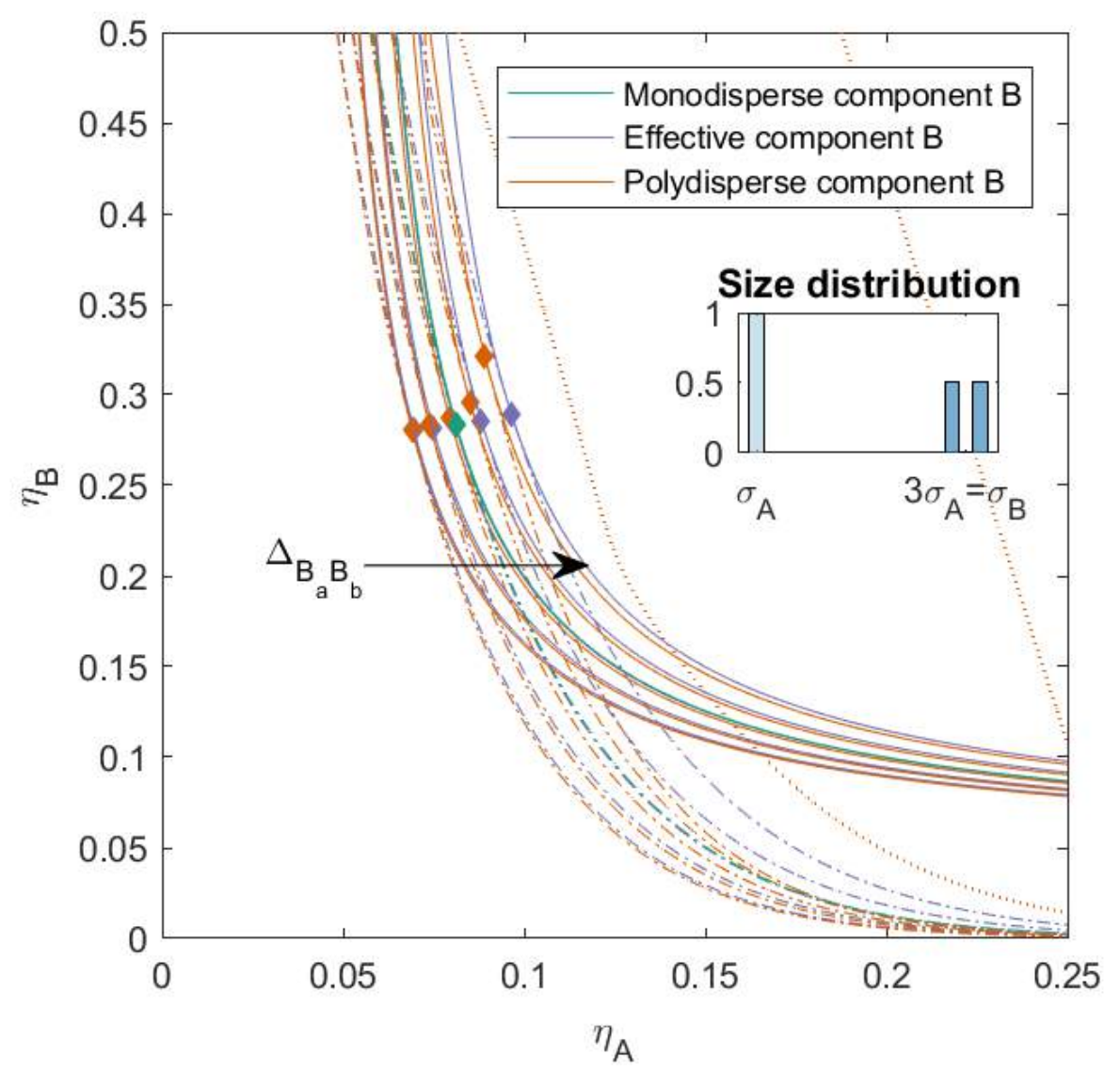

Figure 3.A.5: Phase diagram for binary (component $A$ and $B$ ) non-additive hard sphere mixture with size ratio $q=\sigma_{A} / \sigma_{B}=1 / 3$, component $A$ is monodisperse, component $B$ is polydisperse $(P D=4.00)$, plotted as a function of the partial packing fractions, $\eta_{A}$ and $\eta_{B}$. The interaction between components $A$ and $B$ is non-additive with a non-additivity parameter $\Delta_{A B}=0.075$, the interaction between the sub-components $B$ is non-additive, the non-additivity parameter $\Delta_{B_{a} B_{b}}$ was varied from -0.1 to 0.1 with a step size of 0.05 (the arrow indicates increasing $\Delta_{B_{a} B_{b}}$ ). The spinodal (solid line) and binodal (dashed line) meet each other at the plait point (diamond), the three phase boundary is indicated with a dotted line. 


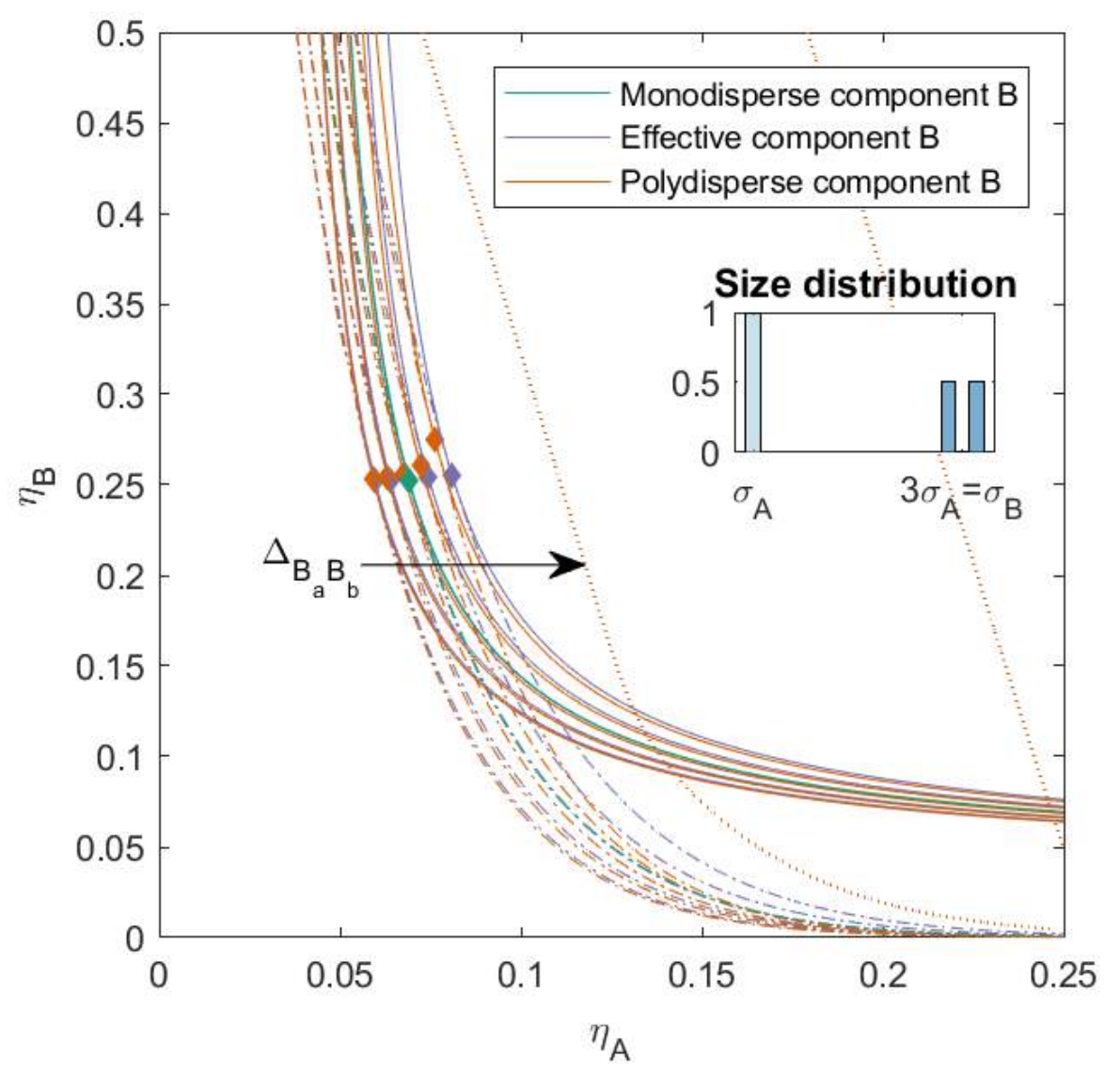

Figure 3.A.6: Phase diagram for binary (component $A$ and $B$ ) non-additive hard sphere mixture with size ratio $q=\sigma_{A} / \sigma_{B}=1 / 3$, component $A$ is monodisperse, component $B$ is polydisperse $(P D=4.00)$, plotted as a function of the partial packing fractions, $\eta_{A}$ and $\eta_{B}$. The interaction between components $A$ and $B$ is non-additive with a non-additivity parameter $\Delta_{A B}=0.10$, the interaction between the sub-components $B$ is non-additive, the non-additivity parameter $\Delta_{B_{a} B_{b}}$ was varied from -0.1 to 0.1 with a step size of 0.05 (the arrow indicates increasing $\Delta_{B_{a} B_{b}}$ ). The spinodal (solid line) and binodal (dashed line) meet each other at the plait point (diamond), the three phase boundary is indicated with a dotted line. 


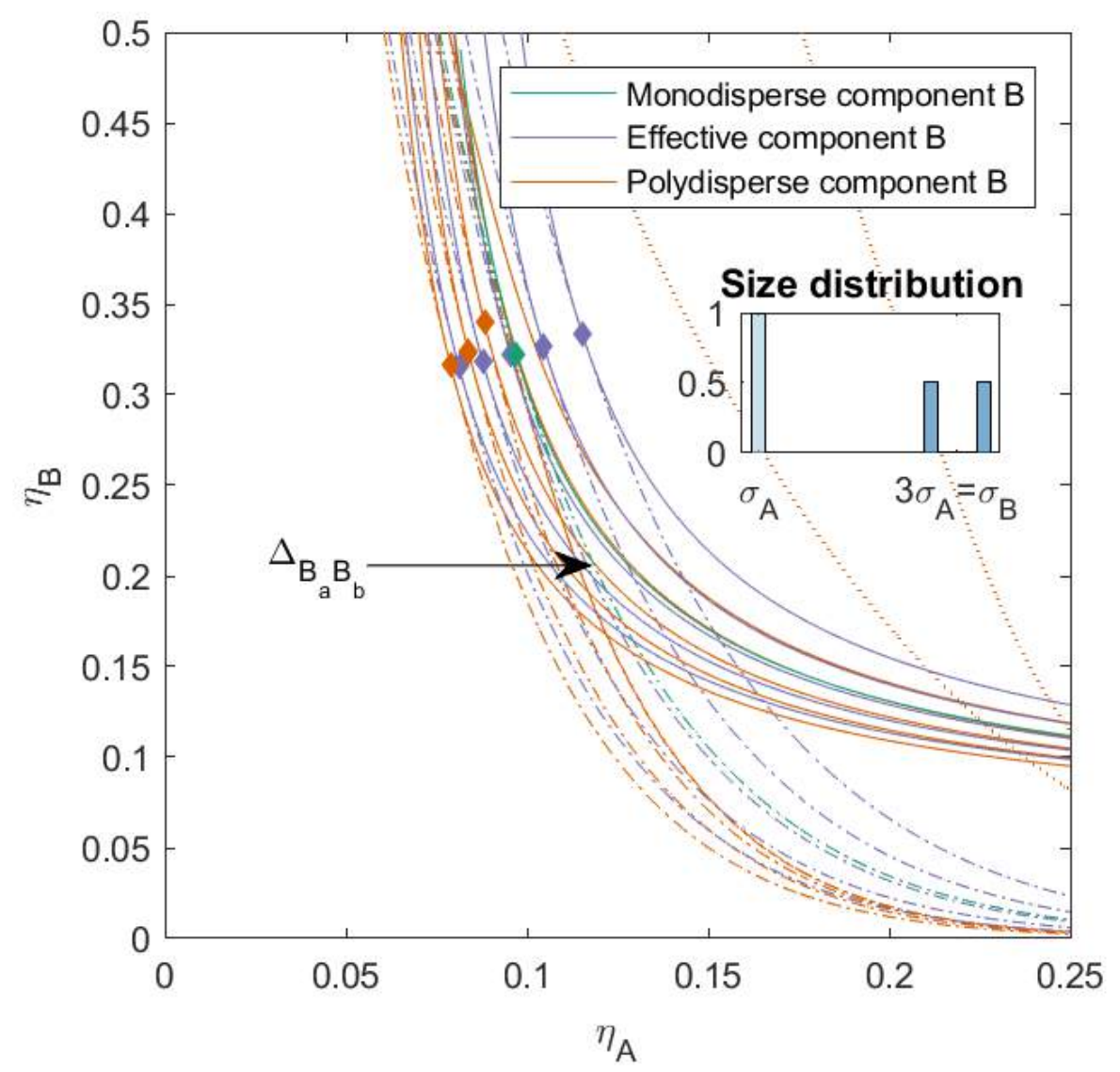

Figure 3.A.7: Phase diagram for binary (component $A$ and $B$ ) non-additive hard sphere mixture with size ratio $q=\sigma_{A} / \sigma_{B}=1 / 3$, component $A$ is monodisperse, component $B$ is polydisperse $(P D=8.00)$, plotted as a function of the partial packing fractions, $\eta_{A}$ and $\eta_{B}$. The interaction between components $A$ and $B$ is non-additive with a non-additivity parameter $\Delta_{A B}=0.05$, the interaction between the sub-components $B$ is non-additive, the non-additivity parameter $\Delta_{B_{a} B_{b}}$ was varied from -0.1 to 0.1 with a step size of 0.05 (the arrow indicates increasing $\Delta_{B_{a} B_{b}}$ ). The spinodal (solid line) and binodal (dashed line) meet each other at the plait point (diamond), the three phase boundary is indicated with a dotted line. 


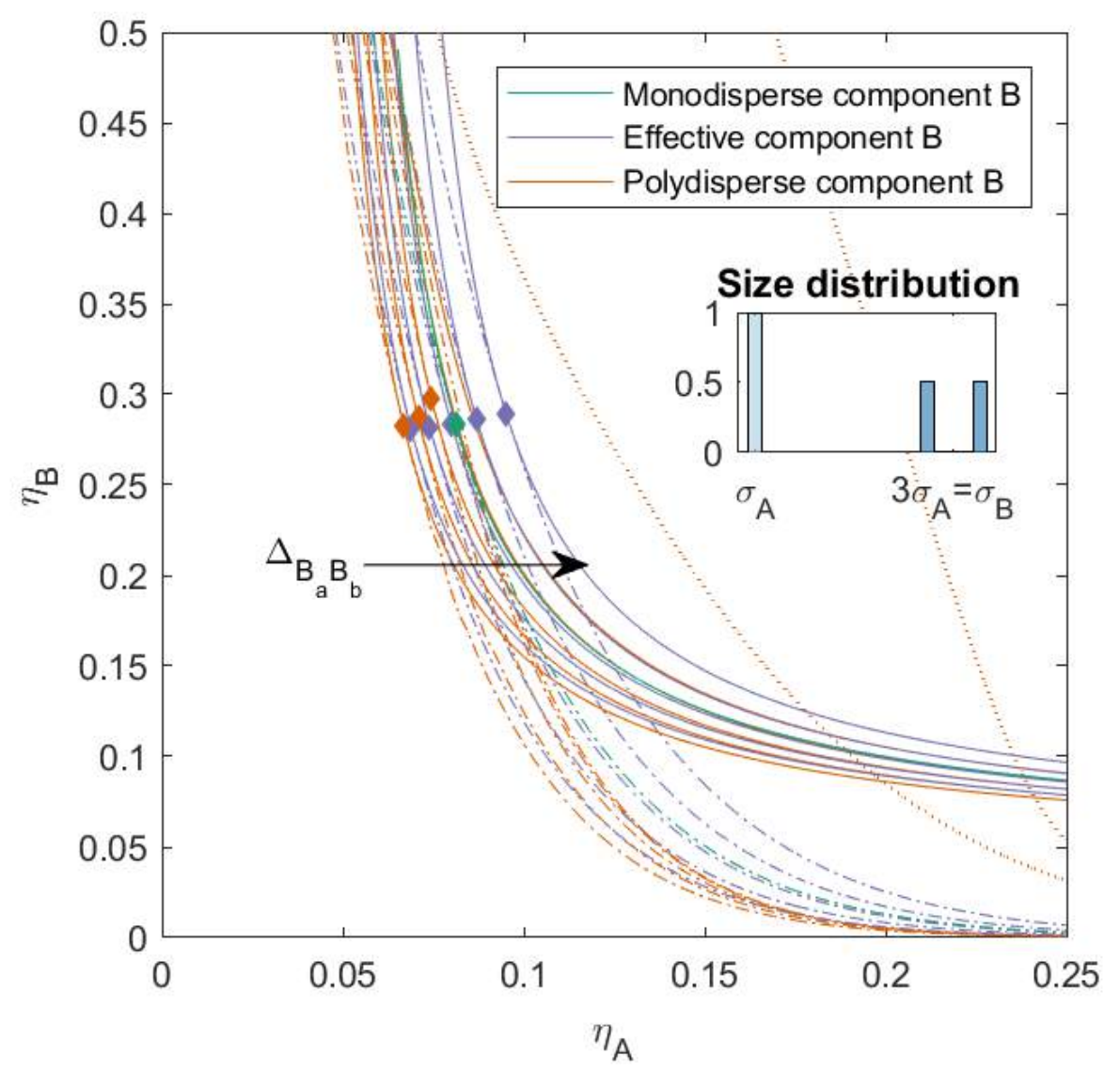

Figure 3.A.8: Phase diagram for binary (component $A$ and $B$ ) non-additive hard sphere mixture with size ratio $q=\sigma_{A} / \sigma_{B}=1 / 3$, component $A$ is monodisperse, component $B$ is polydisperse $(P D=8.00)$, plotted as a function of the partial packing fractions, $\eta_{A}$ and $\eta_{B}$. The interaction between components $A$ and $B$ is non-additive with a non-additivity parameter $\Delta_{A B}=0.075$, the interaction between the sub-components $B$ is non-additive, the non-additivity parameter $\Delta_{B_{a} B_{b}}$ was varied from -0.1 to 0.1 with a step size of 0.05 (the arrow indicates increasing $\Delta_{B_{a} B_{b}}$ ). The spinodal (solid line) and binodal (dashed line) meet each other at the plait point (diamond), the three phase boundary is indicated with a dotted line. 


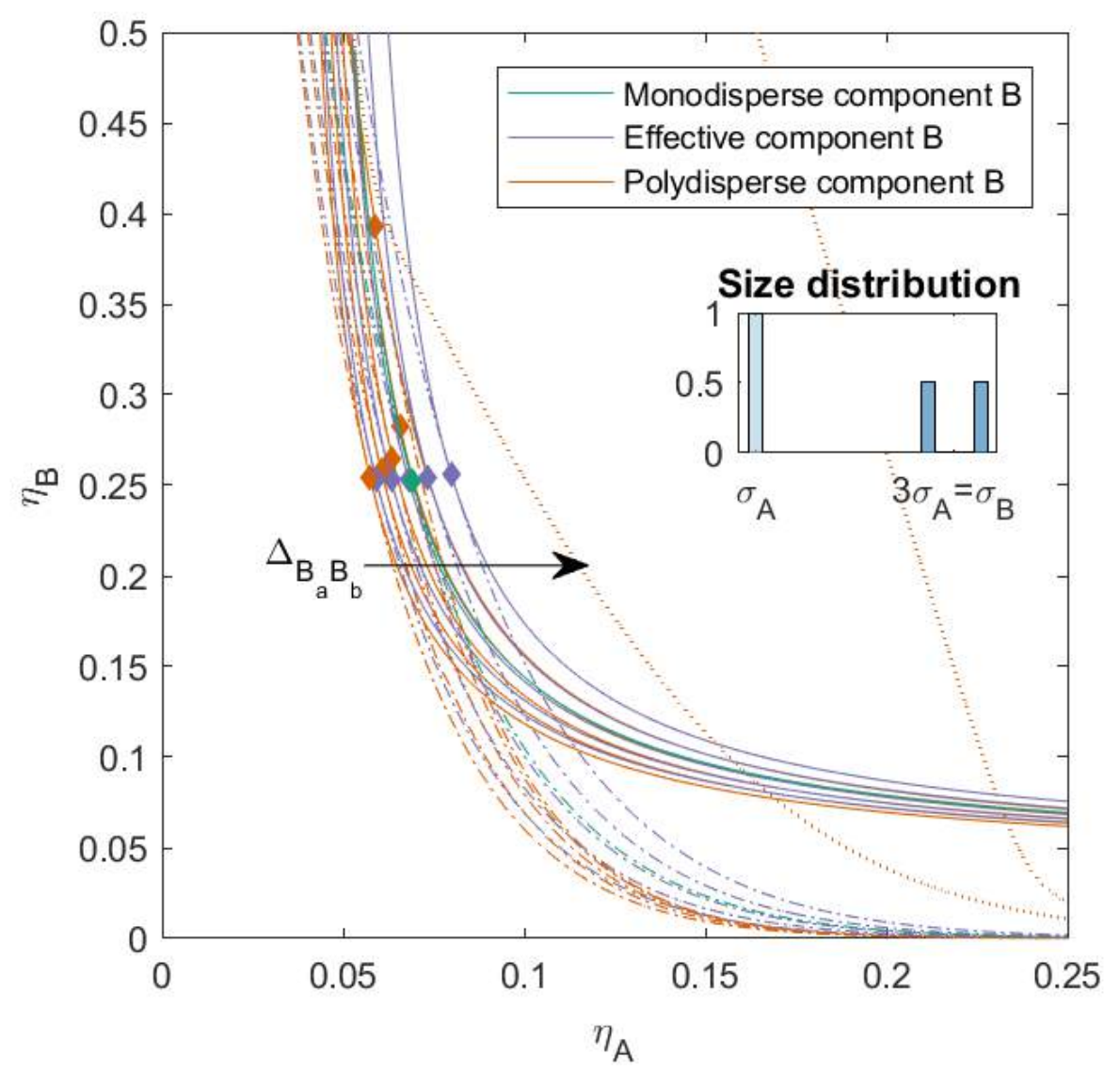

Figure 3.A.9: Phase diagram for binary (component $A$ and $B$ ) non-additive hard sphere mixture with size ratio $q=\sigma_{A} / \sigma_{B}=1 / 3$, component $A$ is monodisperse, component $B$ is polydisperse $(P D=8.00)$, plotted as a function of the partial packing fractions, $\eta_{A}$ and $\eta_{B}$. The interaction between components $A$ and $B$ is non-additive with a non-additivity parameter $\Delta_{A B}=0.1$, the interaction between the sub-components $B$ is non-additive, the non-additivity parameter $\Delta_{B_{a} B_{b}}$ was varied from -0.1 to 0.1 with a step size of 0.05 (the arrow indicates increasing $\Delta_{B_{a} B_{b}}$ ). The spinodal (solid line) and binodal (dashed line) meet each other at the plait point (diamond), the three phase boundary is indicated with a dotted line. 


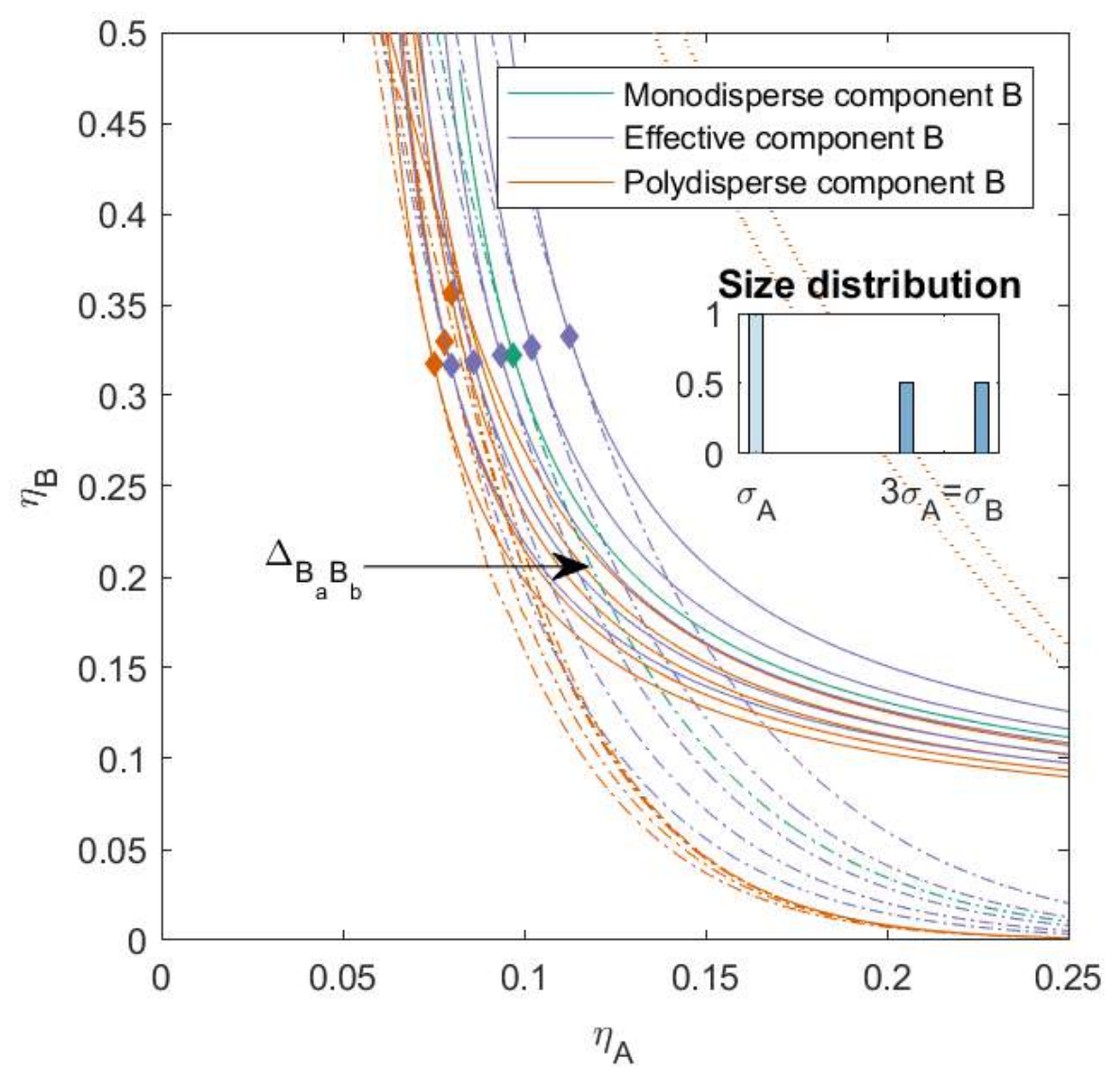

Figure 3.A.10: Phase diagram for binary (component $A$ and $B$ ) non-additive hard sphere mixture with size ratio $q=\sigma_{A} / \sigma_{B}=1 / 3$, component $A$ is monodisperse, component $B$ is polydisperse $(P D=12.00)$, plotted as a function of the partial packing fractions, $\eta_{A}$ and $\eta_{B}$. The interaction between components $A$ and $B$ is non-additive with a non-additivity parameter $\Delta_{A B}=0.05$, the interaction between the sub-components $B$ is non-additive, the non-additivity parameter $\Delta_{B_{a} B_{b}}$ was varied from -0.1 to 0.1 with a step size of 0.05 (the arrow indicates increasing $\Delta_{B_{a} B_{b}}$ ). The spinodal (solid line) and binodal (dashed line) meet each other at the plait point (diamond), the three phase boundary is indicated with a dotted line. 


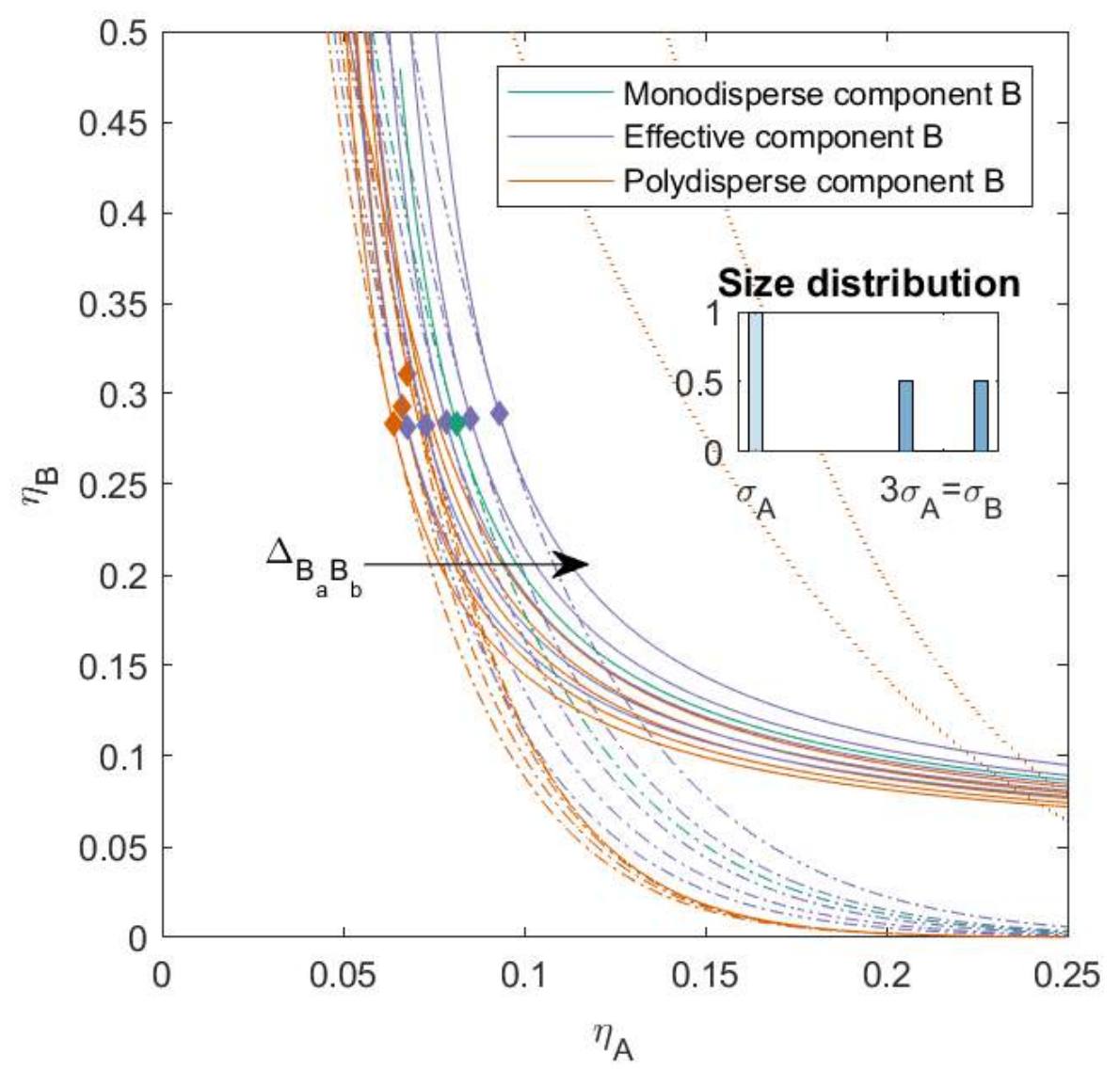

Figure 3.A.11: Phase diagram for binary (component $A$ and $B$ ) non-additive hard sphere mixture with size ratio $q=\sigma_{A} / \sigma_{B}=1 / 3$, component $A$ is monodisperse, component $B$ is polydisperse $(P D=12.00)$, plotted as a function of the partial packing fractions, $\eta_{A}$ and $\eta_{B}$. The interaction between components $A$ and $B$ is non-additive with a non-additivity parameter $\Delta_{A B}=0.075$, the interaction between the sub-components $B$ is non-additive, the non-additivity parameter $\Delta_{B_{a} B_{b}}$ was varied from -0.1 to 0.1 with a step size of 0.05 (the arrow indicates increasing $\Delta_{B_{a} B_{b}}$ ). The spinodal (solid line) and binodal (dashed line) meet each other at the plait point (diamond), the three phase boundary is indicated with a dotted line. 


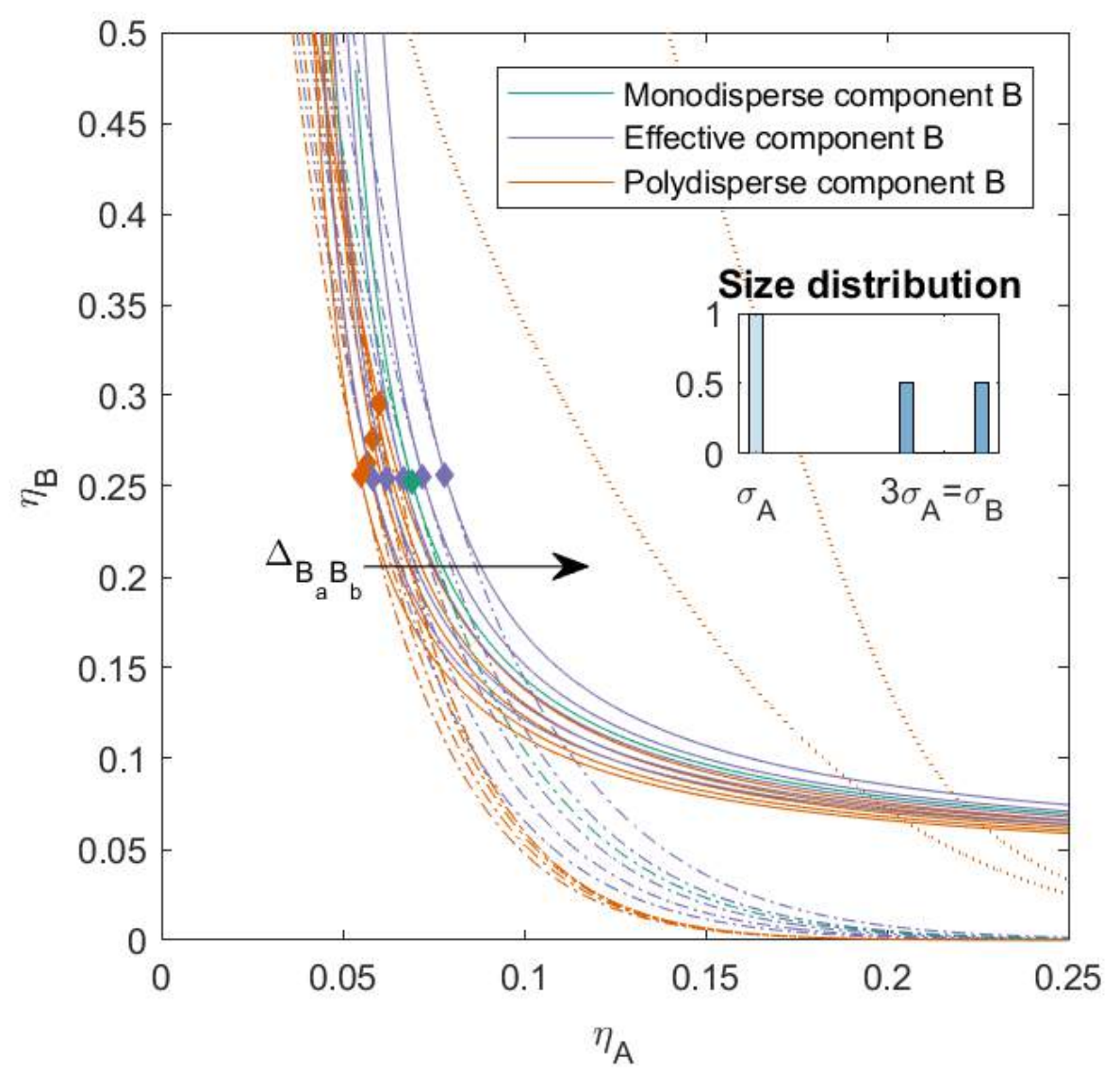

Figure 3.A.12: Phase diagram for binary (component $A$ and $B$ ) non-additive hard sphere mixture with size ratio $q=\sigma_{A} / \sigma_{B}=1 / 3$, component $A$ is monodisperse, component $B$ is polydisperse $(P D=12.00)$, plotted as a function of the partial packing fractions, $\eta_{A}$ and $\eta_{B}$. The interaction between components $A$ and $B$ is non-additive with a non-additivity parameter $\Delta_{A B}=0.1$, the interaction between the sub-components $B$ is non-additive, the non-additivity parameter $\Delta_{B_{a} B_{b}}$ was varied from -0.1 to 0.1 with a step size of 0.05 (the arrow indicates increasing $\Delta_{B_{a} B_{b}}$ ). The spinodal (solid line) and binodal (dashed line) meet each other at the plait point (diamond), the three phase boundary is indicated with a dotted line. 


\section{B Fractionation}

Table 3.B.1: Critical points for the different binary mixtures depending on the non-additivity of component $B$, see also Figure 3.4a and phase separated concentrations and fractionation of the different mixtures for specific parent concentration $\left(\eta_{A_{\text {parent }}}=0.010, \eta_{B_{\text {parent }}}=0.200\right)$, depending on the non-additivity of component $B, P D=8.00$.

\begin{tabular}{|c|c|c|c|}
\hline$\Delta_{B_{a} B_{b}}$ & $\eta_{\text {crit }}$ & Top phase & Bottom phase \\
\hline \multirow[t]{3}{*}{0.100} & - & $\begin{array}{l}\eta(0.011,0.092) \\
\text { PD: } 4.65, \text { Size: } 0.93 \\
\alpha: 0.903\end{array}$ & $\begin{array}{l}\eta(0.003,1.206) \\
\text { PD: } 3.10, \text { Size: } 1.07 \\
\alpha: 0.097\end{array}$ \\
\hline & ${ }^{1}$ & $\begin{array}{r}1 \\
0.5\end{array}$ & $\begin{array}{r}1 \\
0.5\end{array}$ \\
\hline & $10 \sigma_{\mathrm{A}}=\sigma_{\mathrm{B}}$ & $10 \sigma_{\mathrm{A}}=\sigma_{\mathrm{B}}$ & $10 \sigma_{\mathrm{A}}=\sigma_{\mathrm{B}}$ \\
\hline \multirow[t]{3}{*}{0.05} & $(0.006,0.376)$ & $\begin{array}{l}\eta(0.011,0.070) \\
\text { PD: } 4.77, \text { Size: } 0.93 \\
\alpha: 0.865\end{array}$ & $\begin{array}{l}\eta(0.003,1.032) \\
\text { PD: } 6.63, \text { Size: } 1.04, \\
\alpha: 0.135\end{array}$ \\
\hline & $\begin{array}{r}0.5 \\
\end{array}$ & 0.5 & \\
\hline & $10 \sigma_{\mathrm{A}}=\sigma_{\mathrm{B}}$ & $10 \sigma_{\mathrm{A}}=\sigma_{\mathrm{B}}$ & $10 \sigma_{\mathrm{A}}=\sigma_{\mathrm{B}}$ \\
\hline \multirow[t]{3}{*}{0} & $(0.006,0.314)$ & $\begin{array}{l}\eta(0.011,0.045) \\
\text { PD: } 4.86, \text { Size: } 0.93 \\
\alpha: 0.837\end{array}$ & $\begin{array}{l}\eta(0.003,0.994) \\
\text { PD: } 7.58, \text { Size: } 1.02 \\
\alpha: 0.163\end{array}$ \\
\hline & 0.5 & & 1 \\
\hline & $10 \sigma_{\mathrm{A}}=\sigma_{\mathrm{B}}$ & $10 \sigma_{\mathrm{A}}=\sigma_{\mathrm{B}}$ & $10 \sigma_{\mathrm{A}}=\sigma_{\mathrm{B}}$ \\
\hline \multirow[t]{3}{*}{-0.05} & $(0.006,0.300)$ & $\begin{array}{l}\eta(0.011,0.033) \\
\text { PD: } 4.86, \text { Size: } 0.93 \\
\alpha: 0.835\end{array}$ & $\begin{array}{l}\eta(0.003,1.043) \\
\text { PD: } 7.78, \text { Size: } 1.01 \\
\alpha: 0.165\end{array}$ \\
\hline & 川 & 0.5 & $\pi$ \\
\hline & $10 \sigma_{\mathrm{A}}=\sigma_{\mathrm{B}}$ & $10 \sigma_{\mathrm{A}}=\sigma_{\mathrm{B}}$ & $10 \sigma_{\mathrm{A}}=\sigma_{\mathrm{B}}$ \\
\hline \multirow[t]{3}{*}{-0.1} & $(0.006,0.295)$ & $\begin{array}{l}\eta(0.011,0.025) \\
\text { PD: } 4.84, \text { Size: } 0.93 \\
\alpha: 0.839\end{array}$ & $\begin{array}{l}\eta(0.003,1.110) \\
\text { PD: } 7.86, \text { Size: } 1.01 \\
\alpha: 0.161\end{array}$ \\
\hline & 0.5 & 0.5 & $\pi$ \\
\hline & $10 \sigma_{\mathrm{A}}=\sigma_{\mathrm{B}}$ & $10 \sigma_{\mathrm{A}}=\sigma_{\mathrm{B}}$ & $10 \sigma_{\mathrm{A}}=\sigma_{\mathrm{B}}$ \\
\hline
\end{tabular}


Table 3.B.2: Critical points for the different binary mixtures depending on the non-additivity of component $B$, see also Figure $\mathbf{3 . 4 \mathbf { b }}$ and phase separated concentrations and volume fraction $\alpha$ of the different mixtures for specific parent concentration $\left(\eta_{A_{\text {parent }}}=0.010, \eta_{B_{\text {parent }}}=0.200\right)$, depending on the non-additivity of component $B, P D=12.00$.

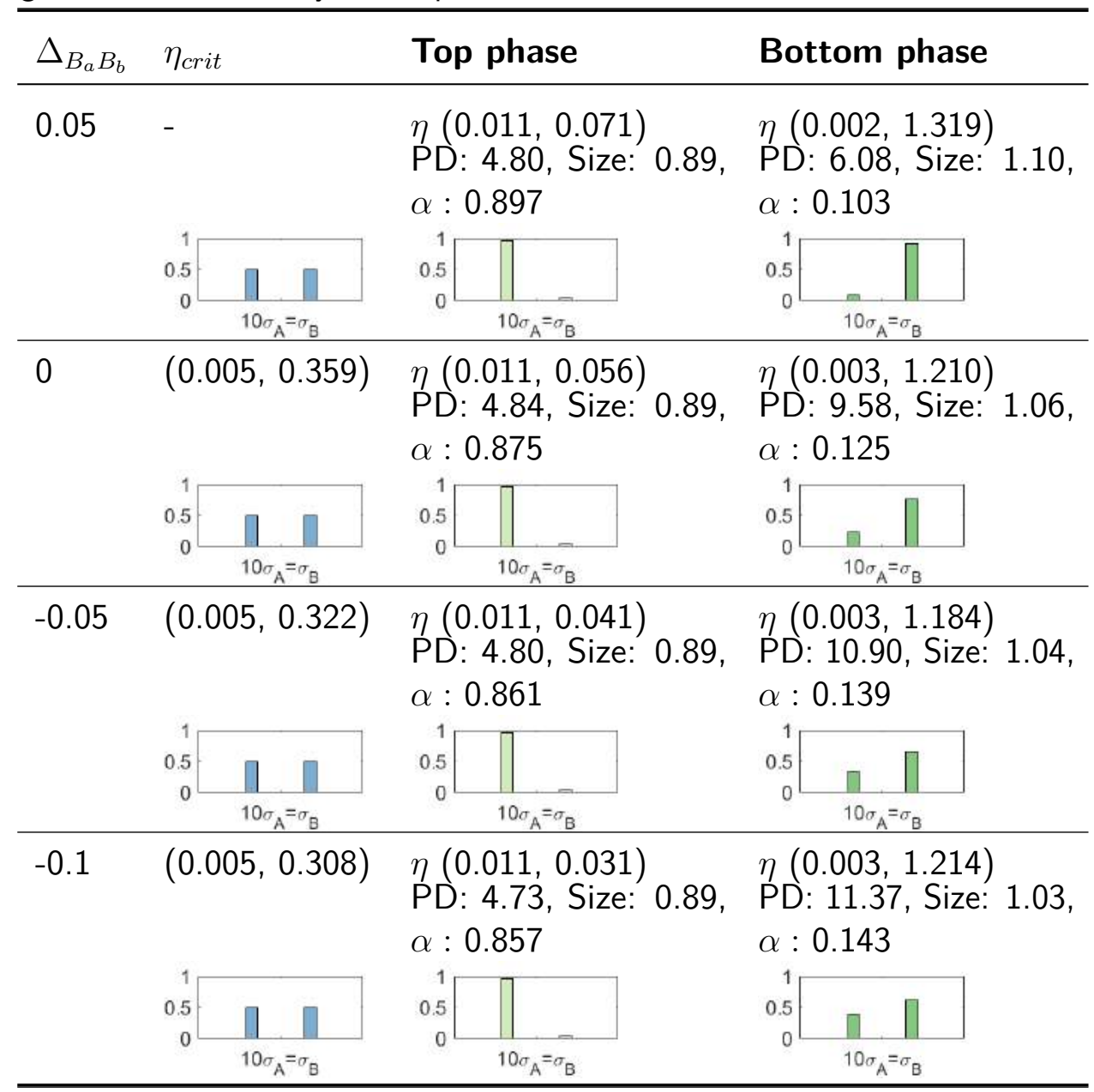


B. Fractionation

Table 3.B.3: Critical points for the different binary mixtures depending on the non-additivity of component $B$, see also Figure 3.6a and phase separated concentrations and volume fraction $\alpha$ of the different mixtures for specific parent concentration $\left(\eta_{A_{\text {parent }}}=0.010, \eta_{B_{\text {parent }}}=0.200\right)$, depending on the non-additivity of component $B, P D=6.93$.

\begin{tabular}{|c|c|c|c|}
\hline$\Delta_{B_{a} B_{b}}$ & $\eta_{\text {crit }}$ & Top phase & Bottom phase \\
\hline \multirow[t]{3}{*}{0.100} & $(0.006,0.374)$ & $\begin{array}{l}\eta(0.011,0.055) \\
\text { PD: } 7.41, \text { Size: } 0.92 \\
\alpha: 0.850\end{array}$ & $\begin{array}{l}\eta(0.003,1.024) \\
\text { PD: } 2.55, \text { Size: } 1.04 \\
\alpha: 0.159\end{array}$ \\
\hline & 0.5 & & $\begin{array}{r}1 \\
0.5\end{array}$ \\
\hline & $10 \sigma_{\mathrm{A}}=\sigma_{\mathrm{B}}$ & $10 \sigma_{\mathrm{A}}=\sigma_{\mathrm{B}}$ & $10 \sigma_{\mathrm{A}}=\sigma_{\mathrm{B}}$ \\
\hline \multirow[t]{3}{*}{0.05} & $(0.006,0.314)$ & $\begin{array}{l}\eta(0.011,0.048) \\
\text { PD: } 7.62, \text { Size: } 0.92 \\
\alpha: 0.837\end{array}$ & $\begin{array}{l}\eta(0.004,0.978) \\
\text { PD: } 4.28, \text { Size: } 1.03 \\
\alpha: 0.163\end{array}$ \\
\hline & $\begin{array}{r}0.5 \\
0\end{array}$ & 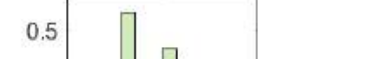 & 0.5 \\
\hline & $10 \sigma_{\mathrm{A}}=\sigma_{\mathrm{B}}$ & $10 \sigma_{\mathrm{A}}=\sigma_{\mathrm{B}}$ & $10 \sigma_{\mathrm{A}}=\sigma_{\mathrm{B}}$ \\
\hline \multirow[t]{3}{*}{0} & $(0.006,0.290)$ & $\begin{array}{l}\eta(0.011,0.037) \\
\text { PD: } 7.92, \text { Size: } 0.93 \\
\alpha: 0.822\end{array}$ & $\begin{array}{l}\eta(0.004,0.951) \\
\text { PD: } 5.57, \text { Size: } 1.02 \\
\alpha: 0.178\end{array}$ \\
\hline & \multirow{2}{*}{ 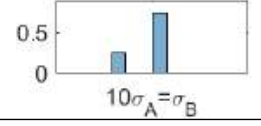 } & \multirow{2}{*}{${ }_{0}^{0.5} \prod \square$} & $\begin{array}{r}0.5 \\
0\end{array}$ \\
\hline & & & $10 \sigma_{\mathrm{A}}=\sigma_{\mathrm{B}}$ \\
\hline \multirow[t]{3}{*}{-0.05} & $(0.006,0.279)$ & $\begin{array}{l}\eta(0.011,0.028) \\
\text { PD: } 8.20, \text { Size: } 0.94 \\
\alpha: 0.816\end{array}$ & $\begin{array}{l}\eta(0.004,0.967) \\
\text { PD: } 6.21, \text { Size: } 1.01 \\
\alpha: 0.184\end{array}$ \\
\hline & \multirow{2}{*}{\begin{tabular}{r|r|}
0.5 & $\prod_{0} \prod_{10 \sigma_{\mathrm{A}}=\sigma_{\mathrm{B}}}$ \\
\end{tabular}} & \multirow{2}{*}{$r_{0}^{0.5} \prod_{10 \sigma_{\mathrm{A}}=\sigma_{\mathrm{B}}}$} & $\underset{0}{0.5} \square$ \\
\hline & & & $10 \sigma_{\mathrm{A}}=\sigma_{\mathrm{B}}$ \\
\hline \multirow[t]{3}{*}{-0.1} & $(0.006,0.277)$ & $\begin{array}{l}\eta(0.011,0.021) \\
\text { PD: } 8.35, \text { Size: } 0.95 \\
\alpha: 0.818\end{array}$ & $\begin{array}{l}\eta(0.003,1.002) \\
\text { PD: 6.53, Size: } 1.01 \\
\alpha: 0.182\end{array}$ \\
\hline & 0.5 & 0.5 & 0.5 \\
\hline & $10 \sigma_{\mathrm{A}}=\sigma_{\mathrm{B}}$ & $10 \sigma_{\mathrm{A}}=\sigma_{\mathrm{B}}$ & $10 \sigma_{\mathrm{A}}=\sigma_{\mathrm{B}}$ \\
\hline
\end{tabular}


Table 3.B.4: Critical points for the different binary mixtures depending on the non-additivity of component $B$, see also Figure 3.6b and phase separated concentrations and volume fraction $\alpha$ of the different mixtures for specific parent concentration $\left(\eta_{A_{\text {parent }}}=0.010, \eta_{B_{\text {parent }}}=0.200\right)$, depending on the non-additivity of component $B, P D=6.93$.

\begin{tabular}{|c|c|c|c|c|}
\hline$\Delta_{B_{a} B_{b}}$ & $\eta_{\text {crit }}$ & Top phase & Middle phase & Bottom phase \\
\hline \multirow[t]{3}{*}{0.100} & - & $\begin{array}{l}\eta(0.011,0.065) \\
\text { PD: } 3.05 \\
\text { Size: } 0.97 \\
\alpha: 0.819\end{array}$ & $\begin{array}{l}\eta(0.005,0.643) \\
\text { PD: } 4.43 \\
\text { Size: } 0.97 \\
\alpha: 0.097\end{array}$ & $\begin{array}{l}\eta(0.002,1.376) \\
\text { PD: } 2.88, \\
\text { Size: } 1.11, \\
\alpha: 0.04\end{array}$ \\
\hline & 0.5 & $\begin{array}{r}1 \\
0.5\end{array}$ & $\begin{array}{r}1 \\
0.5\end{array}$ & $\begin{array}{r}1 \\
0.5\end{array}$ \\
\hline & $10 \sigma_{\mathrm{A}}=\sigma_{\mathrm{B}}$ & $10 \sigma_{\mathrm{A}}=\sigma_{\mathrm{B}}$ & $10 \sigma_{\mathrm{A}}=\sigma_{\mathrm{B}}$ & $10 \sigma_{\mathrm{A}}=\sigma_{\mathrm{B}}$ \\
\hline \multirow[t]{3}{*}{0.05} & $(0.006,0.325)$ & $\begin{array}{l}\eta(0.011,0.050) \\
\text { PD: } 2.96, \\
\text { Size: } 0.97 \\
\alpha: 0.813\end{array}$ & & $\begin{array}{l}\eta(0.004,0.854) \\
\text { PD: } 7.35, \\
\text { Size: } 1.01, \\
\alpha: 0.187\end{array}$ \\
\hline & $\begin{array}{r}1 \\
0.5\end{array}$ & $\begin{array}{r}1 \\
0.5 \\
0\end{array} \square$ & & \begin{tabular}{r|l|l|}
1 & \\
0.5 & $\square$ \\
0 & $\square$
\end{tabular} \\
\hline & $10 \sigma_{\mathrm{A}}=\sigma_{\mathrm{B}}$ & $10 \sigma_{\mathrm{A}}=\sigma_{\mathrm{B}}$ & & $10 \sigma_{\mathrm{A}}=\sigma_{\mathrm{B}}$ \\
\hline \multirow[t]{3}{*}{0} & $(0.006,0.326)$ & $\begin{array}{l}\eta(0.011,0.044) \\
\text { PD: } 2.36, \\
\text { Size: } 0.96, \\
\alpha: 0.826\end{array}$ & & $\begin{array}{l}\eta(0.004,0.941) \\
\text { PD: } 7.33, \\
\text { Size: } 1.01, \\
\alpha: 0.174\end{array}$ \\
\hline & 0.5 & $\begin{array}{r}1 \\
0.5 \\
0\end{array}$ & & $\begin{array}{rll}0.5 & \square \square\end{array}$ \\
\hline & $10 \sigma_{\mathrm{A}}=\sigma_{\mathrm{B}}$ & $10 \sigma_{\mathrm{A}}=\sigma_{\mathrm{B}}$ & & $10 \sigma_{\mathbf{A}}=\sigma_{\mathbf{B}}$ \\
\hline \multirow[t]{3}{*}{-0.05} & $(0.006,0.331)$ & $\begin{array}{l}\eta(0.011,0.040) \\
\text { PD: } 1.84, \\
\text { Size: } 0.96, \\
\alpha: 0.837\end{array}$ & & $\begin{array}{l}\eta(0.003,1.025) \\
\text { PD: } 7.31, \\
\text { Size: } 1.01, \\
\alpha: 0.163\end{array}$ \\
\hline & 0.5 & $\begin{array}{r}1 \\
0.5\end{array}$ & & $\begin{array}{r}1 \\
0.5\end{array}$ \\
\hline & $10 \sigma_{\mathrm{A}}=\sigma_{\mathrm{B}}$ & $10 \sigma_{\mathrm{A}}=\sigma_{\mathrm{B}}$ & & $10 \sigma_{\mathrm{A}}=\sigma_{\mathrm{B}}$ \\
\hline \multirow[t]{3}{*}{-0.1} & $(0.005,0.337)$ & $\begin{array}{l}\eta(0.011,0.037) \\
\text { PD: } 1.42, \\
\text { Size: } 0.96, \\
\alpha: 0.848\end{array}$ & & $\begin{array}{l}\eta(0.003,1.106 \\
\text { PD: } 7.29 \\
\text { Size: } 1.01 \\
\alpha: 0.142\end{array}$ \\
\hline & 0.5 . & 0.5 & & 0.5 \\
\hline & $10 \sigma_{\mathrm{A}}=\sigma_{\mathrm{B}}$ & $10 \sigma_{\mathrm{A}}=\sigma_{\mathrm{B}}$ & & $10 \sigma_{\mathrm{A}}=\sigma_{\mathrm{B}}$ \\
\hline
\end{tabular}


B. Fractionation

Table 3.B.5: Critical points for the different binary mixtures depending on the non-additivity of component $B$, see also Figure 3.5a and phase separated concentrations and volume fraction $\alpha$ of the different mixtures for specific parent concentration $\left(\eta_{A_{\text {parent }}}=0.010, \eta_{B_{\text {parent }}}=0.200\right)$, depending on the non-additivity of component $B, P D=4.80$.

\begin{tabular}{|c|c|c|c|}
\hline$\Delta_{B_{a} B_{b}}$ & $\eta_{\text {crit }}$ & Top phase & Bottom phase \\
\hline \multirow[t]{3}{*}{0.100} & $(0.007,0.295)$ & $\begin{array}{l}\eta(0.011,0.039) \\
\text { PD: } 8.51, \text { Size: } 0.94 \\
\alpha: 0.817\end{array}$ & $\begin{array}{l}\eta(0.004,0.918) \\
\text { PD: } 1.77, \text { Size: } 1.01 \\
\alpha: 0.183\end{array}$ \\
\hline & $\begin{array}{r}1 \\
0.5\end{array}$ & $\begin{array}{r}1 \\
0.5\end{array}$ & $\begin{array}{r}1 \\
0.5\end{array}$ \\
\hline & $0 \quad 10 \sigma_{\mathrm{A}}=\sigma_{\mathrm{B}}$ & $10 \sigma_{\mathrm{A}}=\sigma_{\mathrm{B}}$ & $10 \sigma_{\mathrm{A}}=\sigma_{\mathrm{B}}$ \\
\hline \multirow[t]{3}{*}{0.05} & $(0.007,0.284)$ & $\begin{array}{l}\eta(0.011,0.036) \\
\text { PD: 8.40, Size: } 0.95 \\
\alpha: 0.811\end{array}$ & $\begin{array}{l}\eta(0.004,0.904) \\
\text { PD: } 2.69, \text { Size: } 1.01 \\
\alpha: 0.189\end{array}$ \\
\hline & $\begin{array}{r}1 \\
0.5\end{array}$ & $\begin{array}{lll}0.5 & \square\end{array}$ & $\begin{array}{r}1 \\
0.5\end{array}$ \\
\hline & $10 \sigma_{\mathrm{A}}=\sigma_{\mathrm{B}}$ & $10 \sigma_{\mathrm{A}}=\sigma_{\mathrm{B}}$ & $10 \sigma_{\mathrm{A}}=\sigma_{\mathrm{B}}$ \\
\hline \multirow[t]{3}{*}{0} & $(0.007,0.276)$ & $\begin{array}{l}\eta(0.011,0.032) \\
\text { PD: } 8.12, \text { Size: } 0.96 \\
\alpha: 0.805\end{array}$ & $\begin{array}{l}\eta(0.004,0.895) \\
\text { PD: } 3.53, \text { Size: } 1.01 \\
\alpha: 0.195\end{array}$ \\
\hline & 0.5 & 0.5 & 0.5 \\
\hline & $10 \sigma_{\mathrm{A}}=\sigma_{\mathrm{B}}$ & $10 \sigma_{\mathrm{A}}=\sigma_{\mathrm{B}}$ & $10 \sigma_{\mathrm{A}}=\sigma_{\mathrm{B}}$ \\
\hline \multirow[t]{3}{*}{-0.05} & $(0.007,0.271)$ & $\begin{array}{l}\eta(0.012,0.028) \\
\text { PD: } 7.58, \text { Size: } 0.97 \\
\alpha: 0.803\end{array}$ & $\begin{array}{l}\eta(0.004,0.899) \\
\text { PD: } 4.11, \text { Size: } 1.00 \\
\alpha: 0.197\end{array}$ \\
\hline & 0.5 & $\pi$ & 0.5 \\
\hline & $10 \sigma_{\mathrm{A}}=\sigma_{\mathrm{B}}$ & $10 \sigma_{\mathrm{A}}=\sigma_{\mathrm{B}}$ & $10 \sigma_{\mathrm{A}}=\sigma_{\mathrm{B}}$ \\
\hline \multirow[t]{3}{*}{-0.1} & $(0.007,0.270)$ & $\begin{array}{l}\eta(0.012,0.025) \\
\text { PD: } 6.79, \text { Size: } 0.98 \\
\alpha: 0.803\end{array}$ & $\begin{array}{l}\eta(0.004,0.914) \\
\text { PD: } 4.46, \text { Size: } 1.00 \\
\alpha: 0.197\end{array}$ \\
\hline & 0.5 & $0.5 \square$ & 0.5 \\
\hline & $10 \sigma_{\mathrm{A}}=\sigma_{\mathrm{B}}$ & $10 \sigma_{\mathrm{A}}=\sigma_{\mathrm{B}}$ & $10 \sigma_{\mathrm{A}}=\sigma_{\mathrm{B}}$ \\
\hline
\end{tabular}


Table 3.B.6: Critical points for the different binary mixtures depending on the non-additivity of component $B$, see also Figure 3.5b and phase separated concentrations and volume fraction $\alpha$ of the different mixtures for specific parent concentration $\left(\eta_{A_{\text {parent }}}=0.010, \eta_{B_{\text {parent }}}=0.200\right)$, depending on the non-additivity of component $B, P D=4.80$.

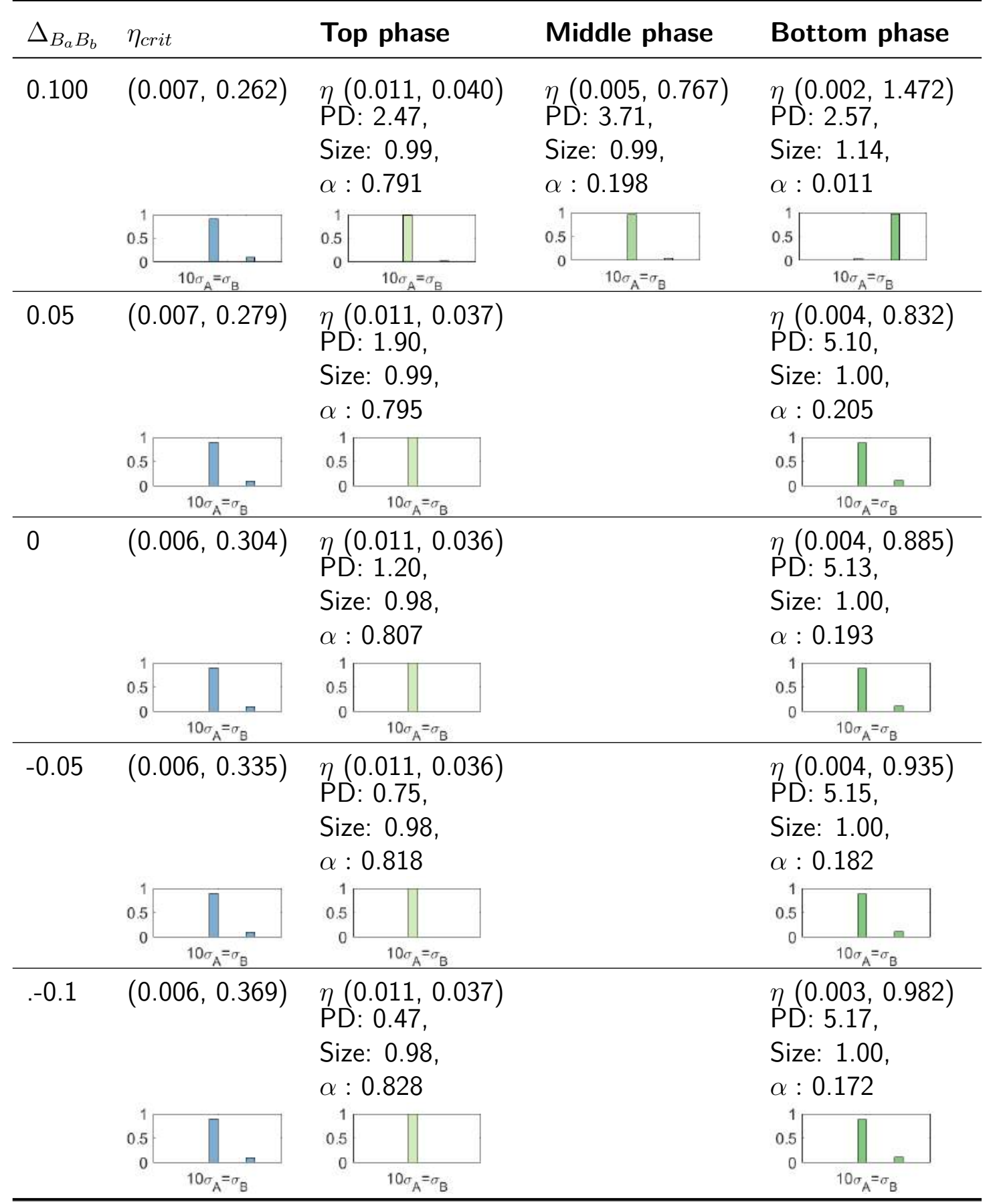




\section{4}

\section{Effect of third component on the phase behavior of a binary mixture of hard spheres in solution}




\begin{abstract}
We study the liquid phase behavior of ternary mixtures of monodisperse hard spheres in solution. The interactions are modeled in terms of the second virial coefficient and can be additive hard sphere (HS) or non-additive hard sphere (NAHS) interactions. We give the set of equations that defines the phase diagram for mixtures of three components. We calculate the theoretical liquid-liquid phase separation boundary for two phase separation (the binodal) and if applicable the three phase boundary, as well as the plait points and the spinodal. The sizes of the three components are fixed. The first component $(A)$ is the smallest one, the second component $(B)$ is four times the size of the smallest component, and the third $(C)$ component is three times the size of the smallest one. The interaction between the first two components are fixed, this $A B$ sub-mixture shows phase separation. The interactions for the component $C$ with the other two components is varied. The component $C$ can be compatible or incompatible with components $A$ and $B$. Depending on the compatibility of the components the phase diagram is altered. The addition of the third component has an influence on the phase boundary, plait points, stability region, fractionation and volume ratio between the different phases. When all sub-mixtures $(A B, A C$, and $B C)$ show phase separation, a three-phase system becomes possible when the incompatibility among all components is high enough. The position and size of the three-phase region is dependent on the interactions between the different sub-mixtures. we study the fractionation off all components depending on specific parent concentrations.
\end{abstract}




\subsection{Introduction}

A lot of research, both theoretical and experimental, has been focused on the phase behavior of two components in solution. However, most products consist out of more components. The investigation of multicomponent phase behavior has a considerable amount of challenges. From an experimental point of view, the number of samples to prepare to get an insight into the phase behavior at different concentrations in the phase diagram increases significantly with the number of considered components. From a theoretical point of view, the number of pairwise interactions for a mixtures with $n$ components taken into account increases according to $\frac{n(n+1)}{2}$. The number of interactions increases even more when higher order interactions are taken into account. Depending on the interactions between all the components, the theoretical number of possible phases that can be formed will increase as well, as follows from the Gibbs phase rule. The Gibbs phase rule gives the relationship between the number of possible phases and the degrees of freedom ( $d o f)$ for a system: $d o f=n+2-f$, in which $n$ is the number of components and $f$ is the maximum number of phases in equilibrium. The degrees of freedom for the system are for example the mole fraction of the components, the temperature or the pressure.

When looking at multicomponent systems, one may distinguish chemically different components and chemically equivalent components that only differ in size. The latter is often met in experimental conditions since many components show polydispersity in their size, e.g. mixtures containing polymers such as poly-ethylene glycol, dextran, gelatin,...(Edelman et al., 2003, 2001; Zhao et al., 2016; Chu et al., 1996). In this work we will focus on mixtures containing three distinct types of hard spheres in solution with non-additive pairwise interactions. They can be seen as a model for a system containing different components, e.g. colloids in solution.

Depletion interactions in systems with more than two components become more complicated. This has been shown by Ji and Walz (2013), who investigated the depletion interaction in a highly asymmetric ternary system. They found out that the energy profile between two particles is significantly altered due to the addition of bi-disperse depletants: the pair-potential has an additional attractive energy well and an extended repulsive barrier that are of significant magnitude to alter the stability of the dispersion of the larger particles. Also Park and Conrad (2017) studied the depletion interaction in a system of colloids and bi-disperse polymers. They report that the bi-disperse polymers could not be treated as homogeneous depletants. Both sizes of the polymers contributed to the effective interactions.

Theoretical work on the phase behavior and stability of solutions with more than two components has also been important in understanding cellular systems. Cells contain a large number of components in solution and it has been hypothesized that demixing and phase 
separation leads to the organization within cells (Harmon et al., 2017). Sear and Cuesta (2003) investigated the instability in these kind of mixtures with many components. They approximated the second virial coefficients of the pairwise interactions of the components in the system using random matrix theory. A positive virial coefficient indicates a repulsive interaction between the two components. A negative virial coefficient on the other hand indicates an attractive interaction between the two components, leading to aggregation and complex formation. Since the virial coefficient matrix is filled in randomly from a distribution it is possible that the matrix contains negative values. An unstable mixture will therefore not necessarily stem from depletion interaction, but might be caused by the formation of complexes and aggregates.

Some experimental work on mixtures with more than two components that demix into multiple phase systems has focused on methods to aid in the partitioning of proteins or cell contents. Each of the phases in these systems has a different density and relatively low surface tension aiding in the separation. An example of such a system is the system containing the high-molecular colloids dextran, ficoll and poly-ethylene-glycol (Hartman et al., 1974; Albertsson and Birkenmeier, 1988). Similar results were obtained by Ruan et al. (2006) for the system of the colloids dextran and polyethylene-glycol with the surfactant Triton X-100. Mixtures with an even higher number of components (both colloids and surfactants) and a corresponding higher number of phases in equilibrium were studied by Mace et al. (2012). They report systems of up to six phases, each enriched in one of the polymers and depleted in the others.

A more in depth experimental study on the phase behavior and phase diagram of a mixture of three components and the three-phase dynamics was done by Beck-Candanedo et al. (2007). They studied the phase behavior of rods in the presence of two types of dextran. At high enough concentrations, rod-like particles such as cellulose nano-crystals form a two phase system of an isotropic and an nematic phase. The addition of the different types of dextran leads to a widening of the two phase coexistence region or the formation of an additional isotropic phase, resulting in a three-phase system.

In our current study we aim to get a better understanding of how the addition of a third component influences the phase behavior of two components that show phase separation. We will study the position of the phase separation boundary, the spinodal the plait points, and the fractionation. We model the interactions between the components using the first and second order virial coefficient (Section 4.2.1). In Section 4.2.2 we describe the equations for the stability boundary, the spinodal, in Section 4.2.3 we describe the equations for the plait point line and finally in Section 4.2.4 we describe the equations defining the phase boundary. With the expressions in Section 4.2 we have enough to calculate the full phase diagram for a variety of mixtures described in Section 4.3. We add a third 
component $C$ to a binary mixture $A B$ that phase separates. First the component $C$ is compatible with both $A$ and $B$ (Section 4.3.1). In the next section (Section 4.3.2), $C$ is incompatible with $A$ or $B$, and compatible with $B$ or $A$ respectively. In the third section (Section 4.3.3) $C$ is incompatible with both $A$ and $B$. Subsequently we investigate the concentration of each component in the phase separated mixtures at specific parent concentrations (Section 4.3.4). In the last section we compare three component systems with a different particle size and different interactions that show three phase separation (Section 4.3.5).

\subsection{Theory}

We show the equations used for the calculations of the ternary phase diagram of the different studied systems: the set of equations defining the stability boundary, the plait points, and phase boundaries of a mixture. All sets of equations are solved in Matlab R2017b. For a more detailed derivation of the equations, we refer to Sturtewagen and van der Linden (2019) and references therein. The set of equations determining the phase diagram originate from expressions for the osmotic pressure and chemical potentials which should be equal among the coexisting phases in equilibrium.

\subsubsection{Osmotic virial coefficient}

The osmotic pressure, $\Pi$, of a solution at a temperature $T$, can be written as a virial expansion, similar to the virial expansion of the universal gas law for real gases (Hill, 1986):

$$
\beta \Pi=\rho+B_{2}\left(T, \mu_{s}\right) \rho^{2}+B_{3}\left(T, \mu_{s}\right) \rho^{3}+\ldots
$$

with $\beta=\frac{1}{k T}, k$ the Boltzmann's constant, $\rho$ the number density of the component $\left(\frac{N_{\nu}}{V}\right), B_{2}$ the second virial coefficient, and $B_{3}$ the third virial coefficient. The second virial coefficient accounts for the increase in osmotic pressure due to particle pairwise interaction. The third virial coefficient accounts for the interaction between three particles in a variety of configurations. The equation can be expanded for higher densities with $B_{n}$, the $n^{\text {th }}$ virial coefficient, which accounts for the interaction between $n$ different particles.

In this work we will limit the virial expansion to the second virial coefficient, which is given by (Lekkerkerker and Tuinier, 2011):

$$
B\left(T, \mu_{s}\right)=2 \pi \int_{0}^{\infty} r^{2}\left(1-\exp \left[-\beta W\left(r, \mu_{s}\right)\right]\right) d r
$$


in which $\mu_{s}$ is the chemical potential of the solution, $W(r)$ is the interaction potential between the particles, and $r$ is the distance between them.

For additive hard sphere (HS) interaction, the interaction potential for two particles (of the same species or different species) is given by:

$$
W(r)_{H S}= \begin{cases}0, & r>\sigma_{i j} \\ \infty, & r \leq \sigma_{i j}\end{cases}
$$

with $\sigma_{i j}=\left(\sigma_{i}+\sigma_{j}\right) / 2$, the distance between the centers of the two particles, and $\sigma_{i}$ and $\sigma_{j}$ their respective diameter.

For non-additive hard spheres (NAHS), the distance of the closest approach of the centers of the two particles of different species can be closer or further than the distance between their centers (Roth et al., 2001). The closest distance then becomes: $\sigma_{i j}=\left(\left(\sigma_{i}+\sigma_{j}\right) / 2\right)(1+\Delta)$, in which $\Delta(\geq-1)$ accounts for the non-additivity of the interaction between the particles. When $\Delta>0$ the distance of closest approach of both spheres increases and when $\Delta<0$ the distance of closest approach decreases compared to that due to HS interaction only. For additive hard sphere interaction $\Delta=0$.

In a mixture with $n$ distinguishable components in a solution, there are two main types of two particle interactions that can occur: between particles of the same species and particles of different species. For the system we are studying, $n=3$ (Figure 4.1).

For the second virial coefficient given by Eq. (4.2), using the interaction potential defined in Eq. (4.3), we find:

$$
\begin{aligned}
& B_{x x}=\frac{2 \pi}{3}\left(\sigma_{x}\right)^{3} \\
& B_{x y}=\frac{2 \pi}{3}\left(\left(\frac{\sigma_{x}+\sigma_{y}}{2}\right)(1+\Delta)\right)^{3}
\end{aligned}
$$

where $B_{x x}$ is the second virial coefficient for particles of the same species (assumed to be HS) and $B_{x y}$ is the second virial coefficient of particles of different species, which can be HS or NAHS.

The general equation for the osmotic pressure for a dilute mixture of 3 components, limited to the second virial coefficient, is given by (Sturtewagen and van der Linden, 2019):

$$
\begin{aligned}
\beta \Pi= & \rho_{A}+\rho_{B}+\rho_{C}+B_{A A} \rho_{A}^{2}+2 B_{A B} \rho_{A} \rho_{B}+2 B_{A C} \rho_{A} \rho_{C}+B_{B B} \rho_{B}^{2} \\
& +2 B_{B C} \rho_{B} \rho_{C}+B_{C C} \rho_{C}^{3} \\
= & \rho_{A}+\rho_{B}+\rho_{C}+\sum_{i}^{3} \sum_{j}^{3} B_{i j} \rho_{i} \rho_{j}
\end{aligned}
$$




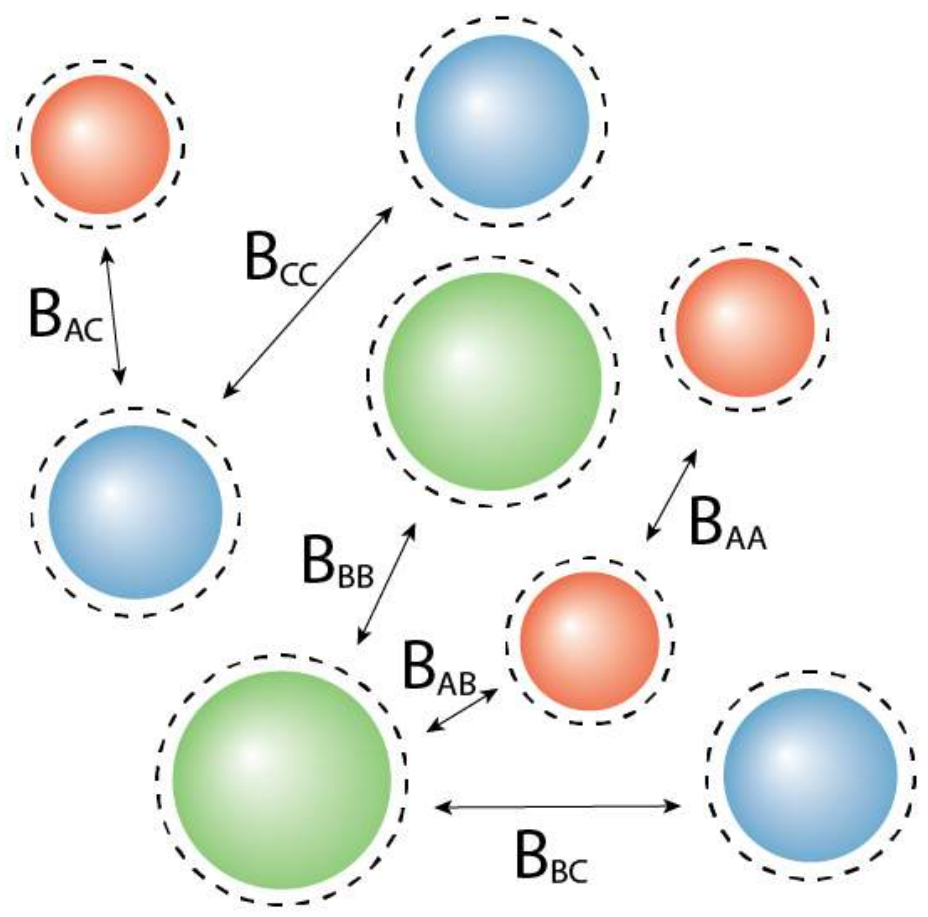

Figure 4.1: Graphical representation of a simple ternary mixture $A B C$, second virial coefficients are indicated.

\subsubsection{Stability of a mixture}

The stability of a mixture is dependent on the second derivative of the free energy. If the second derivative of the mixture becomes zero, the mixture is at the boundary of becoming unstable. Unstable mixtures have a negative second derivative (Heidemann, 1975; Beegle et al., 1974).

The differential of the free energy of a mixture is given by (Hill, 1986):

$$
d A=-S d T-p d V+\sum_{i}^{n} \mu_{i} d N_{i}
$$

in which $\mu_{i}$, the chemical potential (the first partial derivative of the free energy with respect to number of particles $\left.\left(N_{i}\right)\right)$ for component $i$ is given by:

$$
\mu_{i}=\mu_{i}^{0}+k T \ln \left(\rho_{i}\right)+2 k T\left(\sum_{j}^{n} B_{i j} \rho_{j}\right)
$$

For a mixture with 3 distinguishable components, the second partial derivatives can be represented by a $3 \times 3$ matrix of the first partial derivatives of the chemical potential of each component.

This results in the following stability matrix: 


$$
\begin{aligned}
M_{1} & =\left[\begin{array}{lll}
\frac{\partial \mu_{A}}{\partial N_{A}} & \frac{\partial \mu_{A}}{\partial N_{B}} & \frac{\partial \mu_{A}}{\partial N_{B}} \\
\frac{\partial \mu_{B}}{\partial N_{A}} & \frac{\partial \mu_{B}}{\partial N_{B}} & \frac{\partial \mu_{B}}{\partial N_{C}} \\
\frac{\partial \mu_{C}}{\partial N_{A}} & \frac{\partial \mu_{C}}{\partial N_{B}} & \frac{\partial \mu_{C}}{\partial N_{C}}
\end{array}\right] \\
= & {\left[\begin{array}{ccc}
2 B_{A A}+\frac{1}{\rho_{A}} & 2 B_{A B} & 2 B_{A C} \\
2 B_{A B} & 2 B_{B B}+\frac{1}{\rho_{B}} & 2 B_{B C} \\
2 B_{A C} & 2 B_{B C} & 2 B_{C C}+\frac{1}{\rho_{C}}
\end{array}\right] }
\end{aligned}
$$

The mixture is stable when all eigenvalues are positive (Solokhin et al., 2002). When on the other hand one of the eigenvalues is not positive, the mixture becomes unstable. The limit of stability is reached when the matrix has one zero eigenvalue and is otherwise positive definite, and is referred to as the spinodal (Heidemann and Khalil, 1980).

\subsubsection{Plait points}

In a binary mixture, the critical point is a stable point which lies on the stability limit (spinodal) (Heidemann and Khalil, 1980) and where the phase boundary and spinodal coincide. In mixtures of more components these critical points become plait points. Critical points and plait points are in general concentrations at which two or more phases are in equilibrium and become indistinguishable (Heidemann, 1994).

There are two criteria that can be used to find plait points. The first one is $\operatorname{det}\left(M_{1}\right)=0$, which is the equation for the spinodal. The other criterion is based on the fact that at the critical point, the third derivative of the free energy should also be zero. For a multicomponent system, this criterion can be reformulated using Legendre transforms as $\operatorname{det}\left(M_{2}\right)=0$ (Beegle et al., 1974; Reid and Beegle, 1977), where:

$$
M_{2}=\left[\begin{array}{lll}
\frac{\partial \mu_{A}}{\partial N_{A}} & \frac{\partial \mu_{A}}{\partial N_{B}} & \frac{\partial \mu_{A}}{\partial N_{C}} \\
\frac{\partial \mu_{B}}{\partial N_{A}} & \frac{\partial \mu_{B}}{\partial N_{B}} & \frac{\partial \mu_{B}}{\partial N_{C}} \\
\frac{\partial M_{1}}{\partial N_{A}} & \frac{\partial M_{1}}{\partial N_{B}} & \frac{\partial M_{1}}{\partial N_{C}}
\end{array}\right]
$$

Matrix $M_{2}$ is matrix $M_{1}$ with one of the rows replaced by the partial derivatives of the determinant of matrix $M_{1}$. Note: it does not matter which row of the matrix is replaced. 


$$
M_{2}=\left[\begin{array}{ccc}
2 B_{A A}+\frac{1}{\rho_{A}} & 2 B_{A B} & 2 B_{A C} \\
2 B_{A B} & 2 B_{B B}+\frac{1}{\rho_{B}} & 2 B_{B C} \\
P_{1} & P_{2} & P_{3}
\end{array}\right]
$$

with

$$
\begin{gathered}
P_{1}=-\frac{\left(\begin{array}{c}
-4 B_{B C}^{2} \rho_{B} \rho_{C}+2 B_{B B} \rho_{B}+2 B_{C C} \rho_{C} \\
+4 B_{B B} B_{C C} \rho_{B} \rho_{C}+1
\end{array}\right)}{\rho_{A}^{2} \rho_{B} \rho_{C}} \\
P_{2}=-\frac{\left(\begin{array}{c}
-4 B_{A C}^{2} \rho_{A} \rho_{C}+2 B_{A A} \rho_{A}+2 B_{C C} \rho_{C} \\
+4 B_{A A} B_{C C} \rho_{A} \rho_{C}+1
\end{array}\right)}{\left.\rho_{A} \rho_{B}^{2} \rho_{C}\right)} \\
P_{3}=-\frac{\left(\begin{array}{c}
-4 B_{A B}^{2} \rho_{A} \rho_{B}+2 B_{A A} \rho_{A}+2 B_{B B} \rho_{B} \\
+4 B_{A A} B_{B B} \rho_{A} \rho_{B}+1
\end{array}\right)}{\rho_{A} \rho_{B} \rho_{C}^{2}}
\end{gathered}
$$

In general, $P_{i}$ can be found using the following equation (Sturtewagen and van der Linden, 2019):

$$
P_{i}=-\frac{1}{\rho_{i}^{2}} M_{1,(i i)}
$$

in which, $M_{1,(i i)}$ is the minor of matrix $M_{1}$ at the $i^{\text {th }}$-row and $i^{\text {th }}$-column.

We note that for some of the mixtures, the set of equations $\left(\operatorname{det}\left(M_{1}\right)=0\right.$ and $\left.\operatorname{det}\left(M_{2}\right)=0\right)$, especially when all three components are incompatible, has solutions that are not on a phase boundary. We consider this a mathematical solution to the set of equations, but not a physical solution for a plait point, since plait points are defined to be concentrations on the phase boundary where two or more phases become indistinguishable. Therefore these solutions will be ignored when depicting the phase diagram.

\subsubsection{Phase boundary}

When a mixture becomes unstable and demixes into two or more phases, the chemical potential of each component and the osmotic pressure is the same in all phases (Hill, 1986), yielding. 


$$
\left\{\begin{array}{c}
\beta \Pi^{I}=\beta \Pi^{I I}=\cdots \\
\beta \mu_{1}^{I}=\beta \mu_{1}^{I I}=\cdots \\
\vdots \\
\beta \mu_{n}^{I}=\beta \mu_{n}^{I I}=\cdots
\end{array}\right.
$$

where the phases are denoted by $I, I I, \ldots$.

For a mixture containing 3 distinguishable components, that demixes into two phases, this results in 4 equations and $2 \times 3$ unknowns. If the mixture demixes into three phases, this results in $2 \times 3+2$ equations and $3 \times 3$ unknowns. To solve the set of equations without having to fix the concentration of one component and the ratio between the other components for at least one of the phases, we need extra equations. For the extra set of equations, we build on the fact that no particles are lost and no new particles are created during phase separation, and the fact that we assume that the total volume does not change.

For a system that separates into three phases we then obtain:

$$
\rho=\sum_{i}^{3} \rho_{i}=\frac{\sum_{i}^{3} N_{i}}{V}=\frac{\sum_{i}^{3} N_{i}^{I}+\sum_{i}^{3} N_{i}^{I I}+\sum_{i}^{3} N_{i}^{I I I}}{V^{I}+V^{I I}+V^{I I I}}
$$

which can be rewritten as (Sturtewagen and van der Linden, 2019):

$$
\begin{aligned}
\rho & =\alpha^{I} \sum_{i}^{3} \rho_{i}^{I}+\alpha^{I I} \sum_{i}^{3} \rho_{i}^{I I}+\left(1-\alpha^{I}-\alpha^{I I}\right) \sum_{i}^{3} \rho_{i}^{I I I} \\
& =\sum_{i}^{3} \rho_{i}
\end{aligned}
$$

with

$$
\alpha^{I}=\frac{V^{I}}{\sum_{i}^{f} V^{i}}
$$

in which $f$ denotes the number of phases.

This results in the following set of equations: 


$$
\left\{\begin{aligned}
\beta \Pi^{I} & =\beta \Pi^{I I}=\cdots \\
\beta \mu_{A}^{I} & =\beta \mu_{A}^{I I}=\cdots \\
\beta \mu_{B}^{I} & =\beta \mu_{B}^{I I}=\cdots \\
\beta \mu_{C}^{I} & =\beta \mu_{C}^{I I}=\cdots \\
\rho_{1} & =\alpha^{I} \rho_{A}^{I}+\cdots+\left(1-\sum_{i}^{f-1} \alpha^{i}\right) \rho_{A}^{f} \\
\rho_{2} & =\alpha^{I} \rho_{B}^{I}+\cdots+\left(1-\sum_{i}^{f-1} \alpha^{i}\right) \rho_{B}^{f} \\
\rho_{3} & =\alpha^{I} \rho_{C}^{I}+\cdots+\left(1-\sum_{i}^{f-1} \alpha^{i}\right) \rho_{C}^{f}
\end{aligned}\right.
$$

With Eq. (4.14), we have $2 \times 3+1$ unknowns and $2 \times 3+1$ equations for mixtures that separate into two phases. For mixtures that demix into three phases, we will have $3 \times 3+2$ unknowns and $3 \times 3+2$ equations. Therefore, the set of equations given by Eq. (4.14) allows for calculating the concentration of each component in each of the phases for any given parent concentration.

\subsection{Results and discussion}

We calculate the phase diagram for a variety of ternary mixture of hard spheres $(A, B$, and $C)$ with non-additive interactions. For all particles, the concentrations are expressed as a dimensionless parameter according to $\eta=\frac{\pi \rho \sigma^{3}}{6}$. We define a specific binary mixture of components $A$ and $B$, to which we add a third component $C$. The size ratio between the small sphere $A$ and the larger sphere $B$ is $q_{A B}=\sigma_{A} / \sigma_{B}=1 / 4$ and the non-additivity parameter $\Delta_{A B}=0.1$. The critical point for the binary mixture $A B$ is at $\eta_{\text {crit }}=(0.037,0.229)$. To this mixture we add a component $C$, with size ratio $q_{A C}=\sigma_{A} / \sigma_{C}=1 / 3$. The size of $C$ is therefore in between the sizes of $A$ and $B$. We vary the non-additivity parameters $\left(\Delta_{A C}\right.$ and $\Delta_{B C}$ ) between components $A$ and $C$, and $B$ and $C$ respectively, from $\Delta=-0.1$ to $\Delta=0.1$, simulating a range of thermodynamic compatibility and incompatibility between the three components. The phase diagram of this binary mixture of $A$ and $B$ can be found in Figure 4.2. We calculate the plait points, the phase separation boundary, and the spinodal of the various ternary mixtures (see Figures 4.3 to 4.11 ).

Mixtures are able to demix when the stability matrix (Eq. (4.9)) has a negative eigenvalue at low enough concentrations (in our case we look at concentrations $\eta<0.5$ ). An easy way to check for the possibility of phase separation for binary mixtures is using the following 


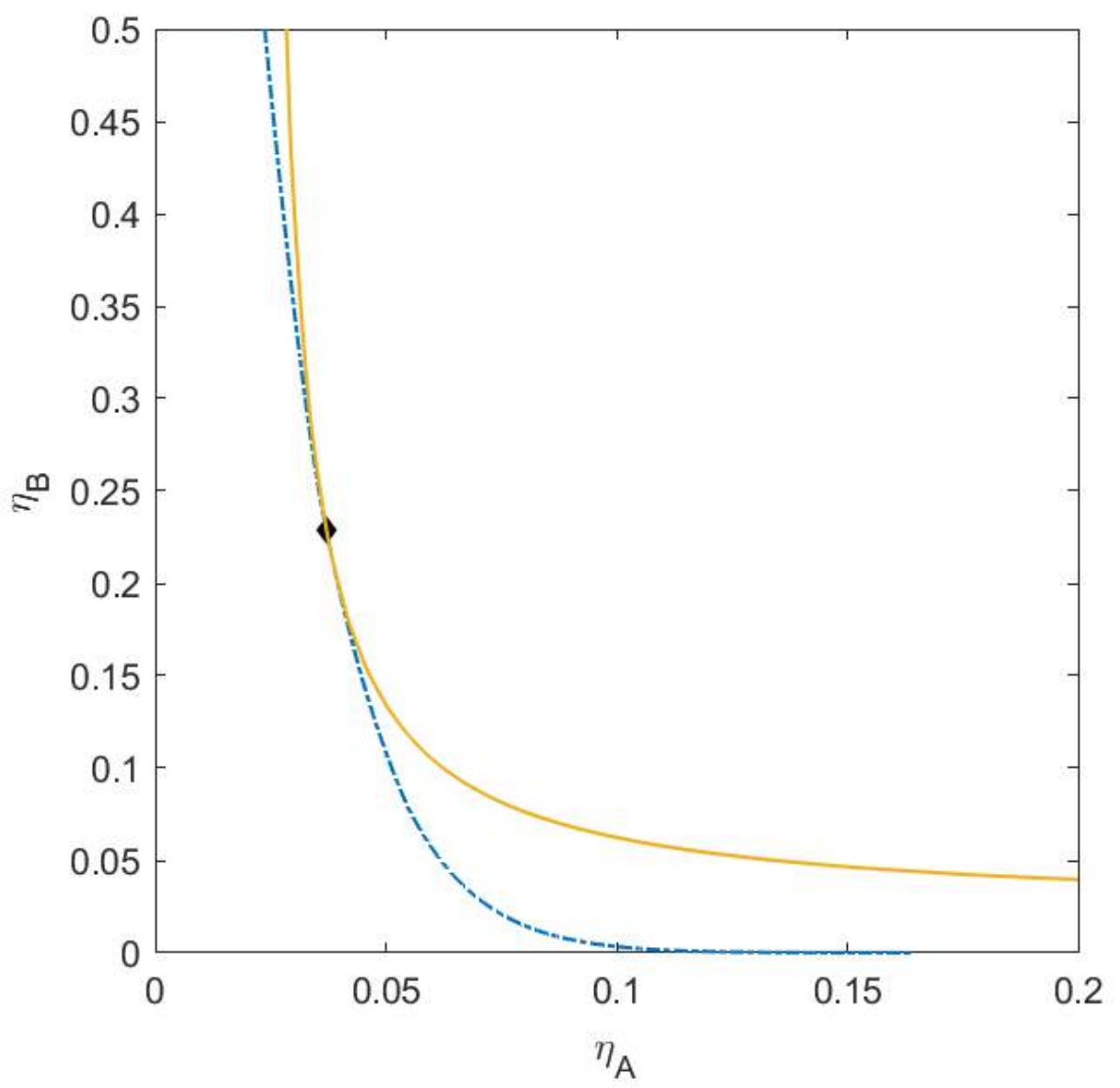

Figure 4.2: Phase diagram for monodisperse binary (component $A$ and $B$ ) non-additive hard sphere mixture with size ratio $q=\sigma_{A} / \sigma_{B}=1 / 4$ and $\Delta_{A B}=0.1$, plotted as a function of the partial packing fractions, $\eta_{A}$ and $\eta_{B}$. The spinodal (solid yellow line) and binodal (dashed blue line) meet each other at the critical point (black diamond).

parameter: $B_{\text {crit }}=\frac{B_{12}^{2}}{B_{11} B_{22}}>1$ (Ersch et al., 2016). The parameter is a measure for the compatibility between the two components. The higher the value, the more incompatible they are. The binary mixture $A B$ has a $B_{\text {crit }}$ of 6.76 . For mixtures with more than two components we have to find another criterion to check for the possibility of two or three phase separation. We will therefore first investigate a number of different mixtures depending on the interactions in the binary sub-mixtures. We start with mixtures (section Section 4.3.1) for which the component $C$ does not show any phase separation at concentrations $\eta<0.5$ with $A$ or $B$. In this case $C$ shows thermodynamic compatibility with both $A$ and $B$. In the next section (Section 4.3.2), $C$ is incompatible with $A$ or $B$. The binary mixture $A C$ or $B C$ will phase separate and have a critical point at concentrations $\eta<0.5$. In the third section (Section 4.3.3), $C$ is incompatible with both $A$ and $B$. The binary mixtures $A C$ and $B C$ both have a critical point at concentrations $\eta<0.5$. For this type of mixtures we 
hypothesize that it becomes possible that the mixture will phase separate into more than two phases inspired the hypothesis put forward by the work of Mace et al. (2012), who report on multiphase systems containing polymers and surfactants.

\subsubsection{Compatible with both $A$ and $B$}

In the first mixture (Figure 4.3) we set the non-additivity parameter for both the interactions with $C$ to $-0.1\left(\Delta_{A C}=-0.1\right.$ and $\left.\Delta_{B C}=-0.1\right)$. In this case the sub-mixture $B C$ will not demix at any concentration, because $B_{\text {crit }_{B C}}<1$ (see Table 4.1); the size difference between $B$ and $C$ combined with the negative non-additivity is not enough for phase separation. The sub-mixture $A C$ can theoretically phase separate $\left(B_{\text {crit }_{A C}}>1\right)$, however the critical point is at unattainable high concentrations $\left(\eta_{\text {crit }}>1\right)$. For the ternary mixture, we see that the addition of the third component $C$ to the binary mixture $A B$ has little effect on the phase behavior of the two components. With increasing concentrations of $C$ the plait points shift to slightly higher concentrations of $B$ and the spinodal shifts towards lower concentrations of $B$ at higher concentrations of $A$. There is no difference in the position of the binodal. With increasing concentration of $C$, the two phases will have the same volume fraction $(\alpha)$ and the same demixing of $A$ and $B$, independent of $C$ (see also Figure 4.B.1c). Component $C$ is present in both phases with concentrations similar to the concentration in the parent phase. For this system the tielines show very little rotation, so they are relatively parallel to the $x y$-plane.

For the next mixture (Figure 4.4) we kept the interaction between $A$ and $C$ the same, but we increased the non-additivity parameter between $B$ and $C$ to hard sphere interaction $\left(\Delta_{A C}=-0.1\right.$ and $\Delta_{B C}=0$ ). Now it is theoretically possible for $B$ and $C$ to phase separate $\left(B_{\text {crit }_{B C}}>1\right)$ (see Table 4.1), however just as for $A$ and $C$ the critical point is at unattainably high concentrations. For this mixture we see an increased shift in the position of the plait points, again towards higher concentrations for $B$ with an increase in concentration of $C$. For this mixture, the binodal also starts to shift towards slightly lower concentrations of $A$ at higher concentrations of $C$.

The fact that the plait points shift to different concentrations of $A$ and $B$ with the addition of the third component $C$, is an indication that the volumes of both phases will be different for specific mixtures of $A$ and $B$ with the addition of $C$. Not only will the volumes change, also the preferential fractionation of the components in both phases will be different. The more the plait points shift to different concentrations, the more the tie-lines between phases will show rotation with the addition of the third component. This can be seen in Figure 4.B.2c, where component $C$ has a preference of going to the top phase (phase enriched in $A$ ) and the volume fraction $(\alpha)$ of the bottom phase (phase enriched in 


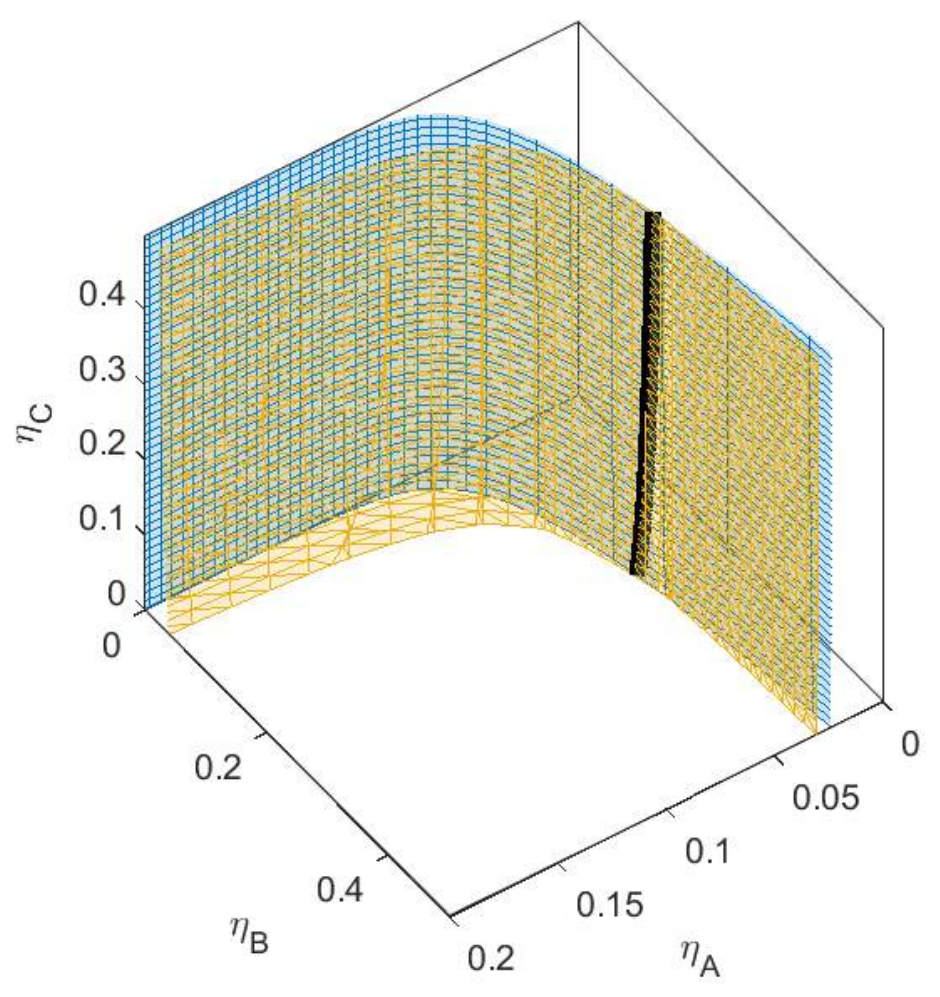

(a) 3D ternary plot

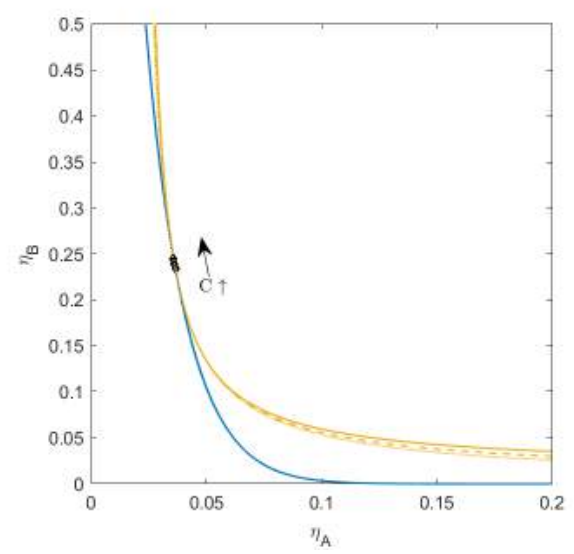

(b) Cross-sections

Figure 4.3: (a) Phase diagram for monodisperse ternary (component $A, B$, and $C$ ) non-additive hard sphere mixture with size ratio $q_{A B}=\sigma_{A} / \sigma_{B}=1 / 4$ and $q_{A C}=\sigma_{A} / \sigma_{C}=1 / 3$, with nonadditivity parameters: $\Delta_{A B}=0.1, \Delta_{A C}=-0.1$, and $\Delta_{B C}=-0.1$, plotted as a function of the partial packing fractions, $\eta_{A}, \eta_{B}$, and $\eta_{C}$. The spinodal (yellow surface) and binodal (blue surface) meet each other at the plait point-line (black line), (b) Phase diagram of component $A$ and $B$ at specific concentrations of component $C$, yellow lines are the spinodal, blue lines are the binodal, and the black diamonds are the critical points. Full line at concentration $\eta_{C}=0.05$, dashed line at concentration $\eta_{C}=0.15$, and dotted line at concentration $\eta_{C}=0.30$, with increasing concentration of $C$, the critical point shifts to higher concentrations of $B$. 


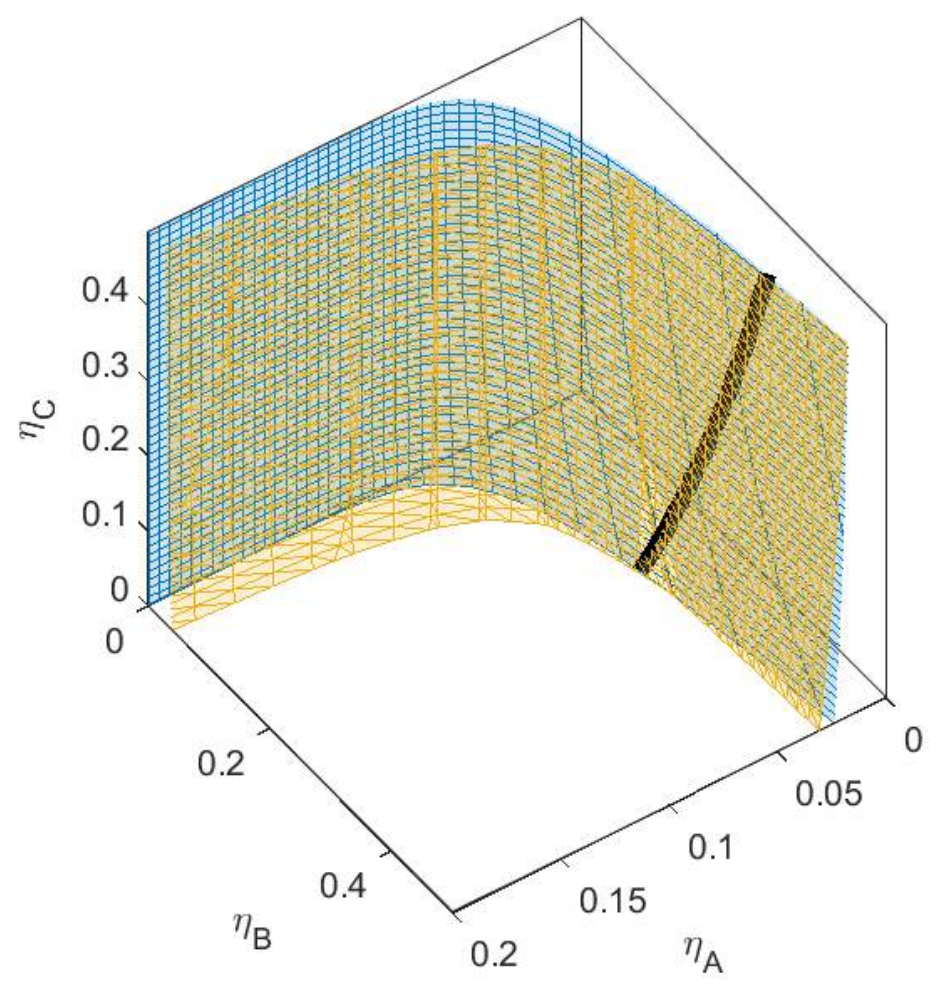

(a) 3D ternary plot

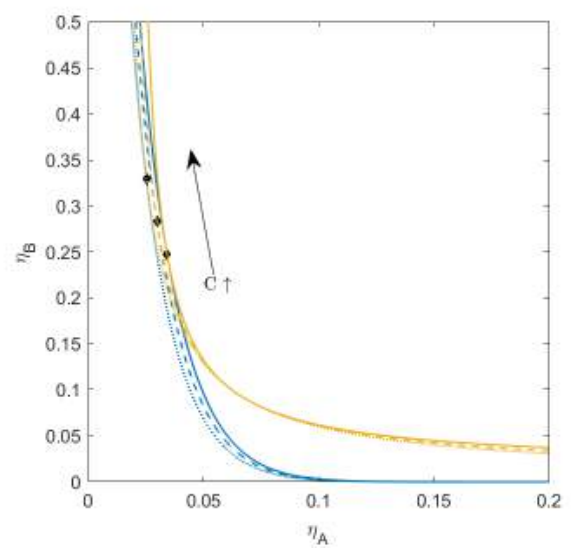

(b) Cross-sections

Figure 4.4: (a) Phase diagram for monodisperse ternary (component $A, B$, and $C$ ) non-additive hard sphere mixture with size ratio $q_{A B}=\sigma_{A} / \sigma_{B}=1 / 4$ and $q_{A C}=\sigma_{A} / \sigma_{C}=1 / 3$, with nonadditivity parameters: $\Delta_{A B}=0.1, \Delta_{A C}=-0.1$, and $\Delta_{B C}=0$, plotted as a function of the partial packing fractions, $\eta_{A}, \eta_{B}$, and $\eta_{C}$. The spinodal (yellow surface) and binodal (blue surface) meet each other at the plait point-line (black line), (b) Phase diagram of component $A$ and $B$ at specific concentrations of component $C$, yellow lines are the spinodal, blue lines are the binodal, and the black diamonds are the critical points. Full line at concentration $\eta_{C}=0.05$, dashed line at concentration $\eta_{C}=0.15$, and dotted line at concentration $\eta_{C}=0.30$, with increasing concentration of $C$, the critical point shifts to higher concentrations of $B$. 
$B$ ) decrease slightly with an increased addition of $C$. The tielines in this system start to show some rotation.

\subsection{2 $C$ Compatible with $A$ or $B$ and incompatible with the other one}

For the mixture in Figure $\mathbf{4 . 5}$ we switched the non-additivity parameters from the previous mixture to: $\Delta_{A C}=0$ and $\Delta_{B C}=-0.1$. The sub-mixture $A C$ can phase separate at concentrations $\eta<0.5$, but the sub-mixture $B C$ cannot demix at all (see also Table 4.1). For this three component mixture, we see that the binodal-surface at $\eta_{B}=0$ intersects with the $x z$-plane, indicating phase separation between the binary mixture of $A$ and $C$. The mixture yields a plait-point line that has broken up into two sections. These sections meet at very high concentrations of $C$. In the cross sections (Figure 4.5b), we see that the plait points, as well as the spinodal at high concentrations of $A$, shift towards lower concentrations of $B$ with increasing concentration of $C$. There is little difference in the phase separation boundary for an increasing concentration of $C$, however the tielines do rotate, one end being at slightly lower concentrations of $C$, the other end at higher concentrations of $C$ compared to the parent phase.

In the mixture in Figure 4.6 the interactions between both $A$ and $C$, and $B$ and $C$ is additive hard sphere interaction $\left(\Delta_{A C}=\Delta_{B C}=0\right)$. The sub-mixture $A C$ can phase separate at concentrations $\eta<0.5$, but the sub-mixture $B C$ cannot demix at this low concentration, because the size difference between $B$ and $C$ is not large enough (see also Table 4.1). For this three component mixture, we see that the binodal-surface at $\eta_{B}=0$ intersects with the $x z$-plane, indicating phase separation between $A$ and $C$, just as in the previous mixture. The mixture has two sections of plait-point line, again just as the previous mixture, here however the plait point line first bends away from the short section, to meet the other section at very high concentrations of $C$. In the cross-sections (Figure 4.6b), we see that the plait points shift towards higher concentrations of $B$ with increasing concentration of $C$ and the spinodal at high concentrations of $A$ shifts towards lower concentrations of $B$.

For the next mixture (Figure 4.7), the non-additivity parameters were set to $\Delta_{A C}=0.1$ and $\Delta_{B C}=-0.1$. Due to the increased incompatibility between $A$ and $C$, compared to Figure 4.5, the sections of plait pint line meet each other at lower concentrations. In the cross-sections (Figure $\mathbf{4 . 7 b}$ ), the cross-section at the highest concentration of $C$ intersects this line twice, leading to two plait-points at $\eta_{C}=0.30$. Because component $C$ has higher compatibility with $B$ compared to $A$, this component now preferentially goes to the bottom phase upon phase separation. With increased concentration of $C$, the volume fraction $(\alpha)$ of the bottom phase increases as well (see also Figure 4.B.5c), indicating the more 


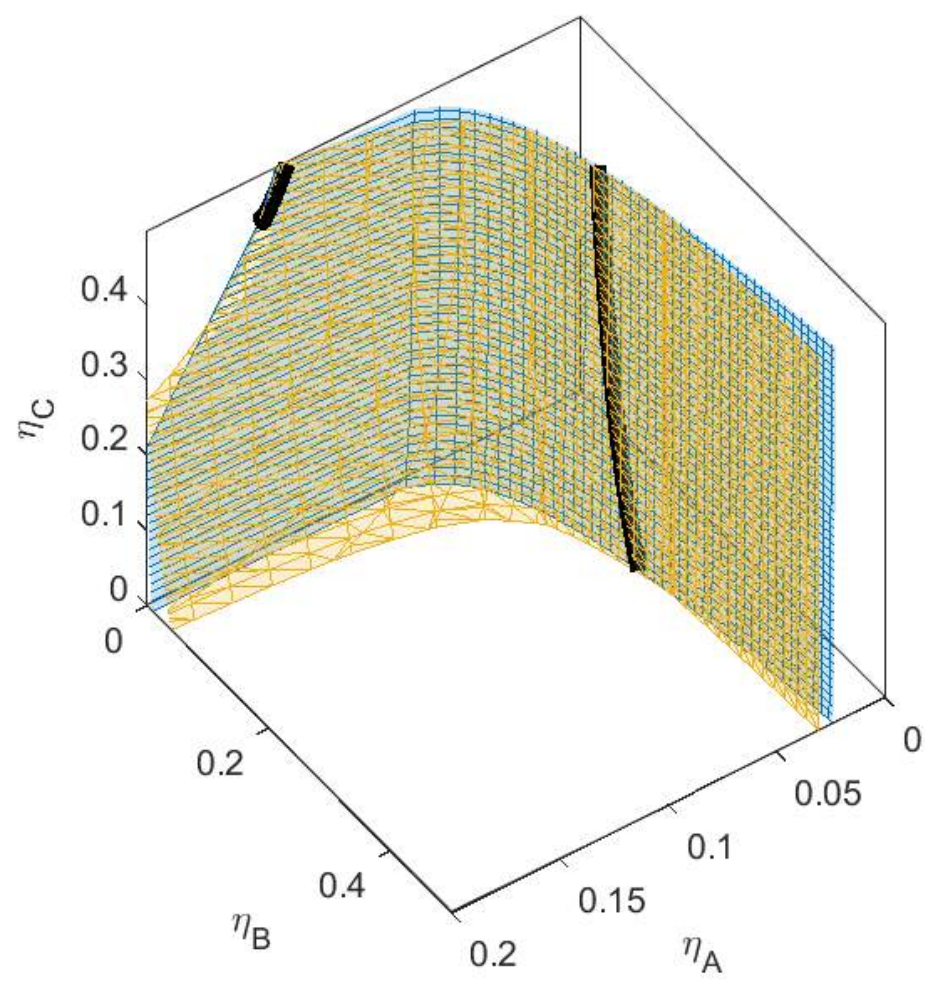

(a) 3D ternary plot

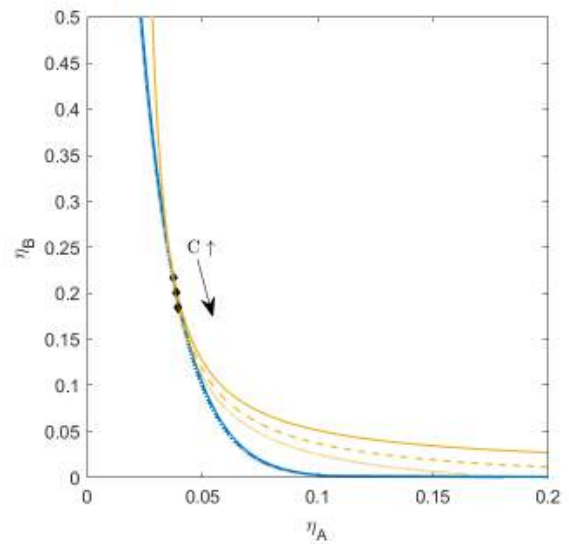

(b) Cross-sections

Figure 4.5: (a) Phase diagram for monodisperse ternary (component $A, B$, and $C$ ) non-additive hard sphere mixture with size ratio $q_{A B}=\sigma_{A} / \sigma_{B}=1 / 4$ and $q_{A C}=\sigma_{A} / \sigma_{C}=1 / 3$, with nonadditivity parameters: $\Delta_{A B}=0.1, \Delta_{A C}=0$, and $\Delta_{B C}=-0.1$, plotted as a function of the partial packing fractions, $\eta_{A}, \eta_{B}$, and $\eta_{C}$. The spinodal (yellow surface) and binodal (blue surface) meet each other at the plait point-line (black line), (b) Phase diagram of component $A$ and $B$ at specific concentrations of component $C$, yellow lines are the spinodal, blue lines are the binodal, and the black diamonds are the critical points. Full line at concentration $\eta_{C}=0.05$, dashed line at concentration $\eta_{C}=0.15$, and dotted line at concentration $\eta_{C}=0.30$, with increasing concentration of $C$, the critical point shifts to lower concentrations of $B$. 


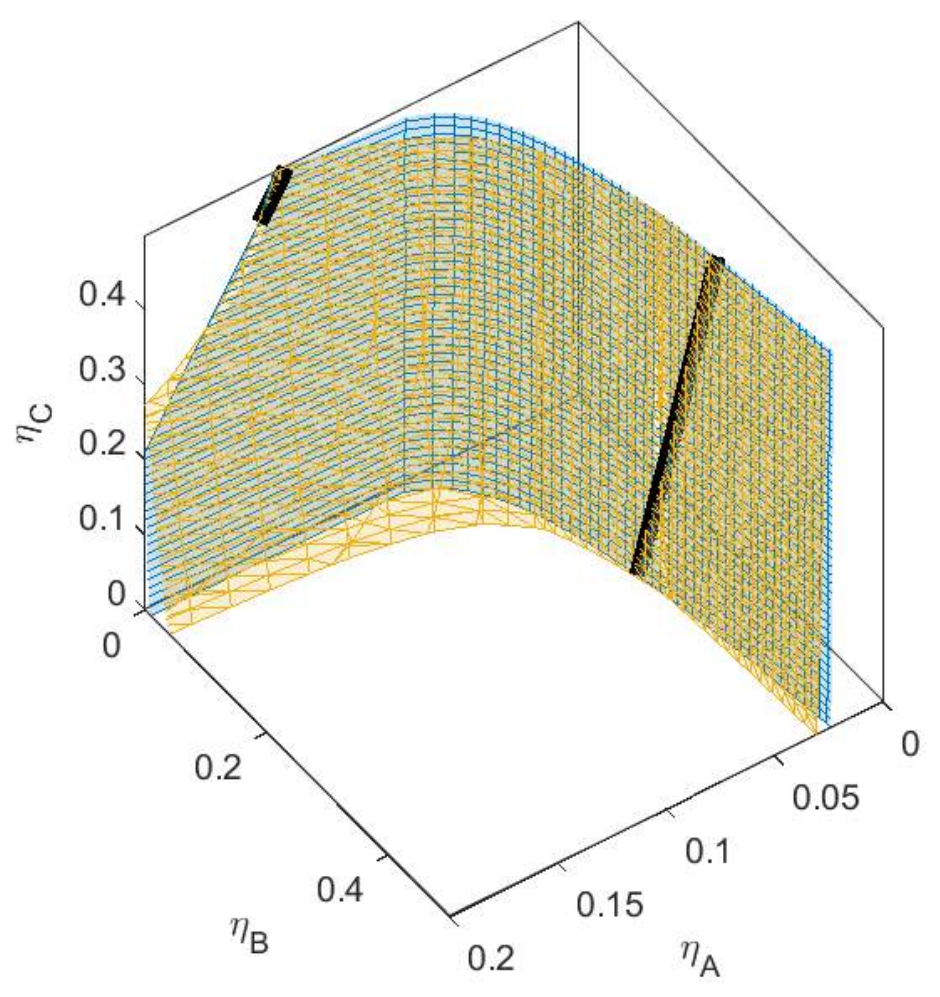

(a) 3D ternary plot

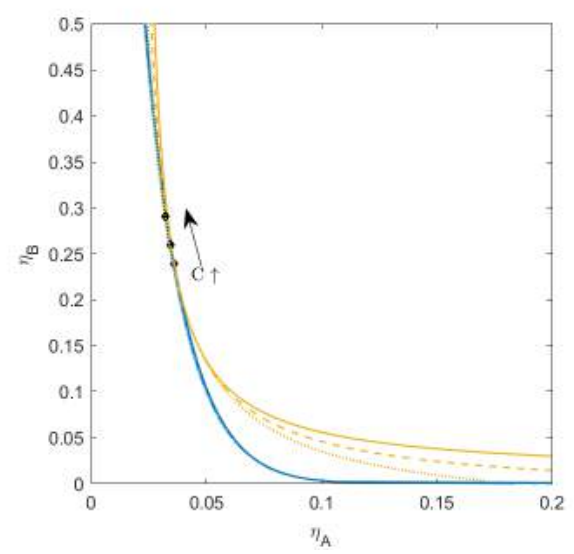

(b) Cross-sections

Figure 4.6: (a) Phase diagram for monodisperse ternary (component $A, B$, and $C$ ) non-additive hard sphere mixture with size ratio $q_{A B}=\sigma_{A} / \sigma_{B}=1 / 4$ and $q_{A C}=\sigma_{A} / \sigma_{C}=1 / 3$, with non-additivity parameters: $\Delta_{A B}=0.1, \Delta_{A C}=0$, and $\Delta_{B C}=0$, plotted as a function of the partial packing fractions, $\eta_{A}, \eta_{B}$, and $\eta_{C}$. The spinodal (yellow surface) and binodal (blue surface) meet each other at the plait point-line (black line), (b) Phase diagram of component $A$ and $B$ at specific concentrations of component $C$, yellow lines are the spinodal, blue lines are the binodal, and the black diamonds are the critical points. Full line at concentration $\eta_{C}=0.05$, dashed line at concentration $\eta_{C}=0.15$, and dotted line at concentration $\eta_{C}=0.30$, with increasing concentration of $C$, the critical point shifts to higher concentrations of $B$. 


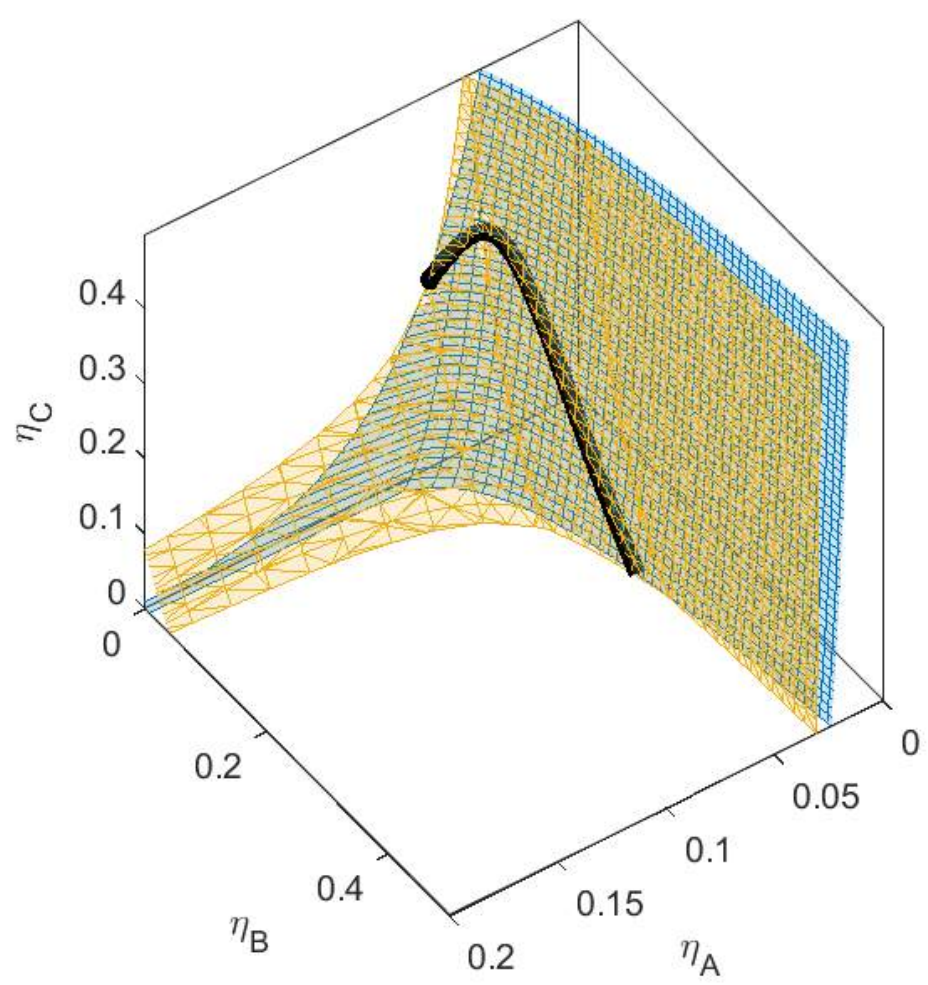

(a) 3D ternary plot

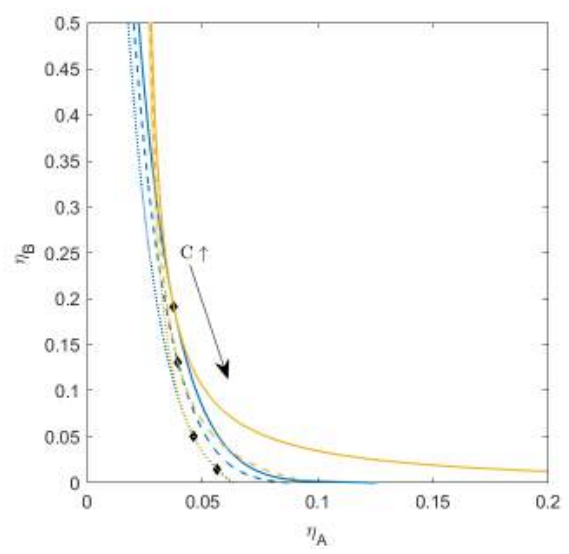

(b) Cross-sections

Figure 4.7: Phase diagram for monodisperse ternary (component $A, B$, and $C$ ) non-additive hard sphere mixture with size ratio $q_{A B}=\sigma_{A} / \sigma_{B}=1 / 4$ and $q_{A C}=\sigma_{A} / \sigma_{C}=1 / 3$, with non-additivity parameters: $\Delta_{A B}=0.1, \Delta_{A C}=0.1$, and $\Delta_{B C}=-0.1$, plotted as a function of the partial packing fractions, $\eta_{A}, \eta_{B}$, and $\eta_{C}$. The spinodal (yellow surface) and binodal (blue surface) meet each other at the plait point-line (black line), (b) Phase diagram of component $A$ and $B$ at specific concentrations of component $C$, yellow lines are the spinodal, blue lines are the binodal, and the black diamonds are the critical points. Full line at concentration $\eta_{C}=0.05$, dashed line at concentration $\eta_{C}=0.15$, and dotted line at concentration $\eta_{C}=0.30$, with increasing concentration of $C$, the critical point shifts to higher concentrations of $B$. 
pronounced rotation of the tielines connecting the child phases with the parent phase in a straight line.

With increased incompatibility between $B$ and $C\left(\Delta_{A C}=0.1\right.$ and $\left.\Delta_{B C}=0\right)$, the sections of plait point line meet each other at even lower concentrations (Figure 4.8). In the cross-sections (Figure $\mathbf{4 . 8 b}$ ), the cross-section at the highest concentration of $C$ does not have a plait point anymore. Component $C$ still has more affinity with component $B$ and prefers the bottom phase upon phase separation (Figure 4.B.6c), however due to the increased incompatibility between $B$ and $C$, the volume fraction $(\alpha)$ of the bottom phase increases more upon phase separation compared to the previous mixture.

In the mixture in Figure 4.9, $A$ and $C$ have a negative non-additivity parameter and $B$ and $C$ have a positive non-additivity parameter $\left(\Delta_{A C}=-0.1\right.$ and $\left.\Delta_{B C}=0.1\right)$. The sub-mixture $A C$ does not have a critical point at low enough concentrations to phase separate, however the sub-mixture $B C$ does phase separate. For the ternary mixture, the plait points shifts towards higher concentrations of $B$ with increasing concentration of $C$, until the critical point of the sub-mixture $B C$ is reached. With decreasing concentration of $A$, the binodal and spinodal bend towards the $y z$-plane, until the surfaces meet with

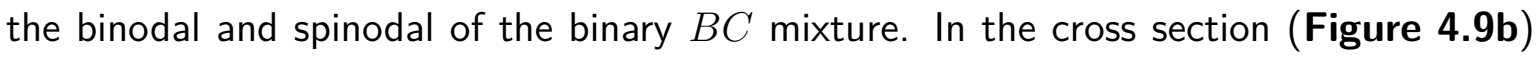
this can be seen as with increasing concentrations of $C$, the binodal shifts towards lower concentrations of $A$. Component $C$ is most compatible with component $A$, therefore upon phase separation component $C$ preference the top phase. With increased concentration of $C$, the volume fraction $(\alpha)$ of the top phase increases (see also Figure 4.B.7c).

\subsection{3 $C$ Incompatible with both $A$ and $B$}

The three sub-mixtures of the mixture in Figure 4.10 (with $\Delta_{A C}=0$ and $\Delta_{B C}=0.1$ ) all show phase separation as can be seen from both the binodal and the spinodal surfaces shifting towards the $x z$-plane and $y z$-plane (see also Table 4.1). We hypothesized that this type of mixture can demix into three phases, however as can be seen in the graph, this mixture does not show three-phase separation. The lowest concentration of the three-phase boundary is at concentrations $\eta>1$ and therefore unattainable. There are again two sections of plait point lines for this mixture, just as in some of the mixtures in the previous section. These two sections of plait point lines do not meet each other, not even at very high concentrations of $C$. In the cross-sections, Figure $4.10 \mathrm{~b}$, we see that with increasing concentration of component $C$, the plait point shifts to lower concentrations of $A$ and higher concentrations of $B$.

The three sub-mixtures of the mixture in Figure 4.11 (with $\Delta_{A C}=0.1$ and $\Delta_{B C}=0.1$ ) all show phase separation. For this mixture both the binodal and the spinodal surfaces 


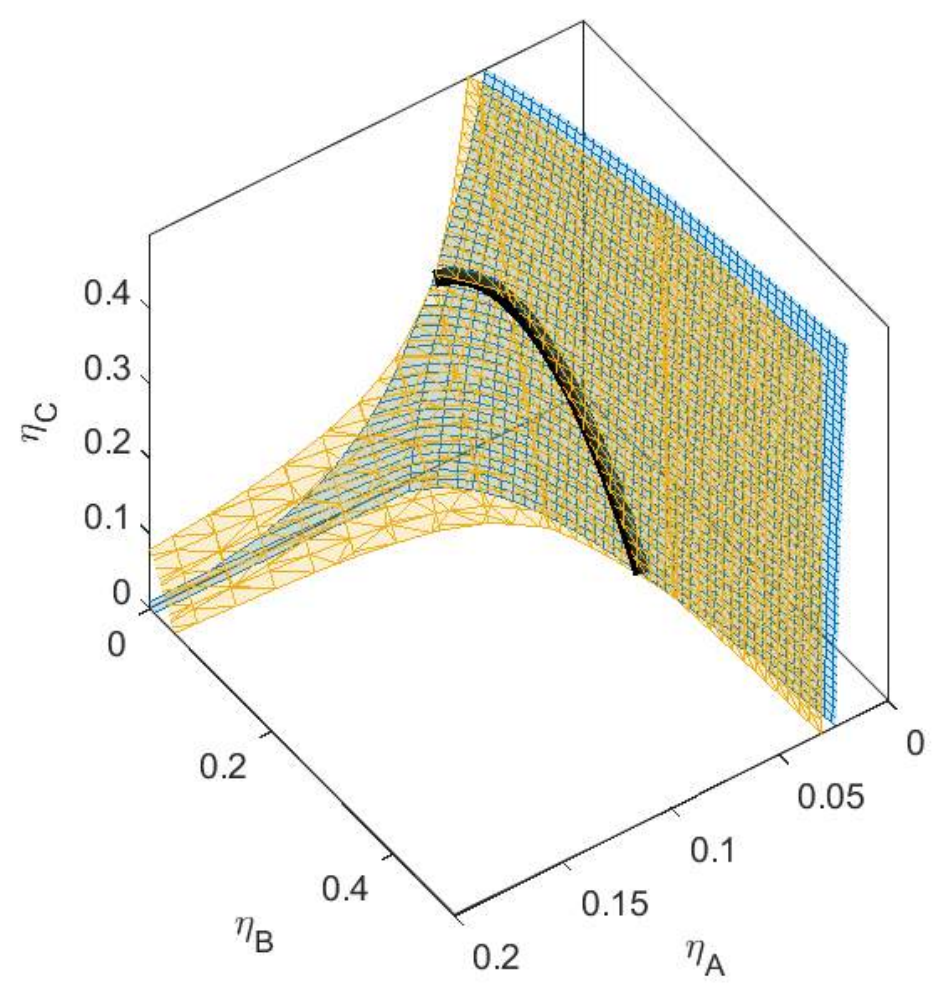

(a) 3D ternary plot

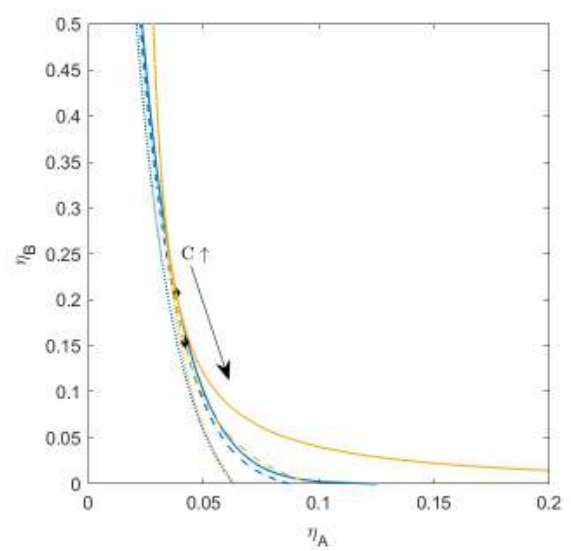

(b) Cross-sections

Figure 4.8: Phase diagram for monodisperse ternary (component $A, B$, and $C$ ) non-additive hard sphere mixture with size ratio $q_{A B}=\sigma_{A} / \sigma_{B}=1 / 4$ and $q_{A C}=\sigma_{A} / \sigma_{C}=1 / 3$, with non-additivity parameters: $\Delta_{A B}=0.1, \Delta_{A C}=0.1$, and $\Delta_{B C}=0$, plotted as a function of the partial packing fractions, $\eta_{A}, \eta_{B}$, and $\eta_{C}$. The spinodal (yellow surface) and binodal (blue surface) meet each other at the plait point-line (black line), (b) Phase diagram of component $A$ and $B$ at specific concentrations of component $C$, yellow lines are the spinodal, blue lines are the binodal, and the black diamonds are the critical points. Full line at concentration $\eta_{C}=0.05$, dashed line at concentration $\eta_{C}=0.15$, and dotted line at concentration $\eta_{C}=0.30$, with increasing concentration of $C$, the critical point shifts to higher concentrations of $B$. 


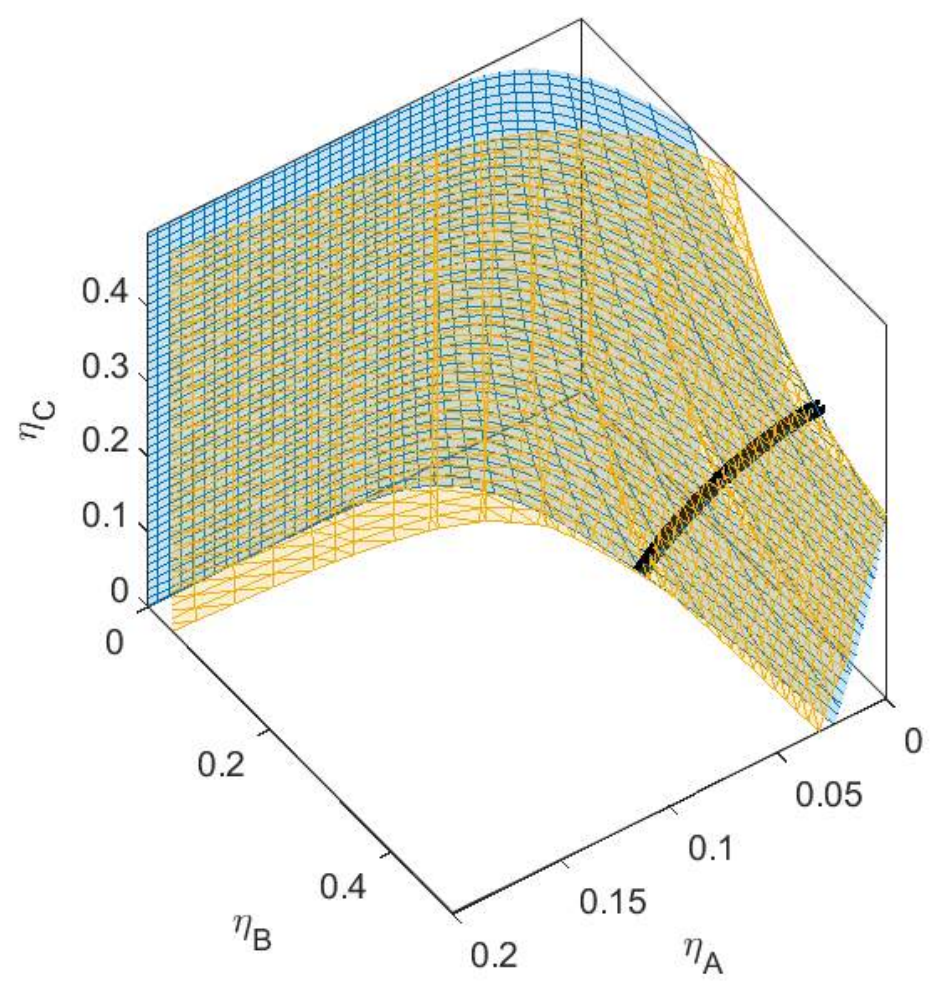

(a) 3D ternary plot

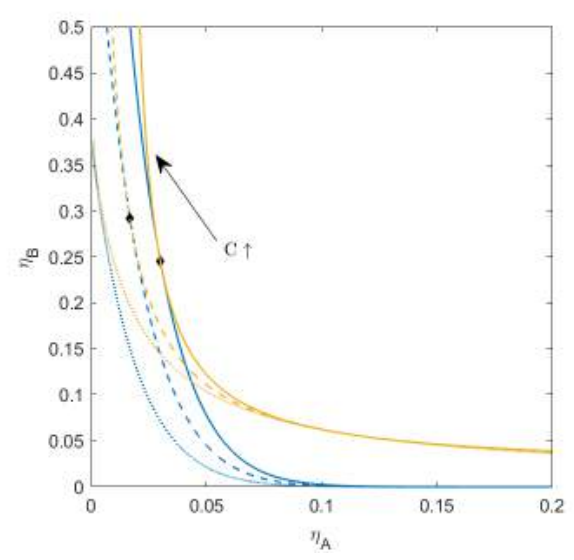

(b) Cross-sections

Figure 4.9: (a) Phase diagram for monodisperse ternary (component $A, B$, and $C$ ) non-additive hard sphere mixture with size ratio $q_{A B}=\sigma_{A} / \sigma_{B}=1 / 4$ and $q_{A C}=\sigma_{A} / \sigma_{C}=1 / 3$, with nonadditivity parameters: $\Delta_{A B}=0.1, \Delta_{A C}=-0.1$, and $\Delta_{B C}=0.1$, plotted as a function of the partial packing fractions, $\eta_{A}, \eta_{B}$, and $\eta_{C}$. The spinodal (yellow surface) and binodal (blue surface) meet each other at the plait point-line (black line), (b) Phase diagram of component $A$ and $B$ at specific concentrations of component $C$, yellow lines are the spinodal, blue lines are the binodal, and the black diamonds are the critical points. Full line at concentration $\eta_{C}=0.05$, dashed line at concentration $\eta_{C}=0.15$, and dotted line at concentration $\eta_{C}=0.30$, with increasing concentration of $C$, the critical point shifts to higher concentrations of $B$. 


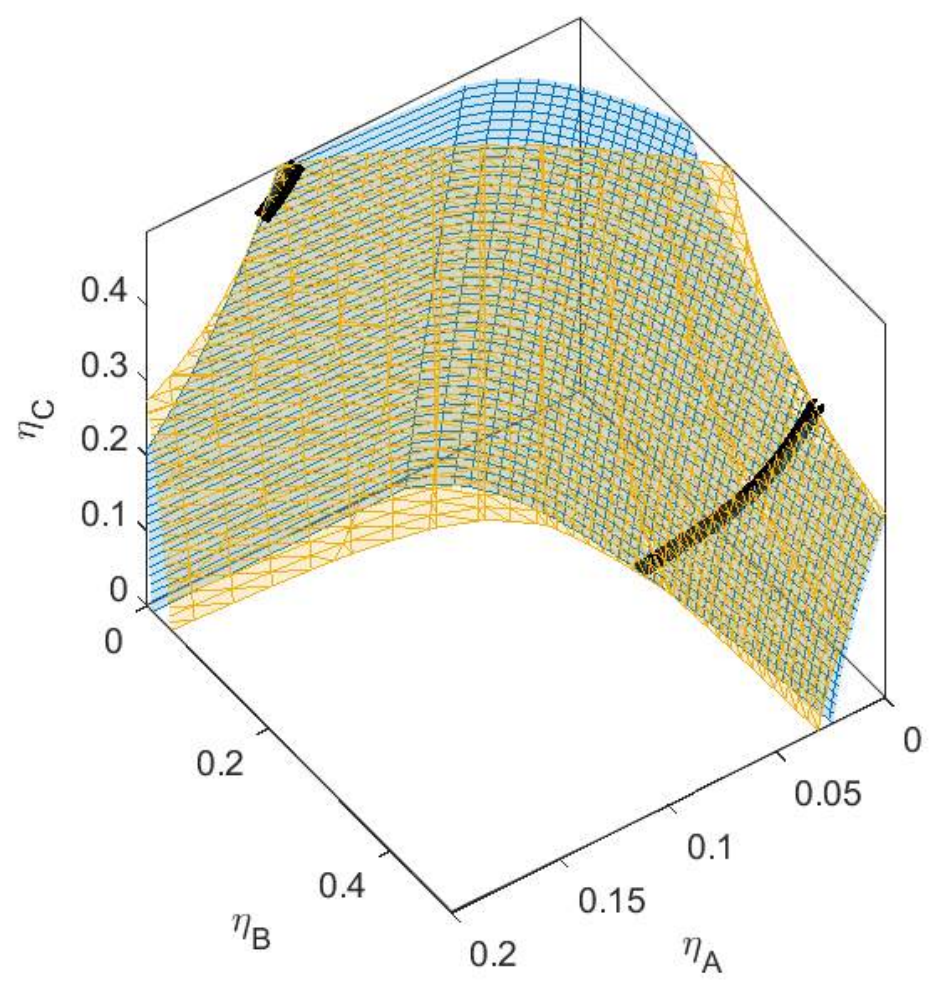

(a) 3D ternary plot

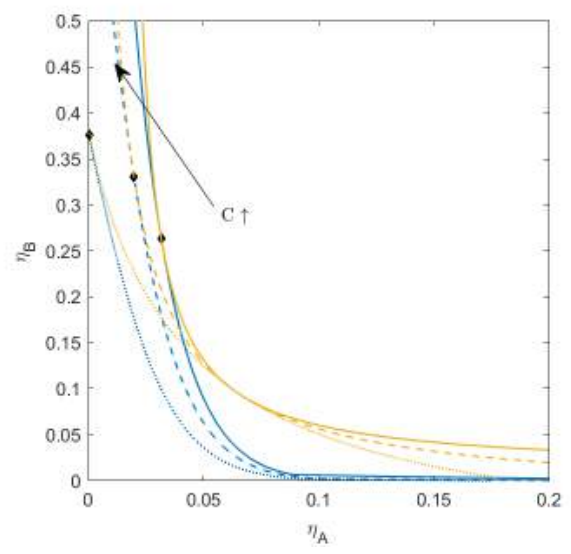

(b) Cross-sections

Figure 4.10: (a) Phase diagram for monodisperse ternary (component $A, B$, and $C$ ) non-additive hard sphere mixture with size ratio $q_{A B}=\sigma_{A} / \sigma_{B}=1 / 4$ and $q_{A C}=\sigma_{A} / \sigma_{C}=1 / 3$, with non-additivity parameters: $\Delta_{A B}=0.1, \Delta_{A C}=0$, and $\Delta_{B C}=0.1$, plotted as a function of the partial packing fractions, $\eta_{A}, \eta_{B}$, and $\eta_{C}$. The spinodal (yellow surface) and binodal (blue surface) meet each other at the plait point-line (black line), (b) Phase diagram of component $A$ and $B$ at specific concentrations of component $C$, yellow lines are the spinodal, blue lines are the binodal, and the black diamonds are the critical points. Full line at concentration $\eta_{C}=0.05$, dashed line at concentration $\eta_{C}=0.15$, and dotted line at concentration $\eta_{C}=0.30$, with increasing concentration of $C$, the critical point shifts to higher concentrations of $B$. 


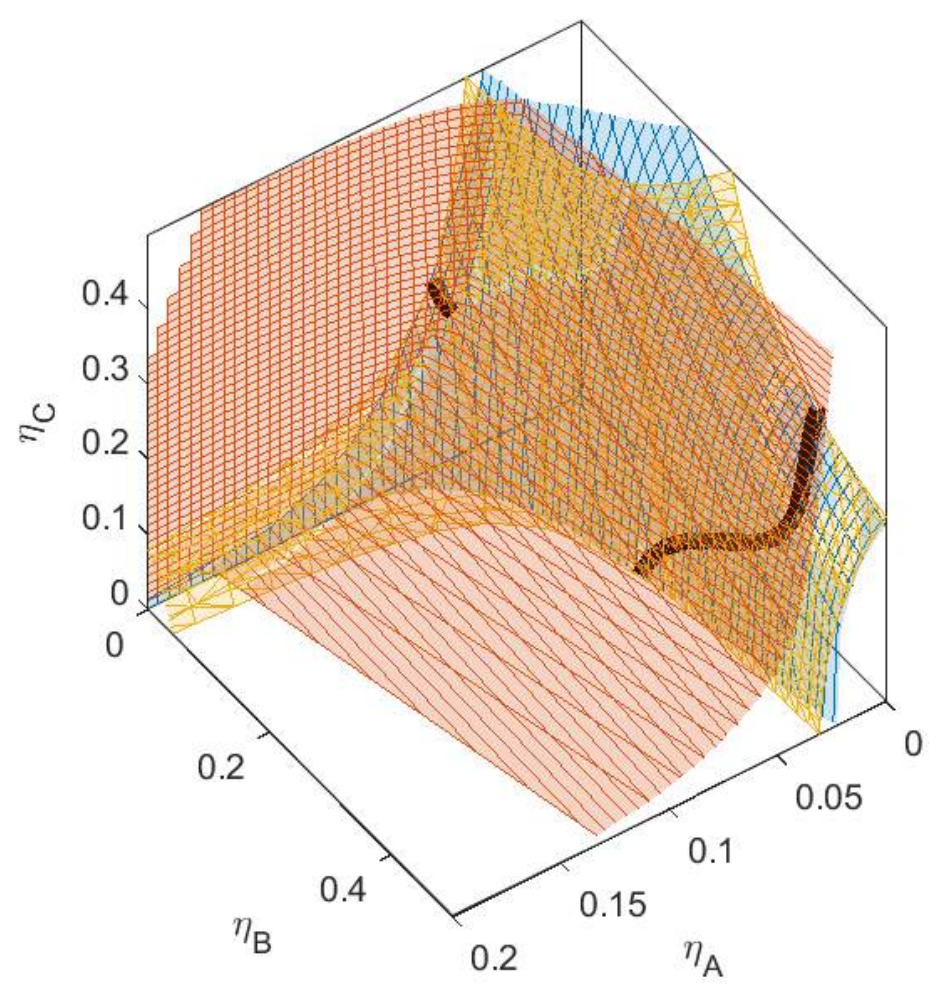

(a) 3D ternary plot

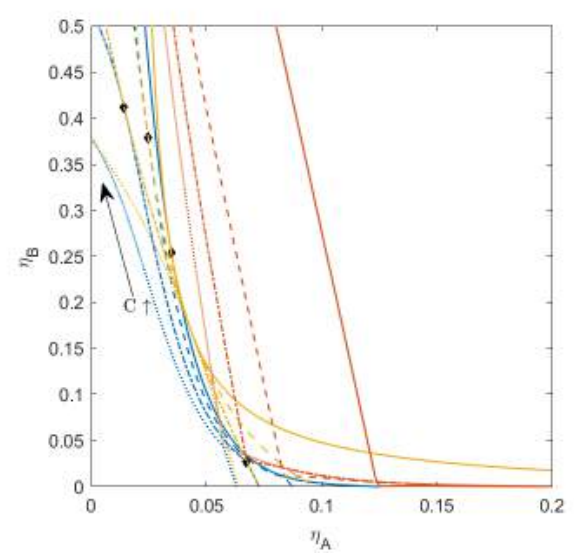

(b) Cross-ssections

Figure 4.11: (a) Phase diagram for monodisperse ternary (component $A, B$, and $C$ ) non-additive hard sphere mixture with size ratio $q_{A B}=\sigma_{A} / \sigma_{B}=1 / 4$ and $q_{A C}=\sigma_{A} / \sigma_{C}=1 / 3$, with nonadditivity parameters: $\Delta_{A B}=0.1, \Delta_{A C}=0.1$, and $\Delta_{B C}=0.1$, plotted as a function of the partial packing fractions, $\eta_{A}, \eta_{B}$, and $\eta_{C}$. The spinodal (yellow surface) and binodal (blue surface) meet each other at the plait point-line (black line), the mixture also has a three phase boundary (red surface), (b) Phase diagram of component $A$ and $B$ at specific concentrations of component $C$, yellow lines are the spinodal, blue lines are the binodal, red lines are the three phase boundary, and the black diamonds are the critical points. Full line at concentration $\eta_{C}=0.05$, dashed line at concentration $\eta_{C}=0.15$, dashed dotted line at concentration $\eta_{C}=0.227$, and dotted line at concentration $\eta_{C}=0.30$. 
Table 4.1: Virial coefficient ratios $\left(B_{\text {crit }}\right)$ for binary mixtures with $C$ depending on the nonadditivity parameter $\Delta$ and critical point for binary mixtures that phase separate (the interaction between $A$ and $B$ remains the same for all mixtures).

\begin{tabular}{lcccccc}
\hline Mixture & $\Delta_{A C}$ & $B_{c r i t} A C$ & $\eta_{c r i t} A C$ & $\Delta_{B C}$ & $B_{c_{r i t} B C}$ & $\eta_{\text {rrit }_{B C}}$ \\
\hline 4.3 & -0.10 & 1.26 & & -0.10 & 0.57 & \\
\hline 4.4 & -0.10 & 1.26 & & 0 & 1.06 & \\
\hline 4.5 & 0 & 2.37 & $(0.147,0.442)$ & -0.10 & 0.57 & \\
\hline 4.6 & 0 & 2.37 & $(0.147,0.442)$ & 0 & 1.06 & \\
\hline 4.7 & 0.10 & 4.20 & $(0.069,0.253)$ & -0.10 & 0.57 & \\
\hline 4.8 & 0.10 & 4.20 & $(0.069,0.253)$ & 0 & 1.06 & \\
\hline 4.9 & -0.10 & 1.26 & & 0.10 & 1.88 & $(0.386,0.295)$ \\
\hline 4.10 & 0 & 2.37 & $(0.147,0.442)$ & 0.10 & 1.88 & $(0.386,0.295)$ \\
\hline 4.11 & 0.10 & 4.20 & $(0.069,0.253)$ & 0.10 & 1.88 & $(0.386,0.295)$ \\
\hline
\end{tabular}

shift towards the $x z$-plane and $y z$-plane as well. In this case the incompatibility between all three components is large enough for the mixture to demix into three phases at low enough, physically relevant, concentrations. The lowest concentration of the three-phase boundary is at $\eta_{\text {crit }}=(0.067,0.028,0.227)$. This point is on the spinodal, the plait point line and also the binodal. This is a special plait point, where all three phases become indistinguishable. Small deviations in one of the concentrations of the three components will result in the formation of one homogeneous phase, two phases (depending on the perturbation, these phases are enriched in different components), or three phases. There are again two sections of plait point lines for this mixture, just as in some of the mixtures in the previous section. These two sections of plait point lines do not meet each other, not even at very high concentrations of $C$, since one section terminates at the three phase boundary. The two phase boundary has a bend where the surface meets with the three phase boundary. Depending on the concentrations of $A, B$, and $C$, the two phases will have very different fractionation.

\subsubsection{Fractionation}

In the previous sections we saw the phase diagrams of nine different mixtures for which we altered the interaction between a third component $C$ and two components $A$ and $B$ that phase separate. In this section we will qualitatively compare the phase behavior of the different mixtures at specific parent concentrations of the different components to get more insight into the phase separation dynamics. In all the fractionation figures, each component 


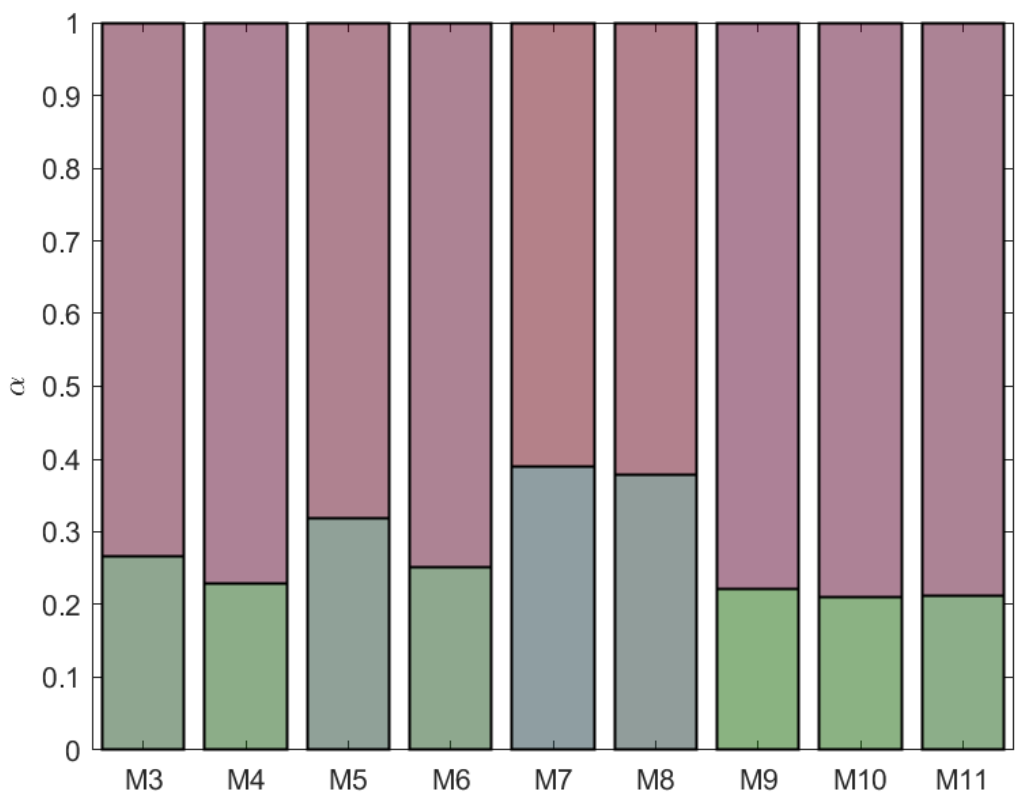

(a) $\eta(0.05,0.20,0.10)$

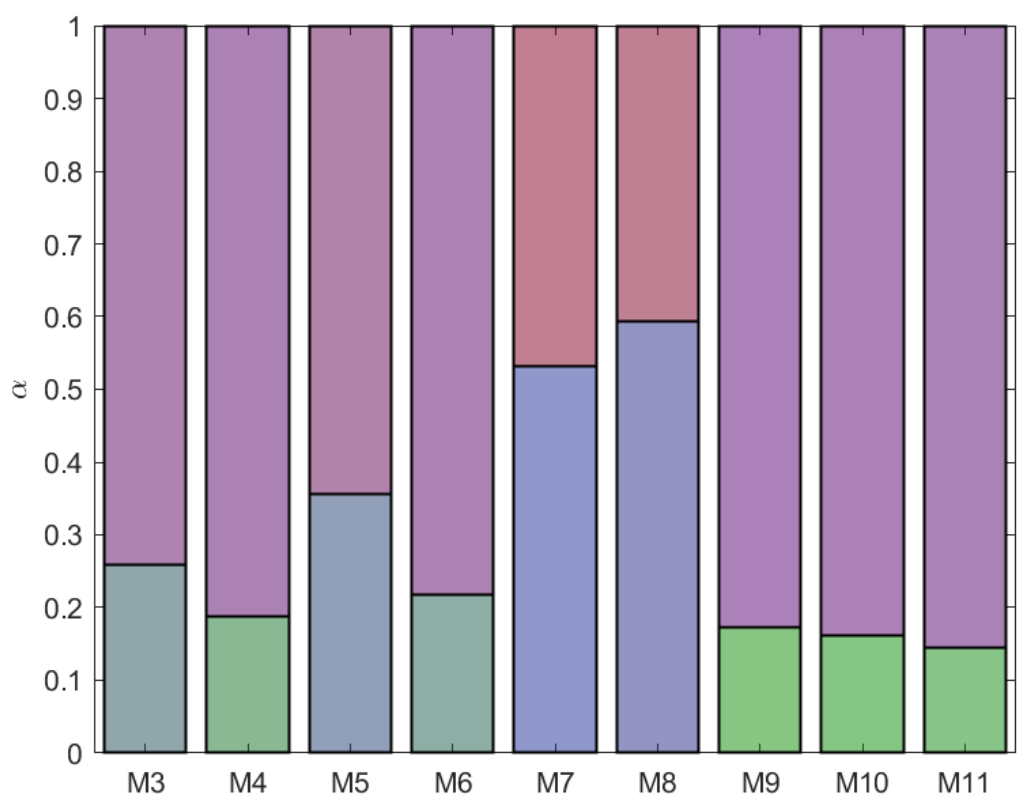

(b) $\eta(0.05,0.20,0.25)$

Figure 4.12: Fractionation of monodisperse ternary (component $A, B$, and $C$ ) non-additive hard sphere mixtures with size ratio $q_{A B}=\sigma_{A} / \sigma_{B}=1 / 4$ and $q_{A C}=\sigma_{A} / \sigma_{C}=1 / 3$, with non-additivity parameters: $\Delta_{A B}=0.1, \Delta_{A C}$ and $\Delta_{B C}$ varying from -0.1 to 0.1 , label referring to Figures 4.3 to 4.11 , at fixed parent phase, $A$ is red $\square, B$ is green $\square$, and $C$ is blue $\square$, table with concentrations in supplementary materials. 


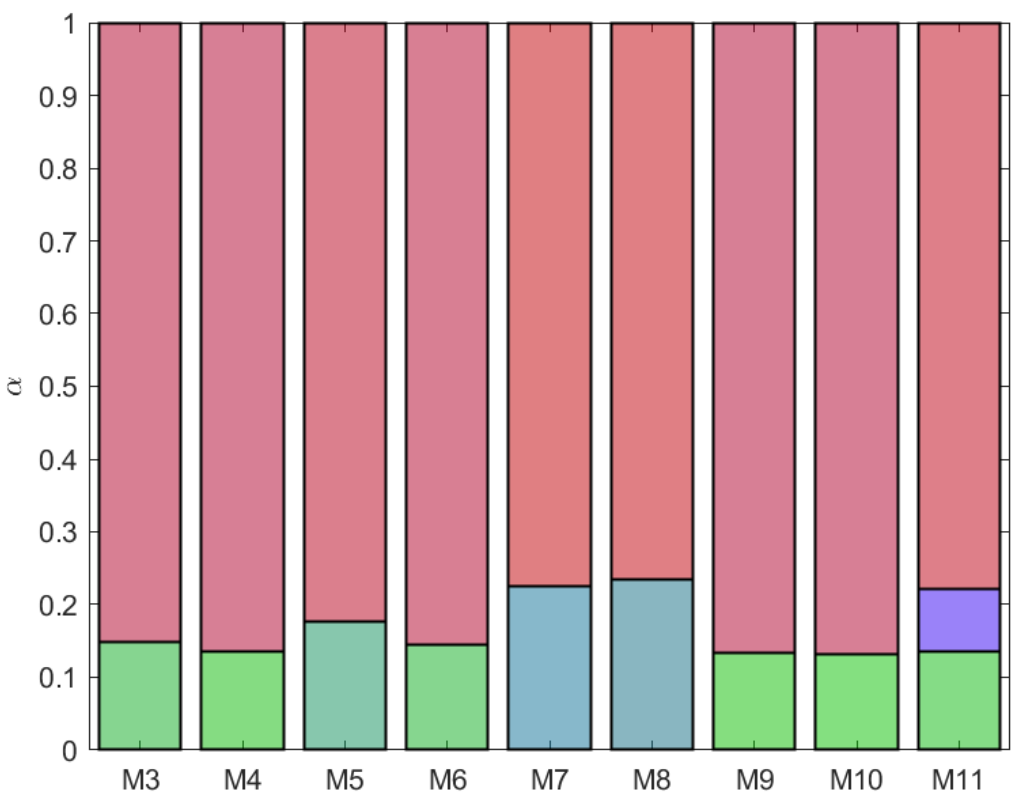

(a) $\eta(0.10,0.20,0.10)$

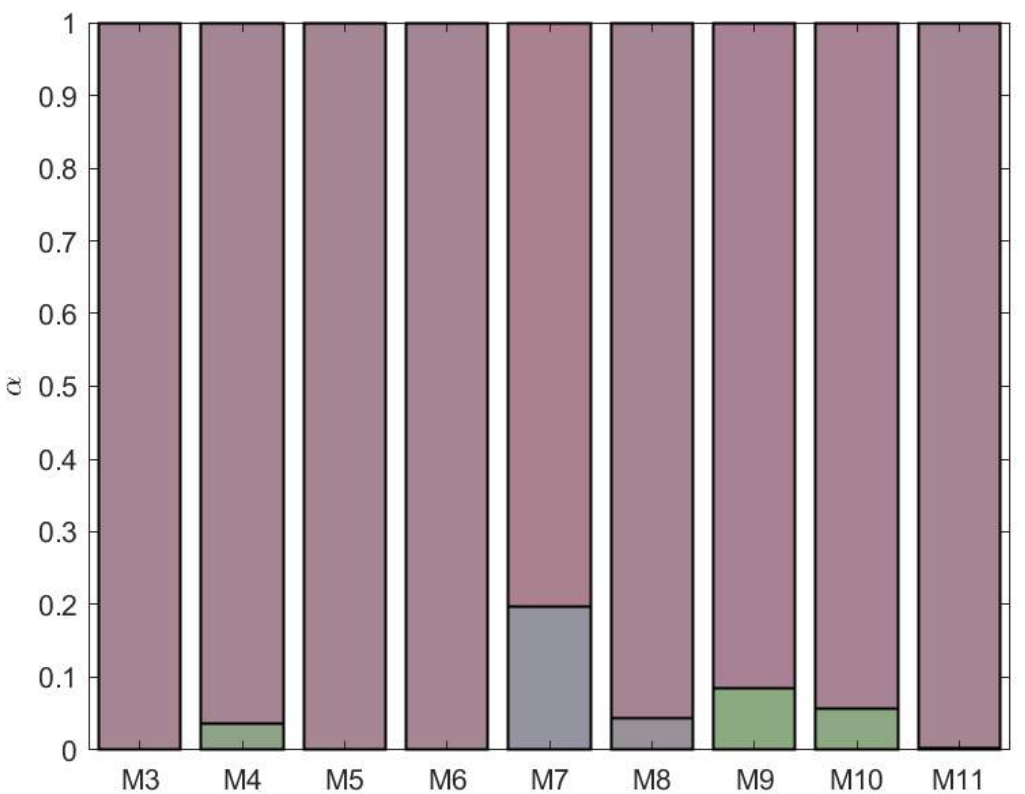

(b) $\eta(0.05,0.10,0.10)$

Figure 4.13: Fractionation of monodisperse ternary (component $A, B$, and $C$ ) non-additive hard sphere mixtures with size ratio $q_{A B}=\sigma_{A} / \sigma_{B}=1 / 4$ and $q_{A C}=\sigma_{A} / \sigma_{C}=1 / 3$, with non-additivity parameters: $\Delta_{A B}=0.1, \Delta_{A C}$ and $\Delta_{B C}$ varying from -0.1 to 0.1 , label referring to Figures 4.3 to 4.11 , at fixed parent phase, $A$ is red $\square, B$ is green $\square$, and $C$ is blue $\square$, table with concentrations in supplementary materials. 
has a different color: component $A$ is red, component $B$ is green, and component $C$ is blue (see supplementary materials for a table). Depending on the concentration of each component in each phase, the color in the figure will be a combination of the different colors. In Figure 4.12a the parent phase has a concentration of $\eta(0.05,0.20,0.10)$. For all mixtures, component $A$, being the smallest component in the mixture, preferentially goes to the top phase, while component $B$, the largest component in the system, remains at the bottom. The volume fraction of the bottom phase $(\alpha)$ is largest for the mixtures from Figures 4.5, 4.7 and 4.8. For these mixtures, $A$ and $C$ were more incompatible than $B$ and $C$, as can be seen in Table 4.1 from their $B_{\text {crit }}$ values. Component $C$ preferentially goes to the bottom phase (more blue in bottom phase compared to top phase) at this parent concentration and the volume fraction of the bottom phase correspondingly increases.

Looking at a lot higher concentrations of $C,(\eta(0.05,0.20,0.25)$, Figure 4.12b), we see that for most mixtures component $C$ prefers the top phase, but for the two mixtures with the highest incompatibility between $A$ and $C$ and low to moderate incompatibility between $B$ and $C$ (the mixtures from Figures 4.7 and 4.8), clearly component $C$ moves to the bottom phase. The higher the concentration of $C$ in a phase, the more the volume fraction $\alpha$ shifts towards this phase. The bottom phase of the mixture from Figure 4.8 is the largest, whilst the bottom phase of the mixture from Figure 4.11 is the smallest.

When increasing the concentration of component $A$ on the other hand $(\eta(0.10,0.20,0.10)$, Figure 4.13a), we see that for the mixture from Figure 4.11, a third phase appears. At the higher concentration of $A$, component $C$ demixes from the top phase forming the new middle phase.

Finally we look at a parent concentration with a lower amount of $B(\eta(0.05,0.10,0.10)$, Figure 4.13b). At this concentration the mixture $A B$ does not show phase separation yet. However due to the addition of component $C$ some of the mixtures demix at this parent concentration. This is especially true for the mixtures with more compatibility between $B$ and $C$ and higher incompatibility between $A$ and $C$, the mixtures from Figures 4.7 and 4.8. The mixture from Figure $\mathbf{4 . 1 1}$ has the same incompatibility between $A$ and $C$, however due to the increased incompatibility between $B$ and $C$, the mixture remains stabilized. When a mixture demixes into two or more phases, the concentration of one or more components is higher in one of the phases compared to the other, while each of the phases has a smaller volume compared to the parent volume. Systems with less incompatibility among some of the components tend to demix at slightly lower concentration, compared to systems with high incompatibility. This was also shown experimentally by Johansson and Walter (1999) for the system polyethylene glycol and dextran, where a third polymer added to a dilute one phase mixture of polyethylene glycol and dextran caused the system to demix into two phases. At low concentrations, this third polymer distributes between the two phases. 


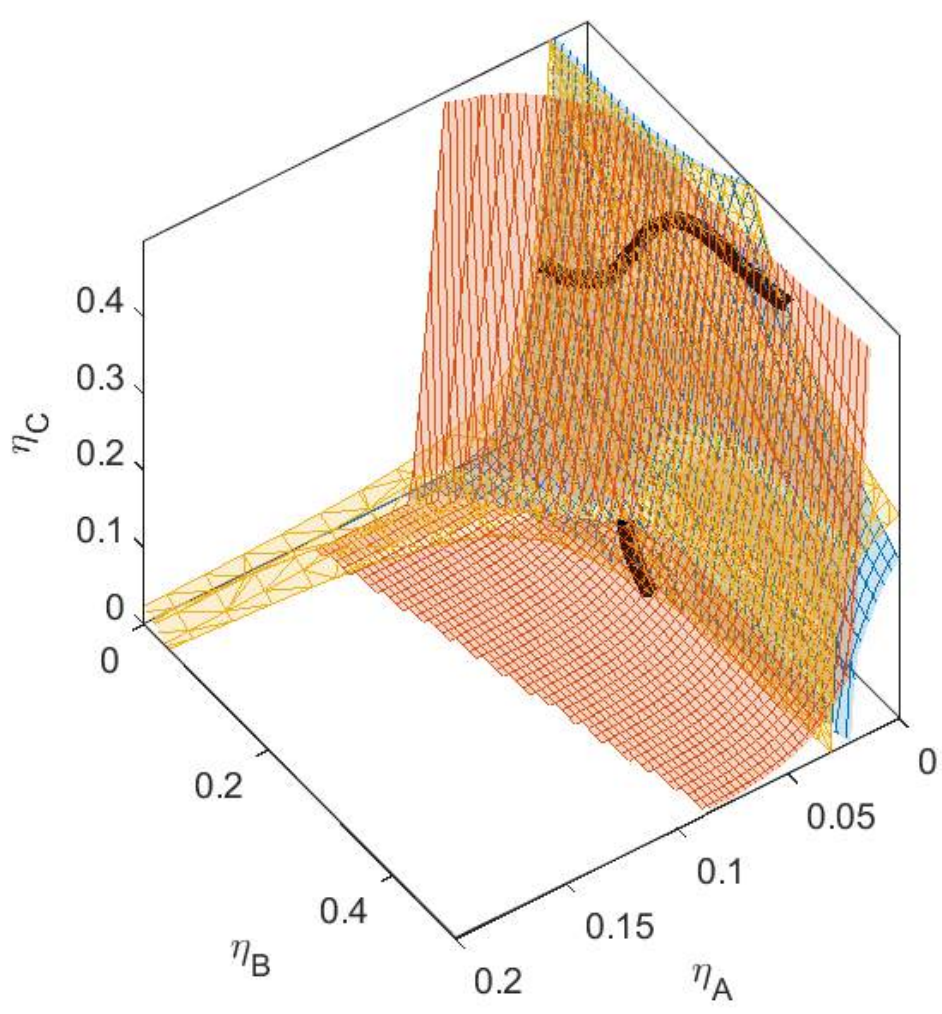

Figure 4.14: (a) Phase diagram for monodisperse ternary (component $A, B$, and $C$ ) non-additive hard sphere mixture with size ratio $q_{A B}=\sigma_{A} / \sigma_{B}=1 / 4$ and $q_{A C}=\sigma_{A} / \sigma_{C}=1 / 5$, with non-additivity parameters: $\Delta_{A B}=0.1, \Delta_{A C}=0.1$, and $\Delta_{B C}=0.1$, plotted as a function of the partial packing fractions, $\eta_{A}, \eta_{B}$, and $\eta_{C}$. The spinodal (yellow surface) and binodal (blue surface) meet each other at the plait point-line (black line), the mixture also has a three phase boundary (red surface).

See also AppendixSection B for the phase separated sequences of increasing concentration of components $A, B$ and $C$ in the parent phases for the mixtures from Figures 4.3 to 4.11 .

\subsubsection{Three-phase systems}

In the previous sections we saw one three-phase system. We changed the size of $C$ and the non-additive interaction parameters $\Delta_{A C}$ and $\Delta_{B C}$ to investigate the dynamics in three-phase systems. First we changed the size ratio between $A$ and $C$. The mixture in Figure 4.14 the size ratio is $q_{A C}=1 / 5$, and the non-additive interaction between $C$ and the other components is: $\Delta_{A C}=0.1$, and $\Delta_{B C}=0.1$ (the same as for Figure 4.11). When looking at the $B_{\text {crit }}$ (see Table 4.2) we see that this change in size resulted in more repulsive interaction between $A$ and $C$, while $B_{\text {crit }_{B C}}$ remains more or less the same. This 
Table 4.2: Virial coefficient ratios $\left(B_{\text {crit }}\right)$ for binary mixtures with $C$ depending on the nonadditivity parameter $\Delta$ and size ratio of $A$ and $C$ and critical point for binary mixtures that phase separate (the interaction between $A$ and $B$ remains the same for all mixtures). All systems show three-phase separation at concentrations $\eta<0.5$.

\begin{tabular}{lccccccc}
\hline Mixture & $q_{A C}$ & $\Delta_{A C}$ & $B_{\text {crit }_{A C}}$ & $\eta_{\text {crit }_{A C}}$ & $\Delta_{B C}$ & $B_{\text {crit }_{B C}}$ & $\eta_{\text {crit }_{B C}}$ \\
\hline 4.11 & $1 / 3$ & 0.10 & 4.20 & $(0.069,0.253)$ & 0.10 & 1.88 & $(0.386,0.295)$ \\
\hline 4.14 & $1 / 5$ & 0.10 & 10.33 & $(0.022,0.219)$ & 0.10 & 1.84 & $(0.318,0.391)$ \\
\hline 4.15 & $1 / 3$ & 0.15 & 5.48 & $(0.052,0.208)$ & 0.10 & 1.88 & $(0.386,0.295)$ \\
\hline
\end{tabular}

can also be seen in the concentration of the critical points of their respective binary mixtures, e.g. for the mixture $B C$ the total volume fraction at the critical point remains more or less the same, however their respective fractions are flipped due to their flip in size ratio. Compared to Figure 4.11 the three-phase boundary is rotated. The critical point of the three-phase boundary is at $\eta(0.035,0.188,0.052)$. Since component $C$ is now the largest component in the system (and therefore the heaviest of the three, assuming the density of the components is larger than the density of the solution), this component preferentially goes to the bottom phase, component $B$ preferentially goes to the middle phase and component $A$ is most abundant in the top phase. When we navigate through the phase diagram on a straight line with increasing component $A$ while keeping the concentration of components $B$ and $C$ the same, the system first demixes into a large top phase enriched in component $A$ and $B$ and a small bottom phase enriched in component $C$. This is due to the higher incompatibility of $C$ with $A$ compared to the incompatibility between $A$ and $B$. When more of component $A$ is added, component $B$ demixes from the top phase, forming the middle phase.

In Figure 4.15 we used again the same size of $C$ as in the first series of mixtures $\left(q_{A C}=1 / 3\right)$ and we increased the incompatibility between $A$ and $C$. Compared to Figures 4.11 and 4.14 the three-phase boundary is rotated. The critical point of the threephase system is at $\eta(0.027,0.412,0.169)$. When gradually increasing the concentration of component $C$, the two phase system of one phase enriched in $A$ (the top phase) and the other phase enriched in $B$, when reaching the three-phase boundary, component $C$ demixes from the top phase, forming the middle phase enriched in component $C$.

Experimental three phase systems of for example the colloids dextran, ficoll, and polyethylene-glycol (Hartman et al., 1974) and dextran, polyethylene-glycol, and gelatin (unpublished work) show similar phase behavior. Depending on the concentrations of each component, the system will form 1 phase or demix into two or three phases, each phase enriched in one of the components. 


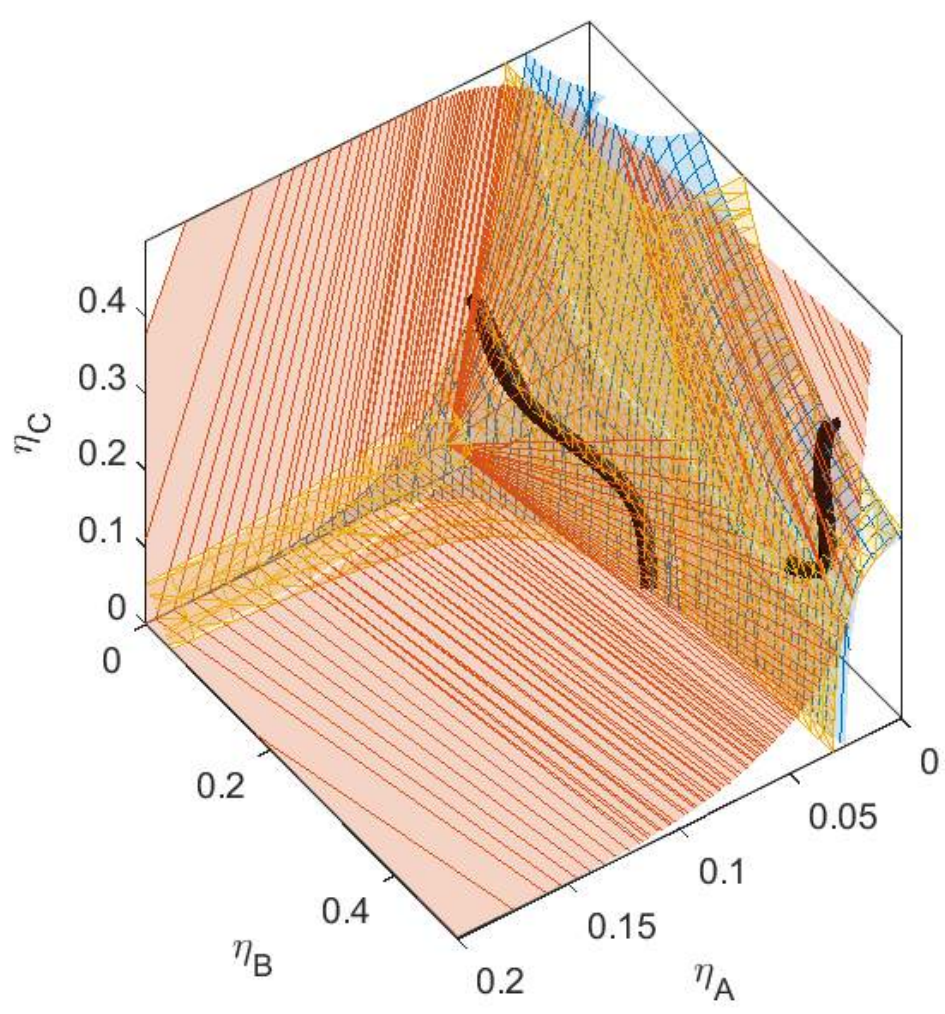

Figure 4.15: (a) Phase diagram for monodisperse ternary (component $A, B$, and $C$ ) non-additive hard sphere mixture with size ratio $q_{A B}=\sigma_{A} / \sigma_{B}=1 / 4$ and $q_{A C}=\sigma_{A} / \sigma_{C}=1 / 3$, with nonadditivity parameters: $\Delta_{A B}=0.1, \Delta_{A C}=0.15$, and $\Delta_{B C}=0.1$, plotted as a function of the partial packing fractions, $\eta_{A}, \eta_{B}$, and $\eta_{C}$. The spinodal (yellow surface) and binodal (blue surface) meet each other at the plait point-line (black line), the mixture also has a three phase boundary (red surface).

\subsection{Conclusion}

The addition of a third component $C$ to a binary mixture $A B$ that demixes has a different impact depending on the pairwise compatibility between the third components and the two other components. Depending on the pairwise interactions for the three components, the volume fractions and compositions of the three phases are altered. If the third component is compatible with both components, the phase boundary and spinodal are nearly vertical surfaces. The third component therefore then does not decrease the incompatibility between the components $A$ and $B$. The component $C$ is present in both phases in the same amount and the volume fraction between the phases is not altered by the addition of the third component. The plait point line of the mixture will shift to lower or higher concentrations of the components $A$ and $B$ with increasing concentration of $C$ depending on the interaction of $C$. The sum of the volume fractions of $A$ and $B$ is however always equal or lower compared 
to the sum of the volume fractions at the critical point of the binary mixture.

When $C$ is incompatible with one of the components $A$ or $B$, the binodal and spinodal will bend towards one of the planes (either the $x z$-plane or $y z$-plane) of the phase diagram. The plait point line forms a curve that connects the critical points of the binary mixtures that demix. When all three components are incompatible, it becomes possible for the mixture to demix into three phases. The lowest concentration of the three phase boundary, lays also on the spinodal and for some of the mixtures on the two phase boundary as well. For these mixtures, small perturbations in the concentrations of one the components at this critical point will result in either one homogeneous phase, two phases or three phases. Depending on the different pairwise interactions between the components, the three-phase boundary has a different shape and rotation. 


\subsection{Bibliography}

P.-Å. Albertsson and G. Birkenmeier. Affinity separation of proteins in aqueous threephase systems. Analytical Biochemistry, 175(1):154-161, 1988. ISSN 10960309. doi: 10.1016/0003-2697(88)90373-9.

S. Beck-Candanedo, D. Viet, and D. G. Gray. Triphase equilibria in cellulose nanocrystal suspensions containing neutral and charged macromolecules. Macromolecules, 40(9): 3429-3436, 2007. ISSN 00249297. doi: 10.1021/ma0704818.

B. L. Beegle, M. Modell, and R. C. Reid. Thermodynamic stability criterion for pure substances and mixtures. AIChE Journal, 20(6):1200-1206, 1974. ISSN 0001-1541. doi: 10.1002 /aic.690200621.

X. L. Chu, A. D. Nikolov, and D. T. Wasan. Effects of Particle Size and Polydispersity on the Depletion and Structural Forces in Colloidal Dispersions. Langmuir, 12(21):5004-5010, 1996. ISSN 0743-7463. doi: 10.1021/la960359u.

M. W. Edelman, E. van der Linden, E. de Hoog, and R. H. Tromp. Compatibility of Gelatin and Dextran in Aqueous Solution. Biomacromolecules, 2(4):1148-1154, 2001. ISSN 1525-7797. doi: 10.1021/bm015545f.

M. W. Edelman, E. Van Der Linden, and R. H. Tromp. Phase separation of aqueous mixtures of poly(ethylene oxide) and dextran. Macromolecules, 36(20):7783-7790, 2003. ISSN 00249297. doi: 10.1021/ma0341622.

C. Ersch, E. van der Linden, A. Martin, and P. Venema. Interactions in protein mixtures. Part II: A virial approach to predict phase behavior. Food Hydrocolloids, 52:991-1002, 2016. doi: 10.1016/j.foodhyd.2015.07.021.

T. S. Harmon, A. S. Holehouse, and R. V. Pappu. To Mix, or To Demix, That Is the Question. Biophysical Journal, 112(4):565-567, 2017. ISSN 15420086. doi: 10.1016/j.bpj.2016.12.031.

A. Hartman, G. Johansson, and P. Albertsson. Partition of Proteins in a Three-Phase System. European Journal of Biochemistry, 46(1):75-81, 1974. ISSN 14321033. doi: 10.1111/j.1432-1033.1974.tb03598.x.

R. A. Heidemann. The criteria for thermodynamic stability. AlChE Journal, 21(4):824-826, 1975. ISSN 0001-1541. doi: 10.1002/aic.690210433. 
R. A. Heidemann. The Classical Theory of Critical Points. In Supercritical Fluids, pages 39-64. Springer Netherlands, 1994. doi: 10.1007/978-94-015-8295-7_2.

R. A. Heidemann and A. M. Khalil. The calculation of critical points. A/ChE Journal, 26 (5):769-779, 1980. ISSN 15475905. doi: 10.1002/aic.690260510.

T. L. Hill. An Introduction to Statistical Thermodynamics. Dover Publications, New York, 1986. ISBN 9780486652429.

S. Ji and J. Y. Walz. Interaction potentials between two colloidal particles surrounded by an extremely bidisperse particle suspension. Journal of Colloid and Interface Science, 394 (1):611-618, 2013. ISSN 00219797. doi: 10.1016/j.jcis.2012.11.040.

G. Johansson and H. Walter. Partitioning and concentrating biomaterials in aqueous phase systems. International Review of Cytology, 192(1896):33-60, 1999. ISSN 00747696. doi: 10.1016/s0074-7696(08)60521-5.

H. N. Lekkerkerker and R. Tuinier. Colloids and the Depletion Interaction, volume 833. Springer, 2011. ISBN 978-94-007-1222-5. doi: 10.1007/978-94-007-1223-2.

C. R. Mace, O. Akbulut, A. A. Kumar, N. D. Shapiro, R. Derda, M. R. Patton, and G. M. Whitesides. Aqueous Multiphase Systems of Polymers and Surfactants Provide Self-Assembling Step-Gradients in Density. Journal of the American Chemical Society, 134(22):9094-9097, 2012. ISSN 0002-7863. doi: 10.1021/ja303183z.

N. Park and J. C. Conrad. Phase behavior of colloid-polymer depletion mixtures with unary or binary depletants. Soft Matter, 13(15):2781-2792, 2017. ISSN 17446848. doi: 10.1039/C6SM02891H.

R. C. Reid and B. L. Beegle. Critical point criteria in legendre transform notation. AlChE Journal, 23(5):726-732, 1977. ISSN 15475905. doi: 10.1002/aic.690230515.

R. Roth, R. Evans, and A. A. Louis. Theory of asymmetric nonadditive binary hard-sphere mixtures. Physical Review E - Statistical Physics, Plasmas, Fluids, and Related Interdisciplinary Topics, 64(5):13, 2001. ISSN 1063651X. doi: 10.1103/PhysRevE.64.051202.

K. Ruan, B. H. Wang, J. X. Xiao, and J. N. Tang. Interfacial tension of aqueous threephase systems formed by Triton X-100/PEG/Dextran. Journal of Dispersion Science and Technology, 27(7):927-930, 2006. ISSN 01932691. doi: 10.1080/01932690600766223.

R. P. Sear and J. a. Cuesta. Instabilities in complex mixtures with a large number of components. Physical Review Letters, 91(24):245701, 2003. ISSN 10797114. doi: 10.1103/PhysRevLett.91.245701. 
M. A. Solokhin, A. V. Solokhin, and V. S. Timofeev. Phase-equilibrium stability criterion in terms of the eigenvalues of the Hessian matrix of the Gibbs potential. Theoretical Foundations of Chemical Engineering, 36(5):444-446, 2002. ISSN 00405795. doi: 10.1023/A:1020617610644.

L. Sturtewagen and E. van der Linden. Effect of polydispersity on the phase behavior of additive hard spheres in solution, part I. Manuscript submitted, 2019. URL http://arxiv.org/abs/1912.03143.

Z. Zhao, Q. Li, X. Ji, R. Dimova, R. Lipowsky, and Y. Liu. Molar mass fractionation in aqueous two-phase polymer solutions of dextran and poly(ethylene glycol). Journal of Chromatography A, 1452:107-115, 2016. ISSN 18733778. doi: 10.1016/j.chroma.2016.04.075. 


\section{A Tables}

Table 4.A.1: Fractionation of monodisperse ternary (component $A, B$, and $C$ ) non-additive hard sphere mixtures with size ratio $q_{A B}=\sigma_{A} / \sigma_{B}=1 / 4$ and $q_{A C}=\sigma_{A} / \sigma_{C}=1 / 3$, with non-additivity parameters: $\Delta_{A B}=0.1, \Delta_{A C}$ and $\Delta_{B C}$ variying from -0.1 to 0.1 , label refering to Figures 4.3 to 4.11, fixed parent phase: $\eta(0.05,0.20,0.10)$, see also Figure 4.12a.

\begin{tabular}{|c|c|c|}
\hline Mixture & Top phase & Bottom phase \\
\hline 4.3 & $\begin{array}{l}\eta(0.061,0.054,0.104) \\
\alpha: 0.733\end{array}$ & $\begin{array}{l}\eta(0.021,0.602,0.089) \\
\alpha: 0.267\end{array}$ \\
\hline 4.4 & $\begin{array}{l}\eta(0.060,0.046,0.117) \\
\alpha: 0.770\end{array}$ & $\begin{array}{l}\eta(0.017,0.716,0.043) \\
\alpha: 0.223\end{array}$ \\
\hline 4.5 & $\begin{array}{l}\eta(0.063,0.044,0.087) \\
\alpha: 0.682\end{array}$ & $\begin{array}{l}\eta(0.022,0.534,0.127) \\
\alpha: 0.318\end{array}$ \\
\hline 4.6 & $\begin{array}{l}\eta(0.060,0.055,0.107) \\
\alpha: 0.749\end{array}$ & $\begin{array}{l}\eta(0.020,0.633,0.080) \\
\alpha: 0.251\end{array}$ \\
\hline 4.7 & $\begin{array}{l}\eta(0.069,0.020,0.049) \\
\alpha: 0.611\end{array}$ & $\begin{array}{l}\eta(0.020,0.483,0.180) \\
\alpha: 0.389\end{array}$ \\
\hline 4.8 & $\begin{array}{l}\eta(0.066,0.034,0.073) \\
\alpha: 0.622\end{array}$ & $\begin{array}{l}\eta(0.024,0.473,0.145) \\
\alpha: 0.378\end{array}$ \\
\hline 4.9 & $\begin{array}{l}\eta(0.060,0.027,0.126) \\
\alpha: 0.779\end{array}$ & $\begin{array}{l}\eta(0.014,0.808,0.010) \\
\alpha: 0.222\end{array}$ \\
\hline 4.10 & $\begin{array}{l}\eta(0.059,0.040,0.121) \\
\alpha: 0.790\end{array}$ & $\begin{array}{l}\eta(0.015,0.803,0.020) \\
\alpha: 0.210\end{array}$ \\
\hline 4.11 & $\begin{array}{l}\eta(0.059,0.057,0.113) \\
\alpha: 0.787\end{array}$ & $\begin{array}{l}\eta(0.017,0.731,0.052) \\
\alpha: 0.213\end{array}$ \\
\hline
\end{tabular}


A. Tables

Table 4.A.2: Fractionation of monodisperse ternary (component $A, B$, and $C$ ) non-additive hard sphere mixtures with size ratio $q_{A B}=\sigma_{A} / \sigma_{B}=1 / 4$ and $q_{A C}=\sigma_{A} / \sigma_{C}=1 / 3$, with non-additivity parameters: $\Delta_{A B}=0.1, \Delta_{A C}$ and $\Delta_{B C}$ variying from -0.1 to 0.1 , label refering to Figures 4.3 to $\mathbf{4 . 1 1}$, fixed parent phase: $\eta(0.05,0.20,0.25)$, see also Figure $\mathbf{4 . 1 2 b}$.

\begin{tabular}{|c|c|c|}
\hline Mixture & Top phase & Bottom phase \\
\hline 4.3 & $\begin{array}{l}\eta(0.060,0.054,0.257) \\
\alpha: 0.742\end{array}$ & $\begin{array}{l}\eta(0.020,0.619,0.230) \\
\alpha: 0.258\end{array}$ \\
\hline 4.4 & $\begin{array}{l}\eta(0.059,0.040,0.284) \\
\alpha: 0.813\end{array}$ & $\begin{array}{l}\eta(0.013,0.894,0.105) \\
\alpha: 0.187\end{array}$ \\
\hline 4.5 & $\begin{array}{l}\eta(0.065,0.037,0.223) \\
\alpha: 0.644\end{array}$ & $\begin{array}{l}\eta(0.023,0.495,0.298) \\
\alpha: 0.356\end{array}$ \\
\hline 4.6 & $\begin{array}{l}\eta(0.059,0.057,0.265) \\
\alpha: 0.788\end{array}$ & $\begin{array}{l}\eta(0.018,0.715,0.195) \\
\alpha: 0.217\end{array}$ \\
\hline 4.7 & $\begin{array}{l}\eta(0.084,0.003,0.086) \\
\alpha: 0.468\end{array}$ & $\begin{array}{l}\eta(0.020,0.373,0.394) \\
\alpha: 0.532\end{array}$ \\
\hline 4.8 & $\begin{array}{l}\eta(0.085,0.007,0.103) \\
\alpha: 0.406\end{array}$ & $\begin{array}{l}\eta(0.026,0.332,0.350) \\
\alpha: 0.594\end{array}$ \\
\hline 4.9 & $\begin{array}{l}\eta(0.059,0.012,0.300) \\
\alpha: 0.827\end{array}$ & $\begin{array}{l}\eta(0.009,1.097,0.013) \\
\alpha: 0.173\end{array}$ \\
\hline 4.10 & $\begin{array}{l}\eta(0.058,0.023,0.294) \\
\alpha: 0.838\end{array}$ & $\begin{array}{l}\eta(0.009,1.116,0.023) \\
\alpha: 0.162\end{array}$ \\
\hline 4.11 & $\begin{array}{l}\eta(0.057,0.048,0.284) \\
\alpha: 0.855\end{array}$ & $\begin{array}{l}\eta(0.011,1.095,0.050) \\
\alpha: 0.145\end{array}$ \\
\hline
\end{tabular}


Table 4.A.3: Fractionation of monodisperse ternary (component $A, B$, and $C$ ) non-additive hard sphere mixtures with size ratio $q_{A B}=\sigma_{A} / \sigma_{B}=1 / 4$ and $q_{A C}=\sigma_{A} / \sigma_{C}=1 / 3$, with non-additivity parameters: $\Delta_{A B}=0.1, \Delta_{A C}$ and $\Delta_{B C}$ variying from -0.1 to 0.1 , label refering to Figures 4.3 to 4.11, fixed parent phase: $\eta(0.10,0.20,0.10)$, see also Figure 4.13a.

\begin{tabular}{|c|c|c|c|}
\hline Mixture & Top phase & Middle phase & Bottom phase \\
\hline 4.3 & $\begin{array}{l}\eta(0.116,0.001,0.102) \\
\alpha: 0.852\end{array}$ & & $\begin{array}{l}\eta(0.009,1.345,0.089) \\
\alpha: 0.148\end{array}$ \\
\hline 4.4 & $\begin{array}{l}\eta(0.115,0.001,0.113) \\
\alpha: 0.864\end{array}$ & & $\begin{array}{l}\eta(0.007,1.461,0.015) \\
\alpha: 0.136\end{array}$ \\
\hline 4.5 & $\begin{array}{l}\eta(0.119,0.001,0.070) \\
\alpha: 0.823\end{array}$ & & $\begin{array}{l}\eta(0.010,1.125,0.240) \\
\alpha: 0.177\end{array}$ \\
\hline 4.6 & $\begin{array}{l}\eta(0.116,0.001,0.104) \\
\alpha: 0.854\end{array}$ & & $\begin{array}{l}\eta(0.008,1.367,0.080) \\
\alpha: 0.146\end{array}$ \\
\hline 4.7 & $\begin{array}{l}\eta(0.126,0.000,0.013) \\
\alpha: 0.774\end{array}$ & & $\begin{array}{l}\eta(0.010,0.886,0.398) \\
\alpha: 0.226\end{array}$ \\
\hline 4.8 & $\begin{array}{l}\eta(0.127,0.000,0.026) \\
\alpha: 0.766\end{array}$ & & $\begin{array}{l}\eta(0.013,0.855,0.344) \\
\alpha: 0.234\end{array}$ \\
\hline 4.9 & $\begin{array}{l}\eta(0.114,0.001,0.115) \\
\alpha: 0.866\end{array}$ & & $\begin{array}{l}\eta(0.007,1.489,0.001) \\
\alpha: 0.134\end{array}$ \\
\hline 4.10 & $\begin{array}{l}\eta(0.114,0.001,0.114) \\
\alpha: 0.869\end{array}$ & & $\begin{array}{l}\eta(0.007,1.517,0.005) \\
\alpha: 0.131\end{array}$ \\
\hline 4.11 & $\begin{array}{l}\eta(0.123,0.001,0.051) \\
\alpha: 0.778\end{array}$ & $\begin{array}{l}\eta(0.035,0.044,0.636) \\
\alpha: 0.087\end{array}$ & $\begin{array}{l}\eta(0.008,1.451,0.036) \\
\alpha: 0.135\end{array}$ \\
\hline
\end{tabular}


A. Tables

Table 4.A.4: Fractionation of monodisperse ternary (component $A, B$, and $C$ ) non-additive hard sphere mixtures with size ratio $q_{A B}=\sigma_{A} / \sigma_{B}=1 / 4$ and $q_{A C}=\sigma_{A} / \sigma_{C}=1 / 3$, with non-additivity parameters: $\Delta_{A B}=0.1, \Delta_{A C}$ and $\Delta_{B C}$ variying from -0.1 to 0.1 , label refering to Figures 4.3 to $\mathbf{4 . 1 1}$, fixed parent phase: $\eta(0.05,0.10,0.10)$, see also Figure $\mathbf{4 . 1 3 b}$.

\begin{tabular}{lll}
\hline Mixture & Top phase & Bottom phase \\
\hline 4.3 & & $\begin{array}{l}\eta(0.050,0.100,0.100) \\
\alpha: 1\end{array}$ \\
\hline 4.4 & $\begin{array}{l}\eta(0.051,0.083,0.102) \\
\alpha: 0.964\end{array}$ & $\begin{array}{l}\eta(0.021,0.5610 .050) \\
\alpha: 0.036\end{array}$ \\
\hline 4.5 & & $\eta(0.050,0.100,0.100)$ \\
& & $\alpha: 1$ \\
\hline 4.6 & & $\eta(0.050,0.100,0.100)$ \\
& & $\alpha: 1$ \\
\hline 4.7 & $\eta(0.056,0.047,0.082)$ & $\begin{array}{l}\eta(0.027,0.315,0.172) \\
\alpha: 0.197\end{array}$ \\
\hline 4.8 & $\alpha: 0.803$ & $\eta(0.032,0.289,0.132)$ \\
& $\alpha: 0.956$ & $\alpha: 0.044$ \\
\hline 4.9 & $\eta(0.053,0.047,0.108)$ & $\eta(0.016,0.675,0.014)$ \\
& $\alpha: 0.915$ & $\alpha: 0.085$ \\
\hline 4.10 & $\eta(0.052,0.067,0.104)$ & $\eta(0.018,0.661,0.026)$ \\
& $\alpha: 0.944$ & $\alpha: 0.056$ \\
\hline 4.11 & $\eta(0.050,0.098,0.100)$ & $\begin{array}{l}\eta(0.021,0.573,0.057) \\
\alpha: 0.004\end{array}$ \\
\hline
\end{tabular}




\section{B Fractionation}
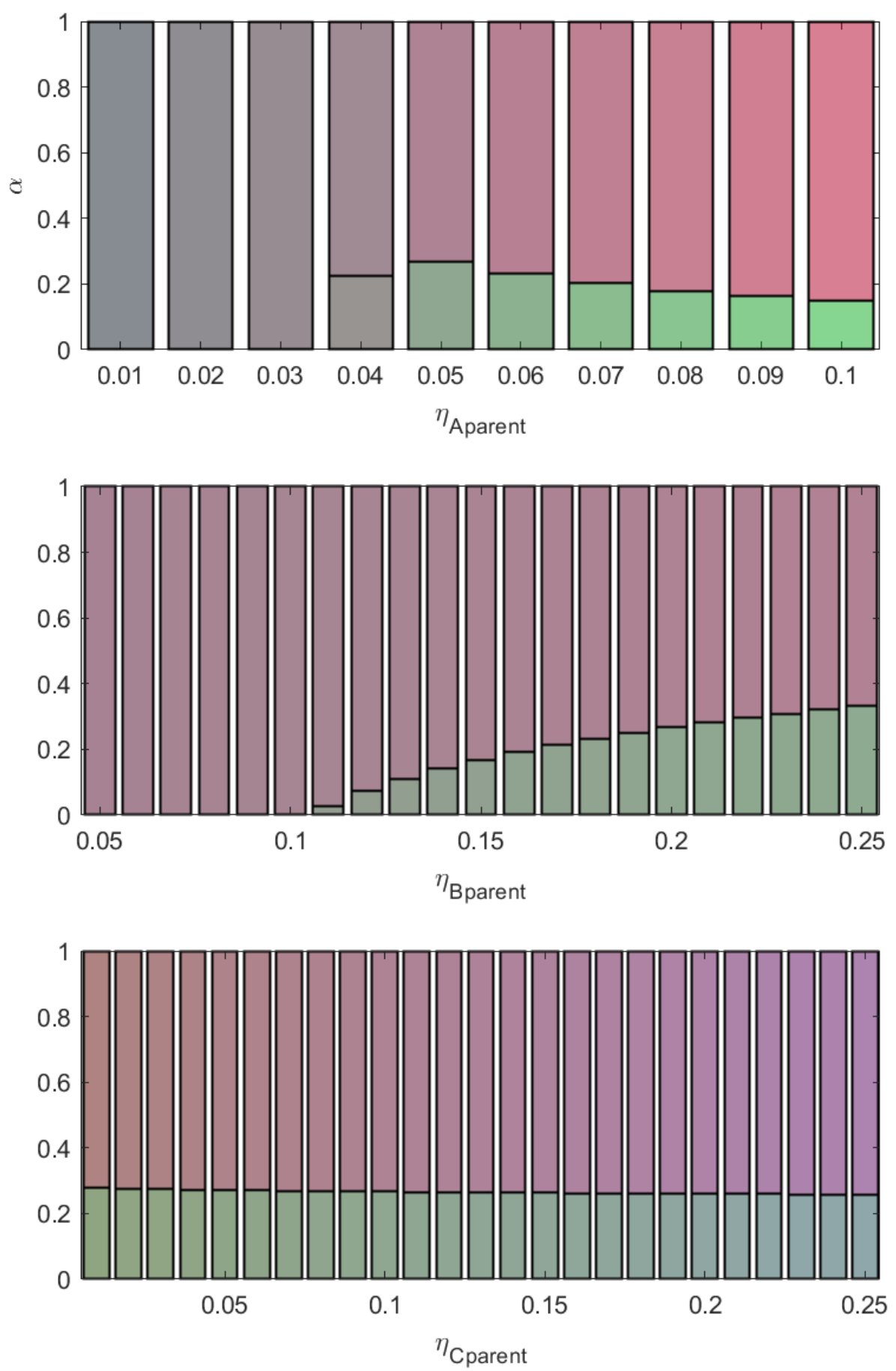

Figure 4.B.1: Fractionation of monodisperse ternary (component $A, B$, and $C$ ) non-additive hard sphere mixture with size ratio $q_{A B}=\sigma_{A} / \sigma_{B}=1 / 4$ and $q_{A C}=\sigma_{A} / \sigma_{C}=1 / 3$, with non-additivity parameters: $\Delta_{A B}=0.1, \Delta_{A C}=-0.1$, and $\Delta_{B C}=-0.1$, fixed parent phase: $\eta(0.05,0.20,0.10)$, adjusting $A, B$, resp. $C$ with $\eta=0.01, A$ is red $\square, B$ is green $\square$, and $C$ is blue $\square$. 

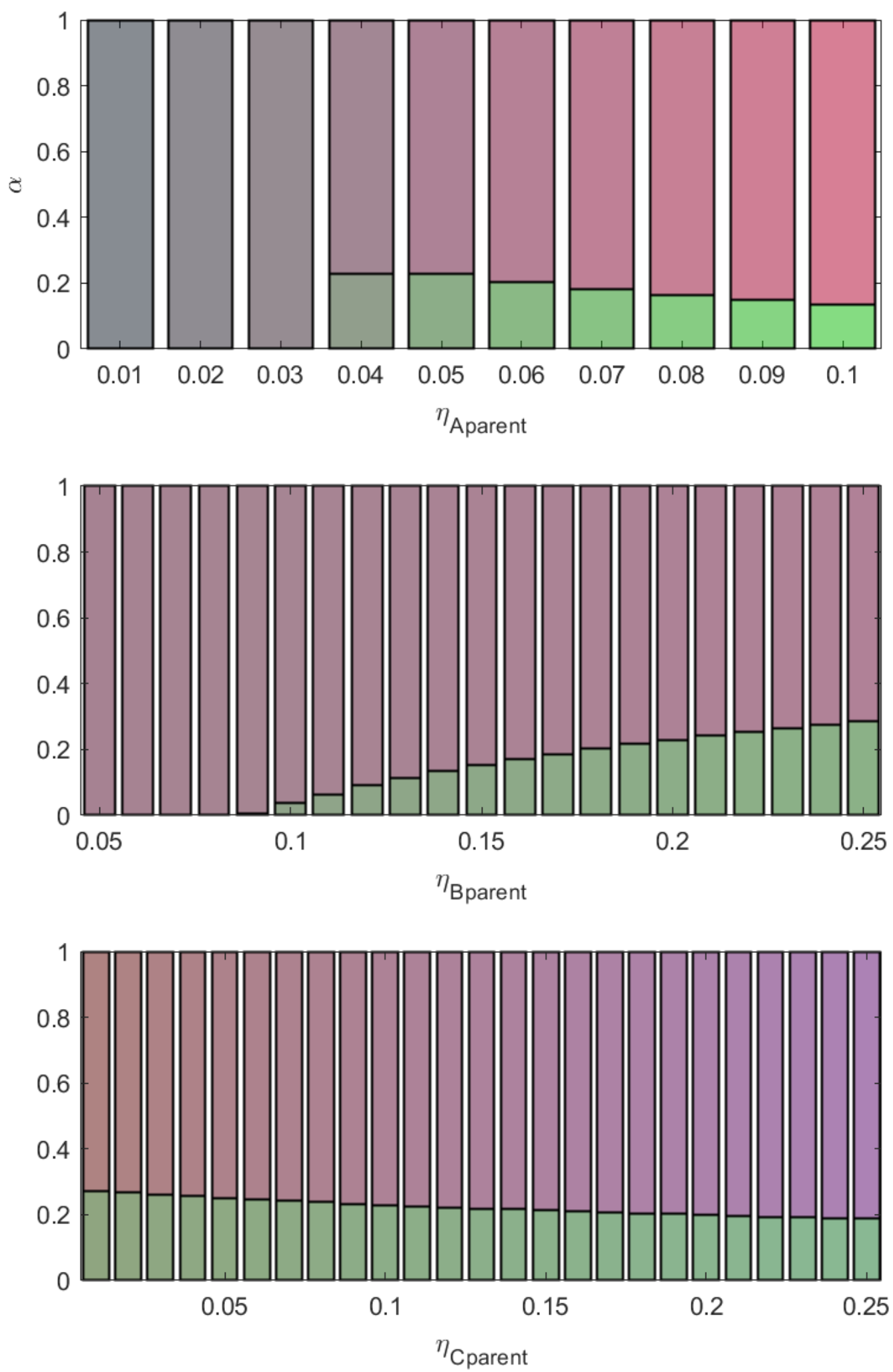

Figure 4.B.2: Fractionation of monodisperse ternary (component $A, B$, and $C$ ) non-additive hard sphere mixture with size ratio $q_{A B}=\sigma_{A} / \sigma_{B}=1 / 4$ and $q_{A C}=\sigma_{A} / \sigma_{C}=1 / 3$, with non-additivity parameters: $\Delta_{A B}=0.1, \Delta_{A C}=-0.1$, and $\Delta_{B C}=0$, fixed parent phase: $\eta(0.05,0.20,0.10)$, adjusting $A, B$, resp. $C$ with $\eta=0.01, A$ is red $\square, B$ is green $\square$, and $C$ is blue $\square$. 

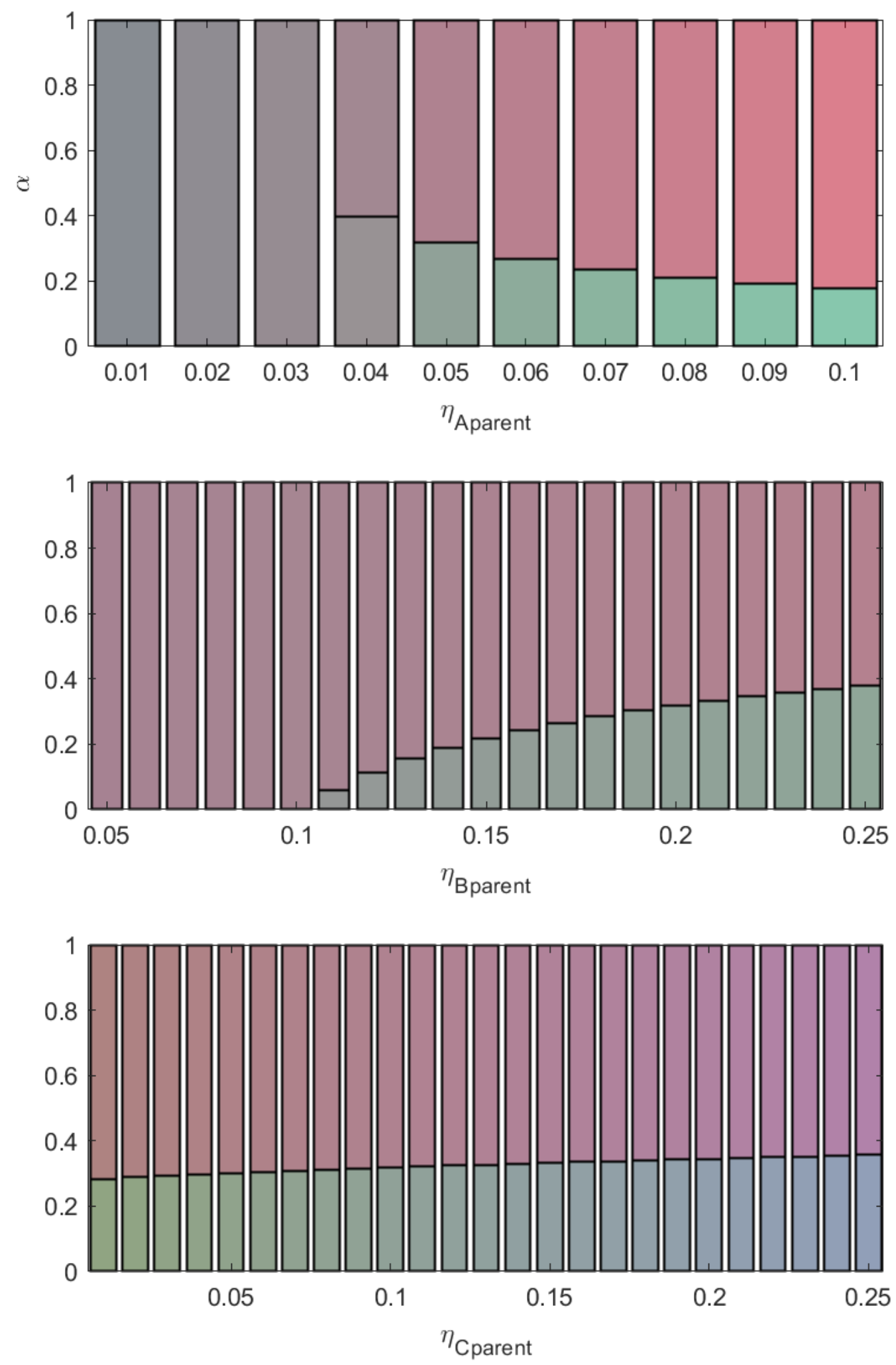

Figure 4.B.3: Fractionation of monodisperse ternary (component $A, B$, and $C$ ) non-additive hard sphere mixture with size ratio $q_{A B}=\sigma_{A} / \sigma_{B}=1 / 4$ and $q_{A C}=\sigma_{A} / \sigma_{C}=1 / 3$, with non-additivity parameters: $\Delta_{A B}=0.1, \Delta_{A C}=0$, and $\Delta_{B C}=-0.1$, fixed parent phase: $\eta(0.05,0.20,0.10)$, adjusting $A, B$, resp. $C$ with $\eta=0.01, A$ is red $\square, B$ is green $\square$, and $C$ is blue $\square$. 
B. Fractionation
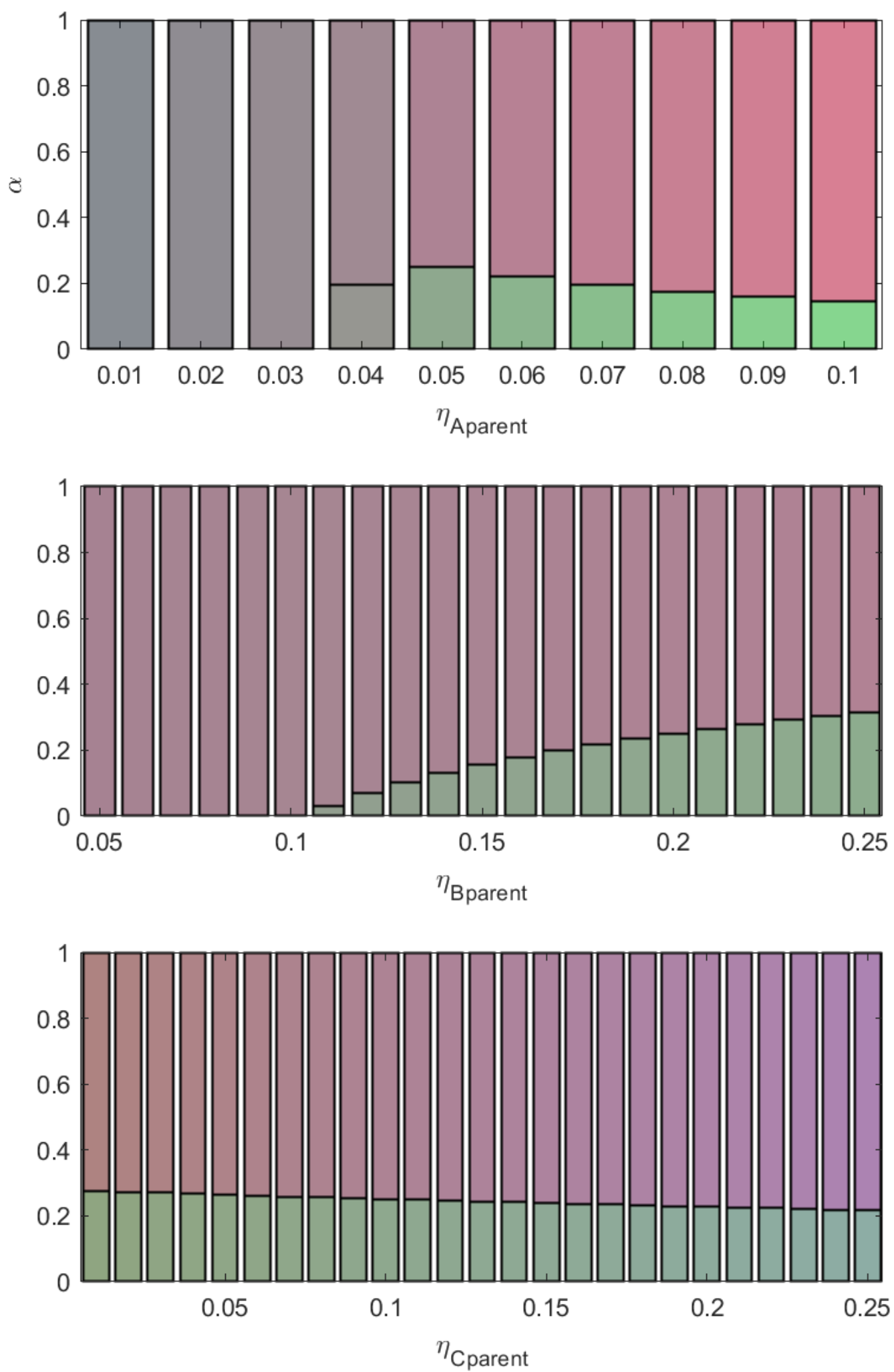

Figure 4.B.4: Fractionation of monodisperse ternary (component $A, B$, and $C$ ) non-additive hard sphere mixture with size ratio $q_{A B}=\sigma_{A} / \sigma_{B}=1 / 4$ and $q_{A C}=\sigma_{A} / \sigma_{C}=1 / 3$, with non-additivity parameters: $\Delta_{A B}=0.1, \Delta_{A C}=0$, and $\Delta_{B C}=0$, fixed parent phase: $\eta(0.05,0.20,0.10)$, adjusting $A, B$, resp. $C$ with $\eta=0.01, A$ is red $\square, B$ is green $\square$, and $C$ is blue $\square$. 

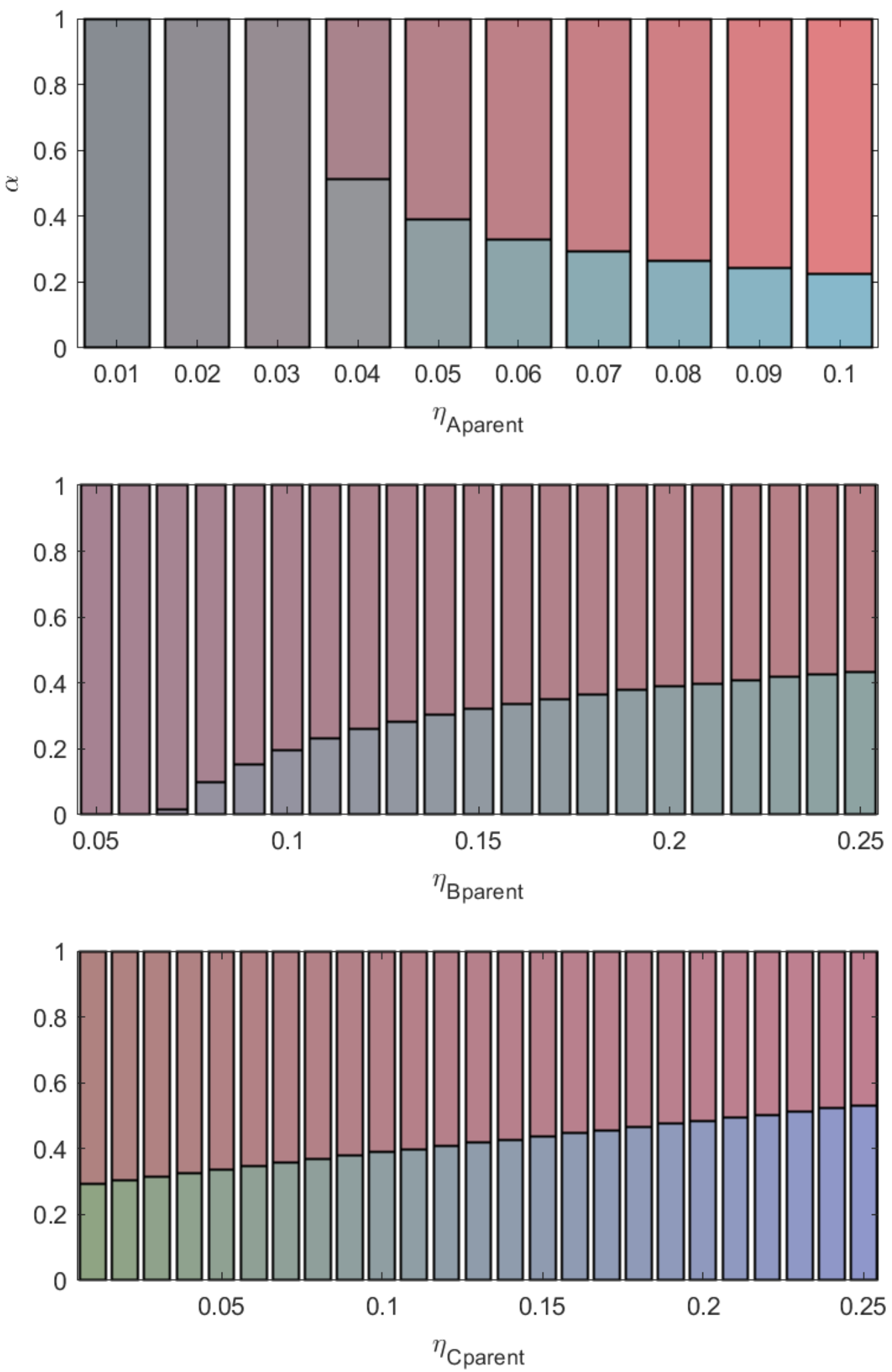

Figure 4.B.5: Fractionation of monodisperse ternary (component $A, B$, and $C$ ) non-additive hard sphere mixture with size ratio $q_{A B}=\sigma_{A} / \sigma_{B}=1 / 4$ and $q_{A C}=\sigma_{A} / \sigma_{C}=1 / 3$, with non-additivity parameters: $\Delta_{A B}=0.1, \Delta_{A C}=0.1$, and $\Delta_{B C}=-0.1$, fixed parent phase: $\eta(0.05,0.20,0.10)$, adjusting $A, B$, resp. $C$ with $\eta=0.01, A$ is red $\square, B$ is green $\square$, and $C$ is blue $\square$. 
B. Fractionation
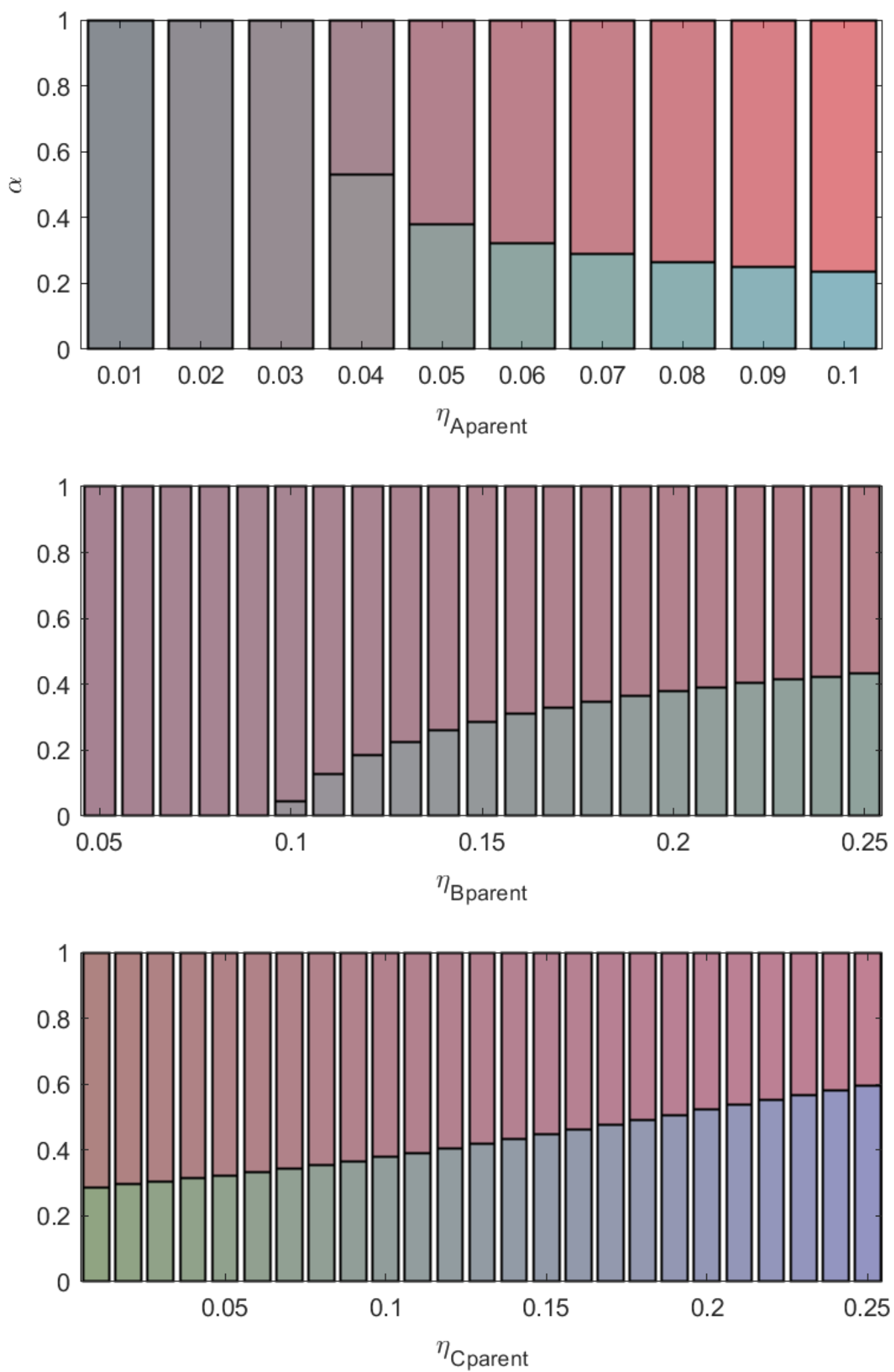

Figure 4.B.6: Fractionation of monodisperse ternary (component $A, B$, and $C$ ) non-additive hard sphere mixture with size ratio $q_{A B}=\sigma_{A} / \sigma_{B}=1 / 4$ and $q_{A C}=\sigma_{A} / \sigma_{C}=1 / 3$, with non-additivity parameters: $\Delta_{A B}=0.1, \Delta_{A C}=0.1$, and $\Delta_{B C}=0$, fixed parent phase: $\eta(0.05,0.20,0.10)$, adjusting $A, B$, resp. $C$ with $\eta=0.01, A$ is red $\square, B$ is green $\square$, and $C$ is blue $\square$. 

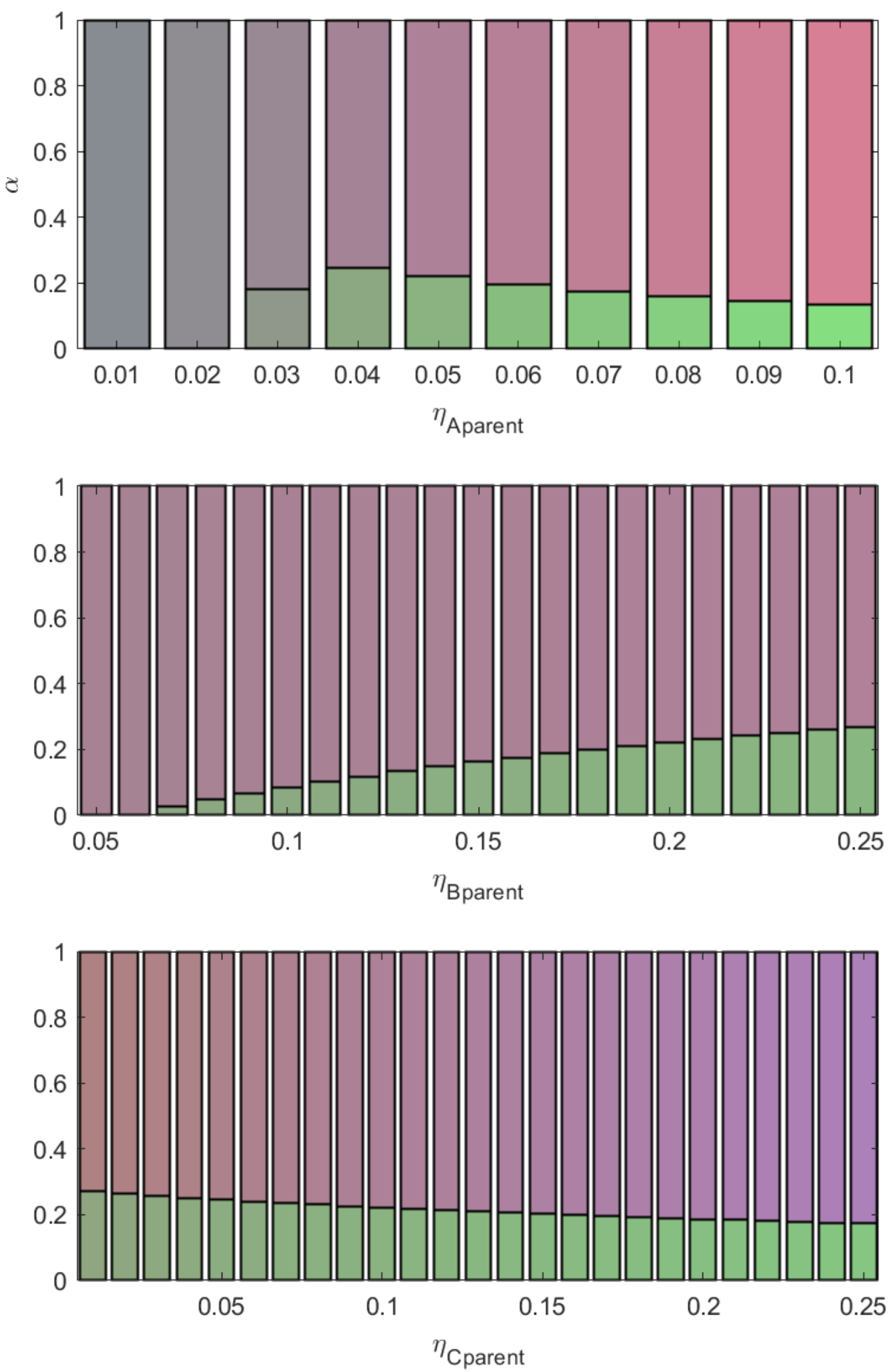

Figure 4.B.7: Fractionation of monodisperse ternary (component $A, B$, and $C$ ) non-additive hard sphere mixture with size ratio $q_{A B}=\sigma_{A} / \sigma_{B}=1 / 4$ and $q_{A C}=\sigma_{A} / \sigma_{C}=1 / 3$, with non-additivity parameters: $\Delta_{A B}=0.1, \Delta_{A C}=-0.1$, and $\Delta_{B C}=0.1$, fixed parent phase: $\eta(0.05,0.20,0.10)$, adjusting $A, B$, resp. $C$ with $\eta=0.01, A$ is red $\square, B$ is green $\square$, and $C$ is blue $\square$. 
B. Fractionation
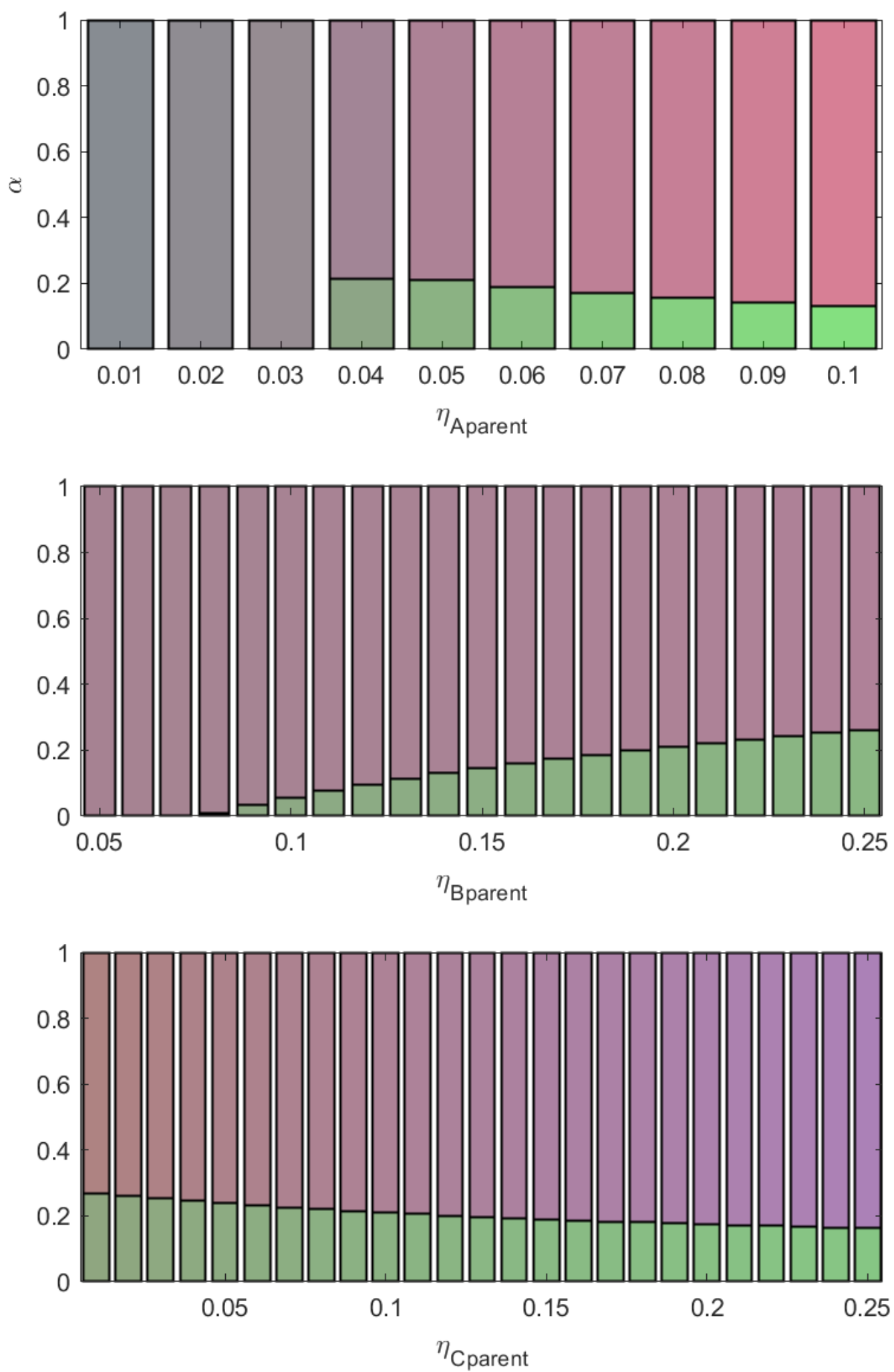

Figure 4.B.8: Fractionation of monodisperse ternary (component $A, B$, and $C$ ) non-additive hard sphere mixture with size ratio $q_{A B}=\sigma_{A} / \sigma_{B}=1 / 4$ and $q_{A C}=\sigma_{A} / \sigma_{C}=1 / 3$, with non-additivity parameters: $\Delta_{A B}=0.1, \Delta_{A C}=0$, and $\Delta_{B C}=0.1$, fixed parent phase: $\eta(0.05,0.20,0.10)$, adjusting $A, B$, resp. $C$ with $\eta=0.01, A$ is red $\square, B$ is green $\square$, and $C$ is blue $\square$. 

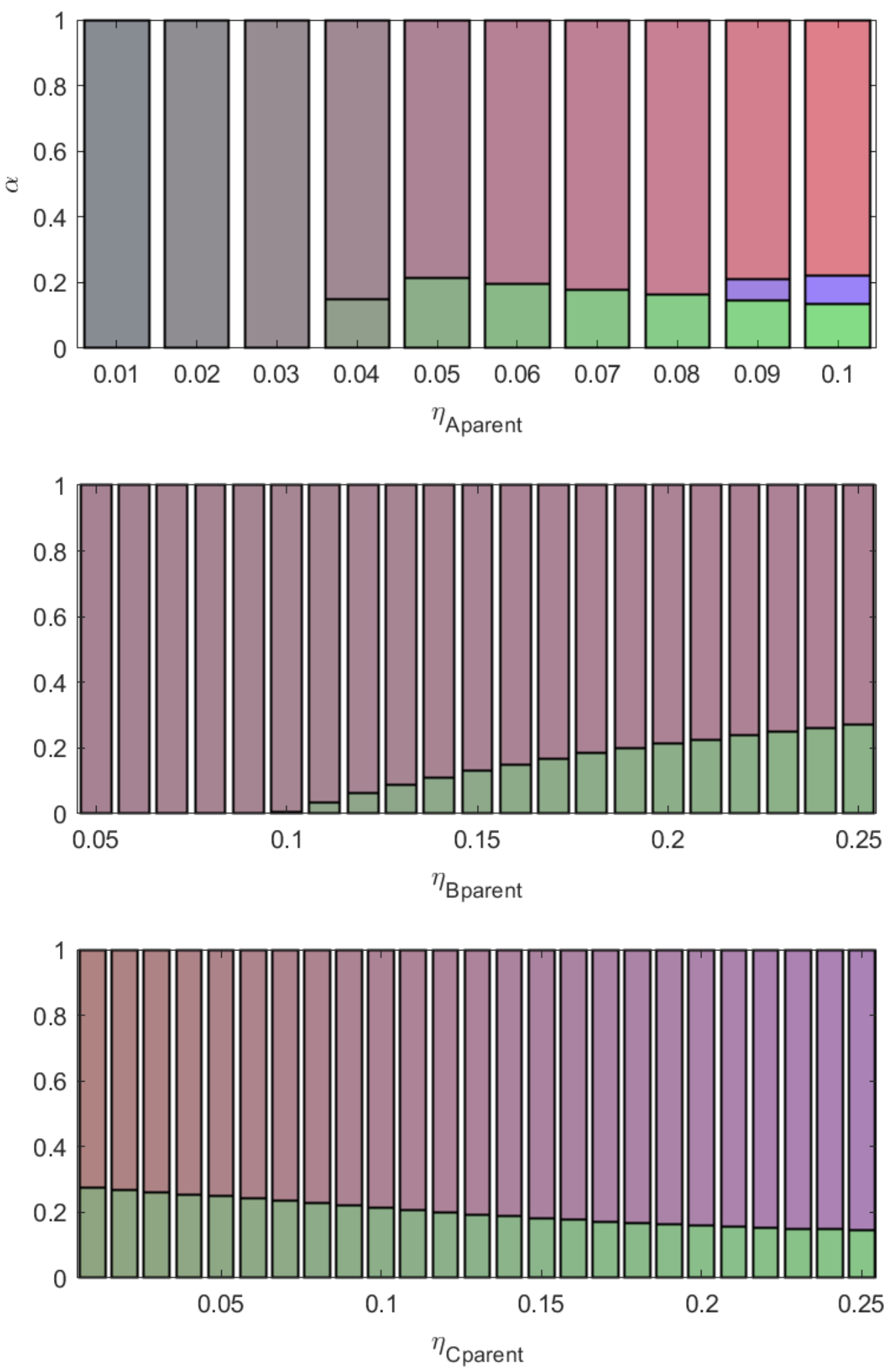

Figure 4.B.9: Fractionation of monodisperse ternary (component $A, B$, and $C$ ) non-additive hard sphere mixture with size ratio $q_{A B}=\sigma_{A} / \sigma_{B}=1 / 4$ and $q_{A C}=\sigma_{A} / \sigma_{C}=1 / 3$, with non-additivity parameters: $\Delta_{A B}=0.1, \Delta_{A C}=0.1$, and $\Delta_{B C}=0.1$, fixed parent phase: $\eta(0.05,0.20,0.10)$, adjusting $A, B$, resp. $C$ with $\eta=0.01, A$ is red $\square, B$ is green $\square$, and $C$ is blue $\square$. 


\section{Improving predictions of phase behavior of binary mixtures by taking polydispersity into account}

This chapter is based on: Luka Sturtewagen, Belinda P.C. Dewi, Paul Venema and Erik van der Linden. Improving predictions of phase behavior of binary mixtures by taking polydispersity into account. (to be submitted) 


\begin{abstract}
We study the phase behavior of two polydisperse hydrocolloids, dextran and polyethylene oxide. We combine the experimental osmometric virial coefficients of the pure components with the experimental critical point of their aqueous mixture and the size distribution of each component from a previously published study by Dewi et al. (2020) in order to predict the phase boundary, spinodal and fractionation upon demixing of the polydisperse mixture. We compare our results to the experimental phase diagram. This method shows a higher accuracy in predicting the binary phase behavior combined to modeling each component as monodisperse. The polydispersity of the hydrocolloids causes the phase separation boundary to shift to lower concentrations and the miscibility region to decrease. The shape of the phase boundary shifts from a rotated U-shape to a W-shape.
\end{abstract}




\subsection{Introduction}

Aqueous mixtures of polymers such as polyethylene oxide (PEO) and dextran form liquidliquid two phase systems at certain concentrations. These systems are often used as a model system for the phase behavior of macromolecules in solution (Kang and Sandler, 1988; Edelman et al., 2003; Dewi et al., 2020). They also have practical applications as they are often used to aid in the partitioning of biological materials such as proteins and cell materials (Johansson and Walter, 1999; Johansson et al., 1998).

For the prediction of their phase behavior, the polymers are often considered monodisperse. Experimental work by Edelman et al. (2003), however, has shown that there is considerable fractionation for both PEO and dextran upon demixing. Not only is there an effect of the polydispersity of each component on the fractionation, also the concentration in the parent phase plays a role. At lower total polymer concentrations in the parent phase, the depleted colloids have a broader distribution, i.e. there is less fractionation. This results in molar mass averages of both components in their depleted phases increasing and approximating the molar mass of the parent polymers, when the initial polymer concentration is decreased. Changes in the distribution are mainly prevalent in the depleted phase, as the average molar mass in the enriched phase does not change considerably depending on the parent concentration. Changes in the amount of fractionation depending on the concentration along a dilution line where also reported by Zhao et al. (2016). Also Gaube et al. (1993) showed that the polydispersity plays a role in the phase behavior and phase composition. They compared mixtures of PEO and dextran with various molecular sizes and found that for each polymer the short chain molecules preferentially partition to the phase enriched in the other polymer.

There has been some effort on incorporating the polydispersity of these polymers in the prediction of their phase behavior. Kang and Sandler (1988) incorporated the polydispersity in their prediction by using a universal quasi-chemical (UNIQUAC) model and found that the polydispersity of the polymers enlarged the two-phase region considerably near the critical point and resulted in smaller miscibility regions far from the critical point. They also found significant fractionation and the difference in average molecular weight of the components in each phase increased with larger polydispersity.

Most often when the phase behavior of binary mixture is studied experimentally, one or more dilution lines are used to obtain the concentration of each component in each phase. These concentrations are then used to form a binodal (Albertsson, 1970). This approach allows for finding the position of the binodal. However, this approach does not shed light on the polydispersity, nor does it give insight into the demixing in the metastable region, a concentration that demixes into multiple phases, but that is outside of the unstable 
spinodal region. It is also hard to quantify the impact of the polydispersity and the according distribution on the phase behavior (Dos Santos Tada et al., 2004). In order to get more insight into the transition between one and two phases, Larsson and Mattiasson (1988) determined the experimental phase boundary for polydisperse PEO and dextran. They found a significant broadening of the phase boundary compared to the approach of Albertsson (1970). The broadening increased with increasing polydispersity at the depleted side of said polydisperse component (Larsson and Mattiasson, 1992).

In our previous work (Sturtewagen and van der Linden, 2019a,b) we predicted the phase behavior of a polydisperse binary mixture of hard spheres in solution using the virial coefficient approach, based on the theory of McMillan and Mayer (1945). Polydispersity was incorporated into the system by means of sub-components. The spheres had an asymmetric size ratio to aid in demixing (Dijkstra et al., 1999). Polydispersity of the largest component caused significant changes to the phase diagram. With increased polydispersity, the critical point shifted to higher concentrations while also at the same time the miscible region decreased. Not only the polydispersity played a role, also the type of distribution had an influence. We found that the largest components of the distribution impacted the phase diagram the most. Demixed mixtures showed also significant fractionation. The smallest sub-components of the large polydisperse components favored to top phase enriched in the small monodisperse component.

In recent work the phase diagram for the macromolecules PEO and dextran was determined using the osmotic virial coefficients and the experimental critical point of the binary mixture (Dewi et al., 2020). The method allowed for predicting the phase behavior of the mixture. However, some points of the experimental phase diagram remained unexplained by the theory. Mainly at low concentrations of dextran the experimental system showed thermodynamic incompatibility and demixed into two phases, while the theory predicted a homogeneous mixture for these concentrations. We hypothesize that this discrepancy is due to the polydispersity of the macromolecules. In this work we will take the polydispersity of both these components into account (by sub-dividing each polymer into sub-components of different size) when predicting the phase diagram based on the experimental data from Dewi et al. (2020). We will study the position of the phase separation boundary, the spinodal, the critical point, and the fractionation. We model the interactions between the different polydisperse sub-components using the second virial coefficient (Section 5.2.1). In Section 5.2.2 we describe the equations for the stability boundary, the spinodal, in Section 5.2.3 we describe the equations for the critical point and finally in Section 5.2.4 we describe the equations defining the phase boundary. In Section 5.3 we explain how we fitted the virial coefficients of the polydisperse sub-components to the experimental data. With the expressions in Section 5.2 and the fitted virial coefficients we have enough to 
calculate the full phase diagram Section 5.4. In this section, we treat the experimental polydispersity of PEO (Section 5.4.1) and dextran (Section 5.4.1) separately before analyzing their combined effects in Section 5.4.3.

\subsection{Theoretical expressions}

We show the equations used for the calculations of the phase diagram for polydisperse binary mixtures: the set of equations defining the stability boundary, the critical point, and phase boundaries of a mixture. All sets of equations are solved in Matlab R2017b. For a more detailed derivation of the equations, we refer to Sturtewagen and van der Linden (2019a). The virial coefficients used to calculate the phase diagram are obtained from fitting experimental data from earlier published work (Dewi et al., 2020).

\subsubsection{Osmotic virial coefficient}

The osmotic pressure, $\Pi$, of a solution at a temperature $T$, can be written as a virial expansion, similar to the virial expansion of the universal gas law for real gasses (Hill, 1986):

$$
\beta \Pi=\rho+B_{2}\left(T, \mu_{s}\right) \rho^{2}+B_{3}\left(T, \mu_{s}\right) \rho^{3}+\ldots
$$

with $\beta=\frac{1}{k T}, k$ the Boltzmann's constant, $\rho$ the number density of the component $\left(\frac{N_{\nu}}{V}\right), B_{2}$ the second virial coefficient, and $B_{3}$ the third virial coefficient. The second virial coefficient accounts for the increase in osmotic pressure due to particle pairwise interaction. The third virial coefficient accounts for the interaction between three particles in a variety of configurations. The equation can be expanded for higher densities with $B_{n}$, the $n^{\text {th }}$ virial coefficient, which accounts for the interaction between $n$ different particles.

In this work we will limit the virial expansion to the second virial coefficient, which is given by (Lekkerkerker and Tuinier, 2011):

$$
B\left(T, \mu_{s}\right)=2 \pi \int_{0}^{\infty} r^{2}\left(1-\exp \left[-\beta W\left(r, \mu_{s}\right)\right]\right) d r
$$

in which $\mu_{s}$ is the chemical potential of the solution, $W(r)$ is the interaction potential between the particles, and $r$ is the distance.

For additive hard sphere (HS) interaction, the interaction potential for two particles (of the same species or different species) is given by:

$$
W(r)_{H S}= \begin{cases}0, & r>\sigma_{i j} \\ \infty, & r \leq \sigma_{i j}\end{cases}
$$


with $\sigma_{i j}=\left(\sigma_{i}+\sigma_{j}\right) / 2$, the distance between the centers of the two particles, and $\sigma_{i}$ and $\sigma_{j}$ their respective diameter.

For non-additive hard spheres (NAHS), the distance of the closest approach of the centers of the two particles of different species can be closer or further than the distance between their centers (Roth et al., 2001). The closest distance then becomes: $\sigma_{i j}=\left(\left(\sigma_{i}+\sigma_{j}\right) / 2\right)(1+\Delta)$, in which $\Delta(\geq-1)$ accounts for the non-additivity of the interaction between the particles. When $\Delta>0$ the distance of closest approach of both spheres increases and when $\Delta<0$ the distance of closest approach decreases compared to that due to HS interaction only. For additive hard sphere interaction $\Delta=0$.

In a mixture with $n$ distinguishable components in a solution, there are two main types of two particle interactions that can occur: between particles of the same species and particles of different species.

For the second virial coefficient given by Eq. (5.2), using the interaction potential defined in Eq. (5.3), we find:

$$
\begin{aligned}
& B_{x x}=\frac{2 \pi}{3}\left(\sigma_{x}\right)^{3} \\
& B_{x y}=\frac{2 \pi}{3}\left(\left(\frac{\sigma_{x}+\sigma_{y}}{2}\right)(1+\Delta)\right)^{3}
\end{aligned}
$$

where $B_{x x}$ is the second virial coefficient for particles of the same species (assumed to be HS) and $B_{x y}$ is the second virial coefficient of particles of different species, which can be HS or NAHS.

The general equation for the osmotic pressure for a dilute binary mixture of polydisperse components, as represented in Figure 5.1, limited to the second virial coefficient, is given by (Sturtewagen and van der Linden, 2019a):

$$
\begin{aligned}
\beta \Pi=\rho_{A_{1}} & +\ldots+\rho_{A_{N_{A}}}+\rho_{B_{1}}+\ldots+\rho_{B_{N_{B}}} \\
& +B_{A_{1} A_{1}} \rho_{A_{1}}^{2}+2 B_{A_{1} A_{2}} \rho_{A_{1}} \rho_{A_{2}}+\ldots+B_{A_{N_{A}} A_{N_{A}} \rho_{A_{N_{A}}}^{2}} \\
& +2 B_{A_{1} B_{1}} \rho_{A_{1}} \rho_{B_{1}}+2 B_{A_{1} B_{2}} \rho_{A_{1}} \rho_{B_{2}}+\ldots+2 B_{A_{N_{A}} B_{N_{B}}} \rho_{B_{N_{B}}} \rho_{B_{N_{B}}} \\
& +B_{B_{1} B_{1}} \rho_{B_{1}}^{2}+2 B_{B_{1} B_{2}} \rho_{B_{1}} \rho_{B_{2}}+\ldots+B_{B_{N_{B}} B_{N_{B}}} \rho_{B_{N_{B}}}^{2}
\end{aligned}
$$

The number averaged second virial coefficient of a polydisperse component can be written as, this corresponds to the osmometric virial coefficient as measured by an osmometer Ersch et al. (2016):

$$
\begin{aligned}
B_{p d} & =B_{11} x_{1}^{2}+2 B_{12} x_{1} x_{2}+2 B_{13} x_{1} x_{3} \ldots \\
& =\sum_{i}^{m} \sum_{j}^{m} B_{i j} x_{i} x_{j}
\end{aligned}
$$




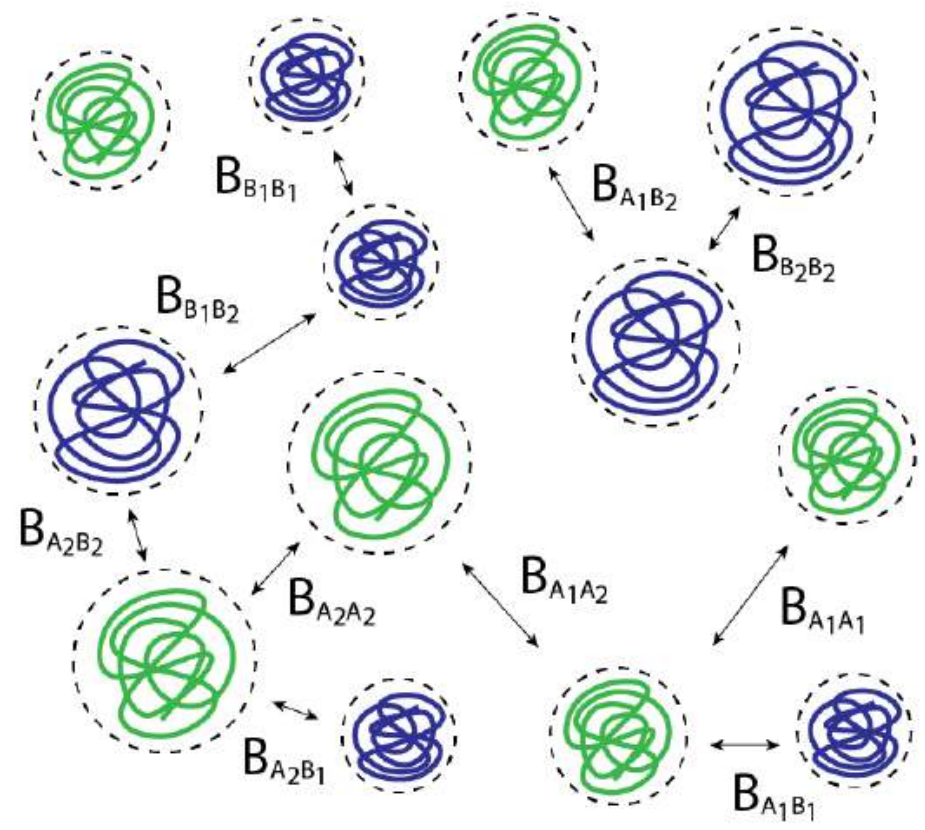

Figure 5.1: Graphical representation of a mixture of colloids, second virial coefficients are indicated. Colloid $A$ is indicated in green, colloid $B$ is indicated in blue.

in which $B_{i i}$ is the second virial coefficient of the component of the $i^{\text {th }}$ size, $B_{i j}$ is the second cross virial coefficient of particle the $i^{\text {th }}$ size and the particle of the $j^{\text {th }}$ size, and $x_{i}$ is the fraction of the particle of the $i^{\text {th }}$ size, $\sum x_{i}=1$.

This results in the following simplified equation for a binary mixture of polydisperse components:

$$
\beta \Pi=\rho_{A}+\rho_{B}+B_{A p d} \rho_{A}^{2}+2 B_{A B p d} \rho_{A} \rho_{B}+B_{B p d} \rho_{B}^{2}
$$

\subsubsection{Stability of a mixture}

The stability of a mixture is dependent on the second derivative of the free energy. If the second derivative of the mixture becomes zero, the mixture is at the boundary of becoming unstable. Unstable mixtures have a negative second derivative (Heidemann, 1975; Beegle et al., 1974).

The differential of the free energy of a mixture is given by (Hill, 1986):

$$
d A=-S d T-p d V+\sum_{i}^{n} \mu_{i} d N_{i}
$$

in which $\mu_{i}$, the chemical potential (the first partial derivative of the free energy with respect to number of particles $\left.\left(N_{i}\right)\right)$ for component $i$ is given by: 


$$
\mu_{i}=\mu_{i}^{0}+k T \ln \left(\rho_{i}\right)+2 k T\left(\sum_{j}^{n} B_{i j} \rho_{j}\right)
$$

For a mixture with $n$ distinguishable components, the second partial derivatives can be represented by a $n \times n$ matrix of the first partial derivatives of the chemical potential of each component.

This results in the following general stability matrix:

$$
\begin{aligned}
M_{1}= & {\left[\begin{array}{ccc}
\frac{\partial \mu_{1}}{\partial N_{1}} & \cdots & \frac{\partial \mu_{1}}{\partial N_{n}} \\
\vdots & \ddots & \vdots \\
\frac{\partial \mu_{n}}{\partial N_{1}} & \cdots & \frac{\partial \mu_{n}}{\partial N_{n}}
\end{array}\right] } \\
= & {\left[\begin{array}{ccc}
2 B_{11}+\frac{1}{\rho_{1}} & \cdots & 2 B_{1 n} \\
\vdots & \ddots & \vdots \\
2 B_{1 n} & \cdots & 2 B_{n n}+\frac{1}{\rho_{n}}
\end{array}\right] }
\end{aligned}
$$

The mixture is stable when all eigenvalues are positive (Solokhin et al., 2002). When on the other hand one of the eigenvalues is not positive, the mixture becomes unstable. The limit of stability is reached when the matrix has one zero eigenvalue and is otherwise positive definite, and is referred to as the spinodal (Heidemann and Khalil, 1980).

\subsubsection{Critical point}

In a binary mixture, the critical point is a stable point which lies on the stability limit (spinodal) (Heidemann and Khalil, 1980) and where the phase boundary and spinodal coincide. In mixtures of more components these critical points become plait points. Critical points and plait points are in general concentrations at which two or more phases are in equilibrium and become indistinguishable (Heidemann, 1994).

There are two criteria that can be used to find plait points. The first one is $\operatorname{det}\left(M_{1}\right)=0$, which is the equation for the spinodal. The other criterion is based on the fact that at the critical point, the third derivative of the free energy should also be zero. For a multicomponent system, this criterion can be reformulated using Legendre transforms as $\operatorname{det}\left(M_{2}\right)=0$ (Beegle et al., 1974; Reid and Beegle, 1977), where: 


$$
M_{2}=\left[\begin{array}{ccc}
\frac{\partial \mu_{1}}{\partial N_{1}} & \cdots & \frac{\partial \mu_{n}}{\partial N_{n}} \\
\vdots & \ddots & \vdots \\
\frac{\partial M_{1}}{\partial N_{1}} & \cdots & \frac{\partial M_{1}}{\partial N_{n}}
\end{array}\right]
$$

Matrix $M_{2}$ is matrix $M_{1}$ with one of the rows replaced by the partial derivatives of the determinant of matrix $M_{1}$. Note: it does not matter which row of the matrix is replaced.

\subsubsection{Phase boundary}

When a mixture becomes unstable and demixes into two or more phases, the chemical potential of each component and the osmotic pressure is the same in all phases (Hill, 1986).

$$
\left\{\begin{array}{c}
\beta \Pi^{I}=\beta \Pi^{I I}=\cdots \\
\beta \mu_{1}^{I}=\beta \mu_{1}^{I I}=\cdots \\
\vdots \\
\beta \mu_{n}^{I}=\beta \mu_{n}^{I I}=\cdots
\end{array}\right.
$$

where the phases are denoted by $I, I I, \ldots$.

For a mixture containing $n$ distinguishable components, that demixes into two phases, this results in $n+1$ equations and $2 \times n$ unknowns. To solve the set of equations without having to fix the concentration of one component and the ratio between the other components for at least one of the phases, we need extra equations. For the extra set of equations, we build on the fact that no particles are lost and no new particles are created during phase separation, and the fact that we assume that the total volume does not change.

For a system that separates into two phases we then obtain:

$$
\rho=\sum_{i}^{2} \rho_{i}=\frac{\sum_{i}^{2} N_{i}}{V}=\frac{\sum_{i}^{2} N_{i}^{I}+\sum_{i}^{2} N_{i}^{I I}}{V^{I}+V^{I I}}
$$

which can be rewritten as (Sturtewagen and van der Linden, 2019a):

$$
\begin{aligned}
\rho & =\alpha^{I} \sum_{i}^{2} \rho_{i}^{I}+\left(1-\alpha^{I}\right) \sum_{i}^{2} \rho_{i}^{I I} \\
& =\sum_{i}^{2} \rho_{i}
\end{aligned}
$$

with 
Table 5.1: Virial coefficients and critical point of polyethylene glycol (PEO35) and dextran (D100) from experiment by Dewi et al. (2020) used in the fitting for polydisperse virial coefficient matrix.

\begin{tabular}{ccc}
\hline B $_{\text {PEO35 }}\left(\mathbf{m}^{\mathbf{3}} / \mathbf{m o l}\right)$ & $\mathbf{B}_{\text {D100 }}\left(\mathbf{m}^{\mathbf{3}} / \mathbf{m o l}\right)$ & Critical point $\left(\mathbf{m o l} / \mathbf{m}^{\mathbf{3}}\right)$ \\
\hline 4.74 & 1.31 & $(0.40,1.06)$ \\
\hline
\end{tabular}

$$
\alpha^{I}=\frac{V^{I}}{\sum_{i}^{f} V^{i}}
$$

in which $f$ denotes the number of phases.

This results in the following set of equations:

$$
\left\{\begin{aligned}
\beta \Pi^{I} & =\beta \Pi^{I I}=\cdots \\
\beta \mu_{1}^{I} & =\beta \mu_{1}^{I I}=\cdots \\
\vdots & \\
\beta \mu_{n}^{I} & =\beta \mu_{n}^{I I}=\cdots \\
\rho_{1} & =\alpha^{I} \rho_{1}^{I}+\cdots+\left(1-\sum_{i}^{f-1} \alpha^{i}\right) \rho_{1}^{f} \\
\vdots & \\
\rho_{n} & =\alpha^{I} \rho_{n}^{I}+\cdots+\left(1-\sum_{i}^{f-1} \alpha^{i}\right) \rho_{n}^{f}
\end{aligned}\right.
$$

With this set of equations, we have $2 \times n+1$ unknowns and $2 \times n+1$ equations for mixtures that separate into two phases. Therefore, the set of equations given by Eq. (5.14) allows for calculating the concentration of each component in each of the phases for any given parent concentration, given that the mixture will demix at this concentration.

\subsection{Methods}

Size exclusion chromatography with multi-angle laser light scattering (SEC-MALLS) for both polyethylene oxide (PEO35) and dextran (D100) was performed by NIZO Food Research to obtain the molar mass and size distribution (size distributions in Figure 5.2, SEC-MALLS molar mass plots in appendix). The experimental critical point and the osmotic virial coefficients for the pure components PEO35 and D100 were taken from Dewi et al. (2020), see Table 5.1. The cross-virial coefficients used in the calculations are the result of a fitting including the polydispersity as follows. 
Both components exhibited polydispersity (see Figure 5.2). The size distributions for both components were binned (the number of bins $(\mathrm{N})$ were 1,2 and 5 for PEO and 1,2 and 10 for dextran). The obtained radii and fractions were taken as a starting point for the fit for the polydisperse virial coefficients. For the fitting, non-additive hard sphere interaction was assumed, where cross-interactions among the (chemically equivalent) PEO sub-components was considered the same for all sizes $\left(\Delta_{P E O 35}\right)$, as was also assumed for the polydisperse (chemically equivalent) dextran $\left(\Delta_{D 100}\right)$. The cross-interactions between sub-components of PEO and the sub-components of dextran was also considered the same for all sizes $\left(\Delta_{P D}\right)$. The virial coefficients for both polydisperse components were fitted to the osmometric measured virial coefficients and the critical point from Table 5.1, using Eqs. (5.7), (5.11) and (5.12) and the sub-component fractions obtained from the binned size distribution, while adjusting the sizes of the sub-components (within a small range) and the non-additivity parameters $\left(\Delta_{P E O 35}, \Delta_{D 100}\right.$, and $\left.\Delta_{P D}\right)$. The obtained matrix of virial coefficients was used to calculate the phase boundary and fractionation of the polydisperse mixture. The resulting phase diagram was compared to the experimental phase diagram from Dewi et al. (2020). A fitting was considered good when the critical point of the polydisperse mixture was on the two-phase boundary and the dilution line trough the critical point reached equal volume at the critical point.

\subsection{Results and discussion}

Both PEO and dextran showed significant polydispersity. The distribution for PEO is relatively narrow, slightly bimodal and has a small tail, while the distribution for dextran has more peaks and a considerable fat tail. Based on the research we did on the effect of polydispersity on the phase behavior of a binary mixture of hard spheres (Sturtewagen and van der Linden, 2019a) we hypothesize that especially the larger components of the dextran will influence the phase behavior. The osmometric number average virial coefficient of PEO is considerably larger than the number average virial coefficient of dextran (Table 5.1), even though the radius of the PEO molecules was not larger. This indicates that the repulsive depletion interaction between PEO molecules is larger than the repulsive interaction between dextran molecules.

In order to determine which effect has a stronger influence on the phase behavior (the large size difference between dextran molecules, or the higher repulsive interaction between PEO molecules), we stepwise introduce more polydispersity into our theoretical calculations of the phase diagram. We will compare these calculations to the experimental phase diagram from Dewi et al. (2020). First we introduce polydispersity only for PEO (Section 5.4.1), 


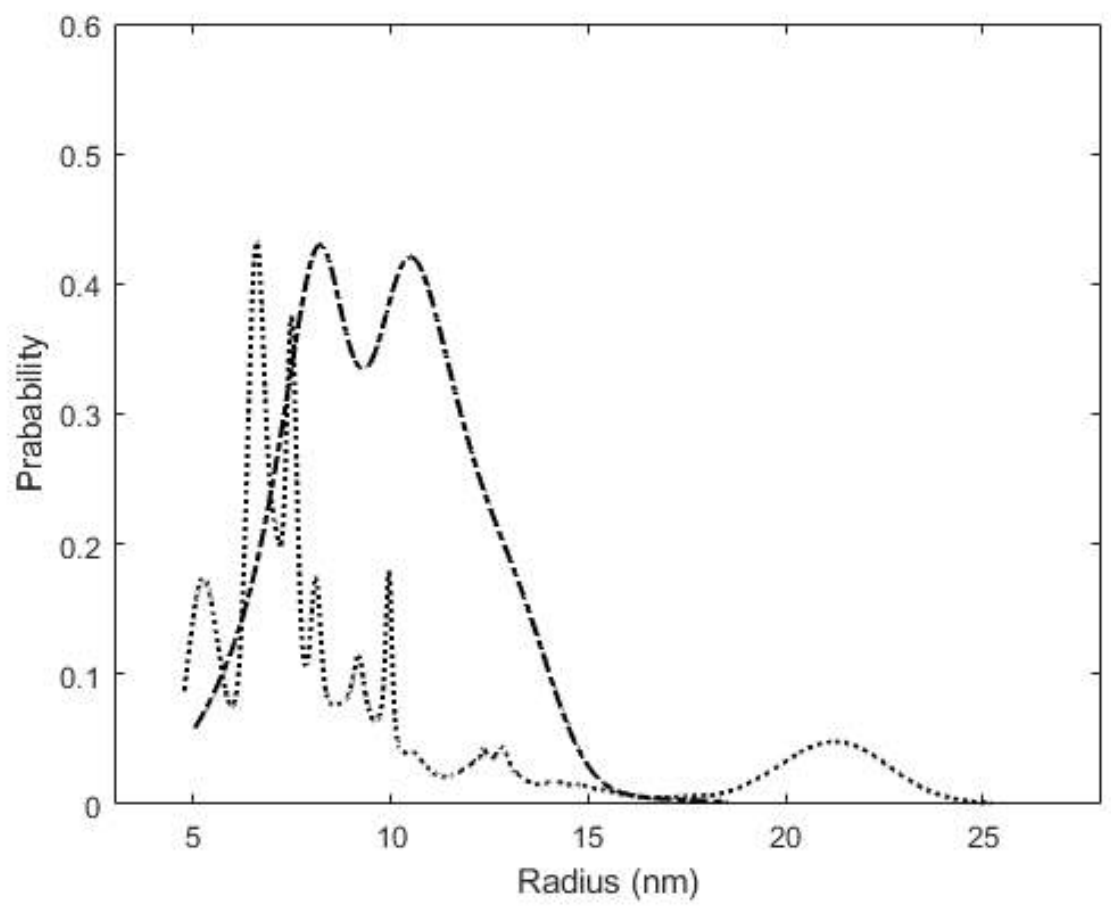

Figure 5.2: Size distribution of PEO 35 (dashed line) and Dextran 100 (dotted line).

subsequently we introduce polydispersity only for dextran (Section 5.4.2), and finally we introduce polydispersity for both PEO and dextran (Section 5.4.3).

\subsubsection{Only PEO polydisperse}

We started by subdividing the distribution from Figure $\mathbf{5 . 2}$ for PEO into two bins with the same bin width. Because the distribution for PEO has a small tail, this resulted in a slightly skewed distribution, see Figure 5.5. The spinodal bends towards the $x$-axis, indicating that it would theoretically become possible for PEO to demix into two phases without the addition of dextran, creating a system with a phase enriched in the smaller component of PEO and a phase enriched in the larger component of PEO. Closer to the critical point, the spinodal matches the spinodal of the monodisperse fitting. The two-phase boundary of the polydisperse fitting near the critical point is close to the phase boundary of the monodisperse mixture. Further away from the critical point, things start to deviate. At low concentrations of PEO, the phase boundary shifts to slightly lower concentrations of dextran. This result is in line with our earlier work on polydisperse hard spheres in solution (Sturtewagen and van der Linden, 2019a), where the larger components of a polydisperse mixture push the phase boundary to lower concentrations of this component at the depleted side of this component. At higher concentrations of PEO, the two-phase boundary bends towards the $\mathrm{x}$-axis. The boundary has a noticeable nod, indicating a drastic change in the 


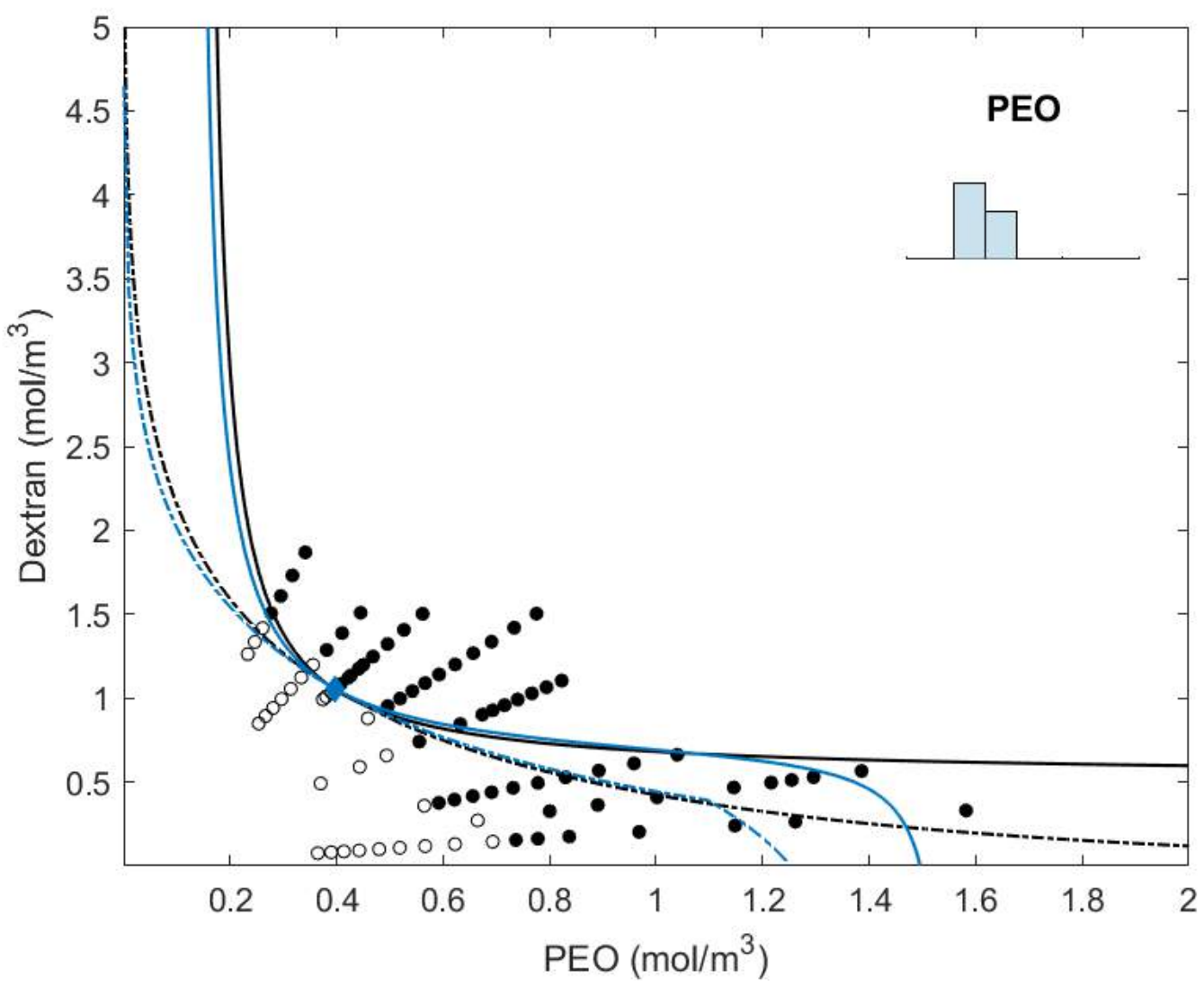

Figure 5.3: Phase diagram for PEO $(\mathrm{N}=2)$ and dextran $(\mathrm{N}=1)$. PEO is sub-divided into two subcomponents, distribution in inset. Dextran is monodisperse. Blue lines are theoretical prediction for the polydisperse mixture, black lines are from the fitting from Dewi et al. (2020). Circles are from the experiment from Dewi et al. (2020). Open circles $(\bigcirc)$ indicate one-phase system, closed circle $(\bullet)$ indicate two-phase system. The diamond $(\checkmark)$ is the critical point. Full line indicates the spinodal. Dashed line indicates the two-phase boundary.

fractionation of the mixture upon demixing (discussed below in Section 5.4.4). Below the nod, the system has a phase enriched in the small component of PEO and a phase enriched in the large concentration of PEO. A mixture with pair-wise interactions such as in this system can in general demix into three phases, however, not at this particular distribution of PEO sub-components. This was found by Sturtewagen and van der Linden (2019b), where three phase systems are described for polydisperse binary mixtures of hard spheres. The change in the position of the binodal for this mixture, is not enough to explain the experimentally demixed mixtures at low concentration of dextran.

Next we subdiveded the PEO into five bins of equal binwidth, see Figure 5.4. The spinodal now bends towards the $x$-axis at lower concentrations of PEO, indicating that if PEO would be the only polydisperse component in the system, the incompatibility among the PEO sub-components would be high enough for the PEO to demix by itself at lower concentrations. Compared to the previous fitting, the polydisperse sub-components push the binodal closer 


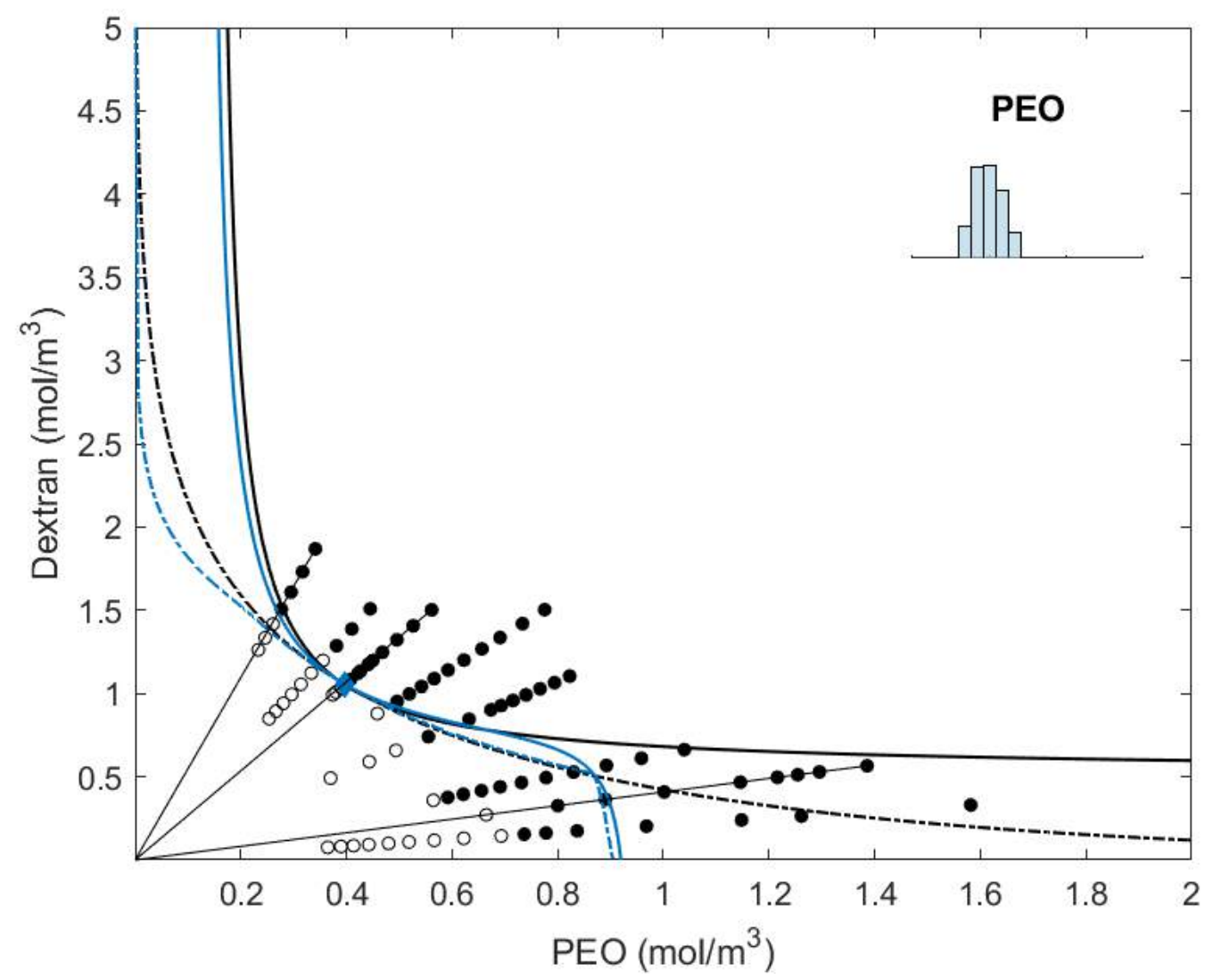

Figure 5.4: Phase diagram for PEO $(\mathrm{N}=5)$ and dextran $(\mathrm{N}=1)$. PEO is sub-divided into five subcomponents, distribution in inset. Dextran is monodisperse. Blue lines are theoretical prediction for the polydisperse mixture, black lines are from the fitting from Dewi et al. (2020). Circles are from the experiment from Dewi et al. (2020). Open circles $(\bigcirc)$ indicate one-phase system, closed circle $(\bullet)$ indicate two-phase system. The diamond $(\bullet)$ is the critical point. Full line indicates the spinodal. Dashed line indicates the two-phase boundary. Three dilution lines are indicated (discussed more in detail in the section Section 5.4.4, see also Table 5.2).

to the $y$-axis at the depleted side of the binodal. At the PEO enriched side of the binodal, the binodal bends towards the $x$-axis. The considerable nod in the binodal indicates a change in the fractionation among the sub-components (see also Table 5.2). A mixture with pair-wise interactions such as in this system can demix into more than two phases, however, the distribution of sub-components of this mixture make this phase boundary unattainable within the considered concentration range. The fitting for this mixture explains some more points of the phase boundary, however there are still a considerable amount of experimental demixed samples that are not explained by considering the polydispersity of PEO alone. 


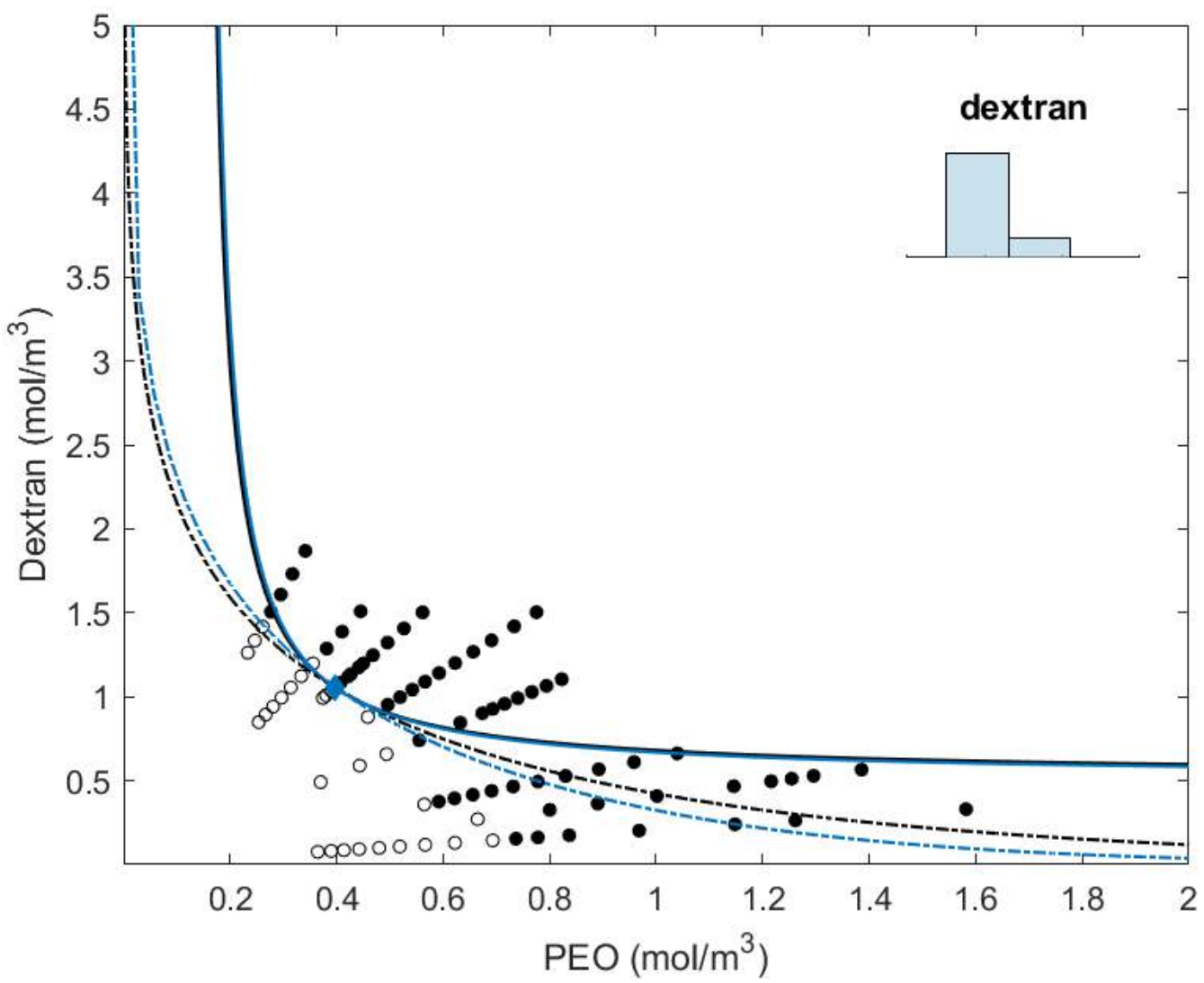

Figure 5.5: Phase diagram for PEO $(\mathrm{N}=1)$ and dextran $(\mathrm{N}=2)$. Dextran is sub-divided two sub-components, distribution in inset. PEO is monodisperse. Blue lines are theoretical prediction for the polydisperse mixture, black lines are from the fitting from Dewi et al. (2020). Circles are from the experiment from Dewi et al. (2020). Open circles $(\bigcirc)$ indicate one-phase system, closed circle (๑) indicate two-phase system. The diamond $(\downarrow)$ is the critical point. Full line indicates the spinodal. Dashed line indicates the two-phase boundary.

\subsubsection{Only dextran polydisperse}

Just as in the previous section, we first sub-divide dextran into two bins with the same bin width. Dextran has a larger tail compared to PEO (Figure 5.2), resulting in a more skewed distribution (Figure 5.5). For this fitting, the difference between the monodisperse and the polydisperse spinodal is negligible. The small polydispersity causes the binodal to shift to lower concentration of dextran at the PEO enriched side and to slightly higher concentration of PEO at the dextran enriched side. The differences are small and only explain a couple of experimental points more compared to the previous mixtures. Mixtures with this pair-wise interaction will at no concentration demix into more than two-phases.

When sub-dividing the dextran into ten bins of equal bin width (of a similar bin width as the PEO sub-divided in 5 bins), we capture more details of the polydispersity into our fitting. This causes the spinodal to bend towards the $y$-axis (Figure 5.6) at higher dextran concentrations. Indicating that at higher concentrations of dextran the mixture can demix 


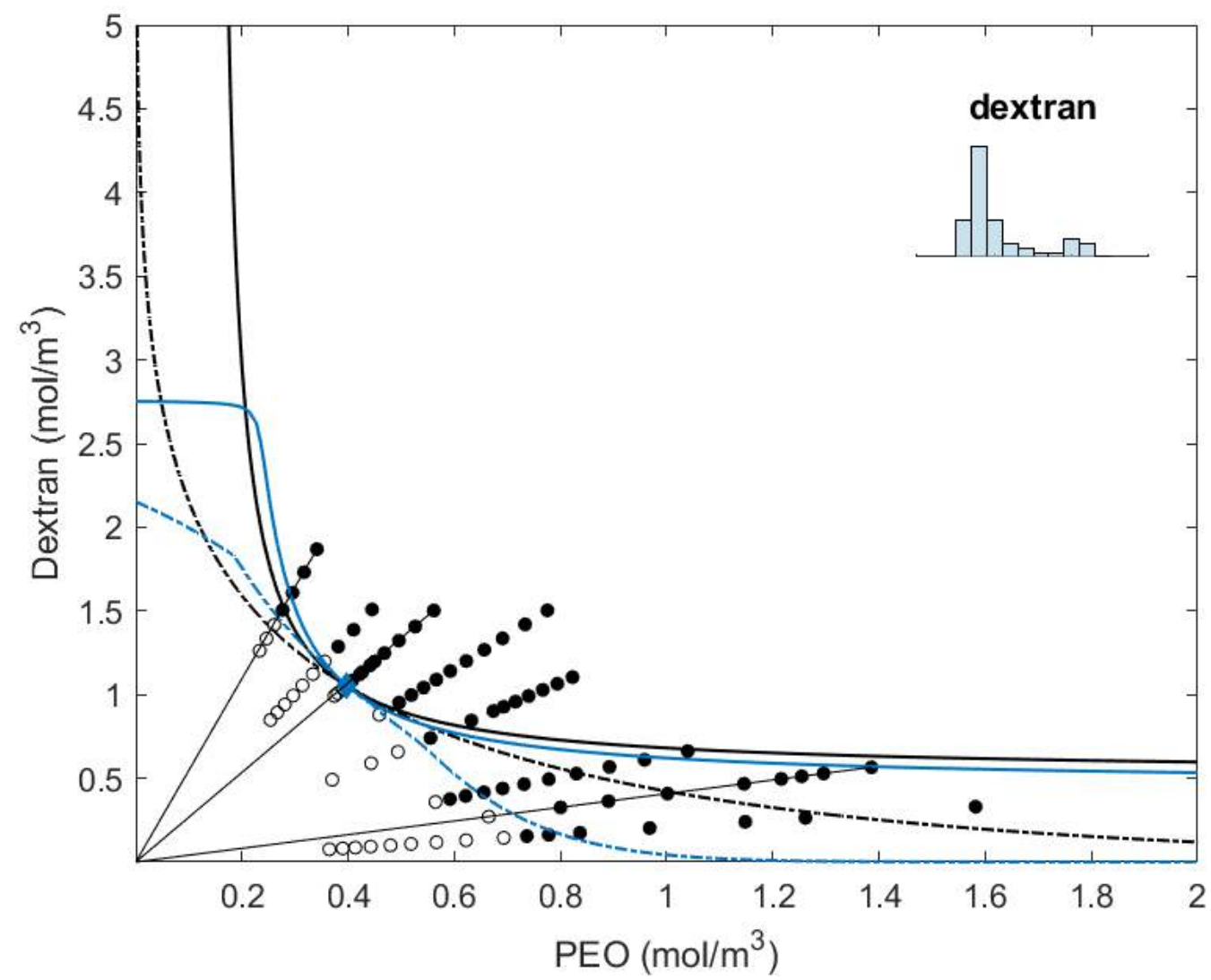

Figure 5.6: Phase diagram for PEO $(\mathrm{N}=1)$ and dextran $(\mathrm{N}=10)$. Dextran is sub-divided into ten sub-components, distribution in inset. PEO is monodisperse. Blue lines are theoretical prediction for the polydisperse mixture, black lines are from the fitting from Dewi et al. (2020). Circles are from the experiment from Dewi et al. (2020). Open circles $(\bigcirc)$ indicate one-phase system, closed circle $(\bullet)$ indicate two-phase system. The diamond $(\bullet)$ is the critical point. Full line indicates the spinodal. Dashed line indicates the two-phase boundary. Three dilution lines are indicated (discussed more in detail in the section Section 5.4.4, see also Table 5.3).

into two phases of dextran. The binodal of the mixture with this fitting changes also quite drastically. At the dextran enriched side, the phase boundary first shifts towards higher PEO concentrations and bends then with increasing dextran concentrations towards the axis. The curve has a noticeable nod. This indicates a regime change in the demixing. At the PEO depleted side of the curve, the two-phase demixing is with a phase enriched in PEO and a phase enriched in dextran, but a phase enriched in the smaller components of dextran and a phase enriched in the larger components of dextran, similarly as described for PEO above. At the PEO enriched side of the curve, the binodal shifts towards lower concentrations of dextran while asymptotically approaching the x-axis. Also components with this pairwise interaction can demix into more than two phases, however just as in the previous cases, the concentrations required for multi-phase demixing are unattainable with this particle distribution. The fitting captures more of the experimentally phase separated 
samples with low dextran concentration (filled circles in the plot), compared to the previous fittings. This indicates that our hypothesis that the polydispersity of the dextran would be a driving factor in the demixing of these samples is valid.

Döbert et al. (1995) constructed a consistent osmotic virial equation to predict the phase behavior of a different type of polydisperse dextran and monodisperse PEO. They obtained an average molecular weight osmotic virial coefficient for the different polymer chains and compared their result to predictions assuming a binary monodisperse system. They report a better fit when polydispersity is taken into account. When predicting the phase separation for mixtures close to the phase boundary, they also report that the binodal shifts closer to the axis in the dextran depleted side of the binodal. They report a strong influence of the volume fraction of the phase enriched in dextran on the shape of the phase boundary and the fractionation of the polydisperse dextran.

\subsubsection{Both PEO and dextran polydisperse}

Next we introduce polydispersity for both PEO and dextran into our fitting. We subdivided the PEO and the dextran into two bins, of different bin width for each polymer. This results in the same distribution as in Figures $\mathbf{5 . 3}$ and 5.5. The resulting phase diagram is a combination fo the phase diagrams of the previous sections. The spinodal matches the spinodal of the monodisperse fitting very closely. Only at increasing PEO concentrations, the spinodal bends towards the $x$-axis. The higher repulsive interaction among the PEO sub-components results in instability at higher PEO concentrations. Even without the addition of dextran it becomes possible for PEO to demix. At both the PEO enriched side of the phase boundary as well as at the dextran enriched side of the phase boundary, the boundary shifts towards lower concentrations. This a combination of what we saw when only one component was polydisperse. Mixtures with this type of pairwise interaction can theoretically demix into multiple phases, but the concentrations required for this type of multi-phase system are unattainable with these size distributions.

Finally we combine the more detailed distribution of sub-components from Figures $\mathbf{5 . 4}$ and 5.6. PEO is sub-divided into five sub-components and dextran is sub-divided into ten sub-components. The resulting phase-diagram is again a combination from what we saw before when we only considered the polydispersity of one component. At concentrations where both components are present in a significant amount, e.g. near the critical point and higher concentrations, the spinodal follows the spinodal of the monodisperse fitting. At these concentrations the bulk interactions between all sub-components of PEO and dextran govern the interaction and resulting instability of the mixture. At the PEO depleted side of the phase diagram, the spinodal bends towards the $y$-axis, indication that at larger 


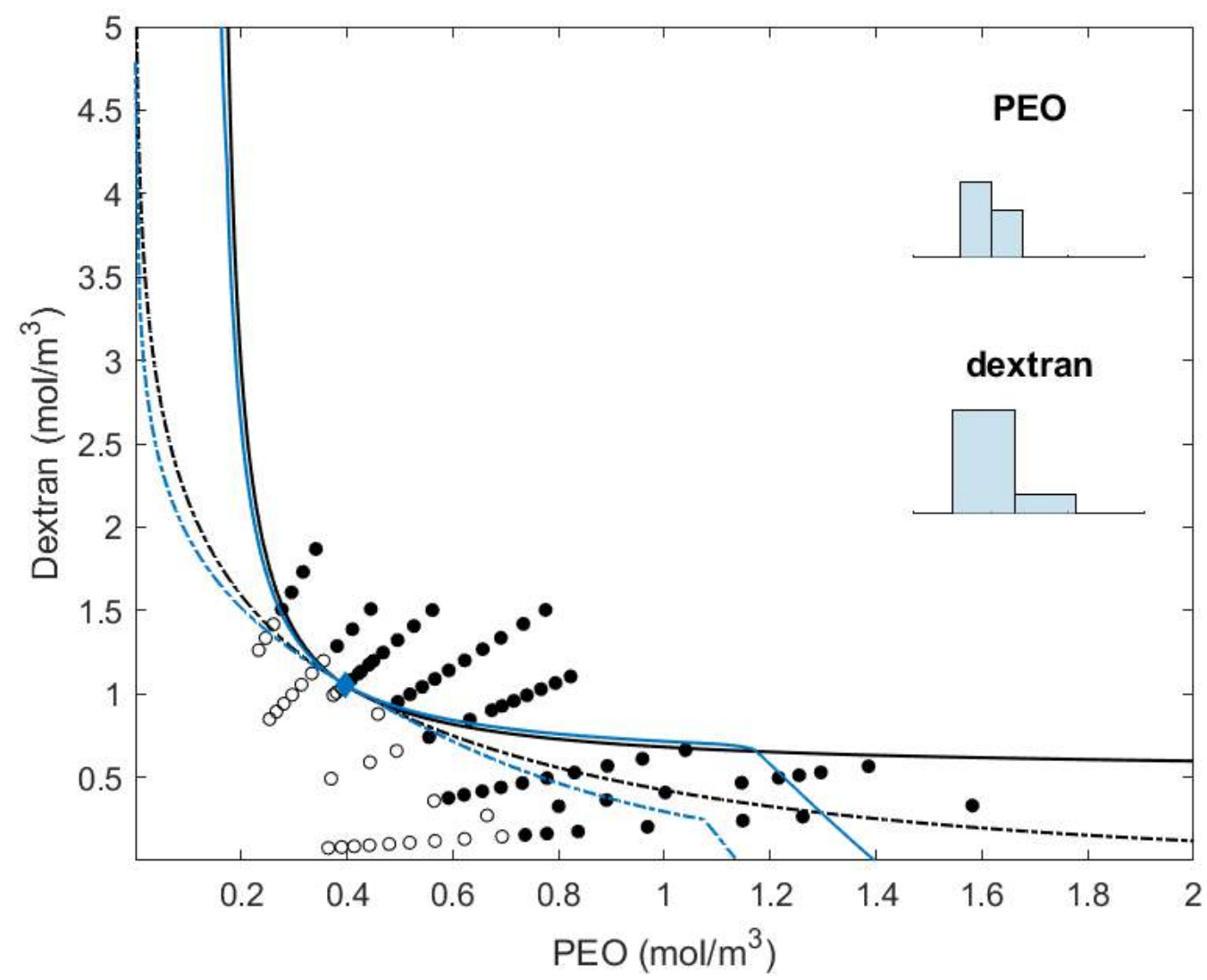

Figure 5.7: Phase diagram for PEO $(\mathrm{N}=2)$ and dextran $(\mathrm{N}=2)$. Both $\mathrm{PEO}$ and dextran are sub-divided into two sub-components, distribution in inset. Blue lines are theoretical prediction for the polydisperse mixture, black lines are from the fitting from Dewi et al. (2020). Circles are from the experiment from Dewi et al. (2020). Open circles $(\bigcirc)$ indicate one-phase system, closed circle (๑) indicate two-phase system. The diamond $(\checkmark)$ is the critical point. Full line indicates the spinodal. Dashed line indicates the two-phase boundary.

concentrations of dextran, dextran can become instable by itself resulting in demixing between the smaller and larger dextran sub-components. When a small amount PEO is added to dextran, first the larger sub-components of the PEO will result in demxing. This causes the phase boundary to shift to lower concentrations compared to when both components are considered monodisperse. Looking at the PEO enriched side of the phase diagram, higher concentrations of PEO are required for PEO to become unstable by itself. This is because when fitting the pair wise interactions for the system, the number average virial coefficients, as well as the position of the critical point where taken into account. Taking more sub-components into account for the whole system allowed for the distribution of both components to play a more important role. When comparing the experimental phase diagram to the phase boundary of the polydisperse fitted system, the polydispersity explains a lot more of the experimentally demixed samples than the monodisperse fitted system. It would be interesting to investigate also a bit more samples at high dextran concentration 


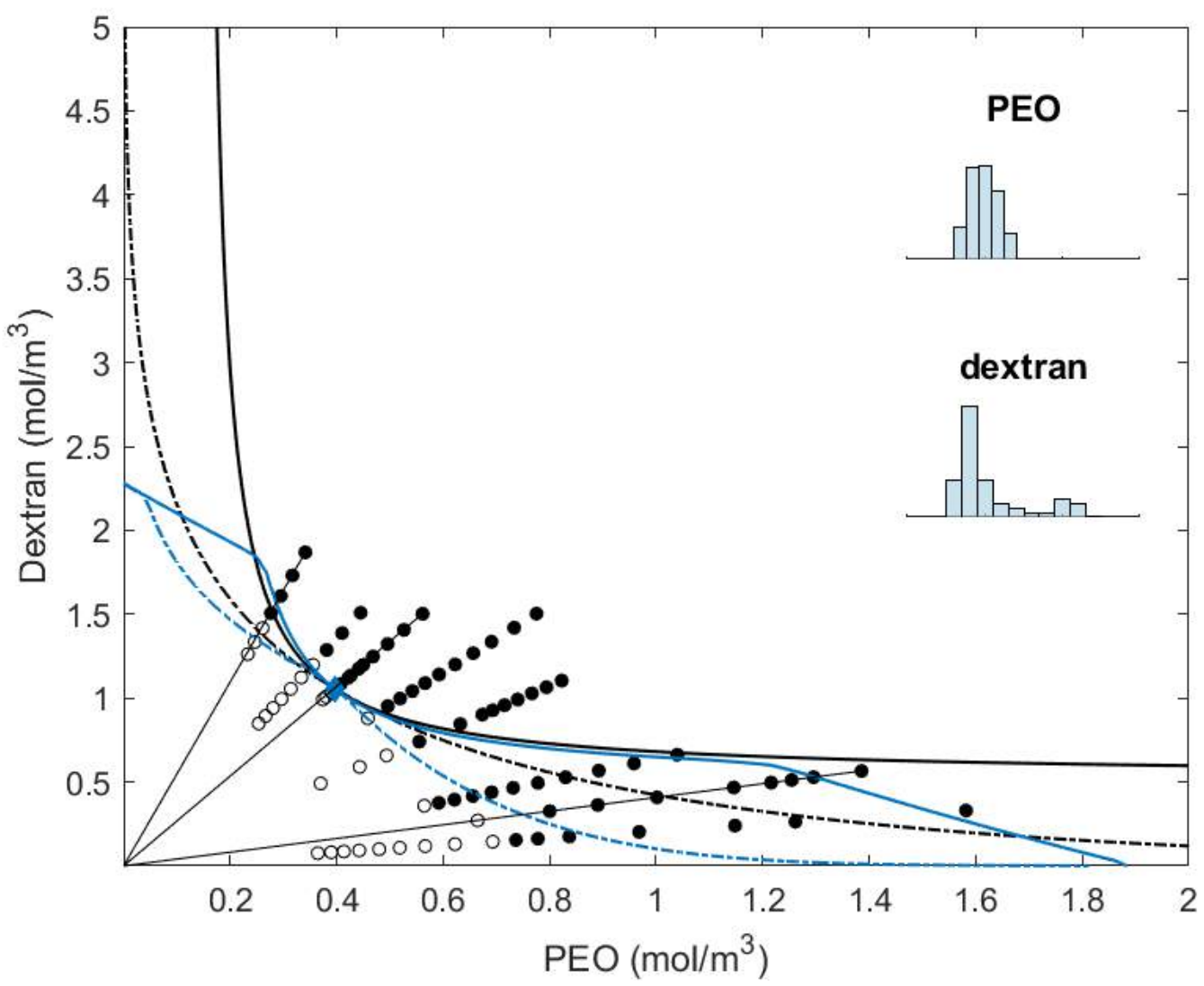

Figure 5.8: Phase diagram for PEO $(\mathrm{N}=5)$ and dextran $(\mathrm{N}=10)$. PEO is sub-divided into five sub-components, Dextran is sub-divided into ten sub-components, distribution in inset. Blue lines are theoretical prediction for the polydisperse mixture, black lines are from the fitting from Dewi et al. (2020). Circles are from the experiment from Dewi et al. (2020). Open circles $(\bigcirc)$ indicate one-phase system, closed circle $(\bullet)$ indicate two-phase system. The diamond $(\bullet)$ is the critical point. Full line indicates the spinodal. Dashed line indicates the two-phase boundary. Three dilution lines are indicated (discussed more in detail in the section Section 5.4.4, see also Table 5.4).

side of the phase boundary to compare to the theoretical predictions.

\subsubsection{Fractionation}

We compare for three systems (Figures 5.4, 5.6 and 5.8) three dilution lines. One dilution line with high dextran concentrations, one through the critical point, and finally one with high PEO concentrations in Tables 5.2 to 5.4. We compare these dilution lines to the experimental system of Dewi et al. (2020).

In Table 5.2, only PEO is considered as polydisperse. In general, when the phase is enriched in PEO, the distribution resembles the parent phase more, while when the phase is depleted in PEO, the distribution contains more of the smaller size PEO molecules. This changes drastically at the lower concentrations of the third dilution line (last few samples of 
the table). For these samples, the mixtures contained only low concentrations of dextran. The demixing for these samples is governed by the incompatibility among the polydisperse PEO subcomponents, and not by the incompatibility between PEO and dextran. Both phases contain nearly the same amount of dextran, but have a drastically different PEO distribution. The volume of the phase enriched in the larger sub-components of PEO is only very small. Gaube et al. (1993) varied the apparent polydispersity of PEO by combining different PEO sizes in different ratios, while keeping the dextran type the same for a variety of mixtures. They found that mainly the short-chain PEO distributes towards the dextran rich phase upon demixing, while the average molecular weight of the phase enriched in PEO does not significantly change. This results is in line with our theoretical predictions.

In Table 5.3, only dextran is considered as polydisperse. Also in this case, the phase depleted in dextran contains mainly the smaller size sub-components of dextran. The lowest dilution line has a relatively high concentration of PEO and a low concentration of dextran. Here the polydispersity of dextran plays an important role in the demixing of both components. A small amount of high molecular weight dextran becomes immiscible with the concentrated PEO, this was also reported by Zhao et al. (2016). This results in a large phase enriched in PEO and the low molecular weight dextran and a very small $(\alpha<0.15)$ phase enriched in the large molecular weight fraction of dextran (Table 5.3). With decreasing parent concentration, the dextran enriched phase decreases in volume and the fraction of large molecular weight dextran increases. While the distribution of dextran in the PEO enriched phase starts to resemble the distribution of the parent phase more and more.

In the last table (Table 5.4), both PEO and dextran are considered polydisperse. Just as with the phase diagram (Figure 5.8), taking the polydispersity of both components into account results in a combination of the patterns we saw when taking only the polydispersity of one of them into account. A general pattern that returns here is that smaller components more easily partition into the their depleted phase, while the larger components are more incompatible with the other component and partition to the enriched phase. This is also something that was experimentally shown by Gaube et al. (1993) and Edelman et al. (2003). Especially when decreasing the concentration along the dilution line through the critical point, the distribution in each phase continuously resembles the parent distribution more and more. This pattern does not emerge when following a dilution line further away from the critical point. Especially for the lower dilution line, with high PEO concentrations, this is not true, as the phase enriched in dextran contains relatively more and more larger dextran molecules when decreasing the concentration. 
Table 5.2: Phase separation of polydisperse PEO $(N=5)$ and polydisperse dextran $(N=10)$ for the indicated dilution lines from Figure 5.4, distribution of polydisperse component in each phase and volume fracion of each phase $(\alpha)$, concentrations in $\left(\mathrm{mol} / \mathrm{m}^{3}\right)$, concentrations that also demixed in two phases in the experiments of Dewi et al. (2020) are indicted by $\boldsymbol{\bullet}$, mixtures that did not demix are indiceted by $\bigcirc$. Only compositions that demix according to the fitted interactions are represented. Red line: number average particle size.

\begin{tabular}{|c|c|c|}
\hline Parent phase & Phase enriched in PEO & Phase enriched in dextran \\
\hline$(0.34,1.87), \bullet$ & $(1.12,0.36), \alpha: 0.24$ & $(0.09,2.35), \alpha: 0.76$ \\
\hline$(0.32,1.73), \bullet$ & $(0.98,0.43), \alpha: 0.23$ & $(0.11,2.13), \alpha: 0.77$ \\
\hline$(0.30,1.61), \bullet$ & $(0.84,0.51), \alpha: 0.21$ & $(0.14,1.91), \alpha: 0.79$ \\
\hline$(0.28,1.51), \bullet$ & $(0.70,0.61), \alpha: 0.18$ & $(0.18,1.70), \alpha: 0.82$ \\
\hline$(0.26,1.42), \bigcirc$ & $(0.57,0.73), \alpha: 0.10$ & $(0.22,1.49), \alpha: 0.90$ \\
\hline$(0.56,1.50), \bullet$ & $(1.17,0.34), \alpha: 0.43$ & $(0.08,2.39), \alpha: 0.57$ \\
\hline$(0.53,1.41), \bullet$ & $(1.06,0.39), \alpha: 0.44$ & $(0.10,2.20), \alpha: 0.56$ \\
\hline$(0.50,1.32), \bullet$ & $(0.94,0.46), \alpha: 0.44$ & $(0.13,2.02), \alpha: 0.56$ \\
\hline$(0.47,1.25), \bullet$ & $(0.84,0.53), \alpha: 0.45$ & $(0.16,1.84), \alpha: 0.55$ \\
\hline$(0.45,1.20), \bullet$ & $(0.76,0.59), \alpha: 0.46$ & $(0.18,1.71), \alpha: 0.54$ \\
\hline$(0.44,1.18)$, & $(0.72,0.62), \alpha: 0.46$ & $(0.19,1.65), \alpha: 0.54$ \\
\hline \multicolumn{3}{|c|}{ Continued on next page } \\
\hline
\end{tabular}




\section{Continuation of Table 5.4}

\begin{tabular}{|c|c|c|}
\hline Parent phase & Phase enriched in PEO & Phase enriched in dextran \\
\hline$(0.43,1.13)$, & $(0.65,0.69), \alpha: 0.47$ & $(0.22,1.52), \alpha: 0.53$ \\
\hline$(0.42,1.12), \bullet$ & $(0.62,0.72), \alpha: 0.47$ & $(0.24,1.48), \alpha: 0.53$ \\
\hline & & \\
\hline$(0.41,1.08), \bullet$ & $(0.53,0.83), \alpha: 0.44$ & $(0.28,1.32), \alpha: 0.52$ \\
\hline$(0.40,1.07), \bullet$ & $(0.49,0.89), \alpha: 0.49$ & $(0.31,1.24), \alpha: 0.51$ \\
\hline$(0.40,1.06), \bullet$ & $(0.44,0.96), \alpha: 0.49$ & $(0.35,1.15), \alpha: 0.51$ \\
\hline$(1.39,0.57), \bullet$ & $(1.56,0.21), \alpha: 0.87$ & $(0.04,3.04), \alpha: 0.13$ \\
\hline$(1.30,0.55)$, & $(1.44,0.25), \alpha: 0.89$ & $(0.05,2.81), \alpha: 0.11$ \\
\hline & Hillh & 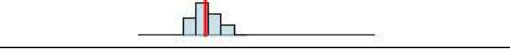 \\
\hline$(1.25,0.51), \bullet$ & $(1.38,0.26), \alpha: 0.90$ & $(0.06,2.71), \alpha: 0.10$ \\
\hline & ताthr & तlth \\
\hline$(1.22,0.50), \bullet$ & $(1.32,0.28), \alpha: 0.91$ & $(0.06,2.61), \alpha: 0.09$ \\
\hline & $\frac{\text { नाllh }}{(121202)}$ & $(000012)$ \\
\hline$(1.15,0.47), \bullet$ & $(1.21,0.33), \alpha: 0.93$ & $(0.08,2.43), \alpha: 0.07$ \\
\hline$(1.00,0.41), \bullet$ & $(1.08,0.41), \alpha: 0.95$ & $(0.61,0.41), \alpha: 0.05$ \\
\hline & -तlh & त्ताी \\
\hline \multirow[t]{2}{*}{$(0.89,0.36), \bullet$} & $(0.91,0.36), \alpha: 0.99$ & $(0.62,0.36), \alpha: 0.01$ \\
\hline & & ח \\
\hline
\end{tabular}


Table 5.3: Phase separation of monodisperse PEO $(N=1)$ and polydisperse dextran $(N=10)$ for the indicated dilution lines from Figure 5.6, distribution of polydisperse component in each phase and volume fracion of each phase $(\alpha)$, concentrations in $\left(\mathrm{mol} / \mathrm{m}^{3}\right)$, concentrations that also demixed in two phases in the experiments of Dewi et al. (2020) are indicted by $\boldsymbol{\bullet}$, mixtures that did not demix are indiceted by $\bigcirc$. Only compositions that demix according to the fitted interactions are represented. Red line: number average particle size.

\begin{tabular}{|c|c|c|}
\hline Parent phase & Phase enriched in PEO & Phase enriched in dextran \\
\hline$(0.34,1.87), \bullet$ & $\begin{array}{c}(1.10,0.61), \alpha: 0.23 \\
.\end{array}$ & $(0.12,2.24), \alpha: 0.77$ \\
\hline$(0.32,1.73), \bullet$ & $\begin{array}{c}(0.96,0.61), \alpha: 0.21 \\
.\end{array}$ & $(0.15,2.01), \alpha: 0.79$ \\
\hline$(0.30,1.61), \bullet$ & $\begin{array}{l}(0.83,0.76), \alpha: 0.17 \\
.\end{array}$ & $(0.19,1.78), \alpha: 0.83$ \\
\hline$(0.28,1.51), \bullet$ & $(0.70,0.85), \alpha: 0.09$ & $(0.24,1.57), \alpha: 0.91$ \\
\hline$(0.56,1.50), \bullet$ & $(1.13,0.58), \alpha: 0.44$ & $(0.11,2.23), \alpha: 0.55$ \\
\hline$(0.53,1.41), \bullet$ & $\begin{array}{c}(1.02,0.64), \alpha: 0.45 \\
.\end{array}$ & 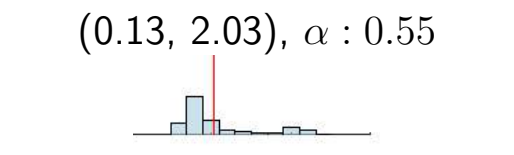 \\
\hline$(0.50,1.32), \bullet$ & $\begin{array}{c}(0.91,0.69), \alpha: 0.45 \\
.\end{array}$ & $(0.16,1.84), \alpha: 0.55$ \\
\hline$(0.47,1.25), \bullet$ & $\begin{array}{c}(0.81,0.74), \alpha: 0.46 \\
.\end{array}$ & $(0.19,1.67), \alpha: 0.54$ \\
\hline$(0.45,1.20), \bullet$ & $\begin{array}{c}(0.73,0.78), \alpha: 0.46 \\
.\end{array}$ & 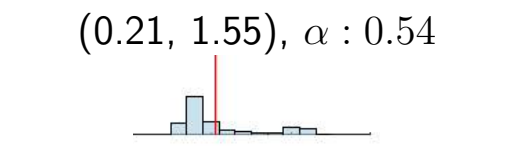 \\
\hline$(0.44,1.18), \bullet$ & $(0.70,0.81), \alpha: 0.46$ & $(0.22,1.49), \alpha: 0.54$ \\
\hline$(0.43,1.13), \bullet$ & $(0.63,0.85), \alpha: 0.47$ & 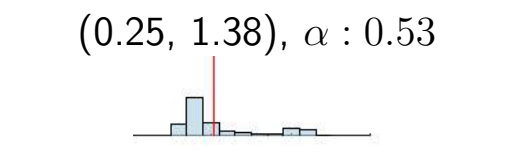 \\
\hline \multicolumn{3}{|c|}{ Continued on next page } \\
\hline
\end{tabular}




\section{Continuation of Table 5.3}

\begin{tabular}{|c|c|c|}
\hline Parent phase & Phase enriched in PEO & Phase enriched in dextran \\
\hline$(0.42,1.12)$, & 3), $\alpha: 0.47$ & $(0.26,1.35), \alpha: 0.53$ \\
\hline$(0.41,1.08), \bullet$ & $(0.52,0.93), \alpha: 0.48$ & 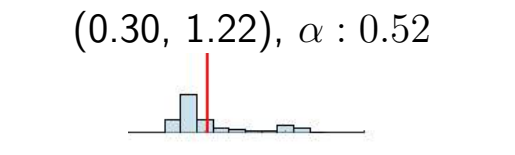 \\
\hline$(0.40,1.07), \bullet$ & $(0.48,0.96), \alpha: 0.48$ & $(0.33,1.17), \alpha: 0.52$ \\
\hline$(0.40,1.06), \bullet$ & $(0.44,1.00), \alpha: 0.49$ & 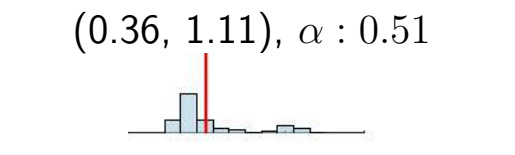 \\
\hline$(1.39,0.57), \bullet$ & $\begin{array}{c}(1.54,0.35), \alpha: 0.89 \\
\text { r.thn }\end{array}$ & $(0.04,2.35), \alpha: 0.11$ \\
\hline$(1.30,0.55)$, & $\begin{array}{c}(1.43,0.37), \alpha: 0.91 \\
\text {. }\end{array}$ & $(0.05,2.10), \alpha: 0.09$ \\
\hline$(1.25,0.51), \bullet$ & $\begin{array}{c}(1.37,0.37), \alpha: 0.91 \\
\text {. }\end{array}$ & $(0.05,1.99), \alpha: 0.09$ \\
\hline$(1.22,0.50)$, & $\begin{array}{l}(1.22,0.37), \alpha: 0.92 \\
.\end{array}$ & $(0.05,1.89), \alpha: 0.08$ \\
\hline$(1.15,0.47), \bullet$ & $\begin{array}{c}(1.23,0.37), \alpha: 0.93 \\
\end{array}$ & $(0.06,1.71), \alpha: 0.07$ \\
\hline$(1.00,0.41), \bullet$ & $(1.04,0.37), \alpha: 0.96$ & $(0.07,1.37), \alpha: 0.04$ \\
\hline$(0.89,0.36), \bullet$ & $(0.90,0.35), \alpha: 0.98$ & $(0.06,1.09), \alpha: 0.02$ \\
\hline$(0.80,0.33), \bullet$ & $(0.80,0.32), \alpha: 1.00$ & $(0.03,0.81), \alpha: 0.00$ \\
\hline
\end{tabular}


Table 5.4: Phase separation of polydisperse PEO $(N=5)$ and polydisperse dextran $(N=10)$ for the indicated dilution lines from Figure 5.8, distribution of polydisperse component in each phase and volume fracion of each phase $(\alpha)$, concentrations in $\left(\mathrm{mol} / \mathrm{m}^{3}\right)$, concentrations that also demixed in two phases in the experiments of Dewi et al. (2020) are indicted by $\boldsymbol{\bullet}$, mixtures that did not demix are indiceted by $\bigcirc$. Only compositions that demix according to the fitted interactions are represented. Red line: number average particle size.

\begin{tabular}{ll}
\hline Parent phase Phase enriched in PEO Phase enriched in dextran \\
\hline$(0.34,1.87), \bullet$ & $(0.96,0.45), \alpha: 0.17$
\end{tabular}




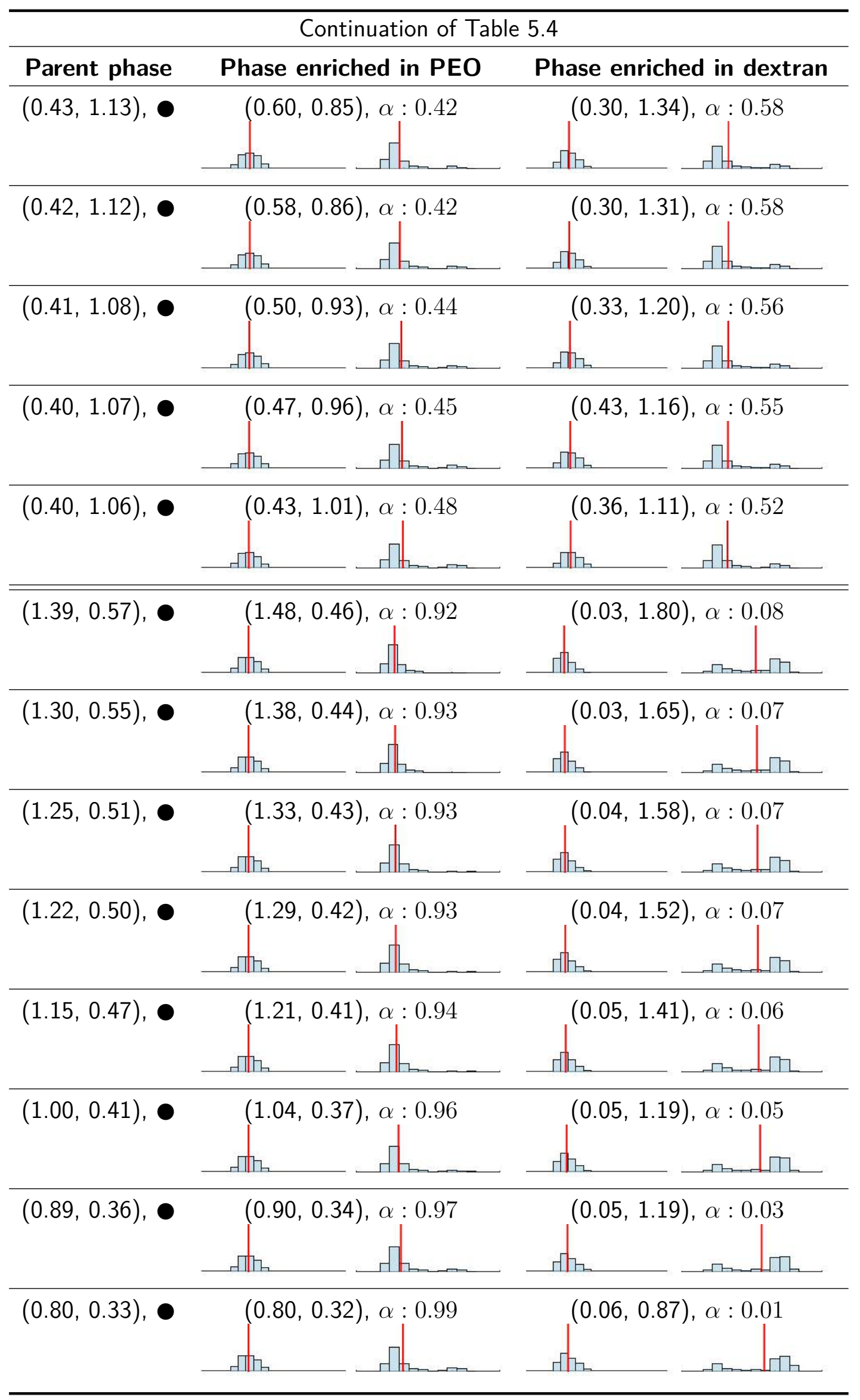




\subsection{Conclusion}

Polydispersity plays an important role in the phase behavior of the colloids PEO and dextran. The components with a larger than average molecular weight govern the transition between one and two phases close to the phase boundary in their respective depleted concentration ranges. This causes a decrease in miscibility and a shift of the phase boundary to lower concentrations. This causes drastic changes to the shape of the phase boundary. When both components are polydisperse, the phase boundary drastically changes shape and changes from a U-shape to a W-shape. Not only the phase boundary changes shape, also the spinodal has different boundaries depending on the polydispersity. Even though multi-phase separation for mixtures with the fitted interactions are possible, the particle size distributions make concentrations resulting in multi-phase systems unattainable.

Upon demixing, the distribution of polydisperse components changes in each phase. This fractionation is dependent on the parent distribution, the pair-wise interaction between the components of the same type, the pair-wise interaction of the components of a different type, and the concentration of both components in the parent mixture.

Our method of incorporating polydispersity allows for a more precise prediction of the phase boundary compared to assuming monodispersity, especially in the meta stable region. Next to that our method allows for prediction of the concentration and fractionation of each component in each phase depending on the parent concentration as well as the volume fraction of said phases.

The best fit with the available data to the experimental data was when dextran was polydisperse and PEO was monodisperse. 


\subsection{Bibliography}

P.-Å. Albertsson. Partition of cell particles and macromolecules in polymer two-phase systems. Advances in Protein Chemistry, 24(C):309-341, 1970. ISSN 00653233. doi: 10.1016/S0065-3233(08)60244-2.

B. L. Beegle, M. Modell, and R. C. Reid. Thermodynamic stability criterion for pure substances and mixtures. AIChE Journal, 20(6):1200-1206, 1974. ISSN 0001-1541. doi: 10.1002/aic.690200621.

B. P. C. Dewi, E. van der Linden, A. Bot, and P. Venema. Second order virial coefficients from phase diagrams. Food Hydrocolloids, 101:105546, 2020. ISSN 0268005X. doi: 10.1016/j.foodhyd.2019.105546.

M. Dijkstra, R. van Roij, and R. Evans. Phase diagram of highly asymmetric binary hardsphere mixtures. Physical Review E - Statistical Physics, Plasmas, Fluids, and Related Interdisciplinary Topics, 59(5):5744-5771, 1999. ISSN 1063651X. doi: 10.1103/PhysRevE.59.5744.

F. Döbert, A. Pfennig, and M. Stumpf. Derivation of the Consistent Osmotic Virial Equation and its Application to Aqueous Poly(ethylene glycol)-Dextran Two-Phase Systems. Macromolecules, 28(23):7860-7868, 1995. ISSN 15205835. doi: 10.1021/ma00127a037.

E. Dos Santos Tada, W. Loh, and P. D. A. Pessôa-Filho. Phase equilibrium in aqueous two-phase systems containing ethylene oxide-propylene oxide block copolymers and dextran. Fluid Phase Equilibria, 218(2):221-228, 2004. ISSN 03783812. doi: 10.1016/j.fluid.2004.01.001.

M. W. Edelman, E. Van Der Linden, and R. H. Tromp. Phase separation of aqueous mixtures of poly(ethylene oxide) and dextran. Macromolecules, 36(20):7783-7790, 2003. ISSN 00249297. doi: 10.1021/ma0341622.

C. Ersch, E. van der Linden, A. Martin, and P. Venema. Interactions in protein mixtures. Part II: A virial approach to predict phase behavior. Food Hydrocolloids, 52:991-1002, 2016. doi: 10.1016/j.foodhyd.2015.07.021.

J. Gaube, R. Höchemer, B. Keil, and A. Pfennig. Polydispersity Effects in the System Poly(ethylene glycol) + Dextran + Water. Journal of Chemical and Engineering Data, 38(2):207-210, 1993. ISSN 15205134. doi: 10.1021/je00010a005.

R. A. Heidemann. The criteria for thermodynamic stability. AlChE Journal, 21(4):824-826, 1975. ISSN 0001-1541. doi: 10.1002/aic.690210433. 
R. A. Heidemann. The Classical Theory of Critical Points. In Supercritical Fluids, pages 39-64. Springer Netherlands, 1994. doi: 10.1007/978-94-015-8295-7_2.

R. A. Heidemann and A. M. Khalil. The calculation of critical points. AlChE Journal, 26 (5):769-779, 1980. ISSN 15475905. doi: 10.1002/aic.690260510.

T. L. Hill. An Introduction to Statistical Thermodynamics. Dover Publications, New York, 1986. ISBN 9780486652429.

G. Johansson and H. Walter. Partitioning and concentrating biomaterials in aqueous phase systems. International Review of Cytology, 192(1896):33-60, 1999. ISSN 00747696. doi: 10.1016/s0074-7696(08)60521-5.

H. O. Johansson, G. Karlström, F. Tjerneld, and C. a. Haynes. Driving forces for phase separation and partitioning in aqueous two- phase systems. Journal of Chromatography B: Biomedical Applications, 711(1-2):3-17, 1998. ISSN 15726495. doi: 10.1016/S03784347(97)00585-9.

C. H. Kang and S. I. Sandler. Effects of polydispersivity on the phase behavior of the aqueous two-phase polymer systems. Macromolecules, 21(10):3088-3095, 1988. ISSN 0024-9297.

M. Larsson and B. Mattiasson. Characterization of aqueous two-phase systems based on polydisperse phase forming polymers: Enzymatic hydrolysis of starch in a PEG-starch aqueous two-phase system. Biotechnology and Bioengineering, 31(9):979-983, 1988. ISSN 10970290. doi: 10.1002/bit.260310910.

M. Larsson and B. Mattiasson. Molecular Weight Distribution Analysis of Water-Soluble Polymers Using Aqueous Two-Phase Systems. Annals of the New York Academy of Sciences, 672(1 Enzyme Engine):649-656, nov 1992. ISSN 0077-8923. doi: 10.1111/j.17496632.1992.tb35685.x.

H. N. Lekkerkerker and R. Tuinier. Colloids and the Depletion Interaction, volume 833. Springer, 2011. ISBN 978-94-007-1222-5. doi: 10.1007/978-94-007-1223-2.

W. G. McMillan and J. E. Mayer. The Statistical Thermodynamics of Multicomponent Systems. The Journal of Chemical Physics, 13(7):276-305, 1945. ISSN 0021-9606. doi: 10.1063/1.1724036.

R. C. Reid and B. L. Beegle. Critical point criteria in legendre transform notation. A/ChE Journal, 23(5):726-732, 1977. ISSN 15475905. doi: 10.1002/aic.690230515. 
R. Roth, R. Evans, and A. A. Louis. Theory of asymmetric nonadditive binary hard-sphere mixtures. Physical Review E - Statistical Physics, Plasmas, Fluids, and Related Interdisciplinary Topics, 64(5):13, 2001. ISSN 1063651X. doi: 10.1103/PhysRevE.64.051202.

M. A. Solokhin, A. V. Solokhin, and V. S. Timofeev. Phase-equilibrium stability criterion in terms of the eigenvalues of the Hessian matrix of the Gibbs potential. Theoretical Foundations of Chemical Engineering, 36(5):444-446, 2002. ISSN 00405795. doi: 10.1023/A:1020617610644.

L. Sturtewagen and E. van der Linden. Effect of polydispersity on the phase behavior of additive hard spheres in solution, part I. Manuscript submitted, 2019a. URL http://arxiv.org/abs/1912.03143.

L. Sturtewagen and E. van der Linden. Effect of polydispersity on the phase behavior of non-additive hard spheres in solution, part II. Manuscript submitted, 2019b. URL http://arxiv.org/abs/1912.03175.

Z. Zhao, Q. Li, X. Ji, R. Dimova, R. Lipowsky, and Y. Liu. Molar mass fractionation in aqueous two-phase polymer solutions of dextran and poly(ethylene glycol). Journal of Chromatography A, 1452:107-115, 2016. ISSN 18733778. doi: 10.1016/j.chroma.2016.04.075. 


\section{A Figures}

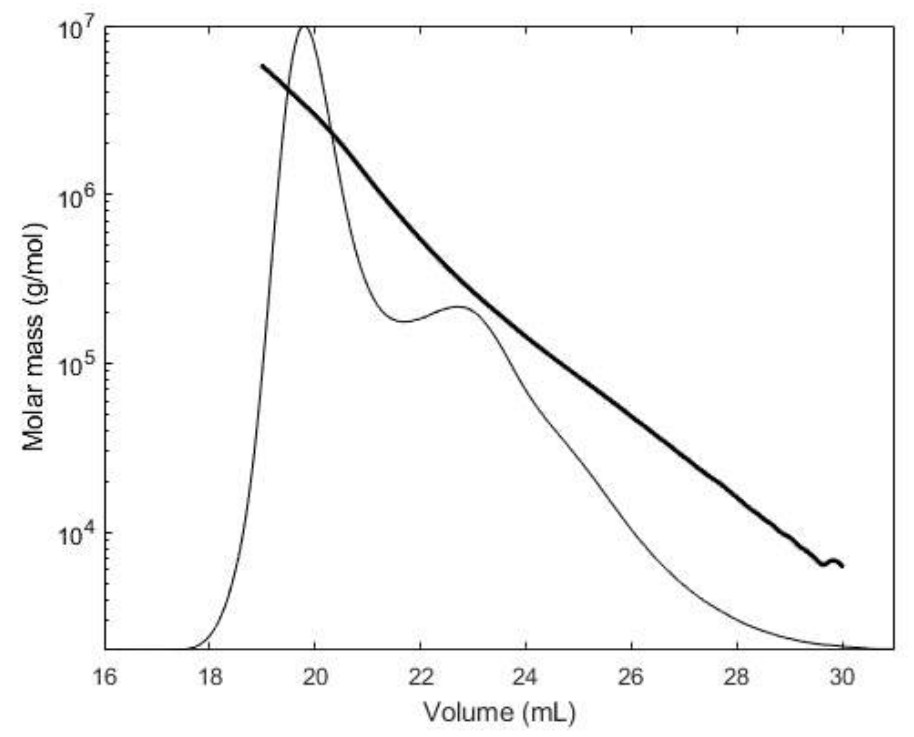

Figure 5.A.1: Molar weight distribution from SEC for dextran (D100).

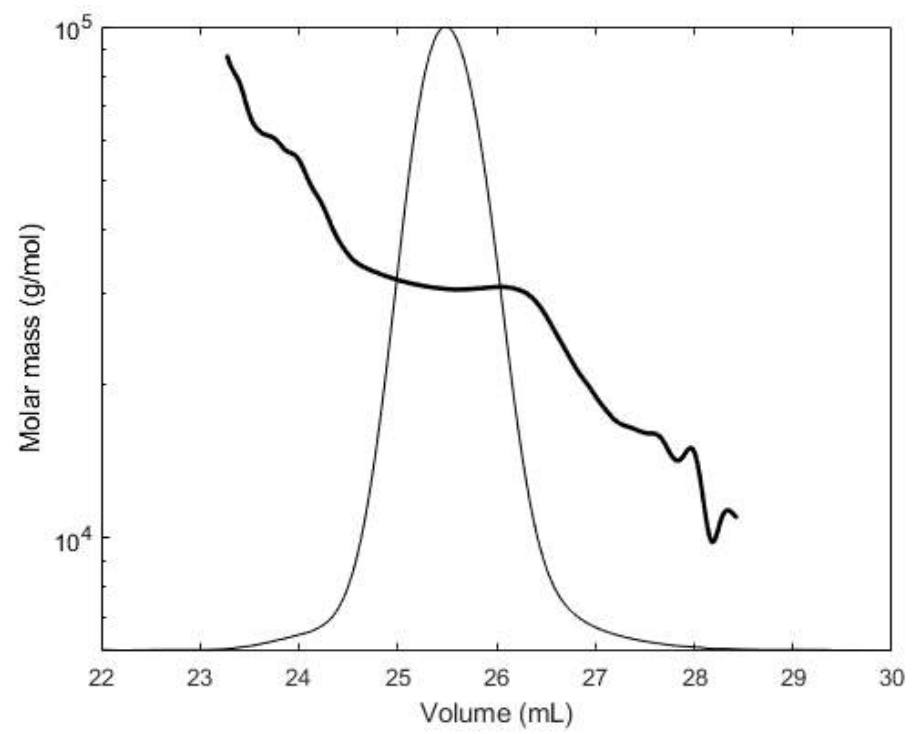

Figure 5.A.2: Molar weight distribution from SEC for polyethylene glycol (PEO35). 
General discussion 


\subsection{Introduction}

Practically relevant aqueous solutions consist generally of many components. Depending on the type of components in the solution, their interactions, and their concentration, these mixtures form a stable, homogeneous mixture or they are unstable and demix into two or more liquid phases. In the study of phase behavior of mixtures, these mixtures are often assumed to be binary mixtures of two monodisperse components. In this thesis we aimed to get a better understanding of the phase behavior of mixtures with more than two components in solution. Figure $\mathbf{6 . 1}$ gives an overview of the topics of each chapter.
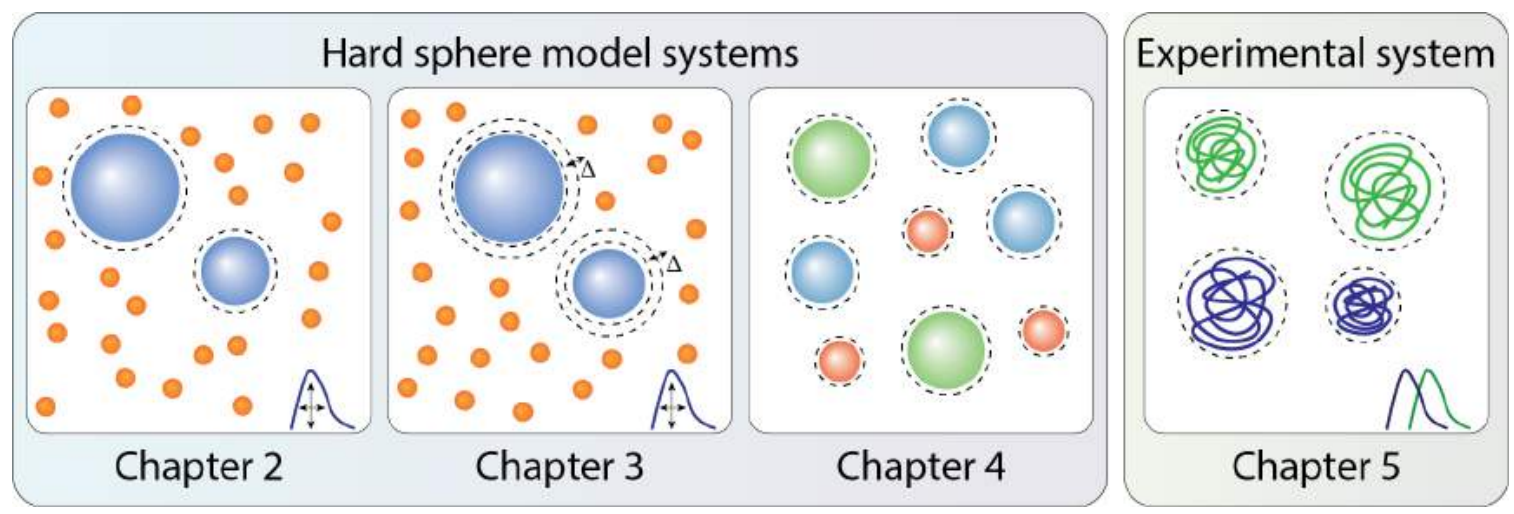

Figure 6.1: Schematic overview of thesis.

In Chapters 2 to 4 we studied the phase behavior of model systems of hard spheres. We started in Chapter 2 with the study of an asymmetric binary mixture of additive hard spheres (HS). This refers to spheres only interacting with their excluded volume, determined only by the sum of their radii. The larger component of the two was polydisperse. We took the polydispersity into account by sub-dividing this component into sub-components, and we varied the distribution and the number of sub-components. In the next chapter, Chapter 3, we continued building on this and incorporated so-called non-additivity into the system (NAHS). This implies an extra interaction contribution on top of the additive hard sphere interaction. Adding non-additivity to the subcomponents resulted into three phase regimes for some of the systems. In Chapter 4 we looked in more detail into the phase behavior of ternary hard spheres in solution. We studied how the addition of a third component alters the phase behavior of a binary mixture that phase separates. Some of the mixtures resulted in three phase systems. After having an understanding of the phase behavior of mixtures of hard spheres in solution we moved on to practical colloidal systems (Chapter 5) where we took the experimentally determined natural polydispersity of the colloids into account in predicting their phase behavior. We compared the theoretical phase diagram to already existing experimental results for the phase diagram. 


\subsection{Discussion and interpretation of results}

We first briefly summarize the findings of the separate chapters and combine the results with additional calculations in order to draw more general conclusions and compare these to the literature. We'll start by discussing the influence of size and size polydispersity on the phase behavior of additive hard spheres (Section 6.2.1). Next we will discuss the influence of non-additivity in mixtures of hard spheres (Section 6.2.2). In the third section (Section 6.2.3) we will discuss polydisperse colloidal systems. In the last section (Section 6.2.1) we will discus multi-phase systems in general.

\subsubsection{Effect of size polydispersity on the phase behavior of a binary mixture of hard spheres}

Particle size plays an important role in whether a binary mixture of hard spheres will phase separate and at what concentrations the mixture becomes unstable (Dijkstra et al., 1999). Following the methodology of Chapter 2, the critical point for mixtures can be determined. Figure 6.2 depicts the total volume of the particles at the critical point of a binary mixture depending on the size ratio $\left(q=\sigma_{B} / \sigma_{A}\right)$ between the two monodisperse components. The results are similar to the results of Ersch et al. (2016). The total particle volume of the mixture at the critical point changes drastically depending on the size ratio of the components. The plot is symmetric, indicating that it does not matter which one of the two is the smaller component and which one is the larger. The only thing that matters is that the size difference should be high enough. The lowest volume fraction of the critical point is at size ratios of $q=1 / 12.6$ and $q=12.6$. The critical point of a monodisperse binary mixture is the lowest concentration at which phase separation occurs. The particle size ratio therefore plays an important role in the position of the phase boundary of a mixture of two monodisperse components.

In Chapter 2 we studied the phase behavior of a polydisperse asymmetric binary mixture of additive hard spheres with a size ratio of $q=10$. The larger component of the two was polydisperse, while the smaller component remained monodisperse. We modeled the polydispersity using sub-components and considered a number of different distributions. The number average particle size of the polydisperse mixture remained the same for all considered mixtures. The polydispersity $(P D)$ was defined as:

$$
P D=\frac{\sqrt{\sum\left(\sigma_{B_{i}}-\sigma_{B}\right)^{2} \times N_{B_{i}} / N_{B}}}{\sigma_{B}} \times 100
$$

Depending on the chosen distribution and the degree of polydispersity the phase diagram 


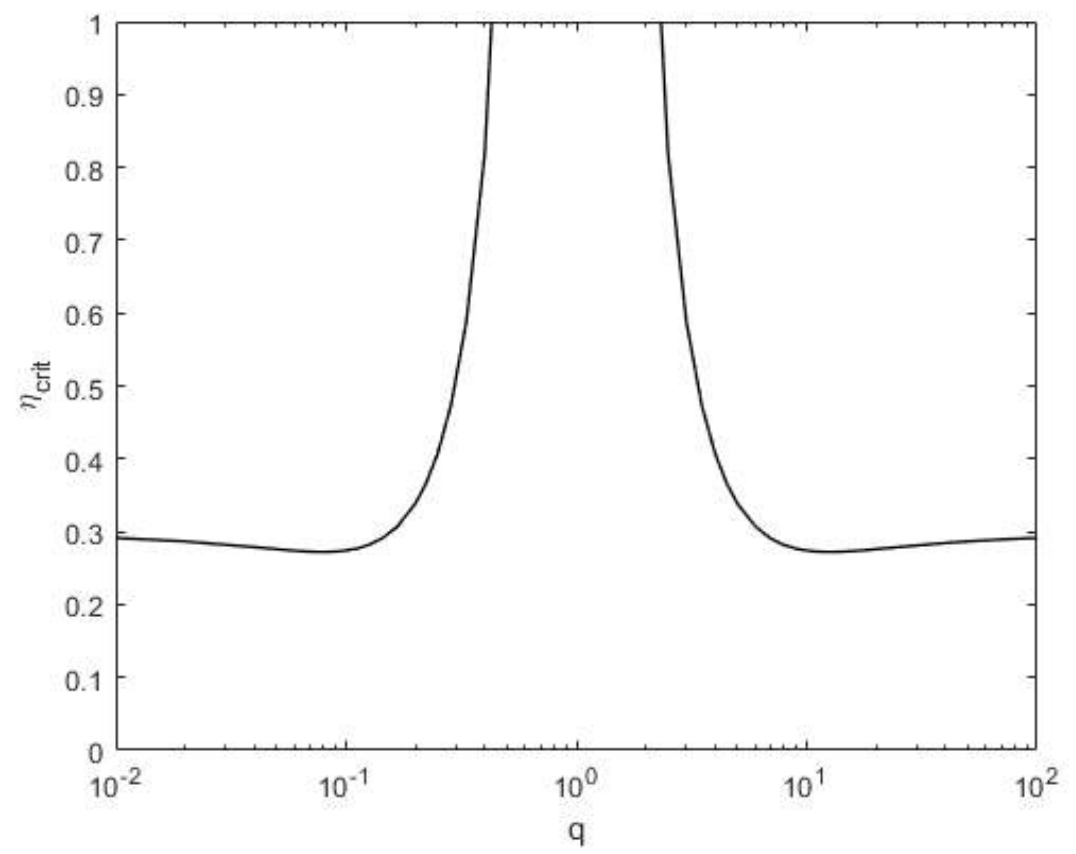

Figure 6.2: Total volume fraction at critical point $\left(\eta_{c r i t}=\eta_{c r i t_{A}}+\eta_{\text {crit }_{B}}\right)$ depending on size ratio $\left(q=\sigma_{B} / \sigma_{A}\right)$ in binary $\mathrm{HS}$ mixture. Left side when $B$ is the smallest of the two, right side when $B$ is the larger one of the two.

is altered. In general, when the size of the largest sub-component increased, e.g. due to a broad distribution (higher $P D$ ) or a skewed distribution, the critical point moved to higher volume fraction, with a higher packing fraction of the larger component $B$ and slightly lower packing fraction of component $A$. Next to that, the phase boundary shifts towards lower packing fractions of component $B$ at lower concentrations of $B$. The one-phase stable region therefore decreased.

In Chapter 2, we looked at a specific size difference between the large and the small component and considered only the large component as polydisperse. In order to get a more general understanding of the influence of size difference and polydispersity we calculated the critical point for mixtures at different degrees of polydispersity and different size ratios. In Figure 6.3 we varied the degrees of polydispersity (from 0.00 , i.e. monodisperse, to 20.00 with a step size of 4.00 ) of the polydisperse component $B$. The size distribution of $B$ follows a Gaussian distribution. Comparing the plot to Figure 6.2, we see that the plot is not symmetric anymore. When the polydisperse component is the smaller component of the two (left side of the figure), the polydispersity does not significantly influence the position of the critical point. When the polydisperse component is however the larger component of the two, the critical point shifts towards higher volume concentrations with increasing polydispersity.

The virial coefficients and also the excluded volume of the particles are directly related to 


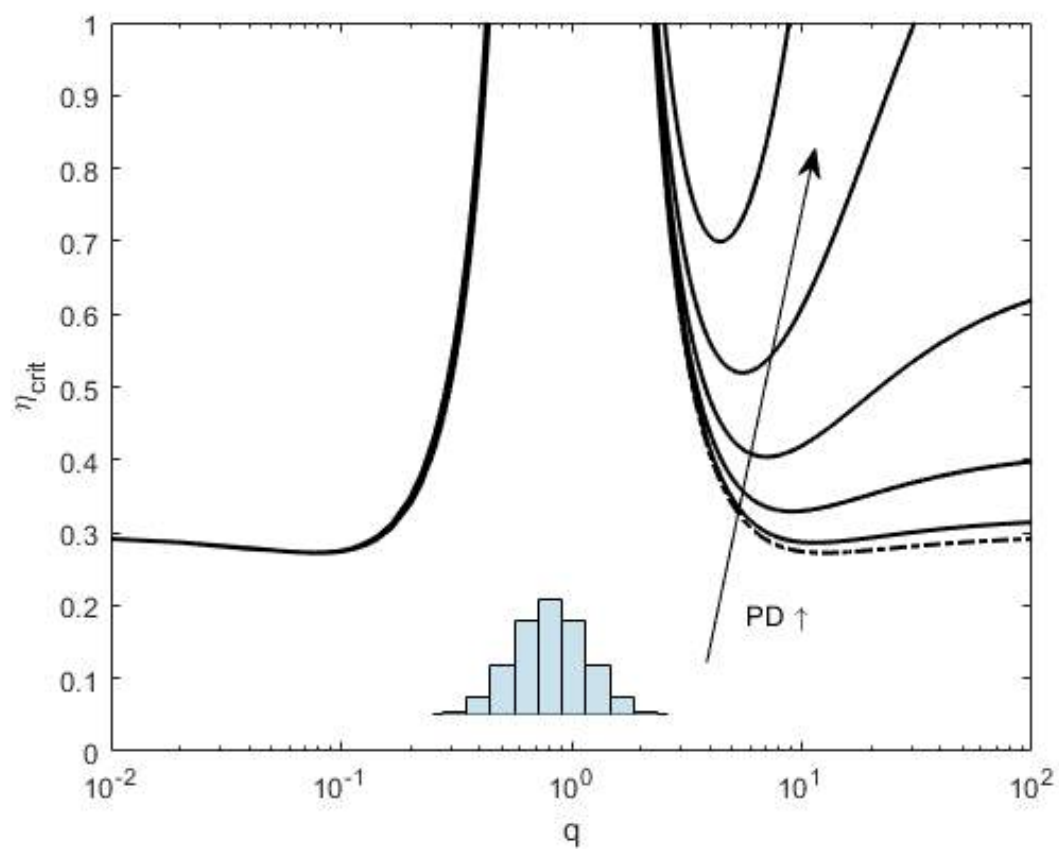

Figure 6.3: Total volume fraction at critical point $\left(\eta_{c r i t}=\eta_{c r i t}+\eta_{c r i t}\right)$ depending on size ratio ( $\left.q=\sigma_{B} / \sigma_{A}\right)$ and polydispersity of component $B$ binary mixture. The number of sub-components of the polydisperse component was nine and the distribution is given in the inset. The polydispersity, $P D$, was varied from 0.00 (monodisperse) to 20.00 with a stepsize of 4.00 . Arrows indicates increasing $P D$. Dashed line indicates $P D=0.00$. Left side when $B$ is the smallest of the two, right side when $B$ is the larger one of the two.

the volume of the particles, while the considered size difference are related to the diameter of these particles. When the size difference doubles, the excluded volume changes by a factor eight. Even smaller difference in diameter of larger particles therefore have a considerably high effect on the depletion interaction. Another factor influencing the phase diagram, is the choice of using the dimensionless parameter $\eta$ as a measure for concentration instead of for example a number based concentration. We kept the number average particle diameter the same, however their volume average will significantly change with the degree of polydispersity. The choice for the dimensionless parameter $\eta$ was made because this makes the phase diagram independent of the chosen radius, only the size ratio between particles matters.

We also found in Chapter 2 that the particle size distribution plays an important role in the position of the critical point. The distributions that contained larger sub-components shifted the critical point more. This can also be seen in Figure 6.4, where we compare different distributions of the polydisperse component $B$ with each the same number average size. When we consider a left skewed distribution (Figure 6.4a), a distribution with more small size sub-components, the critical point shifts to less high volume fractions at the same $P D$ and size ratio $q$ compared to the Gaussian distribution from Figure 6.3. When we consider a right skewed distribution (Figure $\mathbf{6 . 4 b}$ ) the critical point shifts to even 


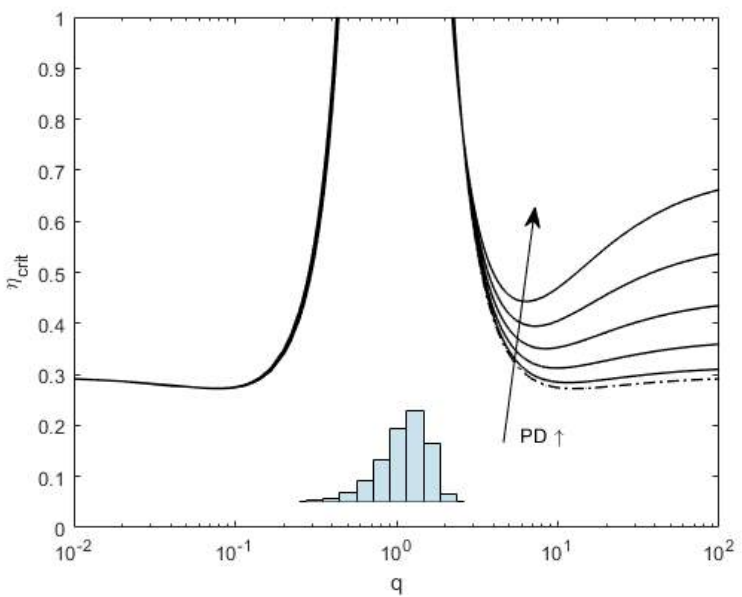

(a) Left skewed

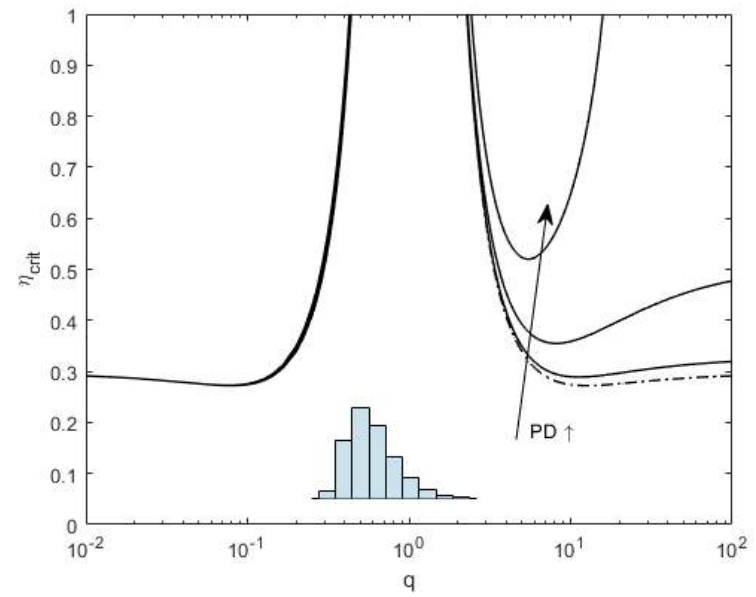

(b) Right skewed

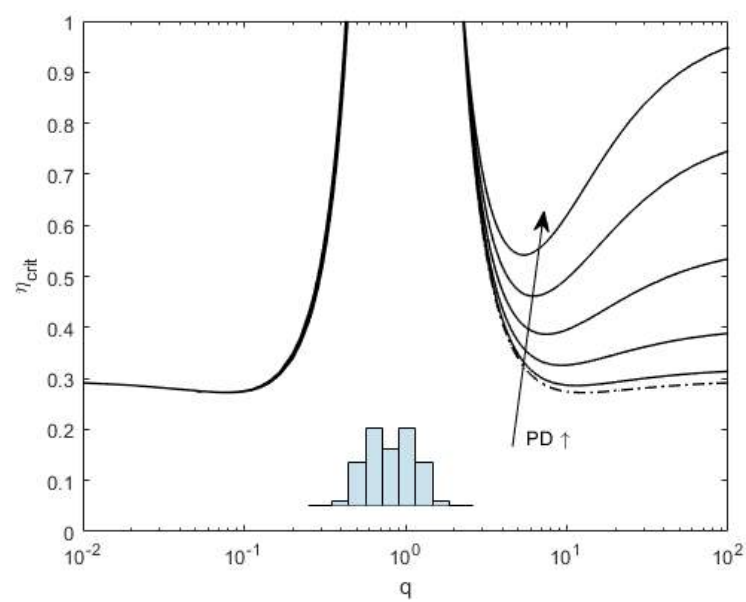

(c) Bimodal

Figure 6.4: Total volume fraction at critical point $\left(\eta_{c r i t}=\eta_{\text {crit }_{A}}+\eta_{\text {crit }_{B}}\right)$ depending on size ratio $\left(q=\sigma_{B} / \sigma_{A}\right)$ and polydispersity of component $B$ binary mixture. The number of sub-components of the polydisperse component was nine and the distribution is given in the inset. The polydispersity, $P D$, was varied from 0 (monodisperse) to 20 with a stepsize of 4 . Arrows indicates increasing $P D$. Dashed line indicates $P D=0$. Left side when $B$ is the smallest of the two, right side when $B$ is the larger one of the two.

more extreme high volume fractions. The critical point of the mixtures with the bimodal distribution (Figure 6.4c) has a lower effect on the shift in critical point of the mixture.

At relatively low polydispersity, the position of the critical point, is not significantly affected, independent of the chosen distribution. However, at larger polydispersity, the polydispersity as well as the particle distribution of the larger component have a considerable effect on the position of the critical point and therefore also on the phase behavior of the components.

For monodisperse mixtures the critical point can be used as a rule of thumb in predicting 
whether or not a mixture will demix. However, because the phase boundary shifts towards lower concentrations at the depleted side of the phase boundary of said polydisperse component (as we found in Chapter 2), it is still possible that a mixture demixes without finding a critical point. At extreme high polydispersity, the size ratio between the smallest and the largest particle of the polydisperse component might result in multi-phase behavior as was also reported earlier by Bellier-Castella et al. (2000). Additionally, the addition of a component to a polydisperse component might result in the demixing of the polydisperse component (see also Chapter $\mathbf{4}$, where the addition of the third component for some mixtures resulted in lower demixing concentrations).

Upon phase separation, particles will divide over the phases to minimize the Helmholtz free energy. One phase is enriched in component $A$, whilst the other is enriched in component $B$. Even though each phase is enriched in one component, the other component is still present, albeit at lower concentrations. In Chapter 2 we investigated the phase separation, in particular the fractionation and volume fraction of the phases $(\alpha)$, of a specific parent mixture for different distributions of component $B$. The size polydispersity of component $B$ lead to significant fractionation of this component in the child phases. The phase enriched in component $A$, which was the smaller component, contained also relatively more of the smaller particles of $B$ than the phase enriched in component $B$. This follows from the relatively increased size incompatibility between the smallest particle and the largest particle in the system. The polydispersity of component $B$ influences both the composition in each phase as well as the volume fraction of the phases. The volume fraction of the top phase, the phase enriched in component $A$ (the smaller and lighter component of the two), increased with the polydispersity of component $B$. The fractionation of the polydisperse component is not only dependent on the type of distribution, but also on the concentration of both components.

The fractionation and the volume fraction of each phase is also dependent on the parent concentration. Parent mixtures close to the critical point will show less fractionation compared to mixtures further away. Mixtures with a relatively low concentration of the polydisperse component $B$ will have significant fractionation. This fractionation is even more extreme when the concentrations are close to the phase boundary. For these mixtures, a large phase enriched in component $A$ will be formed and in this phase a small amount of the polydisperse component $B$ will be present. The size distribution of $B$ will be relatively similar to the distribution of the parent mixture. The other phase will be rather small and is enriched in the largest sub-components of component $B$. This results in a significantly different distribution and significantly larger average particle size. This is because the largest sub-components of component $B$ have the largest incompatibility with component $A$, which forms a driving factor in lowering the phase boundary and in depleting from the phase 

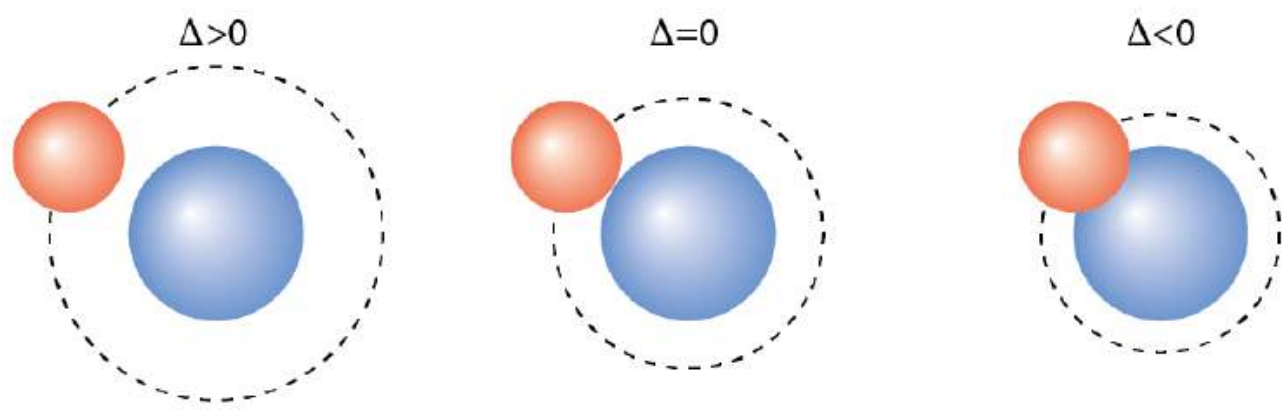

Figure 6.5: Visual representation of the depletion layer for (non-)additive hard spheres. When $\Delta>0$, the depletion layer becomes thicker compared to the additive model, while for $\Delta<0$, the depletion layer is thiner.

enriched in component $A$.

Until now we discussed the cases of hard sphere interactions. Now we extend to including non-additive hard sphere interaction.

\subsubsection{Effect of non-additivity}

Not only the size difference between particles plays a key role in phase separation, also their distance of closest approach is an important factor. For additive hard sphere mixtures $(\Delta=0)$, the distance of closest approach, i.e. the depletion layer is the sum of their radii $\sigma_{i j}=\left(\left(\sigma_{i}+\sigma_{j}\right) / 2\right)(1+\Delta)$. However, for non-additive mixtures, this distance can be larger or smaller (Figure 6.5) (Roth et al., 2001). A positive non-additivity $(\Delta>0)$ results in a thicker depletion layer and therefore more repulsion between the particles, leading to easier demixing (Hopkins and Schmidt, 2010). A negative non-additivity $(\Delta<0)$ results in a thinner depletion layer and therefore less repulsion between the particles, leading to more compatibility and more stability (Roth et al., 2001; Santos et al., 2010).

To get more insight into the influence on non-additivity on the phase behavior of monodisperse binary mixtures at different size ratios, we calculated the critical point for different mixtures (Figure 6.6). We varied the non-additivity parameter $\Delta$ from -0.1 to 0.5 with a step size of 0.05 . The plot is again symmetric, indicating that it does not matter which component is the larger one and which one is the smaller one. A negative non-additive parameter results in higher volume concentrations at the critical point, while a positive non-additive parameter decreases the particle volume at the critical point significantly. At higher non-additive parameters, due to the increased inter-particle repulsion for particles of different species, it becomes possible for particles of the same or similar size to phase separate, and at the highest $\Delta$ 's it is even easier for same size particles to demix compared to particles with very different sizes. 


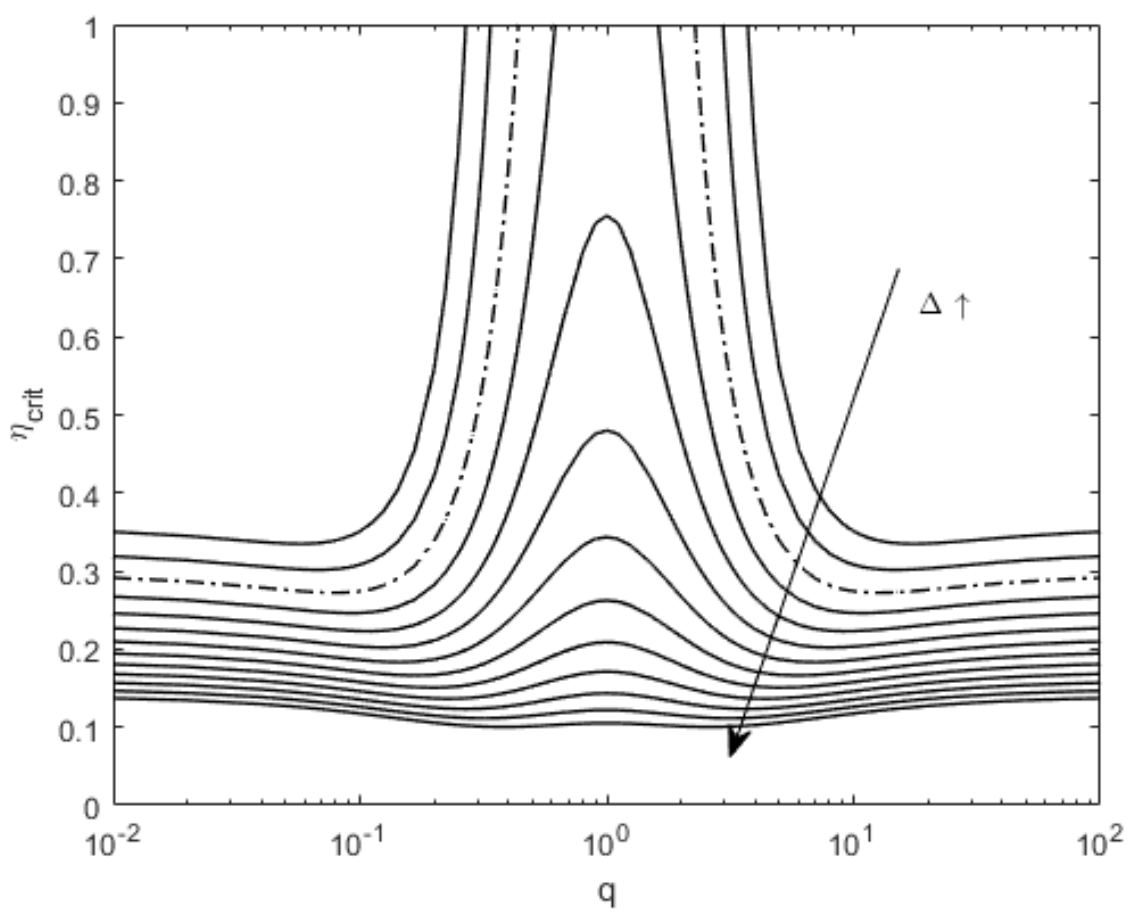

Figure 6.6: Total volume fraction at critical point $\left(\eta_{\text {crit }}=\eta_{\text {crit }_{B}}+\eta_{\text {crit }_{A}}\right)$ depending on size ratio $\left(q=\sigma_{B} / \sigma_{A}\right)$ and non-additivity parameter $\Delta$ in NAHS monodisperse binary mixture. $\Delta$ from -0.1 to 0.5 with a stepsize of 0.05 . Arrows indicates increasing $\Delta$. Dashed line indicates $\Delta=0$. Left side when $B$ is the smallest of the two, right side when $B$ is the larger one of the two.

In Chapter 3 we combined non-additivity with polydispersity. First we introduced nonadditivity between the monodisperse small component $A$ and the larger $(q=10)$ polydisperse component $B$. For a negative non-additivity, the critical point and phase boundary moved to higher volume concentration, while a positive non-additivity moved the critical point and phase boundary towards lower volume concentrations, just as for monodisperse mixtures (Hopkins and Schmidt, 2010). With higher non-additivity, the difference in critical point and phase boundary between the polydisperse mixture and the monodisperse mixture decreased. Just as for the additive mixtures, the critical point of these polydisperse mixtures moved to higher concentrations of the larger polydisperse component and slightly lower concentrations of the smaller component. This difference between the monodisperse reference mixture and the polydisperse system increased with polydispersity and with negative non-additivity.

Next we introduced a moderate $(-0.1 \leq \Delta \leq 0.1)$ non-additivity between the polydisperse particles of component $B$, while keeping the interaction between $A$ and $B$ additive. In order to limit the amount of interactions, we considered two sub-components of $B$, in a variety of distributions with the same number average size as before, varying the degree of polydispersity $P D$ (the size ratio between the particles) and the the skeweness of the distribution (the relative amount of each sub-component). 
Depending on the non-additivity, the polydispersity and the chosen distribution, the critical point shifts to higher or lower concentrations. Contrary to previous mixtures, mixtures with a negative non-additivity (increased compatibility between the sub-components of $B$ ) had a lower concentration at the critical point, while the critical point for mixtures with a positive non-additivity had higher concentrations at the critical point. For some of the mixtures, the critical point shifted to concentrations $\eta>0.5$. For mixtures with negative non-additivity, the phase enriched in component $B$ has a higher total particle volume concentration compared to completely additive mixtures. Due to the increased compatibility between the larger and smaller sub-components, it becomes easier for these components to be packed closely together. The volume fraction $(\alpha)$ was larger with decreasing $\Delta$ and less fractionation occurred. Mixtures with a positive non-additivity had a decreased compatibility between the small and large sub-components of $B$. They demixed at slightly higher concentrations compared to the additive mixtures. At higher concentrations, the mixtures demix into a three-phase system. At low concentrations of $B$ and high polydispersity, the position of the phase boundary became independent of the non-additivity. However at higher concentrations of $B$, increased polydispersity, and more positive non-additive interaction, the binodal started to bend towards the axis. With higher polydispersity, the shape of the three-phase boundary became independent of the non-additivity.

Parent mixtures demix into two or more phases, each phase enriched in one of the components. In Chapter 3 we also looked into the composition of the formed phases. For these mixtures, the smallest component $A$ preferentially demixed into the top phase, the phase with the largest volume. The volume fraction of both phases was dependent on the non-additivity of the larger polydisperse component $B$. Both positive as well as negative non-additivity increased the volume of the top phase. The non-additivity for which the top phase was smallest was different depending on both the PD as well as the distribution of $B$. The fractionation of the sub-components of $B$ is dependent on the non-additivity. When the sub-components are more compatible with each other, component $B$ has less fractionation, while on the other hand the sub-components are less compatible with each other and more fractionation occurs, even leading to additional phase separation at higher non-additivity. For mixtures that demixed into three phases, the bottom phase demixes into an additional middle phase enriched in the smaller sub-component of $B$.

In Chapter 4 we looked into the influence of a third component $C$ on a binary mixture $A B$ that demixes. We kept the interaction between $A$ and $B$ and the size of $C$ constant, while varying the non-additive interaction between $A-C$ and $B-C$. When $C$ had a negative non-additivity with both components, $C$ was more compatible with $A$ and $B$. This increased compatibility caused the phase-boundary between $A$ and $B$ to be independent of the concentration of $C$. Upon demixing, component $C$ is present in both phases in 
an amount similar to the parent phase. When the non-additive parameter is increased for the interaction between $C$ and one of the components, component $C$ becomes less compatible with this component. This causes the phase boundary to bend. Increasing the concentration of $C$ decreases the concentration of $A$ and $B$ required for demixing. Upon demixing into two phases, component $C$ preferentially goes to the phase enriched in the component, which component $C$ is most compatible with, increasing the volume of this phase. When the interaction between $C$ and both components is increased, component $C$ becomes less compatible with both $A$ and $B$. At high enough incompatibility amongst all three components, three-phase demixing becomes possible. In this case each of the three phases is enriched in one of the three components.

In general, depending on the incompatibility between all three components and the concentrations of the parent mixture, the composition of the demixed phase and the volume of each phase will be different. Mixtures with less compatibility among some of the components tend to demix at slightly lower concentration, compared to mixtures with high compatibility. Components that are relatively more compatible with each other tend to demix into the same phase increasing the volume of this phase. The addition of a third component that is incompatible with one or both components of a dilute binary mixture that can demix into two phases can cause the mixture to become unstable and demix. This was also shown experimentally by Johansson and Walter (1999) for the system polyethylene glycol and dextran, where a third polymer added to a dilute one phase mixture of polyethylene glycol and dextran caused the system to demix into two phases. At low concentrations, this third polymer distributes between the two phases (in general in non-equal amounts).

\subsubsection{Polydisperse colloidal systems}

In Chapter $\mathbf{5}$ we predicted the phase behavior of two polydisperse colloids, dextran and polyethylene glycol (PEO), based on their previously experimentally measured size distribution, osmotic virial coefficient and critical point. Compared to hard sphere model systems we studied before, the size difference between dextran and PEO was rather small and their size distribution even showed a considerable overlap. We compared our theoretical predictions to the experimental work from Dewi et al. (2020). The best fit to the experimental data was when dextran was polydisperse and PEO was monodisperse.

Polydispersity causes the phase boundary to bend and broaden towards lower concentrations, especially at low concentration of the most polydisperse component (dextran). The predicted shape of the phase boundary turns into a W-shape instead of a U-shape. This was also experimentally shown by Larsson and Mattiasson (1988). They have shown that this shift in the phase boundary to lower concentrations increases with increasing polydispersity 
at low concentrations of said polydisperse component (Larsson and Mattiasson, 1992). Because of this broadening, the one-phase stable region for the mixture decreases (Kang and Sandler, 1988; Döbert et al., 1995).

At concentrations where both components are present in a significant amount, e.g. near the critical point and at higher concentrations, the spinodal follows the spinodal of the monodisperse fitting. At these concentrations the bulk interactions between all subcomponents of PEO and dextran govern the interaction and the resulting instability of the mixture. In the meta-stable region, the region between the spinodal and the binodal, the demixing is determined by the components that are most incompatible, e.g. the largest sub-component of dextran and PEO.

Upon demixing, a parent mixture demixes into two phases, each enriched in either dextran or PEO. However, the size distribution of PEO and dextran in the demixed system can differ significantly from the parent distribution. The change in the distribution is not only dependent on the interactions between the components, but also on the chosen parent concentration. In general, the phase enriched in dextran will contain more of the larger dextran sub-components and relatively more of the smaller PEO sub-components. The size distributions in the demixed phases resembles the one from the parent mixture more along the dilution line through the critical point. Further away from the critical point, e.g. samples with high PEO concentrations, the fractionation is significant as the phase enriched in dextran contains relatively more and more larger dextran molecules when decreasing the concentration. Experimental work by Edelman et al. (2003), Zhao et al. (2016) and Gaube et al. (1993) are in line with these predictions.

\subsubsection{Multi-phase systems}

When the incompatibility among multiple components is high enough, demixing into more than two phases becomes possible. In Chapter $\mathbf{3}$ we saw that the polydispersity or small impurities of a mixture that is stable by itself could cause demixing into more than two phases when an incompatible component was added. The position of the three phase boundary was dependent on the polydispersity, the distribution of the components and the non-additivity between the polydisperse sub-components. In Chapter 4 we looked in detail into the interaction of three components in a system. We saw that the shape and the position of the three phase boundary was dependent on the incompatibility among all three components.

The lowest concentration of the three-phase boundary is on the spinodal, the plait point line and also the binodal. This is a special plait point, where all three phases become indistinguishable. Small deviations in one of the concentrations of the three components 


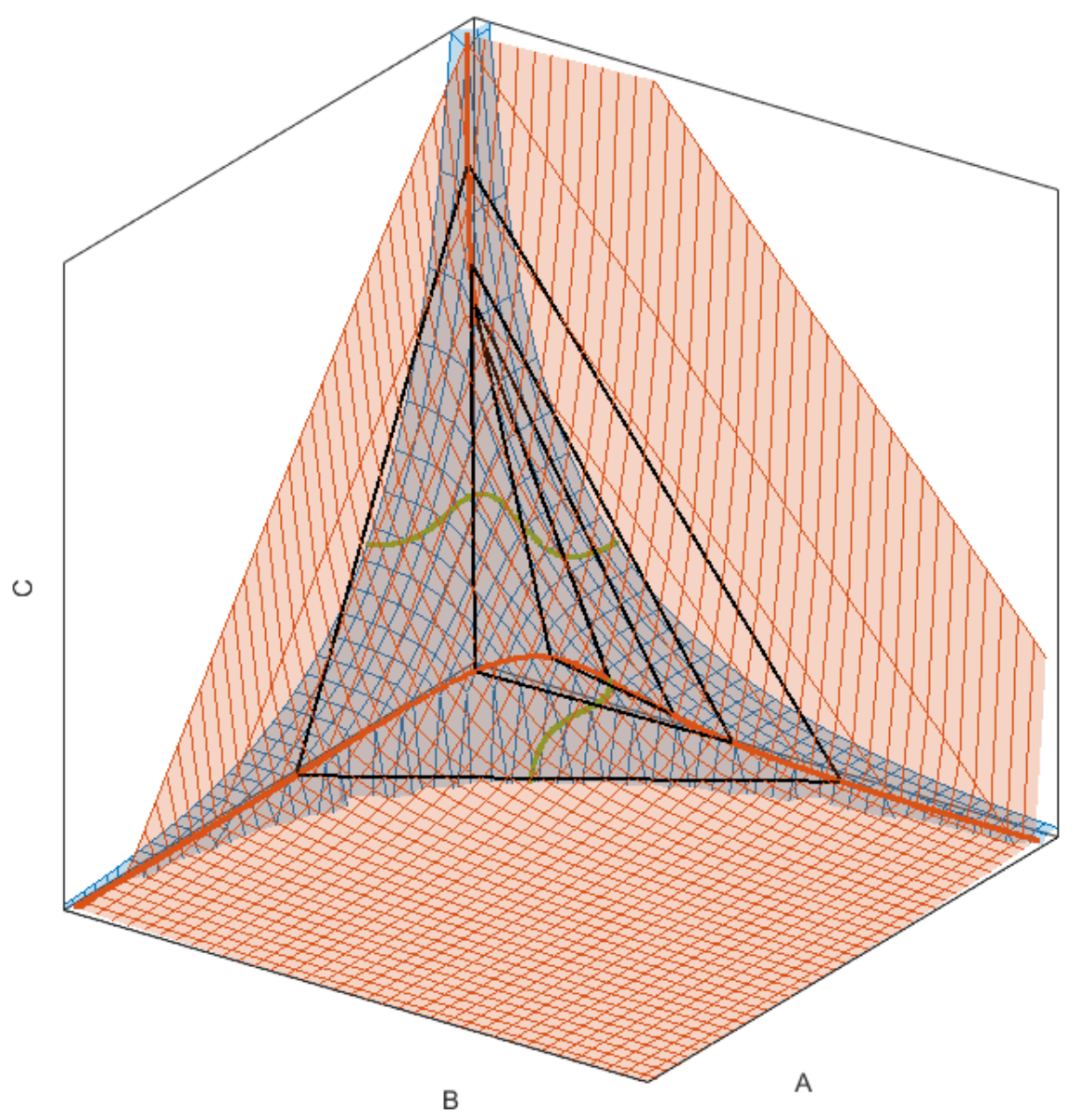

Figure 6.7: Theoretical three phase system. The three phase boundary lays on the two phase boundary. Plait points are indicated in green. The two phase boundary is indicated in blue. The three phase boundary is indicated in red. Some tie-lines for mixtures that result in three equal phases are indicated in black.

will result in the formation of one homogeneous phase, two phases (depending on the perturbation, these phases are enriched in different components), or three phases see also Figure 6.7. The transition from two phases to three phases is along a tie-line of the two phase boundary. Increasing the concentration slightly, results in demixing of one the phases and forming the third phase. Which phase will become unstable is dependent on the relative incompatibility between the phases. Depending on the position of the critical point of the sub-mixtures, the three-phase boundary surface will rotate. In Figure 6.7, the sub-mixture $A-B$ had the highest critical point concentration of the three.

Experimental three phase systems of, for example, the colloids dextran, ficoll, and 


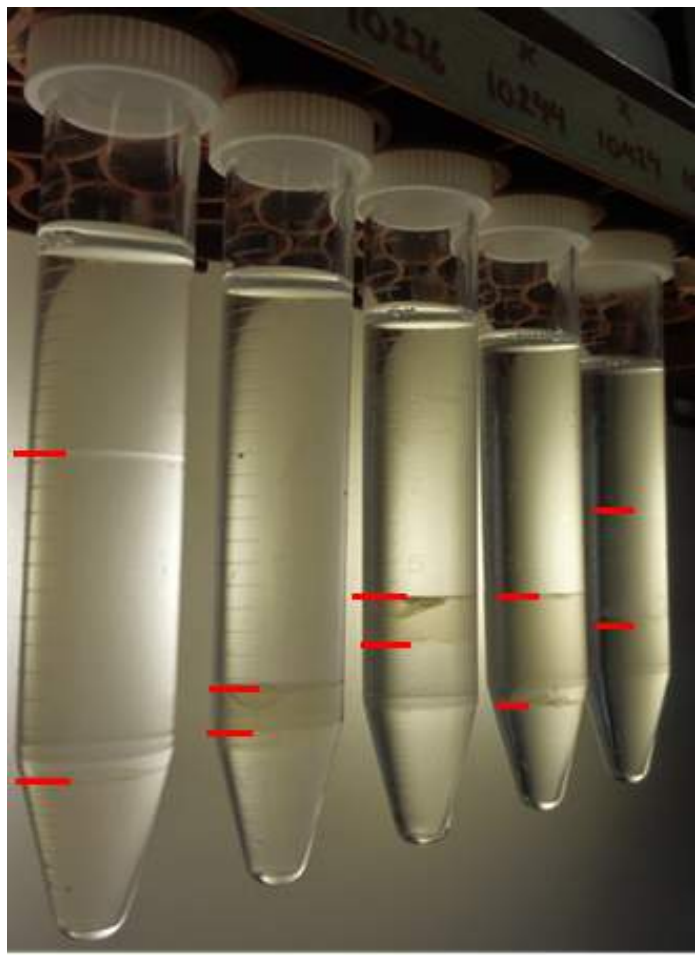

Figure 6.8: Three phase system of gelatin, dextran, and polyethylene glycol in a variety of concentrations, the red mark indicates a phase boundary.

polyethylene-glycol (Hartman et al., 1974) and dextran, polyethylene-glycol, and gelatin (unpublished work and Figure 6.8) show similar phase behavior. Depending on the concentrations of each component, the system will form one phase or demix into two or three phases, each phase enriched in one of the components. Other examples of multi phase systems are fore example for the system of the colloids dextran and polyethylene-glycol with the surfactant Triton X-100 (Ruan et al., 2006). Mixtures with an even higher number of components (both colloids and surfactants) and a corresponding higher number of phases in equilibrium were studied by Mace et al. (2012). They report systems of up to six phases, each enriched in one of the polymers and depleted in the others. The methodology developed in this thesis may be further tested for these multi-component mixtures

\subsection{Concluding remarks}

In this thesis we used the virial approach to predict the phase behavior of polydisperse and multi-component mixtures in solution. The theory allowed for predicting the critical point, the phase boundary and the instability region (spinodal). Next to that the theory allows for predicting the composition of demixed phases and the volume of each phase. Our approach yields results in line with previous theoretical and experimental work on polydisperse mixtures. 
The virial theory allows for direct experimental testing using virial coefficients obtained from membrane osmometry and can be a huge advantage in predicting the existence of multi-phase behavior. 


\subsection{Bibliography}

L. Bellier-Castella, H. Xu, and M. Baus. Phase diagrams of polydisperse van der Waals fluids. Journal of Chemical Physics, 113(18):8337-8347, 2000. ISSN 00219606. doi: 10.1063/1.1316007.

B. P. C. Dewi, E. van der Linden, A. Bot, and P. Venema. Second order virial coefficients from phase diagrams. Food Hydrocolloids, 101:105546, 2020. ISSN 0268005X. doi: 10.1016/j.foodhyd.2019.105546.

M. Dijkstra, R. van Roij, and R. Evans. Phase diagram of highly asymmetric binary hardsphere mixtures. Physical Review E - Statistical Physics, Plasmas, Fluids, and Related Interdisciplinary Topics, 59(5):5744-5771, 1999. ISSN 1063651X. doi: 10.1103/PhysRevE.59.5744.

F. Döbert, A. Pfennig, and M. Stumpf. Derivation of the Consistent Osmotic Virial Equation and its Application to Aqueous Poly(ethylene glycol)-Dextran Two-Phase Systems. Macromolecules, 28(23):7860-7868, 1995. ISSN 15205835. doi: 10.1021/ma00127a037.

M. W. Edelman, E. Van Der Linden, and R. H. Tromp. Phase separation of aqueous mixtures of poly(ethylene oxide) and dextran. Macromolecules, 36(20):7783-7790, 2003. ISSN 00249297. doi: 10.1021/ma0341622.

C. Ersch, E. van der Linden, A. Martin, and P. Venema. Interactions in protein mixtures. Part II: A virial approach to predict phase behavior. Food Hydrocolloids, 52:991-1002, 2016. doi: 10.1016/j.foodhyd.2015.07.021.

J. Gaube, R. Höchemer, B. Keil, and A. Pfennig. Polydispersity Effects in the System Poly(ethylene glycol) + Dextran + Water. Journal of Chemical and Engineering Data, 38(2):207-210, 1993. ISSN 15205134. doi: 10.1021/je00010a005.

A. Hartman, G. Johansson, and P. Albertsson. Partition of Proteins in a Three-Phase System. European Journal of Biochemistry, 46(1):75-81, 1974. ISSN 14321033. doi: 10.1111/j.1432-1033.1974.tb03598.x.

P. Hopkins and M. Schmidt. Binary non-additive hard sphere mixtures: Fluid demixing, asymptotic decay of correlations and free fluid interfaces. Journal of Physics Condensed Matter, 22(32), 2010. ISSN 09538984. doi: 10.1088/0953-8984/22/32/325108.

G. Johansson and $\mathrm{H}$. Walter. Partitioning and concentrating biomaterials in aqueous phase systems. International Review of Cytology, 192(1896):33-60, 1999. ISSN 00747696. doi: 10.1016/s0074-7696(08)60521-5. 
C. H. Kang and S. I. Sandler. Effects of polydispersivity on the phase behavior of the aqueous two-phase polymer systems. Macromolecules, 21(10):3088-3095, 1988. ISSN 0024-9297.

M. Larsson and B. Mattiasson. Characterization of aqueous two-phase systems based on polydisperse phase forming polymers: Enzymatic hydrolysis of starch in a PEG-starch aqueous two-phase system. Biotechnology and Bioengineering, 31(9):979-983, 1988. ISSN 10970290. doi: 10.1002/bit.260310910.

M. Larsson and B. Mattiasson. Molecular Weight Distribution Analysis of Water-Soluble Polymers Using Aqueous Two-Phase Systems. Annals of the New York Academy of Sciences, 672(1 Enzyme Engine):649-656, nov 1992. ISSN 0077-8923. doi: 10.1111/j.17496632.1992.tb35685.x.

C. R. Mace, O. Akbulut, A. A. Kumar, N. D. Shapiro, R. Derda, M. R. Patton, and G. M. Whitesides. Aqueous Multiphase Systems of Polymers and Surfactants Provide Self-Assembling Step-Gradients in Density. Journal of the American Chemical Society, 134(22):9094-9097, 2012. ISSN 0002-7863. doi: 10.1021/ja303183z.

R. Roth, R. Evans, and A. A. Louis. Theory of asymmetric nonadditive binary hard-sphere mixtures. Physical Review E - Statistical Physics, Plasmas, Fluids, and Related Interdisciplinary Topics, 64(5):13, 2001. ISSN 1063651X. doi: 10.1103/PhysRevE.64.051202.

K. Ruan, B. H. Wang, J. X. Xiao, and J. N. Tang. Interfacial tension of aqueous threephase systems formed by Triton X-100/PEG/Dextran. Journal of Dispersion Science and Technology, 27(7):927-930, 2006. ISSN 01932691. doi: 10.1080/01932690600766223.

A. Santos, M. López De Haro, and S. B. Yuste. Virial coefficients, thermodynamic properties, and fluid-fluid transition of nonadditive hard-sphere mixtures. Journal of Chemical Physics, 132(20), 2010. ISSN 00219606. doi: 10.1063/1.3429600.

Z. Zhao, Q. Li, X. Ji, R. Dimova, R. Lipowsky, and Y. Liu. Molar mass fractionation in aqueous two-phase polymer solutions of dextran and poly(ethylene glycol). Journal of Chromatography A, 1452:107-115, 2016. ISSN 18733778. doi: 10.1016/j.chroma.2016.04.075. 
Summary 
In this thesis we aimed to get a better understanding of the phase behavior of polydisperse and multi-component mixtures in solution. We approached this in a systematic way, starting with model systems of hard spheres and ending with a system of polydisperse macromolecules.

In Chapter 1 we presented a short overview of the available literature on polydisperse en multi-component mixtures that show segregative phase separation.

In Chapter 2 we reviewed the theory of interactions in systems of a solute component in a solvent based on the second virial coefficient. The theory was expanded to allow for multiple distinguishable types of solute components. This chapter describes the general equations used in the thesis that define the phase boundary, stability of the mixture, and critical point. Next to the theory, the chapter also dealt with the effect of polydispersity on the phase behavior of a binary mixture of additive hard spheres. This chapter showed that the largest species in the polydisperse component had the largest influence on the changes in the phase diagram.

In Chapter 3 we deal with the effect of non-additivity on the phase behavior of a binary mixture of hard spheres with slight polydispersity or impurities. Non-additivity between the two polydisperse and monodisperse component shifts the phase boundary to higher (negative non-additivity) or lower (positive non-additivity) concentrations. Negative non-additivity within a polydisperse component decreased overall compatibility and lowered the phase boundary. Positive non-additivity within a polydisperse component pushed the two-phase boundary to slightly higher concentrations and made three-phase demixing possible.

In Chapter $\mathbf{4}$ we discussed the influence of a third component added to a binary mixture that is incompatible. When the third component is compatible with both binary components, the phase boundary is not altered and upon demixing, the third component is present in both phase in similar amounts as in the parent mixture. When the third component is incompatible with one of the other two components, the phase boundary bends towards the axis-plane. Upon demixing, the third component preferentially goes to the phase enriched in the component with highest relative compatibility. When the third component is incompatible with both, three-phase demixing becomes possible.

In Chapter 5 we applied the gained knowledge from the model systems to a binary mixture of the polydisperse polymers polyethylene glycol and dextran. The phase behavior of the macromolecules was theoretically predicted, taking their experimental osmometric second virial coefficients and size polydispersity into account, and compared to the previously published experimental phase diagram. Taking polydispersity into account allowed for a better prediction of the experimental phase boundary compared to assuming monodispersity.

In Chapter $\mathbf{6}$ we discussed our results from the different chapters and placed the current work in a wider context of the available scientific literature, and discuss future research on multi-component and polydisperse mixtures in solution. 
In conclusion, the virial approach we used has yielded results in line with previous theoretical and experimental work on polydisperse mixtures and mixtures of more than two components, and at the same time allows for direct experimental testing of the theoretical approach using virial coefficients directly obtained from membrane osmometry. 



\section{Acknowledgments}



Many people contributed in one way or another to the completion of this thesis. I would like to thank a few of you in particular.

Firstly, I would like to thank my supervisors Erik and Harald for your support, enthusiasm and guidance. Next to that I would like to thank my collogues at FPH and in particular Els and my office mates Claudine, Belinda, Arianne and Philipp, you made the work the office more enjoyable. Additionally, I would like to thank all the students who did their thesis project on my subject.

I'm also very grateful for the support I got from friends. I especially want to thank my friends in Belgium who always believed in me, the Soda Stereo pub quiz team with whom I spend many entertaining evenings and my bouldering buddies who gave me the necessary physical distractions from time to time.

Last, but not least I want to thank my mom for her never ending support. 

About the author 



\section{Biography}

Luka Sturtewagen was born in the spring of 1988 in Gent, Belgium. In 2006, Luka moved for a year to 能勢町, 大阪府,日本国 (Nose-chō, Osaka, Japan) for a cultural exchange program to learn Japanese.

After returning from Japan, they moved to Ghent to study Life Science Engineering at Ghent University. As part of their study, they spend six months at the Universität für Bodenkultur in Vienna and took a course at Polytech Lille. During their internship at Alpro, they investigated the influence of small peptides on the fermentation rate of soy-based yoghurt alternative. Their master thesis research was conducted at the Environmental Organic Chemistry and Technology department, in collaboration with Sodexo and Johnson \& Johnson. The research was focused on the resource use profile and nutritional value of a canteen meal. For their thesis research, Luka was bestowed with the Vandemoortele Healthy Foods thesis award.

In 2015, Luka moved to the Netherlands to start their PhD research at Wageningen University at the Laboratory of Physics and Physical Chemistry of Foods. This thesis was the result of their research. In 2020, Luka started a new position as a data engineer at LINKIT, Utrecht. 


\section{List of publications}

L. Sturtewagen, W. De Soete, J. Dewulf, C. Lachat, S. Lauryssen, B. Heirman, F. Rossi,

T. Schaubroeck (2016). Resource use profile and nutritional value assessment of a typical Belgian meal, catered or home cooked, with pork or Quorn ${ }^{\mathrm{TM}}$ as protein source. Journal of Cleaner Production,112 (1), 196-204.

L. Sturtewagen and E. van der Linden. Effect of polydispersity on the phase behavior of additive hard spheres in solution, part I. (submitted)

L. Sturtewagen and E. van der Linden. Effect of polydispersity on the phase behavior of non-additive hard spheres in solution, part II. (submitted)

L. Sturtewagen and E. van der Linden. Effect of third component on the phase behavior of a binary mixture of hard spheres in solution. (to be submitted)

L. Sturtewagen, B.P.C. Dewi, P. Venema and E. van der Linden. Improving predictions of phase behavior of binary mixtures by taking polydispersity into account. (to be submitted)

L. Sturtewagen, H. van Mil, M. Devezeaux de Lavergne, M. Stieger, E. van der Linden and T. Odijk. A quantitative Information Measure applied to texture perception attributes during mastication. (to be submitted)

L. Sturtewagen, H. van Mil and E. van der Linden. Complexity in sensory science. (to be submitted) 


\section{Overview of completed training activities}

\section{Discipline specific activities}

\begin{tabular}{|c|c|c|c|}
\hline 2016 & $\begin{array}{l}\text { NTU Winter School: Introduction to } \\
\text { Complexity Science }\end{array}$ & NTU & Singapore, SG \\
\hline 2016 & $7^{\text {th }}$ Sensory Perception and Food Prefence & VLAG & Wageningen, NL \\
\hline 2016 & $16^{\text {th }}$ Food Colloids Conference & WUR + VLAG & Wageningen, NL \\
\hline 2016 & $\begin{array}{l}4^{\text {th }} \text { International conference on Food Oral } \\
\text { Processing }\end{array}$ & $\mathrm{EPFL}+$ Nestle & Lausanne, CF \\
\hline 2016 & Conference on Complex Systems & CSS & Amsterdam, NL \\
\hline 2016 & $\begin{array}{l}\text { Symposium Sensory and liking - inspira- } \\
\text { tion for Application }\end{array}$ & TIFN & Groningen, NL \\
\hline 2020 & Physics@Veldhoven & NWO & Veldhoven, NL \\
\hline 2020 & $18^{\text {th }}$ Food Colloids Conference* & LU & Lund, SE \\
\hline
\end{tabular}

${ }^{*}$ Canceled due to the COVID19 pandemic 


\section{General courses and activities}

$\begin{array}{llll}2016 & \text { VLAG PhD week } & \text { VLAG } & \text { Soest, NL } \\ 2016 & \text { PhD Workshop Carousel } & \text { WGS } & \text { Wageningen, NL } \\ 2016 & \begin{array}{l}\text { Techniques for writing and presenting a } \\ \text { scientific paper }\end{array} & \text { WGS } & \text { Wageningen, NL } \\ 2016 & \text { Scientific Writing } & \text { In'to Languages } & \text { Wageningen, NL } \\ 2017 & \begin{array}{l}\text { Philosophy and Ethics of Food Science \& } \\ \text { Technology }\end{array} & \text { VLAG } & \text { Wageningen, NL } \\ 2017 & \begin{array}{l}\text { Interpersonal Communication for PhD } \\ \text { candidates }\end{array} & \text { WGS } & \text { Wageningen, NL } \\ 2017 & \text { Adobe InDesign Essential Training } & \text { WUR Library } & \text { Wageningen, NL } \\ 2018 & \text { Presenting with impact } & \text { WGS } & \text { Wageningen, NL } \\ 2018 & \text { Inclusiveness for better study results } & \text { 4TU } & \text { Wageningen, NL }\end{array}$

\section{Optional courses and activities}

$\begin{array}{lllr}2015 & \text { National PhD day Delft } & \text { TUDelft } & \text { Delft, NL } \\ 2015 & \text { Preparation of research proposal } & \text { Research group } & \text { Wageningen, NL } \\ 2015-2019 & \text { Science meetings } & \text { Research group } & \text { Wageningen, NL } \\ 2018 & \text { PhD Trip to Singapore and Indonesia } & \text { Research group } & \text { SG + ID } \\ 2018 & \text { Gender and Diversity in consumer culture } & \text { WUR } & \text { Wageningen, NL }\end{array}$



Cover design by Luka Sturtewagen

Printed on $100 \%$ recycled paper by GVO Drukkers \& vormgevers B.V. 